\title{
Physiological and molecular aspects of ovarian follicular developmental competence in sows
}

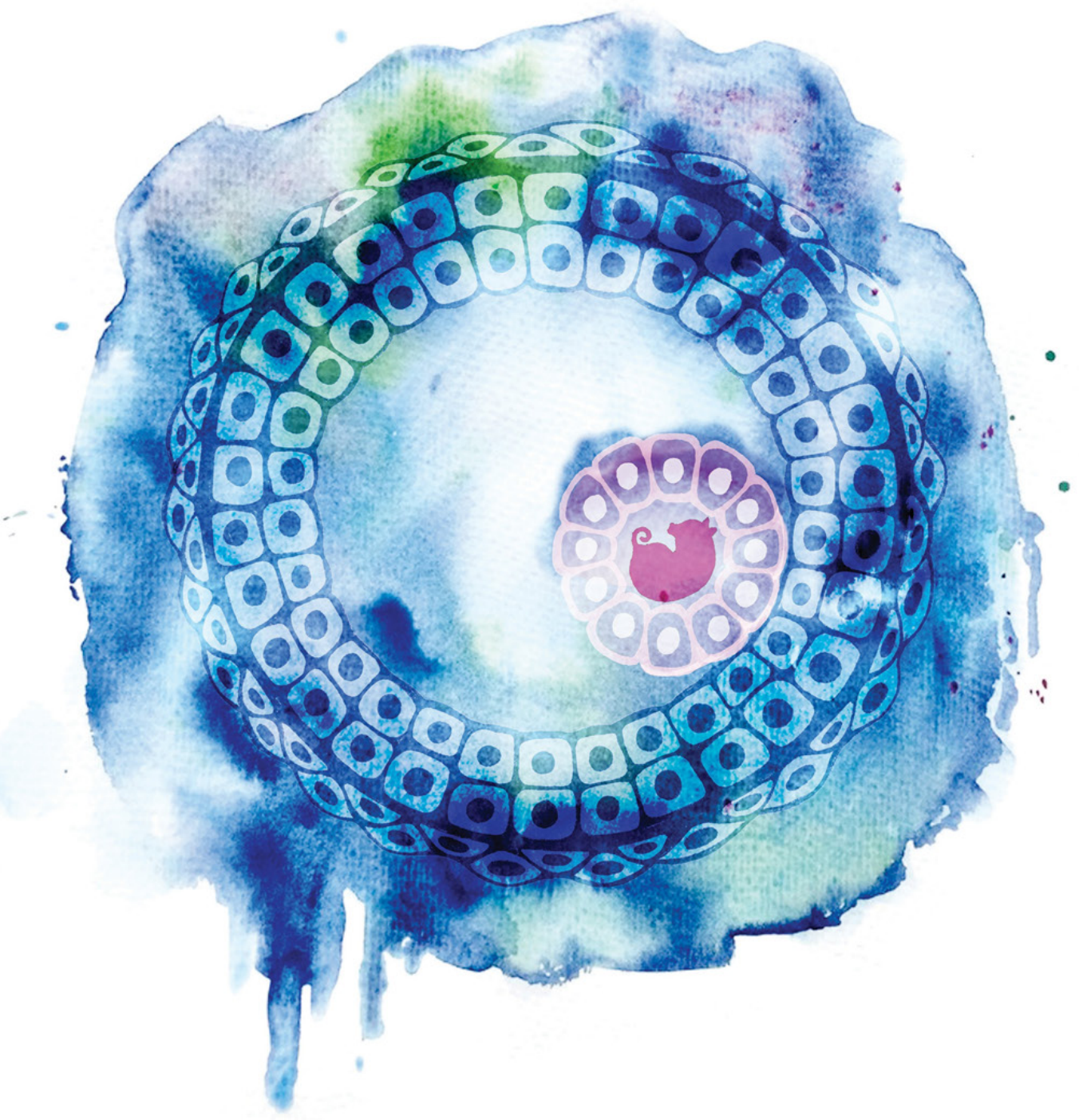

Natasja G. J. Costermans 


\section{Propositions}

1. Impairments in follicular development during lactation and around weaning contribute to low piglet birth weights and variation in piglet birth weight.

(this thesis)

2. Lactational feed restriction impairs follicular steroid and growth factor production which reduces oocyte developmental competence.

(this thesis)

3. Sows are a suitable model to study reproductive physiology in humans.

4. Mechanistic studies are essential to solve current challenges in animal production.

5. An individual's walking speed is a determinant of cognitive function.

6. The paternal contribution to embryo development is severely underestimated.

7. Many animal experiments can be prevented by actively involving scientists outside the project group.

8. Journals should stimulate co-peer review by junior researchers.

Propositions belonging to the thesis, entitled

Physiological and molecular aspects of ovarian follicular developmental competence in sows

Natasja G. J. Costermans

Wageningen, 25 March 2020 
Physiological and molecular aspects of ovarian follicular developmental competence in sows

Natasja G. J. Costermans 


\section{Thesis committee}

\section{Promotors}

Prof. Dr Jaap Keijer

Professor of Human and Animal Physiology

Wageningen University \& Research

Prof. Dr Bas Kemp

Professor of Adaptation Physiology

Wageningen University \& Research

\section{Co-promotors}

Dr Nicoline M. Soede

Associate professor, Adaptation Physiology Group

Wageningen University \& Research

Dr Katja J. Teerds

Associate professor, Human and Animal Physiology

Wageningen University \& Research

\section{Other members}

Prof. Dr Walter J. J. Gerrits, Wageningen University \& Research

Prof. Dr Ans M. M. van Pelt, Amsterdam UMC

Prof. Dr Olli Peltoniemi, University of Helsinki, Finland

Prof. Dr Ann van Soom, Ghent University, Belgium

This research was conducted under the auspices of the Graduate School Wageningen Institute of Animal Sciences 


\section{Physiological and molecular aspects of ovarian follicular developmental competence in sows}

Natasja G. J. Costermans

Thesis

submitted in fulfilment of the requirements for the degree of doctor at Wageningen University

by the authority of the Rector Magnificus,

Prof. Dr A. P. J. Mol,

in the presence of the

Thesis Committee appointed by the Academic Board

to be defended in public

on Wednesday 25 March 2020

at 1.30 p.m. in the Aula 
Natasja G. J. Costermans

Physiological and molecular aspects of ovarian follicular developmental competence in sows, 210 pages.

PhD thesis, Wageningen University, Wageningen, the Netherlands (2019)

With references, with summary in English

ISBN: 978-94-6395-161-6

DOI: https://doi.org/10.18174/502793 


\section{Table of contents}

Chapter 1 General Introduction

Chapter 2 Follicular development of sows at weaning in relation to estimated breeding value for within-litter variation in piglet birth weight

Chapter $\mathbf{3}$ In ovaries with high or low variation in follicle size, granulosa cells of antral follicles exhibit distinct size-related processes

Chapter 4 Follicular fluid steroid profile in sows: relation to follicle size and oocyte quality

Chapter 5 Steroid profile of porcine follicular fluid and blood serum: relation with follicular development

Chapter 6 Consequences of negative energy balance on follicular development and oocyte quality in primiparous sows

Chapter $\mathbf{7}$ Influence of the metabolic state during lactation on milk production in modern sows

Chapter 8 General Discussion

Appendices Summary of main findings

Acknowledgements

About the author

Curriculum vitae 



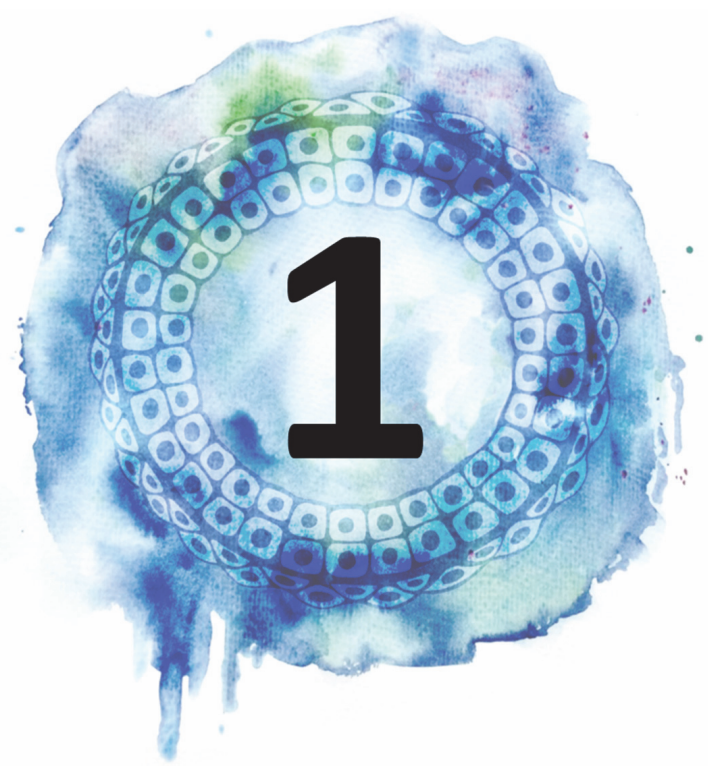

General Introduction 



\section{Background}

Modern sow lines have been genetically selected to increase litter size. In the Netherlands, in the period $2000-2017$, this genetic selection has successfully increased the number of piglets born alive from 11.2 to 14.8 and the number of piglets weaned from 10 to 12.9 (Agrovision B.V., Deventer, The Netherlands). Sows farrow around 2.4 litters per year, which results in an average production of 30 weaned piglets per sow per year. However, next to the successful increase in litter size, the number of stillborn piglets increased from 0.9 to 1.3 per litter, as did the percentage of liveborn piglets that died before weaning, which has increased from 10.7 to 12.9\% (Agrovision B.V., depicted in Fig. 1.1). This higher piglet mortality rate has negative consequences for pig welfare (Rutherford et al., 2013), which is of growing concern to our society (Grandin, 2014), but also strongly impacts pig production profitability. The latter is of high societal relevance as pork is the most widely consumed meat type worldwide ( $37 \%$ of the total amount of consumed meat according to the Food and Agriculture Organization of the United Nations), and meat consumption is rising due to increasing average individual incomes and the steadily growing world population (Godfray et al., 2018).

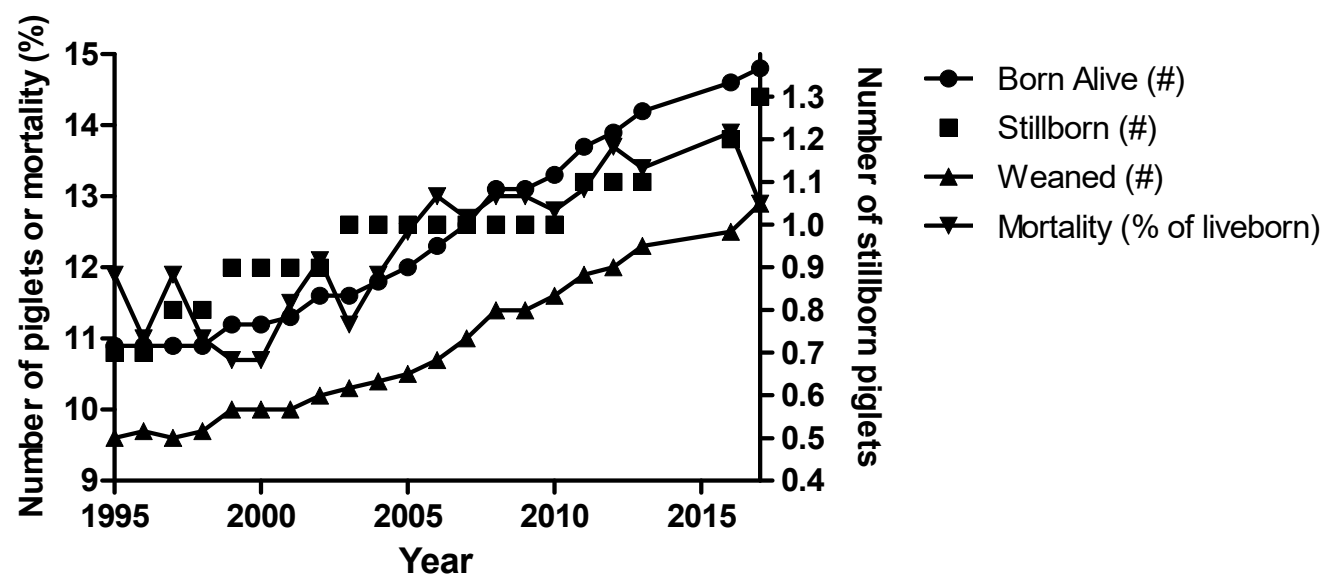

Figure 1.1 - Number of piglets born alive, stillborn, weaned and percentage mortality of liveborn piglets per litter in the Netherlands from 1995 to 2017 (Agrovision B.V.).

The increased pre-weaning piglet mortality can be partly explained by lower piglet birth weights and higher within-litter variation in birth weight (Milligan et al., 2002; Tuchscherer et al., 2000). In large litters, average piglet birth weight is decreased while within-litter variation in birth weight is increased (Quesnel et al., 2008; Quiniou et al., 2002; Wientjes et al., 2012; Wolf et al., 2008). Piglet birth weight has been shown to decrease with 35 grams for each additional piglet if litters increase in size from $\leq 11$ to $\geq 16$ piglets (Quiniou et al., 2002). A decrease with 40 grams for each additional piglet has been observed in litters from organic farms (Wientjes et al., 2012). Within-litter variation in birth weight (expressed as coefficient of variation) increased from 15 to $24 \%$ in litter sizes of $\leq 10$ vs. $\geq 15$, respectively (Quesnel et 
al., 2008) and increased with $0.75 \%$ for each additional piglet (Wientjes et al., 2012). Next to the effect of low piglet birth weights and high within-litter variation in birth weight on preweaning piglet mortality, low piglet birth weights can also have consequences for piglet development, such as lower weights at weaning, slower growth after weaning and reduced meat and carcass quality (e.g. Beaulieu et al., 2010; Gondret et al., 2005; Rehfeldt et al., 2008). This can further limit pig production profitability (Fix et al., 2010). It is up to now not clear what drives low piglet birth weights and higher variation in piglet birth weight.

Due to high metabolic demands for milk production, sows usually experience a negative energy balance (NEB) during lactation. With the increase in litter size over the last decades, metabolic demands of sows and thus the chance to enter a substantial NEB during lactation, has increased due to higher milk production. When lactational weight loss exceeds $10-12 \%$ of initial body weight at parturition, the weight loss of the sow is associated with lower subsequent reproductive performance in terms of farrowing rates, weaning-to-service intervals and litter size (Schenkel et al., 2010; Thaker and Bilkei, 2005). Moreover, more weight loss during lactation reduces embryo weight and survival during the next pregnancy (Hoving et al., 2012; Patterson et al., 2011; Vinsky et al., 2006), and increases within-litter variation in piglet birth weight (Wientjes et al., 2013). The negative effects of the lactational NEB on piglet birth weights may be explained by the influence of the pre-mating metabolic state, during and after lactation, on the development of follicles that will give rise to the next litter (reviewed by: Prunier and Quesnel, 2000). Feed restriction during lactation has been shown to result in smaller follicles at weaning and 48 hours later during the next follicular phase (Quesnel et al., 1998) and decreased in vitro oocyte maturation rates when isolated 38 hours before the anticipated onset of oestrus (Zak et al., 1997). We thus hypothesise that low piglet birth weights are (at least partly) explained by differences in follicular development. More evidence for this hypothesis comes from early studies by Pope et al. $(1988,1989)$ and Xie et al. (1990), who have established a positive relation between follicle diversity and embryonic diversity. In turn, embryonic size and diversity has been related to piglet birth weight and variation in piglet birth weight (Van der Lende et al., 1990). Early embryonic development is an important phase in post-fertilization development because it determines placenta size, size of the implantation site in the uterus and nutrient supply to the embryo (reviewed by Fowden et al., 2009). A more detailed analysis of follicular development during lactation and around weaning may offer additional information to identify to which extent and how differences in follicular development can contribute to lower piglet birth weights, higher within litter variation in piglet birth weight and piglet survival. 


\section{Follicular development}

Ovarian follicular recruitment can be subdivided in two phases that are differently controlled. Initial follicular development is initiated during foetal life, when oogonia start to multiply and subsequently enter the prophase of the first meiotic division and become surrounded by flat pre-granulosa cells. These so-called primordial follicles form the only available source of oocytes for the entire duration of reproductive life. During reproductive life, primordial follicles are continuously recruited to start development and oocytes become surrounded by multiple layers of granulosa cells and theca cells (reviewed by McGee and Hsueh, 2000). In this stage of follicular development, follicular growth and development is mainly regulated via locally produced growth factors in a time and species dependent manner (reviewed by Juengel and McNatty, 2005). Once the follicles have developed to the late pre-antral/early antral stage (depending on the species), the responsiveness of follicles to gonadotropic hormones increases. Follicles now become critically dependent on the gonadotropin follicle-stimulating hormone (FSH) for their growth, as is shown by an FSH-induced increase in the expression of the proliferation marker PCNA (Yu et al., 2005). In addition, the gonadotropins FSH and luteinizing hormone (LH) stimulate follicular steroidogenesis. According to the classical twocell two-gonadotropin concept, theca cells are mainly stimulated by $\mathrm{LH}$ to produce androgens, which can be transformed to oestrogens by the enzyme aromatase (CYP19A1) produced by granulosa cells after activation by FSH (reviewed by Palermo, 2007). The time span needed for development from primordial to antral follicle stages is estimated to be about 84 days in the sow (Morbeck et al., 1992). The continuous recruitment of primordial follicles results in a pool of late pre-antral/small antral follicles in the ovaries at any given stage of life from puberty onwards.

The start of the second phase of follicular recruitment, so-called cyclic recruitment from the antral follicle pool, is initiated by a shift in hypothalamic gonadotropin releasing hormone $(\mathrm{GnRH})$ and LH pulse frequency, from low frequency high amplitude pulses to high frequency low amplitude pulses. In cyclic pigs, this occurs after luteolysis and the concomitant reduction in progesterone levels while in productive sows, this occurs at weaning of the piglets by removal of the suckling inhibition on follicular development. During cyclic recruitment a group of the existing relatively small antral follicles, which in pigs are around 3-4 $\mathrm{mm}$ in size (reviewed by Lucy et al., 2001 and McGee and Hsueh, 2000), are stimulated to grow. At this stage, the oocyte has completed its growth and is now competent to complete meiosis (reviewed by Masui and Clarke, 1979). Granulosa cells acquire LH receptor (LHCGR) under the influence of FSH, which enables $\mathrm{LH}$ to induce differentiation and inhibit proliferation of granulosa cells (Robker and Richards, 1998). The timing of LHCGR expression in granulosa cells is species specific, as human granulosa cells already acquire LHCGR at the start of the follicular phase of the menstrual cycle (Yung et al., 2014), while in rats and mice, LHCGR is only expressed in the granulosa cells of pre-ovulatory follicles (Teerds and Dorrington, 1995). The exact onset of granulosa cell LHCGR expression during follicular development in pigs is not 
clear. The recruited antral follicles produce more and more oestrogen and inhibin, which both exert a negative feedback on FSH production. This results in suppression of growth and atresia of smaller follicles with low responsiveness to FSH and lower numbers of LH receptors (reviewed by Knox, 2005). At the same time, larger follicles with sufficient LH receptors are able to respond to the pulsatile LH release and continue to grow. Possibly also other mechanisms for selection of follicles that develop further play a role. For mono-ovulatory species such as bovine, selection is also dependent on e.g. differential regulation of blood vessel formation and insulin-like growth factor 1 (IGF1) signalling which can rescue dominant follicles from atresia (reviewed by Acosta, 2007 and Fortune et al., 2004). Selected follicle(s) will finally ovulate when granulosa cell oestrogen production reaches a certain threshold, inducing the LH surge and ovulation at approximately $30 \pm 3 \mathrm{~h}$ after the peak in LH levels in sows (Soede et al., 1994). The total number of ovulated follicles is dependent on the number of follicles that respond to the shift in LH pulse frequency at the onset of the follicular phase, in other words the number of recruited follicles which have sufficient $\mathrm{LH}$ receptors to be selected (reviewed by Guthrie, 2005 and Lucy et al., 2001). From literature it is known that the preovulatory follicle pool and its corresponding follicular fluid are very heterogeneous in size and steroid content, respectively (Foxcroft et al., 1987; Hunter and Wiesak, 1989). This follicular heterogeneity is also found in human pre-ovulatory follicles (Carpintero et al., 2015). To which extent this variety contributes to the developmental competence of the follicles is not clear. In sows, the entire process of cyclic follicular recruitment and selection until ovulation, takes approximately 5 days from weaning onwards. The high oestrogen levels do not only trigger the LH surge, but also initiate oestrus behaviour, during which sows can be inseminated and fertilization can take place. An overview of follicles in different developmental stages can be found in Fig. 1.2.
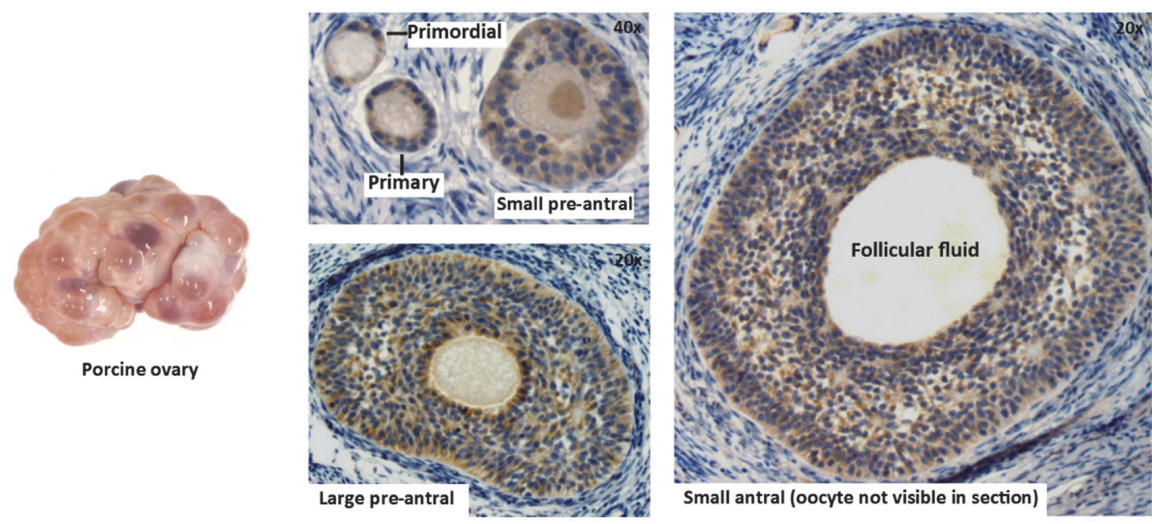

Figure 1.2 - Overview of follicular development in sows, showing a photograph of a porcine ovary (obtained $48 \mathrm{~h}$ after weaning from a primiparous sow) and histological images of follicles in different stages of follicular development; primordial, primary, small pre-antral, large pre-antral and small antral. 


\section{Granulosa cells}

Granulosa cells play an essential role in follicular development, as they express growth factor and gonadotropin receptors and produce growth factors and steroids needed for follicular growth and survival (reviewed by Matsuda et al., 2012). In addition, granulosa cells provide the oocyte with the necessary nutrients and metabolic intermediates allowing the oocytes to obtain their full developmental competence (Jiang et al., 2010; Sugiura et al., 2005). Granulosa cells consist of two subtypes with distinct functions; the mural granulosa cells which line the follicle wall and are mainly responsible for follicular steroidogenesis together with the theca cells, and the cumulus granulosa cells which surround the oocyte and provide the oocyte with the necessary growth factors and nutrients (Fig. 1.3). Reciprocally, the oocyte plays a crucial role in determining the fate of the follicular somatic cells via the secretion of oocyte secreted factors (OSFs) (Li et al., 2000). These OSFs include growth differentiation factors (GDFs) and bone morphogenetic proteins (BMPs), which mainly signal via growth factor receptors on granulosa cells, such as transforming growth factor beta-receptor 2 (TGFBR2) (Gilchrist et al., 2006). As a response to the OSFs, the granulosa cells produce their own paracrine factors, such as kit ligand, inhibins, activins, transforming growth factor alpha (TGFA) and antiMüllerian hormone (AMH) (reviewed by Oktem and Urman, 2010). The gradient of the OSFs determines the phenotypic fate of the granulosa cells, as the cumulus granulosa cells are exposed to higher levels of OSFs as compared to the mural granulosa cells, which are not in direct contact with the oocyte. OSFs are very potent mitogens that also affect mural granulosa cell function, facilitating follicular growth by inducing granulosa cell proliferation (Brankin et al., 2003). This mitogenic action of OSFs is mediated via the combined action of many other paracrine factors and gonadotropins in a species and timing-specific manner. One of the signalling components in this network is the epidermal growth factor (EGF) receptor. This receptor can be activated by EGF-like growth factors, such as TGFA, epiregulin and EGF itself (Harris et al., 2003), increasing granulosa cell receptivity for the mitogenic action of OSFs (Sasseville et al., 2010). In later stages of antral follicular development, granulosa cell proliferation decreases, while granulosa cells mature and differentiate, marked by an increase in follicular steroid production (reviewed by Hsueh et al., 1984). In vitro studies show that this granulosa cell maturation is stimulated by $\mathrm{FSH}, \mathrm{LH}$, and factors which increase FSH receptor and LHCGR expression (Grieshaber et al., 2000) while maturation may be inhibited by OSFs such as GDF-9 and BMP-15 via suppression of FSH receptor expression (Otsuka et al., 2001; Vitt et al., 2000). 


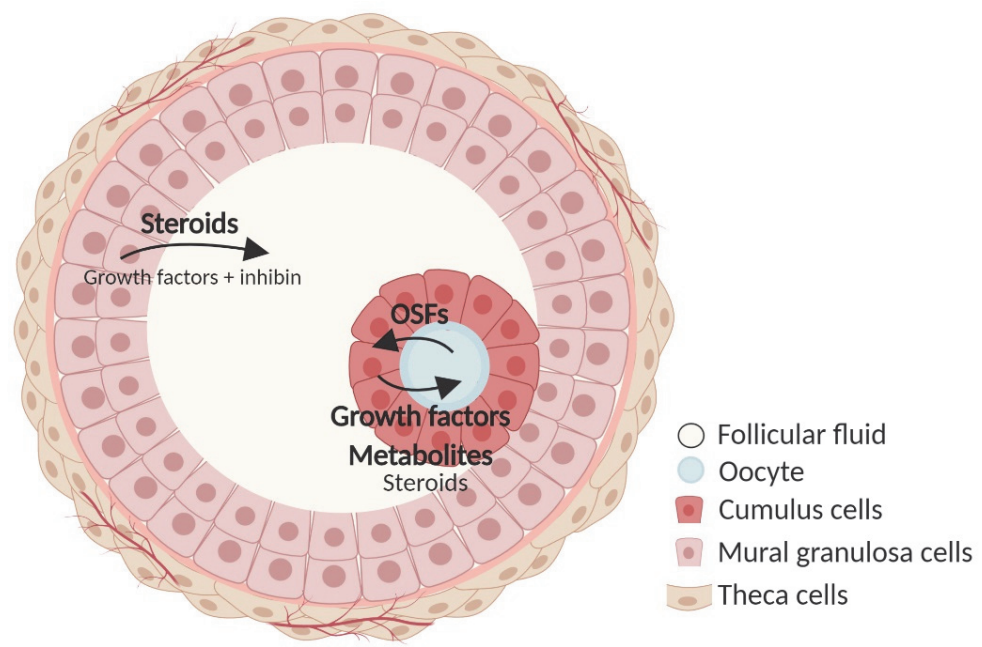

Figure 1.3 - Schematic overview of an antral follicle which consists of granulosa cells with distinct functions. The mural granulosa cells are, together with the theca cells, mainly responsible for steroidogenesis, while the cumulus cells are mainly responsible for providing the oocyte with the necessary growth factors and metabolites. Reciprocally, the oocytes produce oocyte secreted factors (OSFs) which stimulate follicular growth and survival and granulosa cell growth factor production. Together, the oocyte and cumulus cells form the cumulus-oocyte complex (COC). This illustration was made using BioRender.

\section{Mural granulosa cells}

Mural granulosa cells are, together with the theca interna cells, mainly responsible for follicular steroidogenesis. Steroidogenesis is initiated via the import of cholesterol into the mitochondria via steroidogenic acute regulatory protein (STAR). Inside the mitochondria, cholesterol is converted to pregnenolone via cytochrome P450 family 11 sub family A member 1 (CYP11A1) enzyme. The formed steroid pregnenolone moves from the mitochondria to the smooth endoplasmic reticulum in the cytoplasm where it can be converted into progesterone or dehydroepiandrosterone (DHEA) and androstenedione via 3-beta-hydroxysteroid dehydrogenase (HSD3B) and cytochrome P450 family 17 subfamily A member 1 (CYP17A1), respectively. DHEA and androstenedione can finally be converted into the sex steroids, testosterone and estradiol (Fig. 1.4).

Follicular steroid production is essential for oocyte developmental competence. As explained below in more detail, both estradiol and progesterone can increase oocyte developmental competence during in vitro maturation (IVM) in a temporal manner (e.g. Ballester et al., 2014; Yuan et al., 2016). Similarly, high progesterone and androstenedione levels stimulate oocyte developmental competence, as shown by increased in vitro cleavage and blastocyst formation rates after IVM with follicular fluid from adult sows vs. gilts, the former having higher progesterone and androstenedione levels (Grupen et al., 2003). In early antral follicles, mural granulosa cells are mainly responsible for $\mathrm{FSH}$-induced aromatase production, which converts androgens, produced by the neighbouring theca cells, into estradiol. 


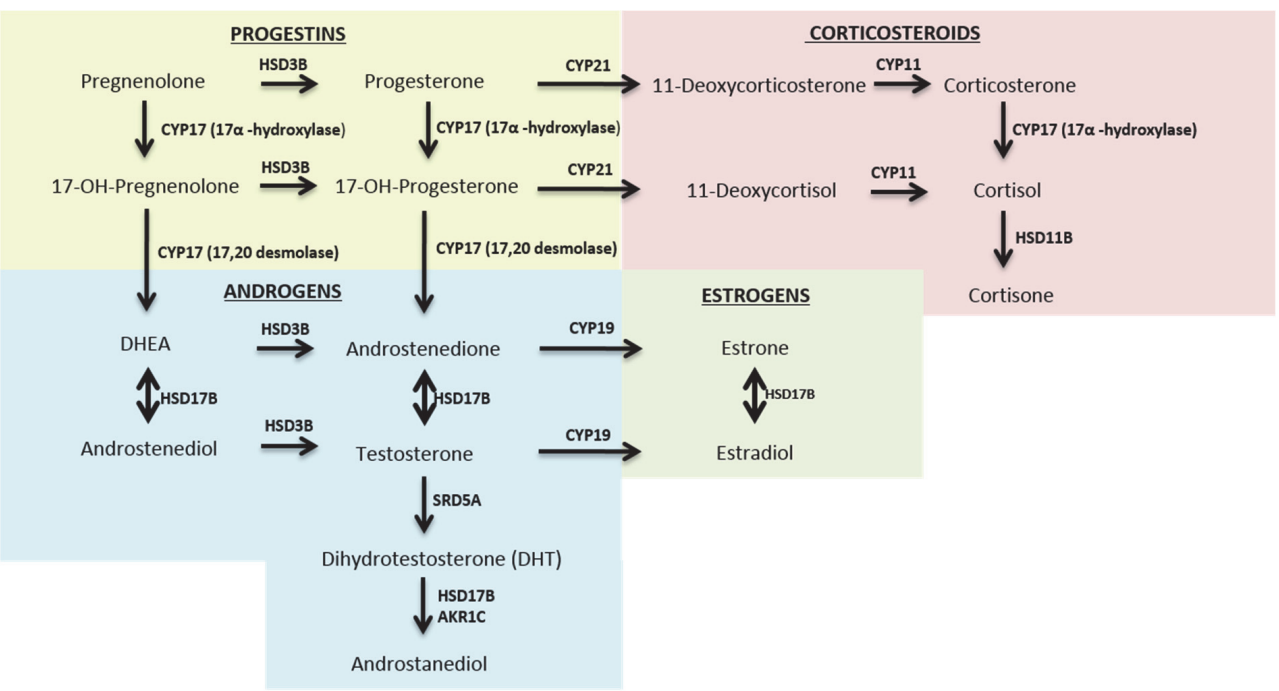

Figure 1.4 - Schematic overview of the steroidogenic pathway for the synthesis of progestins, androgens, estrogens and corticosteroids (adapted from Boron and Boulpaep, 2016). CYP17 =

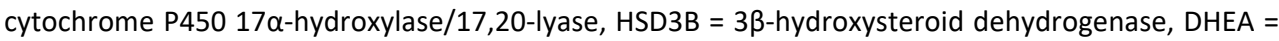
dehydroepiandrosterone, CYP21 = cytochrome P450 family 21, CYP11 = cytochrome P450 family 11 , HSD11B = 11 $\beta$-hydroxysteroid dehydrogenase, HSD17B = 17 $\beta$-hydroxysteroid dehydrogenase, CYP19 $=$ aromatase, SRD5A $=5 \alpha$-reductase, AKR1C $=$ aldo-keto reductase $1 \mathrm{C}$.

However, in later stages of follicular development, the granulosa cells also start to express STAR and CYP11A1, which enables the granulosa cells to convert cholesterol into progesterone. Timing of granulosa cell STAR and CYP11A1 expression is species-specific. In gilts, altrenogest, a progesterone agonist which supresses $\mathrm{GnRH}$ and gonadotropin release, is used to synchronize oestrus cycles. Gilts are in oestrus around 5 to 7 days after altrenogest withdrawal. In gilts, minimal amounts of CYP11A and STAR mRNA and protein is expressed from day 3 or 5, respectively, after altrenogest withdrawal onwards (Garmey et al., 2000). In mice and rats, granulosa cells only express STAR and CYP11A from the pre-ovulatory follicle stage, around the time of the LH surge onwards, so in later stages of follicular development (reviewed by Payne and Hales, 2004; Ronen-Fuhrmann et al., 1998). For sows, it is not clear to which extent granulosa cells contribute to steroidogenesis and how this in turn contributes to follicular developmental competence.

\section{Cumulus cells}

While the mural granulosa cells are mainly responsible for steroid production, the cumulus cells are of particular importance for providing the oocyte with necessary nutrients and metabolic intermediates, as they are in direct contact with the oocyte. The cumulus cells communicate to the oocyte and vice-versa via gap junctions, consisting of connexin hexamers allowing the transit of molecules less than 1 kDa (Kidder and Mhawi, 2002), e.g. ATP, sodium, chloride and calcium ions and importantly, the secondary messenger CAMP, which regulates 
oocyte maturation (Luciano et al., 2004; Webb et al., 2002). Cumulus cells play a pivotal role in regulating oocyte energy availability and homeostasis, especially in the latest stages of follicular development, around the energy demanding process of oocyte maturation and ovulation. Oocytes obtain their energy from different substrates, e.g. glucose (mainly through its derivatives lactate and pyruvate), amino acids and lipids (Nandi et al., 2008). Oocytes have a limited capacity to form pyruvate from glucose and are therefore highly dependent on the cumulus cells, which produce large amounts of pyruvate (reviewed by Gu et al., 2015). Oocytes do have lactate dehydrogenase activity (Cetica et al., 1999; Yuan et al., 2011), allowing the conversion of pyruvate into lactate, which can subsequently be used as an alternative energy source for oocyte maturation. Next to this, oocytes also highly rely on cumulus cell fatty acid oxidation, especially around the energy demanding process of ovulation (Dunning et al., 2014). Finally, cumulus cells regulate oocyte amino acid uptake (Colonna and Mangia et al., 1983; Pelland et al., 2009).

\section{Metabolic influences on follicular development}

From the previously described processes, it becomes apparent that the process of follicular development requires energy and nutrients to facilitate follicular growth and maturation of the oocyte. Therefore, it is not surprising that relations between the metabolic state of an individual during follicular development and its reproductive performance have been found (e.g. Hoving et al., 2010; Moslehi et al., 2017; Wathes, 2012). This relation is especially evident in highly productive animal species, such as cows and pigs which can experience a severe NEB during lactation. This is also the time that maturation of the next pool of follicles occurs which potentially gives rise to the next offspring. During such a NEB, energy in the form of lipids and protein is mobilized from adipose and muscle tissue, to sustain milk production (Bergsma et al., 2009). Mobilization of body tissues is communicated on a whole-body level via the secretion of several metabolic factors and hormones. These can influence follicular development directly at the ovarian level but also indirectly via influencing gonadotropin release. Peripheral IGF1 and leptin levels indirectly influence follicular development by binding to insulin and IGF1, and leptin receptors, respectively, in the hypothalamus and pituitary. Lower IGF1 and leptin levels in a catabolic state can diminish the release of the gonadotropins FSH and LH (Odle et al., 2018; reviewed by Wolfe et al., 2014). In addition, both peripheral and granulosa cell produced IGF1 is essential for follicular development, as IGF1 can activate IGF1 signalling in granulosa cells and synergizes with FSH to stimulate granulosa cell proliferation and steroidogenesis (Liu et al., 2000; Xia et al., 1994). Leptin probably also has direct effects on the ovarian level, as in vitro experiments have shown that leptin has a role in the regulation of follicular fluid estradiol, androstenedione, and progesterone levels (Agarwal et al., 1999; Ruiz-Cortés et al., 2003; Spicer and Francisco, 1997). Despite these in vitro data the exact in vivo role of leptin in follicular development largely remains to be elucidated. Other metabolic intermediates known to directly influence follicular development on the ovarian 
level are non-esterified fatty acids (NEFA). During a negative energy balance, NEFA levels are elevated as a result of increased lipid metabolism (Bjerre-Harpøth et al., 2012; Lafontan and Langin, 2009; Schams et al., 1994). In bovine, in vitro observations have shown that addition of high levels of NEFA to the medium can inhibit granulosa cell proliferation and induce apoptosis (VanHolder et al., 2005).

As follicular fluid is an exudate of the serum, the metabolic composition reflects the wholebody metabolic state (Valckx et al., 2012) as well as the metabolic activity of the follicle itself (Fortune et al., 2004; Gérard et al., 2015). Follicular fluid metabolomics has allowed identification of metabolites that may directly affect follicular development and follicular competence. A study involving human ovarian tissue showed that the follicular fluid of follicles of which the oocytes could be fertilized and the developing embryos were capable of implantation, contained less glucose, more lactate and branched chain amino acids, compared to follicles with less competent oocytes (Wallace et al., 2012). In line with these observations, follicular fluid of follicles of which the oocytes successfully developed into multi-cellular embryos had a lower saturated/unsaturated fatty acid ratio as compared to follicular fluid of less competent oocytes which failed to cleave following fertilization (O'Gorman et al., 2013). Despite these examples limited information is available on the composition of the follicular fluid in relation to follicle and oocyte quality. Such information would provide more insight in the processes that determine follicular competence and subsequent reproductive success.

\section{Research gaps}

To conclude, reproductive problems in sows, such as the previously described trend towards lower piglet birth weights and associated pre-weaning mortality in highly prolific sows, may originate (at least partly) from impairments in the antral follicle pool from which follicles are selected to become ovulatory follicles, potentially giving rise to the next litter. The underlying processes, which determine, and influence follicle developmental competence remain poorly understood. Although previous studies have attempted to identify physiological characteristics of follicular developmental competence, these studies had variable outcomes. The main physiological characteristics that were studied are antral follicle size, where larger antral follicles either might yield better quality oocytes with improved blastocyst formation and implantation rates (Lequarre et al., 2005; Seneda et al., 2001), as well as morphological assessment of cumulus-oocyte complexes (COC) (Nagano et al., 2006; Mikkelsen and Lindenberg, 2001) and follicular fluid steroid content (Grupen et al., 2003; Kawashima et al., 2008). However, while some of these follicular characteristics have been related to follicular developmental competence, other studies were not able to confirm these relations (reviewed by Balaban and Urman, 2006 and Wang and Sun, 2006). One of the factors which may explain these variable outcomes, is that most animal studies make use of ovaries from the abattoir, where follicles are usually pooled in different size classes and the phase of the oestrus cycle in which the ovaries were obtained is not well controlled. These types of studies may therefore 
be less suitable to provide insight in follicular characteristics that determine developmental competence. In addition, most studies investigate the follicle pool as a whole, without considering the health status of the individual follicles. Over $99 \%$ of the follicles will become atretic and degenerate at some point during follicular development, and follicles may still become atretic in the latest stages of development when cyclic recruitment is already initiated (reviewed by Manabe et al., 2004). Results of studies which do not consider follicular health status may have less predictive value for oocyte developmental competence and consequently, for reproductive outcome. This stresses the necessity to study follicular characteristics which determine follicular developmental competence, while taking into consideration the developmental stage of the follicle pool and health status of the individual follicles studied.

Another important aspect is the metabolic state of the sows during lactation as it highly influences follicular development around weaning (Quesnel et al., 1998; Zak et al. 1997). However, most studies which have investigated this in sows have been performed over 20 years ago. Since that time, metabolic demands of sows during lactation have considerably increased. This is due to increased milk production to feed the higher number of piglets, and may be further due to lower feed intakes, as selection for feed efficiency has been associated with lower feed intakes (Bergsma et al., 2009; Kanis, 1990). Together, this may result in increased body reserve mobilization during lactation. In addition, modern sows of high genetic merit have a higher capacity for gain of lean mass and a lower gain of adipose tissue as compared to older lines and breeds. This may have consequences for metabolism of modern sows during lactation. It is therefore important to study effects of the metabolic state during lactation on follicular development and competence, in modern highly prolific sows. This may result in new management strategies to improve reproductive outcome in modern sows. In addition, these studies will lead to more insight in important follicular characteristics and processes that determine follicular competence and how these are affected by environmental influences.

\section{Aims and outline of the thesis}

Elucidating physiological characteristics which determine follicular developmental competence and understanding how follicular development is influenced by the metabolic state of the sow, could increase our understanding of the origin of reproductive problems in pigs and other mammals. Therefore, the aims of this thesis were:

1) To establish physiological and molecular characteristics of follicular developmental competence in sows by unravelling physiological processes that correlate with antral follicle size and oocyte health.

2) To better understand physiological relations between the metabolic state of sows and follicular developmental competence. 
In this thesis, follicular developmental competence is defined as the competence of a follicle, to yield a mature oocyte which can successfully develop into healthy offspring after fertilization.

In Chapter 2 of this thesis, I compared follicular development of sows with a high vs. low estimated breeding value (EBV) for within-litter variation in birth weight, to investigate which follicular characteristics differ in sows with low or high within-litter variation in birth weights. In addition, I assessed relations between follicular development, the metabolic state of sows during lactation and metabolic parameters at weaning. In Chapter 3, I investigated granulosa cell processes that are related to follicle size in sows with low vs. high variation in follicle size within the follicle pool, as follicle size may be a potential marker for follicular developmental competence. In Chapter 4, I have studied follicular fluid steroids characteristic for follicular developmental competence, by studying relations between follicular fluid steroid profiles and oocyte health. In Chapter 5, I identified follicular fluid steroids that reflect follicular development in the early stages of the follicular phase and established whether follicular fluid steroid levels correspond to that in serum. If these relations are established, serum steroid profiles may be used to monitor follicular development. In Chapter 6, I have studied effects of feed restriction during lactation on follicular developmental competence. This may elucidate potential follicular characteristics that influence reproductive outcome, as feed restriction and more weight loss during lactation are known to result in reduced reproductive performance and litter characteristics in the next litter. Next to sow performance, also piglet performance may be affected by feed restriction and mobilization of body tissues of modern sows during lactation. This will be investigated in Chapter 7, where I will elucidate relations between the metabolic state of modern hybrid sows during lactation and milk production parameters. In Chapter $\mathbf{8}$ (the general discussion of this PhD thesis), findings from Chapter 2-7 are combined and further discussed to unravel physiological and molecular aspects of follicular developmental competence and to understand their potential consequences for reproductive outcome, not only for sows but also for other animal species. In addition, I will discuss relations between the metabolic state of sows and follicular developmental competence and its implications for new (nutritional) strategies to improve reproductive functioning in sows. This will be translated into general conclusions and recommendations. See Fig. 1.5 for a schematic overview of this PhD thesis. 


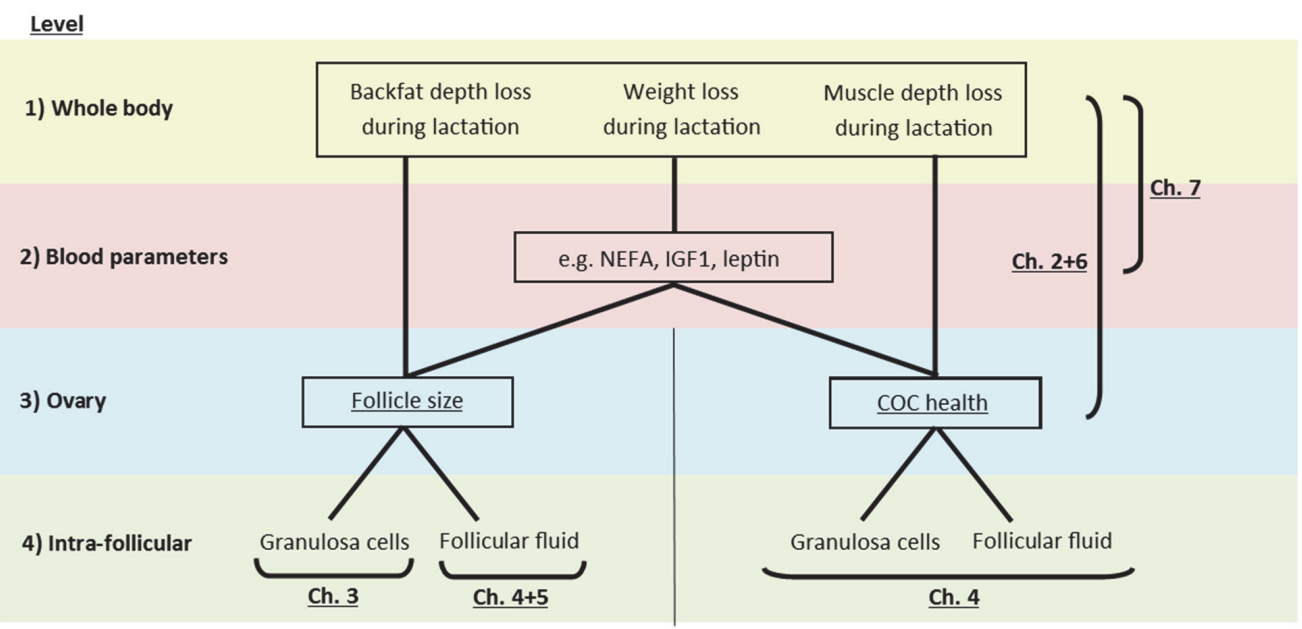

Figure 1.5 - Outline of this PhD thesis, in which I investigated: 1) molecular and physiological characteristics of ovarian follicular developmental competence in sows and 2) relations between the sows' metabolic state and ovarian follicular developmental competence.

\section{References}

Acosta, T. J. (2007). Studies of follicular vascularity associated with follicle selection and ovulation in cattle. Journal of reproduction and development, 53(1), 39-44.

Agarwal, S. K., Vogel, K., Weitsman, S. R., \& Magoffin, D. A. (1999). Leptin antagonizes the insulin-like growth factor-I augmentation of steroidogenesis in granulosa and theca cells of the human ovary. The journal of clinical endocrinology \& metabolism, 84(3), 1072-1076.

Ainsworth, L., Tsang, B., Downey, B., Marcus, G., \& Armstrong, D. (1980). Interrelationships between follicular fluid steroid levels, gonadotropic stimuli, and oocyte maturation during preovulatory development of porcine follicles. Biology of reproduction, 23(3), 621-627.

Balaban, B., \& Urman, B. (2006). Effect of oocyte morphology on embryo development and implantation. Reproductive biomedicine online, 12(5), 608-615.

Ballester, L., Romero-Aguirregomezcorta, J., Soriano-Úbeda, C., Matás, C., Romar, R., \& Coy, P. (2014). Timing of oviductal fluid collection, steroid concentrations, and sperm preservation method affect porcine in vitro fertilization efficiency. Fertility and sterility, 102(6), 1762-1768.

Beaulieu, A., Aalhus, J., Williams, N., \& Patience, J. (2010). Impact of piglet birth weight, birth order, and litter size on subsequent growth performance, carcass quality, muscle composition, and eating quality of pork. Journal of animal science, 88(8), 2767-2778.

Bergsma, R., Kanis, E., Verstegen, M., van der Peet-Schwering, C., \& Knol, E. (2009). Lactation efficiency as a result of body composition dynamics and feed intake in sows. Livestock science, 125(2), 208-222.

Bjerre-Harpøth, V., Friggens, N. C., Thorup, V. M., Larsen, T., Damgaard, B. M., Ingvartsen, K. L., \& Moyes, K. (2012). Metabolic and production profiles of dairy cows in response to decreased nutrient density to increase physiological imbalance at different stages of lactation. Journal of dairy science, 95(5), 2362-2380.

Boron, W. F., \& Boulpaep, E. L. (2016). In: Medical Physiology third edition (pp. 1116 - 1120). Elsevier: Philadephia, PA, USA.

Brankin, V., Mitchell, M. R., Webb, B., \& Hunter, M. G. (2003). Paracrine effects of oocyte secreted factors and stem cell factor on porcine granulosa and theca cells in vitro. Reproductive biology and endocrinology, 1(55). 
Carpintero, N. L., Suárez, O. A., Varea, C. G., Rioja, R. G., and Mangas, C. C. (2015). Variability between the follicular steroid hormone levels in different follicles of the same patient and between patients. Journal of human reproductive sciences, 8(1), 37-42.

Cetica, P., Pintos, L., Dalvit, G., \& Beconi, M. (1999). Effect of lactate dehydrogenase activity and isoenzyme localization in bovine oocytes and utilization of oxidative substrates on in vitro maturation. Theriogenology, 51(3), 541-550.

Colonna, R., and Mangia, F. (1983). Mechanisms of amino acid uptake in cumulus-enclosed mouse oocytes. Biology of reproduction, 28(4), 797-803.

Dunning, K. R., Russell, D. L., \& Robker, R. L. (2014). Lipids and oocyte developmental competence: the role of fatty acids and $\beta$-oxidation. Reproduction, 148(1), 15-27.

Fix, J., Cassady, J., Holl, J., Herring, W., Culbertson, M., \& See, M. (2010). Effect of piglet birth weight on survival and quality of commercial market swine. Livestock science, 132(1), 98-106.

Fortune, J., Rivera, G., \& Yang, M. (2004). Follicular development: the role of the follicular microenvironment in selection of the dominant follicle. Animal reproduction science, 82, 109126.

Fowden, A., Sferruzzi-Perri, A., Coan, P., Constancia, M., \& Burton, G. (2009). Placental efficiency and adaptation: endocrine regulation. The Journal of physiology, 587(14), 3459-3472.

Foxcroft, G.R., Shaw, H.J., Hunter, M.G., Booth, P.J., and Lancaster, R.T. (1987). Relationships between luteinizing hormone, follicle-stimulating hormone and prolactin secretion and ovarian follicular development in the weaned sow. Biology of reproduction, 36(1), 175-191.

Garmey, J. C., Guthrie, H., Garrett, W. M., Stoler, M. H., \& Veldhuis, J. D. (2000). Localization and expression of low-density lipoprotein receptor, steroidogenic acute regulatory protein, cytochrome P450 side-chain cleavage and P450 17- $\alpha$-hydroxylase/C17-20 lyase in developing swine follicles: in situ molecular hybridization and immunocytochemical studies. Molecular and cellular endocrinology, 170(1-2), 57-65.

Gérard, N., Fahiminiya, S., Grupen, C. G., \& Nadal-Desbarats, L. (2015). Reproductive physiology and ovarian folliculogenesis examined via $1 \mathrm{H}-\mathrm{NMR}$ metabolomics signatures: a comparative study of large and small follicles in three mammalian species (Bos taurus, Sus scrofa domesticus and Equus ferus caballus). Omics: a journal of integrative biology, 19(1), 31-40.

Gilchrist, R. B., Ritter, L. J., Myllymaa, S., Kaivo-Oja, N., Dragovic, R. A., Hickey, T. E., Ritvos, O., \& Mottershead, D. G. (2006). Molecular basis of oocyte-paracrine signalling that promotes granulosa cell proliferation. Journal of cell science, 119(18), 3811-3821.

Godfray, H. C. J., Aveyard, P., Garnett, T., Hall, J. W., Key, T. J., Lorimer, J., Pierrehumbert, R. T., Scarborough, P., Springmann, M., \& Jebb, S. A. (2018). Meat consumption, health, and the environment. Science, 361(6399).

Gondret, F., Lefaucheur, L., Louveau, I., Lebret, B., Pichodo, X., \& Le Cozler, Y. (2005). Influence of piglet birth weight on postnatal growth performance, tissue lipogenic capacity and muscle histological traits at market weight. Livestock production science, 93(2), 137-146.

Grandin, T. (2014). Animal welfare and society concerns finding the missing link. Meat science, 98(3), 461-469.

Grieshaber, N. A., Boitano, S., Ji, I., Mather, J. P., \& Ji, T. H. (2000). Differentiation of granulosa cell line: follicle-stimulating hormone induces formation of lamellipodia and filopodia via the adenylyl cyclase/cyclic adenosine monophosphate signal. Endocrinology, 141(9), 3461-3470.

Grupen, C. G., Mcllfatrick, S. M., Ashman, R. J., Boquest, A. C., Armstrong, D. T., \& Nottle, M. B. (2003). Relationship between donor animal age, follicular fluid steroid content and oocyte developmental competence in the pig. Reproduction, fertility and development, 15(2), 81-87.

Gu, L., Liu, H., Gu, X., Boots, C., Moley, K. H., \& Wang, Q. (2015). Metabolic control of oocyte development: linking maternal nutrition and reproductive outcomes. Cellular and molecular life sciences, 72(2), 251-271.

Guthrie, H. (2005). The follicular phase in pigs: Follicle populations, circulating hormones, follicle factors and oocytes. Journal of animal science 83, E79-E89. 
Harris, R. C., Chung, E., \& Coffey, R. J. (2003). "EGF receptor ligands." In The EGF Receptor Family (pp. 3-14). Academic Press, Burlington, UK.

Hoving, L. L., Soede, N. M., Graat, E. A., Feitsma, H., \& Kemp, B. (2010). Effect of live weight development and reproduction in first parity on reproductive performance of second parity sows. Animal reproduction science, 122(1-2), 82-89.

Hoving, L. L., Soede, N. M., Feitsma, H., \& Kemp, B. (2012). Lactation weight loss in primiparous sows: consequences for embryo survival and progesterone and relations with metabolic profiles. Reproduction in domestic animals, 47(6), 1009-1016.

Hsueh, A. J., Adashi, E., Jones, P. B., \& Welsh Jr, T. H. (1984). Hormonal regulation of the differentiation of cultured ovarian granulosa cells. Endocrine reviews, 5(1), 76-127.

Hunter, M., \& Wiesak, T. (1989). Evidence for and implications of follicular heterogeneity in pigs. Journal of reproduction and fertility. Supplement 40, 163-177.

Jiang, J.-Y., Xiong, H., Cao, M., Xia, X., Sirard, M.-A., \& Tsang, B. K. (2010). Mural granulosa cell gene expression associated with oocyte developmental competence. Journal of ovarian research, $3(6)$.

Juengel, J. L., \& McNatty, K. P. (2005). The role of proteins of the transforming growth factor- $\beta$ superfamily in the intraovarian regulation of follicular development. Human reproduction update, 11(2), 144-161.

Kanis, E. (1990). Effect of food intake capacity on production traits in growing pigs with restricted feeding. Animal science, 50(2), 333-341.

Kawashima, I., Okazaki, T., Noma, N., Nishibori, M., Yamashita, Y., \& Shimada, M. (2008). Sequential exposure of porcine cumulus cells to FSH and/or LH is critical for appropriate expression of steroidogenic and ovulation-related genes that impact oocyte maturation in vivo and in vitro. Reproduction, 136(1), 9-21.

Kidder, G. M., \& Mhawi, A. A. (2002). Gap junctions and ovarian folliculogenesis. Reproduction, 123(5), 613-620.

Knox, R. (2005). Recruitment and selection of ovarian follicles for determination of ovulation rate in the pig. Domestic animal endocrinology, 29(2), 385-397.

Lafontan, M., \& Langin, D. (2009). Lipolysis and lipid mobilization in human adipose tissue. Progress in lipid research, 48(5), 275-297.

Lequarre, A.-S., Vigneron, C., Ribaucour, F., Holm, P., Donnay, I., Dalbies-Tran, R., Callesen, H., \& Mermillod, P. (2005). Influence of antral follicle size on oocyte characteristics and embryo development in the bovine. Theriogenology, 63(3), 841-859.

Li, R., Norman, R. J., Armstrong, D. T., \& Gilchrist, R. B. (2000). Oocyte-secreted factor (s) determine functional differences between bovine mural granulosa cells and cumulus cells. Biology of reproduction, 63(3), 839-845.

Liu, J., Koenigsfeld, A. T., Cantley, T. C., Boyd, C. K., Kobayashi, Y., \& Lucy, M. C. (2000). Growth and the initiation of steroidogenesis in porcine follicles are associated with unique patterns of gene expression for individual componentsof the ovarian insulin-like growth factor system. Biology of reproduction, 63(3), 942-952.

Luciano, A. M., Modina, S., Vassena, R., Milanesi, E., Lauria, A., \& Gandolfi, F. (2004). Role of intracellular cyclic adenosine 3', 5'-monophosphate concentration and oocyte-cumulus cells communications on the acquisition of the developmental competence during in vitro maturation of bovine oocyte. Biology of reproduction, 70(2), 465-472.

Lucy, M., Liu, J., Boyd, C., \& Bracken, C. (2001). Ovarian follicular growth in sows. Reproduction. Supplement, 58, 31-45.

Manabe, N., Goto, Y., Matsuda-Minehata, F., Inoue, N., Maeda, A., Sakamaki, K., \& Miyano, T. (2004). Regulation mechanism of selective atresia in porcine follicles: regulation of granulosa cell apoptosis during atresia. Journal of reproduction and development, 50(5), 493-514.

Masui, Y., \& Clarke, H. J. (1979). "Oocyte maturation." In: International review of cytology (pp.185282). Academic Press, Burlington, UK. 
Matsuda, F., Inoue, N., Manabe, N., \& Ohkura, S. (2012). Follicular growth and atresia in mammalian ovaries: regulation by survival and death of granulosa cells. Journal of reproduction and development, 58(1), 44-50.

McGee, E. A., \& Hsueh, A. J. (2000). Initial and cyclic recruitment of ovarian follicles. Endocrine reviews, 21(2), 200-214.

Mikkelsen, A. L., \& Lindenberg, S. (2001). Morphology of in-vitro matured oocytes: impact on fertility potential and embryo quality. Human reproduction, 16(8), 1714-1718.

Morbeck, D. E., Esbenshade, K. L., Flowers, W. L., \& Britt, J. H. (1992). Kinetics of follicle growth in the prepubertal gilt. Biology of reproduction, 47(3), 485-491.

Moslehi, N., Mirmiran, P., Tehrani, F. R., \& Azizi, F. (2017). Current evidence on associations of nutritional factors with ovarian reserve and timing of menopause: a systematic review. Advances in nutrition, 8(4), 597-612.

Nagano, M., Katagiri, S., \& Takahashi, Y. (2006). Relationship between bovine oocyte morphology and in vitro developmental potential. Zygote, 14(1), 53-61.

Nandi, S., Kumar, V. G., Manjunatha, B., Ramesh, H., \& Gupta, P. (2008). Follicular fluid concentrations of glucose, lactate and pyruvate in buffalo and sheep, and their effects on cultured oocytes, granulosa and cumulus cells. Theriogenology, 69(2), 186-196.

Odle, A. K., Akhter, N., Syed, M. M., Allensworth-James, M. L., Beneš, H., Melgar Castillo, A. I., MacNicol, A. M., \& Childs, G. V. (2018). Leptin regulation of Gonadotrope Gonadotropin-releasing Hormone receptors as a Metabolic Checkpoint and Gateway to reproductive Competence. Frontiers in endocrinology, 8, 367.

O'Gorman, A., Wallace, M., Cottell, E., Gibney, M. J., McAuliffe, F. M., Wingfield, M., \& Brennan, L. (2013). Metabolic profiling of human follicular fluid identifies potential biomarkers of oocyte developmental competence. Reproduction, 146(4), 389-395.

Oktem, O., \& Urman, B. (2010). Understanding follicle growth in vivo. Human reproduction, 25(12), 2944-2954.

Otsuka, F., Yamamoto, S., Erickson, G. F., and Shimasaki, S. (2001). Bone morphogenetic protein-15 inhibits follicle-stimulating hormone (FSH) action by suppressing FSH receptor expression. Journal of biological chemistry 276, 11387-11392.

Palermo, R. (2007). Differential actions of FSH and LH during folliculogenesis. Reproductive biomedicine online, 15(3), 326-337.

Patterson, J., Smit, M., Novak, S., Wellen, A., \& Foxcroft, G. (2011). Restricted feed intake in lactating primiparous sows. I. Effects on sow metabolic state and subsequent reproductive performance. Reproduction, fertility and development, 23(7), 889-898.

Payne, A. H., \& Hales, D. B. (2004). Overview of steroidogenic enzymes in the pathway from cholesterol to active steroid hormones. Endocrine reviews, 25(6), 947-970.

Pelland, A. M., Corbett, H. E., and Baltz, J. M. (2009). Amino acid transport mechanisms in mouse oocytes during growth and meiotic maturation. Biology of reproduction 81, 1041-1054.

Pope, W., Wilde, M., \& Xie, S. (1988). Effect of electrocautery of nonovulated day 1 follicles on subsequent morphological variation among day 11 porcine embryos. Biology of reproduction, 39(4), 882-887.

Pope, W., Xie, S., Broermann, D., \& Nephew, K. (1989). Causes and consequences of early embryonic diversity in pigs. Journal of reproduction and fertility. Supplement, 40, 251-260.

Prunier, A., \& Quesnel, H. (2000). Influence of the nutritional status on ovarian development in female pigs. Animal reproduction science, 2(60-61), 185-197.

Quesnel, H., Pasquier, A., Mounier, A., \& Prunier, A. (1998). Influence of feed restriction during lactation on gonadotropic hormones and ovarian development in primiparous sows. Journal of animal science, 76(3), 856.

Quesnel, H., Brossard, L., Valancogne, A., \& Quiniou, N. (2008). Influence of some sow characteristics on within-litter variation of piglet birth weight. Animal, 2(12), 1842-1849.

Quiniou, N., Dagorn, J., \& Gaudré, D. (2002). Variation of piglets' birth weight and consequences on subsequent performance. Livestock production science, 78(1), 63-70. 
Rehfeldt, C., Tuchscherer, A., Hartung, M., \& Kuhn, G. (2008). A second look at the influence of birth weight on carcass and meat quality in pigs. Meat Science, 78(3), 170-175.

Robker, R. L., \& Richards, J. S. (1998). Hormone-induced proliferation and differentiation of granulosa cells: a coordinated balance of the cell cycle regulators cyclin D2 and p27Kip1. Molecular endocrinology, 12(7), 924-940.

Ronen-Fuhrmann, T., Timberg, R., King, S. R., Hales, K. H., Hales, D. B., Stocco, D. M., \& Orly, J. (1998). Spatio-temporal expression patterns of steroidogenic acute regulatory protein (STAR) during follicular development in the rat ovary. Endocrinology, 139(1), 303-315.

Ruiz-Cortés, Z. T., Martel-Kennes, Y., Gévry, N. Y., Downey, B. R., Palin, M.-F., \& Murphy, B. D. (2003). Biphasic effects of leptin in porcine granulosa cells. Biology of reproduction, 68(3), 789-796.

Rutherford, K., Baxter, E., D'eath, R., Turner, S., Arnott, G., Roehe, R., Ask, B., Sandøe, P., Moustsen, V., and Thorup, F. (2013). The welfare implications of large litter size in the domestic pig I: biological factors. Animal welfare 22(2), 199-218.

Sasseville, M., Ritter, L. J., Nguyen, T. M., Liu, F., Mottershead, D. G., Russell, D. L., \& Gilchrist, R. B. (2010). Growth differentiation factor 9 signaling requires ERK1/2 activity in mouse granulosa and cumulus cells. Journal of cell science, 123(18), 3166-3176.

Schams, D., Kraetzl, W.-D., Brem, G., \& Graf, F. (1994). Secretory pattern of metabolic hormones in the lactating sow. Experimental and clinical endocrinology \& diabetes, 102(06), 439-447.

Schenkel, A. C., Bernardi, M. L., Bortolozzo, F. P., \& Wentz, I. (2010). Body reserve mobilization during lactation in first parity sows and its effect on second litter size. Livestock science, 132(1-3), 165-172.

Seneda, M. M., Esper, C. R., Garcia, J. M., de Oliveira, J. A., \& Vantini, R. (2001). Relationship between follicle size and ultrasound-guided transvaginal oocyte recovery. Animal reproduction science, 67(1-2), 37-43.

Soede, N., Helmond, F., \& Kemp, B. (1994). Periovulatory profiles of oestradiol, LH and progesterone in relation to oestrus and embryo mortality in multiparous sows using transrectal ultrasonography to detect ovulation. Journal of reproduction and fertility, 101(3), 633-641.

Spicer, L. J., \& Francisco, C. C. (1997). The adipose obese gene product, leptin: evidence of a direct inhibitory role in ovarian function. Endocrinology, 138(8), 3374-3379.

Sugiura, K., Pendola, F. L., \& Eppig, J. J. (2005). Oocyte control of metabolic cooperativity between oocytes and companion granulosa cells: energy metabolism. Developmental biology, 279(1), 20-30.

Teerds, K. J., \& Dorrington, J. H. (1995). Immunolocalization of transforming growth factor a and luteinizing hormone receptor in healthy and atretic follicles of the adult rat ovary. Biology of reproduction, 52(3), 500-508.

Thaker, M. Y. C., \& Bilkei, G. (2005). Lactation weight loss influences subsequent reproductive performance of sows. Animal reproduction science, 88(3-4), 309-318.

Tuchscherer, M., Puppe, B., Tuchscherer, A., \& Tiemann, U. (2000). Early identification of neonates at risk: traits of newborn piglets with respect to survival. Theriogenology, 54(3), 371-388.

Valckx, S., De Pauw, I., De Neubourg, D., Inion, I., Berth, M., Fransen, E., Bols, P. E., \& Leroy, J. (2012). BMI-related metabolic composition of the follicular fluid of women undergoing assisted reproductive treatment and the consequences for oocyte and embryo quality. Human reproduction, 27(12), 3531-3539.

Van der Lende, T., Hazeleger, W., \& De Jager, D. (1990). Weight distribution within litters at the early foetal stage and at birth in relation to embryonic mortality in the pig. Livestock production science, 26(1), 53-65.

Vanholder, T., Leroy, J., Van Soom, A., Opsomer, G., Maes, D., Coryn, M., \& de Kruif, A. (2005). Effect of non-esterified fatty acids on bovine granulosa cell steroidogenesis and proliferation in vitro. Animal reproduction science, 87(1-2), 33-44.

Vinsky, M., Novak, S., Dixon, W., Dyck, M., \& Foxcroft, G. (2006). Nutritional restriction in lactating primiparous sows selectively affects female embryo survival and overall litter development. Reproduction, fertility and development, 18(3), 347-355. 
Vitt, U., Hayashi, M., Klein, C., \& Hsueh, A. (2000). Growth differentiation factor-9 stimulates proliferation but suppresses the follicle-stimulating hormone-induced differentiation of cultured granulosa cells from small antral and preovulatory rat follicles. Biology of reproduction, 62(2), 370-377.

Wallace, M., Cottell, E., Gibney, M. J., McAuliffe, F. M., Wingfield, M., \& Brennan, L. (2012). An investigation into the relationship between the metabolic profile of follicular fluid, oocyte developmental potential, and implantation outcome. Fertility and sterility, 97(5), 1078-1084.

Wang, Q., \& Sun, Q.-Y. (2006). Evaluation of oocyte quality: morphological, cellular and molecular predictors. Reproduction, fertility and development, 19(1), 1-12.

Wathes, D. (2012). Mechanisms linking metabolic status and disease with reproductive outcome in the dairy cow. Reproduction in domestic animals, 47, 304-312.

Webb, R., Bains, H., Cruttwell, C., \& Carroll, J. (2002). Gap-junctional communication in mouse cumulus-oocyte complexes: implications for the mechanism of meiotic maturation. Reproduction, 123(1), 41-52.

Wientjes, J. G. M., Soede, N. M, Van der Peet-Schwering, C. M. C., Van Den Brand, H., \& Kemp, B. (2012). Piglet uniformity and mortality in large organic litters: Effects of parity and pre-mating diet composition. Livestock science, 144(3), 218-229.

Wientjes, J. G. M., Soede, N. M., Knol, E. F., Van den Brand, H., \& Kemp, B. (2013). Piglet birth weight and litter uniformity: Effects of weaning-to-pregnancy interval and body condition changes in sows of different parities and crossbred lines. Journal of animal science, 91(5), 2099-2107.

Wolf, J., Žáková, E., \& Groeneveld, E. (2008). Within-litter variation of birth weight in hyperprolific Czech Large White sows and its relation to litter size traits, stillborn piglets and losses until weaning. Livestock science, 115(2-3), 195-205.

Wolfe, A., Divall, S., \& Wu, S. (2014). The regulation of reproductive neuroendocrine function by insulin and insulin-like growth factor-1 (IGF-1). Frontiers in neuroendocrinology, 35(4), 558-572.

Xia, P., Tekpetey, F. R., \& Armstrong, D. T. (1994). Effect of IGF-I on pig oocyte maturation, fertilization, and early embryonic development in vitro, and on granulosa and cumulus cell biosynthetic activity. Molecular reproduction and development, 38(4), 373-379.

Xie, S., Broermann, D., Nephew, K., Bishop, M., \& Pope, W. (1990). Relationship between oocyte maturation and fertilization on zygotic diversity in swine. Journal of animal science, 68(7), 2027-2033.

Yu, F.-Q., Han, C.-S., Yang, W., Jin, X., Hu, Z.-Y., \& Liu, Y.-X. (2005). Role of ERK1/2 in FSH induced PCNA expression and steroidogenesis in granulosa cells. Frontiers in bioscience, 10, 896-904.

Yuan, B., Liang, S., Jin, Y.-X., Kwon, J.-W., Zhang, J.-B., \& Kim, N.-H. (2016). Progesterone influences cytoplasmic maturation in porcine oocytes developing in vitro. PeerJ, 4, e2454.

Yuan, Y., Ida, J. M., Paczkowski, M., \& Krisher, R. L. (2011). Identification of developmental competence-related genes in mature porcine oocytes. Molecular reproduction and development, 78(8), 565-575.

Yung, Y., Aviel-Ronen, S., Maman, E., Rubinstein, N., Avivi, C., Orvieto, R., \& Hourvitz, A. (2014). Localization of luteinizing hormone receptor protein in the human ovary. Molecular human reproduction, 20(9), 844-849.

Zak, L., Xu, X., Hardin, R., \& Foxcroft, G. (1997). Impact of different patterns of feed intake during lactation in the primiparous sow on follicular development and oocyte maturation. Journal of reproduction and fertility, 110(1), 99-106. 



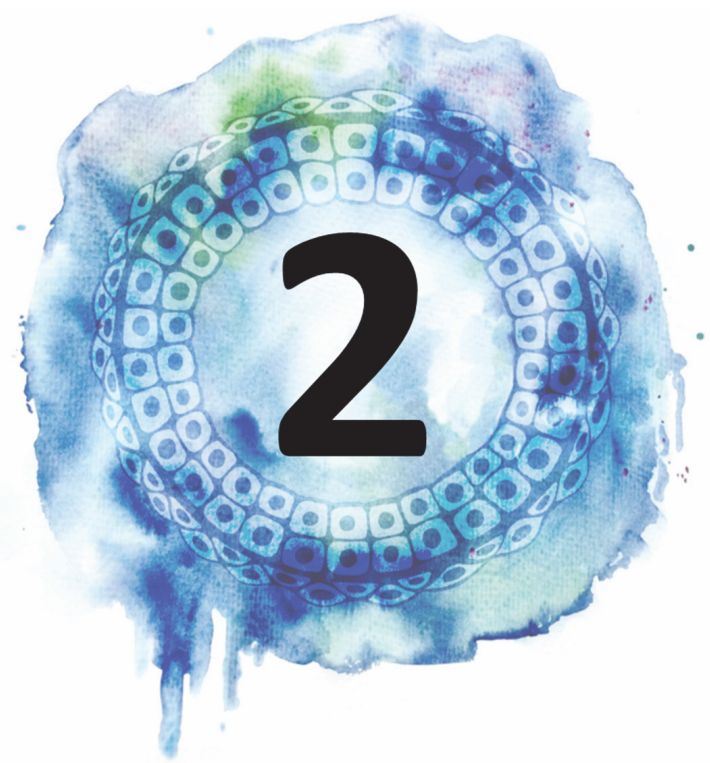

\title{
Follicular development of sows at weaning in relation to estimated breeding value for within-litter variation in piglet birth weight
}

\author{
Natasja G. J. Costermans ${ }^{1,2}$, Katja J. Teerds ${ }^{1}$, Jaap Keijer ${ }^{1}$, Egbert F. Knol ${ }^{3}$, \\ Rudie E. Koopmanschap ${ }^{2}$, Bas Kemp ${ }^{2}$ \& Nicoline M. Soede ${ }^{2}$ \\ ${ }^{1}$ Human and Animal Physiology, Wageningen University and Research, The Netherlands \\ ${ }^{2}$ Adaptation Physiology Group, Wageningen University and Research, The Netherlands \\ ${ }^{3}$ Topigs Norsvin Research Center B. V., Beuningen, The Netherlands
}

Published in Animal 2019 13(3): 554-563

DOI:10.1017/S1751731118001684 



\begin{abstract}
In this study we aimed to identify possible causes of within-litter variation in piglet birth weight (birth weight variation) by studying follicular development of sows at weaning in relation to their estimated breeding value (EBV) for birth weight variation. Twenty-nine multiparous sows (parity 3 to 5 ) were selected on their EBV for birth weight variation (SD in grams; High-EBV: $15.8 \pm 1.6, \mathrm{~N}=14$ and Low-EBV: $-24.7 \pm 1.5, \mathrm{~N}=15)$. The two groups of sows had similar litter sizes (15.7 vs. 16.9). Within 24 hours after parturition, piglets were cross fostered to ensure 13 suckling piglets per sow. Sows weaned $12.8 \pm 1.0$ and $12.7 \pm 1.0$ piglets, respectively, at day $26.1 \pm 0.2$ of lactation. Blood and ovaries were collected within two hours after weaning. The right ovary was immediately frozen to assess average follicle size and percentage healthy follicles of the 15 largest follicles. The left ovary was used to assess the percentage morphologically healthy cumulus-oocyte complexes (COCs) of the 15 largest follicles. To assess the metabolic state of the sows, body condition and the circulating metabolic markers insulin, insulin-like growth factor 1 , non-esterified fatty acid, creatinine, leptin, urea and fibroblast growth factor 21 were analysed at weaning. No significant differences were found in any of the measured follicular or metabolic parameters between High-EBV and Low-EBV. A higher weight loss during lactation was related to a lower percentage healthy $\operatorname{COCs}(\beta=-0.65, p=0.02)$. Serum creatinine, a marker for protein breakdown, was negatively related to average follicle size $(\beta=-0.60, p=0.05)$. Backfat loss during lactation was related to a higher backfat thickness at parturition and to a higher average follicle size $(\beta=0.36, p<0.001)$ at weaning. In conclusion, we hypothesise that modern hybrid sows with more backfat at the start of lactation are able to mobilise more energy from backfat during lactation and could thereby spare protein reserves to support follicular development.
\end{abstract}

Keywords: sows, litter uniformity, reproduction, lactation, metabolism 


\section{Introduction}

Over the last decades, pigs have been genetically selected to produce larger litters. Sows with larger litter sizes usually have lower average piglet birth weights (Tuchscherer et al., 2000) and higher birth weight variation (Tuchscherer et al., 2000; Milligan et al., 2002; Wientjes et al., 2012) which are related to higher piglet mortality during subsequent lactation (Milligan et al., 2002). Lower piglet birth weights and higher birth weight variation therefore strongly impact pig welfare and profitability (Fix et al., 2010). The cause and underlying mechanism of highly variable piglet birth weights and consequently higher birth weight variation are not completely clear. One factor that has been identified to increase birth weight variation is a negative energy balance (NEB) during lactation (Wientjes et al., 2013). This NEB negatively influences, during and after lactation, the development of follicles that will give rise to the next litter (reviewed by: Prunier and Quesnel, 2000). Therefore, we hypothesise that decreased piglet birth weights and increased variation in birth weight could result from impaired and more variable follicular development during the previous lactation.

Evidence for this hypothesis comes from studies in which the pre-mating metabolic state of sows affects embryonic development and uniformity (e.g. Ferguson et al., 2006; Patterson et al., 2011) and subsequent piglet birth weights and uniformity (van den Brand et al., 2006). In addition, a study by Wientjes et al. (2013) showed that body condition loss during lactation was related to increased variation in birth weight of the next litter. The influence of the metabolic state during lactation on variation in birth weight of the next litter may be explained by metabolic influences on follicular development during lactation. For instance, feed restriction during lactation has been shown to result in a decreased $\mathrm{LH}$ pulse frequency during lactation and around weaning (Quesnel et al., 1998a; van den Brand et al., 2000), a smaller follicle size at weaning and 48 hours later (Quesnel et al., 1998a) and decreased oocyte maturation rates when isolated 38 hours before the anticipated onset of oestrus (Zak et al., 1997). Furthermore, follicular heterogeneity has been related to embryonic heterogeneity (Pope et al., 1988 and 1989; Xie et al., 1990) which, in turn, is an important determinant of variation in birth weights (Van der Lende et al., 1990).

These observations together indicate the importance of a more detailed analysis of follicular development during lactation in order to identify possible causes for variation in birth weight. Therefore, the aim of the current study is to identify possible causes of variation in birth weight by studying follicular development and oocyte quality of sows directly after weaning. To investigate this, we compared sows with a high vs. low estimated breeding value (EBV) for variation in birth weight. In addition, the metabolic state of the sows is monitored to assess phenotypic relations between the metabolic state and follicular development at weaning. 


\section{Material and methods}

Animals

The experiment was approved by the Animal Care and Use Committee of Wageningen University (DEC2016036) and performed according to national and EU guidelines. A total of 30 multiparous Dutch Landrace sows (parity 3 to 5; Topigs Norsvin, Vught, the Netherlands) housed at a local farm were selected based on EBV for variation in birth weight. Breeders can produce an EBV for each individual sow and it is regarded as the best estimate of the genetic potential for heritable traits such as variation in birth weight. The EBV is expressed as the standard deviation of the piglet birth weights within one litter in grams and is calculated based on genetic background and previous performance. Average EBV of all parity 3 to 5 animals on the farm was $-6.3 \pm 17.1$ ( -41.9 to 28.0$)$. A total of 15 sows were selected with a high EBV variation in birth weight (High-EBV; $15.8 \pm 1.6 ; 5.4$ to 28.0 ) and 15 sows were selected with a low EBV (Low-EBV; $-24.7 \pm 1.5 ;-14.9$ to -31.1 ). Average parity was $3.7 \pm 0.3$ for High-EBV sows and 3.9 \pm 0.2 for Low-EBV sows. The sows were fed a standard lactation diet (ca. $12.5 \mathrm{MJ} \mathrm{NE} / \mathrm{kg}$, 154 g/kg CP, 9.3 g/kg lysine; Lacto Excellent, Agrifirm, Apeldoorn, The Netherlands) and feed intake was assessed daily. Within 24 hours after parturition, piglets were cross fostered to ensure 13 suckling piglets per sow. The sows had a lactation period of $26.1 \pm 0.2$ ( 25 to 27 ) days and the experiment took place over a period of 10 weeks. Each week, 1 or 2 sows of each group (High-EBV and Low-EBV; so, a total of 2 to 4 sows per week) entered the experiment. The sows were slaughtered at the slaughterhouse by stunning and exsanguination within 2 hours after weaning.

\section{Body weight and backfat thickness}

The sows were weighed approximately 1 week before parturition and immediately after weaning. Weight after parturition was estimated according to Bergsma et al. (2009) by correcting the body weight of the sows as measured 1 week before parturition for weight of the foetuses, placenta and intra-uterine fluid. Backfat thickness was measured $6.5 \mathrm{~cm}$ from the midline over the last rib both on the left and the right side using A-mode ultrasonography (Renco Lean-Meater, Renco Corporation, Golden Valley, MN, USA) 1 week before parturition, within 12 hours after parturition and immediately after weaning. Weight loss during lactation was calculated by subtracting body weight at weaning from the estimated body weight at parturition. A correction according to Bergsma et al. (2009) was used for water content of mammary glands to prevent underestimation of body weight loss, since mammary glands contain more water at the end of lactation compared to the start of lactation. Body weight loss was expressed as a percentage of bodyweight at parturition. Piglets were weighed within 24 hours after parturition and at weaning (26.1 \pm 0.2 days postpartum). Litter growth during lactation, as a measure for sow milk production, was calculated and corrected for mortality weight of the piglets that died during lactation, according to Bergsma et al. (2009). 


\section{Collection of ovaries and blood samples}

The left ovary was stored in a thermo-container and covered with the uterus to keep the ovaries at a temperature above $30^{\circ} \mathrm{C}$ for subsequent follicle aspiration. The right ovary was cut in 2 halves, immediately frozen in liquid nitrogen and stored at $-80^{\circ} \mathrm{C}$ until further analysis. Blood was collected from the jugular vein at slaughter in $9 \mathrm{ml}$ serum clot activator collection tubes and in $9 \mathrm{ml}$ EDTA coated tubes (Greiner Bio-One, Monroe, NC, USA), to obtain serum and plasma samples, respectively. The serum collection tubes and the EDTA coated tubes were stored on ice and transported to the laboratory. In the lab, the EDTA tubes were immediately centrifuged at $500 \mathrm{xg}$ for $10 \mathrm{~min}$ at $4^{\circ} \mathrm{C}$ to collect plasma. The serum tubes were first incubated overnight at $4{ }^{\circ} \mathrm{C}$ and subsequently centrifuged to collect serum. Both plasma and serum samples were stored in $-20^{\circ} \mathrm{C}$ until further analysis.

\section{Measurements}

Left ovary (fresh) The left ovary was, after transportation to the lab, placed in phosphate buffered saline $\mathrm{pH} 7.4$ (PBS) in a water bath at $37^{\circ} \mathrm{C}$. Within 5 hours after slaughter, the ovaries were used for follicle aspiration. The 15 largest follicles were aspirated as these are assumed to represent approximately half of the ovulatory follicle pool, as ovulation rates in modern sows are around 25-30 (da Silva et al., 2016). The contents were collected in a tube and allowed to settle for $5 \mathrm{~min}$. The supernatant was removed and centrifuged at $1900 \mathrm{xg}$ at $4{ }^{\circ} \mathrm{C}$ for $30 \mathrm{~min}$ to collect the follicular fluid and assess the amount of follicular fluid. The recovered cumulus-oocyte complexes (COCS) were morphologically classified under a dissection microscope as normal (intact cumulus and normal-shaped oocyte) or atretic (degraded cumulus or degenerated oocyte), according to Alvarez et al. (2009).

Right ovary (frozen) The 2 halves of the right ovary were used to measure follicle size. Of each half of the ovary, 3 cutting planes were made in a cryostat in order to study (almost) all follicles on the surface of the ovary. Of each of these cutting planes, photographs were taken; the ovaries were held against a ruler to measure the size of the follicles. Follicle size was determined as the largest macroscopically visible diameter of the follicle. To determine if the follicles were healthy or atretic, cryo-sections of the right ovary were made and immunohistochemical staining for the presence of cleaved-Caspase 3 was performed (Supplemental Fig. S2.1), a marker for cells in apoptosis similar to Slot et al. (2006). In short, cryo-sections were mounted on superfrost plus glass slides (Menzel- Gläser, Braunschweig, Germany). Sections were fixed in $4 \%$ buffered formalin for $10 \mathrm{~min}$, washed in $\mathrm{H}_{2} \mathrm{O}$, microwaved for $3 \times 5 \mathrm{~min}$ in $0.1 \mathrm{M}$ sodium citrate buffer ( $\mathrm{pH} 6)$ for epitope antigen retrieval, cooled down to room temperature and subsequently rinsed PBS pH 7.4. Endogenous peroxidase activity was blocked with $3 \%(\mathrm{v} / \mathrm{v})$ hydrogen peroxide in methanol solution for 30 min and aldehyde residues were blocked with $0.3 \%$ glycine in PBS for $10 \mathrm{~min}$. After rinsing with PBS, sections were pre-incubated with $5 \%$ (wt/v) normal goat serum in PBS for 60 min at room temperature. Subsequently, the sections were incubated overnight at $4^{\circ} \mathrm{C}$ in a humid 
chamber with primary polyclonal rabbit anti-cleaved-Caspase 3 antibody (9661S, Cell Signalling Technology, Danvers, MA, USA) diluted 1:1000 (v/v) in PBS-BSA-c (Aurion, Wageningen, The Netherlands). Next, sections were rinsed with PBS and treated with a secondary biotin labelled goat-anti-rabbit antibody (Vector Laboratories, Burlingame, CA, USA) diluted 1:400 (v/v) in PBS-BSAc for 1 hour at room temperature. After a wash with PBS and incubation with avidin-biotin complex $(A B C)$ diluted 1:1500 (v/v) in PBS-BSAc (Vector stain kit Elite, Vector Laboratories) for $60 \mathrm{~min}$ at room temperature, sections were rinsed with PBS and bound antibody was visualized using the Immpact DAB kit (stock solution diluted 1:400 (v/v); Vector Laboratories). Sections were briefly counterstained with Mayer's haematoxylin (Klinipath, Duiven, The Netherlands), visualised using light microscopy (Axioskop 2, Carl Zeiss Microscopy, Thornwood, NY, US) and imaged using imaging software (Axiovision 4.8, Carl Zeiss Microscopy).

\section{Assay procedures}

All assay procedures were performed according to manufacturer's instructions, unless stated otherwise. All analyses were performed in duplo and only samples with an intra-assay CV $\leq$ $15 \%$ were included. Plasma insulin and leptin concentrations were measured using a radioimmunoassay kit (Porcine Insulin $\mathrm{PI}-12 \mathrm{~K}$ and Multi-Species Leptin $\mathrm{XL}-85 \mathrm{~K}$, respectively, EMD Millipore corporation, Billerica, MA, US), fibroblast growth factor 21 (FGF21) was measured using an ELISA kit (Abbexa, Cambridge, UK) and plasma urea and creatinine were measured using an enzymatic colorimetric test (Urea liquicolor, Human Gesselschaft fur Biochemica und Diganostica $\mathrm{mbH}$, Wiesbaden, Germany and Creatinine PAP FS, DiaSys Diagnostic Systems GmbH, Holzheim, Germany, respectively). Plasma insulin-like growth factor 1 (IGF1) was measured with an immunoradiometric assay according to the manufacturer's protocol (A15729, Beckman Coulter, Woerden, The Netherlands) supplemented with additional acid-ethanol extraction $(87.5 \% \mathrm{v} / \mathrm{v}$ EtOH and $2.9 \% \mathrm{v} / \mathrm{v} 12 \mathrm{~N}$ $\mathrm{HCl}$ ). For serum non-esterified fatty acid (NEFA) analysis, a calorimetric detection method was used (NEFA-HR(2) kit, Wako Chemicals, Neuss, Germany). Different from the manufacturer's protocol, we added $5 \mu \mathrm{l}$ serum to the plate and $100 \mu \mathrm{l}$ of reagent 1 was added to the wells and incubated for $10 \mathrm{~min}$ at $37^{\circ} \mathrm{C}$. Subsequently, $50 \mu$ of reagent 2 was added and another incubation step of $10 \mathrm{~min}$ at $37^{\circ} \mathrm{C}$ followed.

\section{Statistical analyses}

One of the animals of the High-EBV group ovulated during lactation and was excluded from further analyses. Body weight of one animal was not recorded before farrowing and 2 FGF21 values were removed because the $\mathrm{CV}$ values were $\geq 15 \%$. Distributions of the means and residuals were examined to verify model assumptions of normality and homogeneity of variance. The presence of outliers was tested by calculating the studentized residuals using proc REG and 2 outliers ( 1 NEFA and 1 urea value) were removed from further analyses. Follicular and metabolic differences between EBV classes (High-EBV, N=14 and Low-EBV, 
$\mathrm{N}=15$ ), follicle size classes (FS: large: $>5.1 \mathrm{~mm}$ average follicle size of the 15 largest follicles of the right ovary $(\mathrm{N}=14)$ and small: $<5.0 \mathrm{~mm}$ average follicle size $(\mathrm{N}=15))$, variation in follicle size classes (VARFS: large: $>0.09 \mathrm{~mm}$ SD in follicle size of the 15 largest follicles of the right ovary $(\mathrm{N}=15)$ and small: $<0.09 \mathrm{~mm}(\mathrm{~N}=14))$ were analysed using proc GLM in SAS 9.4 (Cary, $\mathrm{NC})$ in models that also contained the factor PAR (PAR3 (parity 3, N=14) and PAR4+5 (parity 4 and 5, $\mathrm{N}=15$ )) and the interaction with PAR. Interactions were excluded from the models when not significant. All values are presented as LS means. Additionally, relations between metabolic parameters and between metabolic and follicular parameters were estimated using the model: $Y_{i j k}=\mu+E B V+P A R+\beta X_{i j k}+E B V * P A R+\beta X^{*} P A R+\varepsilon_{i j k}$, where $Y_{i j k}$ is the dependent variable and either a metabolic or follicular parameter, $\beta$ is the regression coefficient and $X_{i j k}$ is one of the metabolic parameters. The interactions were excluded from the models when not significant.

\section{Results}

\section{Follicular parameters}

Right ovary (frozen): Average follicle size of the 15 largest follicles was $5.04 \pm 0.74 \mathrm{~mm}$ while average follicle size of the 10 largest healthy follicles was $5.11 \pm 0.82 \mathrm{~mm}$. Of the 15 largest follicles, $67.1 \pm 17.3 \%$ was classified as healthy based on cleaved-Caspase 3 staining. Left ovary (fresh): $72.1 \pm 21.1 \%$ of the cumulus-oocytes complexes (COCs) isolated from the 15 largest follicles was classified as healthy. The total amount of follicular fluid of the 15 largest follicles was $369 \pm 153 \mu$ l.

A

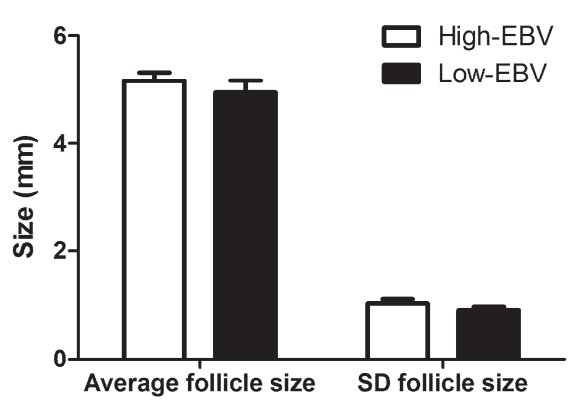

B

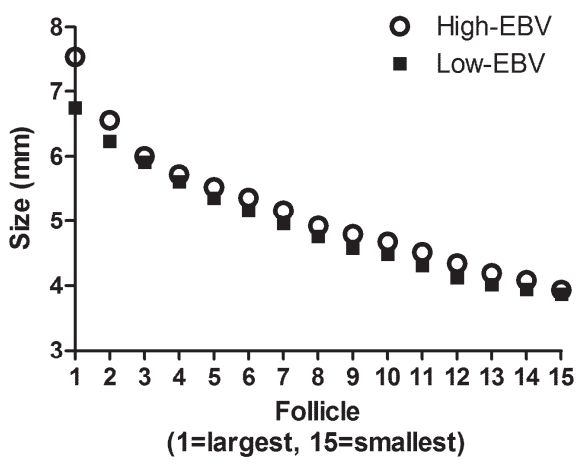

Figure 2.1 - (A) Average follicle size and variation (SD) in follicle size of the 15 largest follicles of the right ovary for estimated breeding value (EBV) class for within-litter variation in piglet birth weight (High-EBV ( $\mathrm{N}=14)$ and Low-EBV $(\mathrm{N}=15)$ ) (B) Average size $(\mathrm{cm})$ of the 15 largest follicles (1=largest, $15=$ smallest) of the right ovary for High-EBV and Low-EBV sows.

\section{EBV class for within-litter variation in piglet birth weight}

High-EBV sows had an average EBV for variation in birth weight of $15.8 \pm 1.6$ and Low-EBV had an average EBV of $-24.7 \pm 1.5$ ( $p<0.001$ ). High-EBV and Low-EBV sows did not differ in body 
condition or any of the measured metabolic parameters nor did they differ in any of the piglet parameters (average birth weight, variation in birth weight (SD), litter growth during lactation; Table 2.1). In addition, follicular parameters at weaning did not differ between sows with HighEBV and Low-EBV; neither average follicle size or variation in follicle size of the 15 largest follicles (Fig. 2.1), nor percentage healthy COCs or percentage healthy follicles (Fig. 2.2; all Supplemental Table S2.1). Interactions between EBV class and PAR were never significant.

Table 2.1 - Effects of estimated breeding value classes for within-litter variation in piglet birth weight (EBV; High ( $\mathrm{N}=14)$ vs. Low ( $\mathrm{N}=15))$ and parity classes (PAR; $3(\mathrm{~N}=14)$ vs. $4+5(\mathrm{~N}=15))$ on gestation and lactation parameters, body condition and metabolic parameters at weaning in sows. All values are presented as LS means.

\begin{tabular}{|c|c|c|c|c|c|c|c|}
\hline \multirow[b]{2}{*}{ Parameter } & \multicolumn{2}{|c|}{ EBV } & \multicolumn{2}{|c|}{ PAR } & \multirow[t]{2}{*}{ RMSE } & \multicolumn{2}{|c|}{ P-values ${ }^{1}$} \\
\hline & High & Low & 3 & $4+5$ & & EBV & PAR \\
\hline EBV LVR (g) & 15.8 & -24.7 & -6.3 & -2.6 & 5.7 & $<0.001$ & 0.11 \\
\hline \multicolumn{8}{|l|}{ Gestation + parturition } \\
\hline Gestation (days) & 115.2 & 114.9 & 114.8 & 115.3 & 1.1 & 0.51 & 0.27 \\
\hline Total number born & 15.7 & 16.9 & 17.2 & 15.4 & 3.6 & 0.43 & 0.19 \\
\hline Average piglet birth weight & 1476 & 1371 & 1390 & 1457 & 258 & 0.31 & 0.51 \\
\hline Variation $(\mathrm{SD})(\mathrm{g})$ & 267 & 291 & 313 & 275 & 69 & 0.84 & 0.18 \\
\hline Total litter weight start $(\mathrm{kg})$ & 19.7 & 18.0 & 18.6 & 19.2 & 3.0 & 0.17 & 0.63 \\
\hline \multicolumn{8}{|l|}{ Lactation } \\
\hline Lactation (days) & 26.1 & 26.2 & 26.3 & 26.0 & 0.89 & 0.80 & 0.89 \\
\hline Total litter weight weaning & 91.1 & 90.2 & 87.5 & 93.8 & 9.9 & 0.83 & 0.12 \\
\hline Total litter growth $(\mathrm{kg})$ & 71.8 & 72.5 & 69.4 & 75.0 & 9.1 & 0.84 & 0.13 \\
\hline $\mathrm{N}$ of piglets weaned & 12.8 & 12.7 & 12.7 & 12.7 & 0.6 & 0.87 & 0.87 \\
\hline Feed intake sow (kg/day) & 6.0 & 5.9 & 6.0 & 5.9 & 0.5 & 0.45 & 0.71 \\
\hline \multicolumn{8}{|l|}{ Body condition } \\
\hline Weight parturition (kg) & 248 & 248 & 248 & 251 & 20 & 0.91 & 0.47 \\
\hline Weight weaning $(\mathrm{kg})$ & 232 & 232 & 225 & 239 & 15 & 0.99 & 0.02 \\
\hline Weight loss lactation (\%) & 10.6 & 10.3 & 11.1 & 9.8 & 6.3 & 0.91 & 0.60 \\
\hline Backfat parturition (mm) & 17.0 & 17.1 & 16.6 & 17.5 & 2.2 & 0.91 & 0.30 \\
\hline Backfat weaning $(\mathrm{mm})$ & 13.9 & 14.4 & 14.3 & 13.9 & 1.9 & 0.48 & 0.61 \\
\hline Backfat loss lactation (mm) & 3.1 & 2.7 & $2.3^{\mathrm{a}}$ & $3.5^{\mathrm{b}}$ & 1.3 & 0.42 & 0.02 \\
\hline \multicolumn{8}{|l|}{ Metabolic parameters } \\
\hline Insulin (uU/ml) & 11.8 & 11.9 & 14.1 & 9.6 & 7.7 & 0.96 & 0.15 \\
\hline IGF1 (ng/ml) & 154 & 136 & 139 & 151 & 63 & 0.47 & 0.64 \\
\hline FGF21 (pg/ml) & 5813 & 8861 & 7978 & 6697 & 3143 & 0.06 & 0.40 \\
\hline Urea (mmol/l) & 4.46 & 4.33 & 4.23 & 4.56 & 1.00 & 0.74 & 0.43 \\
\hline Creatinine (mg/dl) & 2.38 & 2.31 & $2.15^{\mathrm{a}}$ & $2.54^{b}$ & 0.48 & 0.69 & 0.05 \\
\hline NEFA (mmol/L) & 0.98 & 0.97 & 1.01 & 0.94 & 0.45 & 0.92 & 0.73 \\
\hline Leptin (ng/ml) & 13.6 & 13.0 & 11.4 & 10.5 & 2.3 & 0.67 & 0.33 \\
\hline
\end{tabular}

$\overline{\mathrm{EBV}}=$ estimated breeding value, $\mathrm{LVR}=$ within-litter variation in piglet birth weight (standard deviation), NEFA $=$ non-esterified fatty acid, IGF1 = insulin-like growth factor 1, FGF = fibroblast growth factor. ${ }^{1}$ Interactions between EBV and PAR were never significant. 


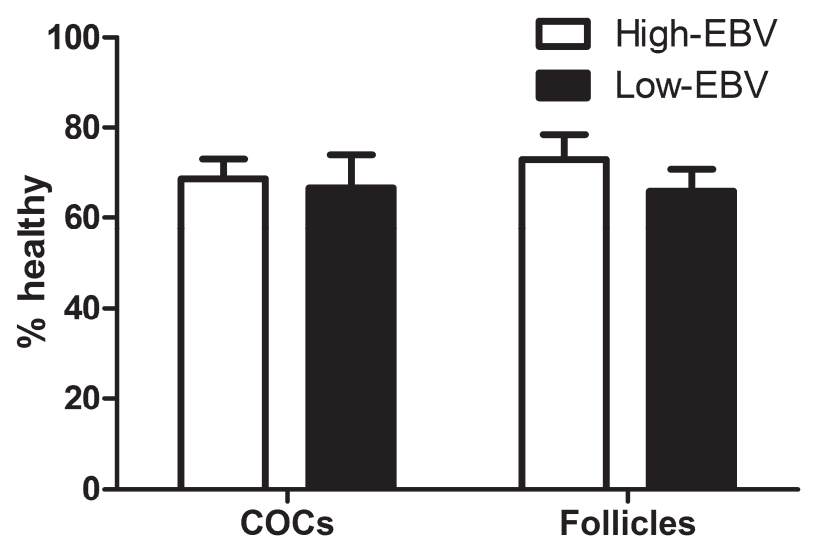

Figure 2.2 - Percentage healthy cumulus-oocyte complexes $(\mathrm{COC})$ of the left ovary and percentage healthy follicles of the right ovary for estimated breeding value (EBV) class for within-litter variation in piglet birth weight (High-EBV $(\mathrm{N}=14)$ and Low-EBV $(\mathrm{N}=15)$ ) in sows. The COCs were morphologically classified as healthy or atretic according to Alvarez et al. (2009) and follicles were classified as healthy or atretic using the cleaved-Caspase 3 staining as a marker for cells in apoptosis.

\section{Follicle size class (FS)}

Large-FS sows (average follicle size of the 15 largest follicles $>5.1 \mathrm{~mm}$ ) had a higher backfat thickness at parturition (17.9 vs. $16.1 ; p=0.02$ ), higher backfat loss during lactation (4.0 vs. 2.6; $\mathrm{P}<0.01)$ and lower creatinine levels at weaning $(2.13$ vs. $2.52 ; \mathrm{p}=0.03)$ compared to Small-FS $(<5 \mathrm{~mm})$ sows (Table 2.2). Large-FS sows did not differ in any of the follicular parameters except for follicle size (Supplemental Table S2.2). Interactions between FS and PAR were only significant for bodyweight at parturition (Small-FS*PAR3 $=228$, SmallFS*PAR4 +5 = 259, Large-FS*PAR3 $=252$, Large-FS*PAR4+5 = 239; $p<0.01$ ) and plasma insulin levels at weaning (Small-FS*PAR3 $=8.5$, Small-FS*PAR4 $+5=11.3$, Large-FS*PAR3 $=17.2$, LargeFS*PAR4+5 =7.3; $p=0.03$ ).

\section{Variation in follicle size class (VARFS)}

Large-VARFS sows (variation (SD) in follicle size of the 15 largest follicles $>0.09 \mathrm{~mm}$ ) vs. SmallVARFS sows $(<0.09 \mathrm{~mm})$ did not differ in any of the metabolic (Table 2.3) or follicular parameters, except for variation in follicle size (Supplemental Table S2.3). Interactions between VARFS and PAR were only significant for urea levels at weaning (Small-VARFS*PAR3 $=4.1$, Small-VARFS*PAR4+5 $=5.1$, Large-VARFS*PAR3 $=4.3$, Large-VARFS*PAR4+5 $=3.7 ; p=$ $0.04)$.

\section{Parity class}

PAR4 +5 sows had a higher body weight at weaning ( 239 vs. $225 ; p=0.02$ ) and higher creatinine levels at weaning ( 2.51 vs. $2.15 ; p=0.05$ ) and lost more backfat during lactation (3.5 vs. $2.3 ; p$ $=0.02$ ) compared to PAR3 sows (Table 2.1). Sows with a different parity class did not differ in any of the measured follicular parameters (Supplemental Table S2.1). 
Table 2.2 - Effects of average sow follicle size classes (FS; Small $<5.0 \mathrm{~mm}(\mathrm{~N}=15)$ vs. Large $>5.1 \mathrm{~mm}$ $(\mathrm{N}=14))$ and parity classes (PAR; $3(\mathrm{~N}=14)$ vs. $4+5(\mathrm{~N}=15))$ on gestation and lactation parameters, body condition and metabolic parameters at weaning in sows. All values are presented as LS means.

\begin{tabular}{|c|c|c|c|c|c|c|c|}
\hline \multirow{3}{*}{ Parameter } & \multirow{2}{*}{\multicolumn{2}{|c|}{ FS }} & \multirow{2}{*}{\multicolumn{2}{|c|}{ PAR }} & \multirow{3}{*}{ RMSE } & \multirow{2}{*}{\multicolumn{2}{|c|}{ P-values }} \\
\hline & & & & & & & \\
\hline & Small & Large & 3 & $4+5$ & & FS & PAR \\
\hline EBV LVR (g) & -9.6 & -0.3 & -1.8 & -8.4 & 20.5 & 0.24 & 0.41 \\
\hline \multicolumn{8}{|l|}{ Gestation + parturition } \\
\hline Gestation (days) & 114.8 & 115.3 & 114.8 & 115.3 & 1.1 & 0.22 & 0.22 \\
\hline Total number born & 15.4 & 17.2 & 16.8 & 15.7 & 3.5 & 0.20 & 0.42 \\
\hline Average piglet birth weight & 1473 & 1375 & 1418 & 1430 & 258 & 0.33 & 0.91 \\
\hline Variation (SD) (g) & 293 & 295 & 313 & 275 & 69 & 0.93 & 0.16 \\
\hline Total litter weight start (kg) & 19.3 & 18.5 & 18.9 & 18.5 & 3.1 & 0.51 & 0.91 \\
\hline \multicolumn{8}{|l|}{ Lactation } \\
\hline Lactation (days) & 26.0 & 26.2 & 26.3 & 26.0 & 0.9 & 0.54 & 0.50 \\
\hline Total litter weight weaning & 90.0 & 91.3 & 87.5 & 93.8 & 9.9 & 0.73 & 0.11 \\
\hline Total litter growth (kg) & 70.8 & 73.4 & 68.9 & 75.3 & 9.0 & 0.46 & 0.08 \\
\hline $\mathrm{N}$ of piglets weaned & 12.8 & 12.7 & 12.8 & 12.7 & 0.6 & 0.76 & 0.76 \\
\hline Feed intake sow (kg/day) & 5.8 & 6.0 & 6.0 & 5.9 & 0.5 & 0.34 & 0.70 \\
\hline \multicolumn{8}{|l|}{ Body condition } \\
\hline Weight parturition $(\mathrm{kg})^{1}$ & 244 & 246 & 240 & 249 & 20 & 0.76 & 0.20 \\
\hline Weight weaning (kg) & 234 & 230 & 225 & 239 & 15 & 0.53 & 0.03 \\
\hline Weight loss lactation (\%) & 10.6 & 10.3 & 11.2 & 9.7 & 6.3 & 0.68 & 0.61 \\
\hline Backfat parturition (mm) & 16.1 & 17.9 & 16.3 & 17.7 & 2.0 & 0.02 & 0.08 \\
\hline Backfat weaning (mm) & 13.9 & 14.3 & 13.9 & 14.3 & 1.9 & 0.61 & 0.86 \\
\hline Backfat loss lactation (mm) & 2.6 & 4.0 & 2.7 & 3.9 & 1.1 & $<0.01$ & $<0.01$ \\
\hline \multicolumn{8}{|l|}{ Metabolic parameters } \\
\hline Insulin $(\mathrm{uU} / \mathrm{ml})^{2}$ & 9.9 & 12.3 & 12.8 & 9.3 & 7.6 & 0.40 & 0.21 \\
\hline IGF1 (ng/ml) & 153 & 138 & 144 & 147 & 63 & 0.56 & 0.88 \\
\hline FGF21 (pg/ml) & 7296 & 7758 & 7303 & 7751 & 3420 & 0.75 & 0.76 \\
\hline Urea $(\mathrm{mmol} / \mathrm{l})$ & 4.6 & 4.3 & 4.3 & 4.5 & 1.0 & 0.49 & 0.53 \\
\hline Creatinine (mg/dl) & 2.52 & 2.13 & 2.22 & 2.43 & 044 & 0.03 & 0.21 \\
\hline NEFA (mmol/L) & 0.91 & 1.02 & 0.98 & 0.95 & 0.45 & 0.54 & 0.82 \\
\hline Leptin (ng/ml) & 10.7 & 11.2 & 11.4 & 10.5 & 2.28 & 0.61 & 0.31 \\
\hline
\end{tabular}

$\mathrm{EBV}=$ estimated breeding value, $\mathrm{LVR}=$ within-litter variation in piglet birth weight (standard deviation), NEFA $=$ non-esterified fatty acid, IGF1 = insulin-like growth factor 1, FGF = fibroblast growth factor. ${ }^{1} \mathrm{LS}$ means estimates for the interaction FS*PAR $(p<0.01)$ : Small-FS*PAR3 $=228$, Small-FS*PAR4 $+5=259$, Large-FS*PAR3 $=252$, LargeFS*PAR4 $+5=239$. ${ }^{2}$ LS means estimates for the interaction FS*PAR $(p=0.03)$ : Small-FS*PAR3 $=8.5$, SmallFS*PAR4+5 $=11.3$, Large-FS*PAR3 $=17.2$, Large-FS*PAR4 $+5=7.3$.

Weight loss during lactation

More body weight loss during lactation was related to lower plasma IGF1 levels at weaning ( $\beta$ $=-6.43 \mathrm{ng} / \mathrm{ml}$ per $\%, \mathrm{p}<0.01)$, higher serum creatinine levels at weaning $(\beta=0.01 \mathrm{mg} / \mathrm{dl}$ per $\%, p=0.05)$ and to a smaller percentage healthy $\operatorname{COCs}(\beta=-0.65 \% / \%, p=0.02$; all Fig. 2.3). Furthermore, higher IGF1 levels tended to be related to a higher percentage healthy COCs ( $\beta$ $=0.001 \%$ per $\mathrm{ng} / \mathrm{ml}, \mathrm{p}=0.10$ ), while higher creatinine levels were related to a smaller average 
follicle size ( $\beta=-0.60 \mathrm{~mm}$ per $\mathrm{mg} / \mathrm{dl}, \mathrm{p}=0.05$; Fig 2.3 ) and serum urea levels were not related to any of the measured metabolic or follicular parameters.
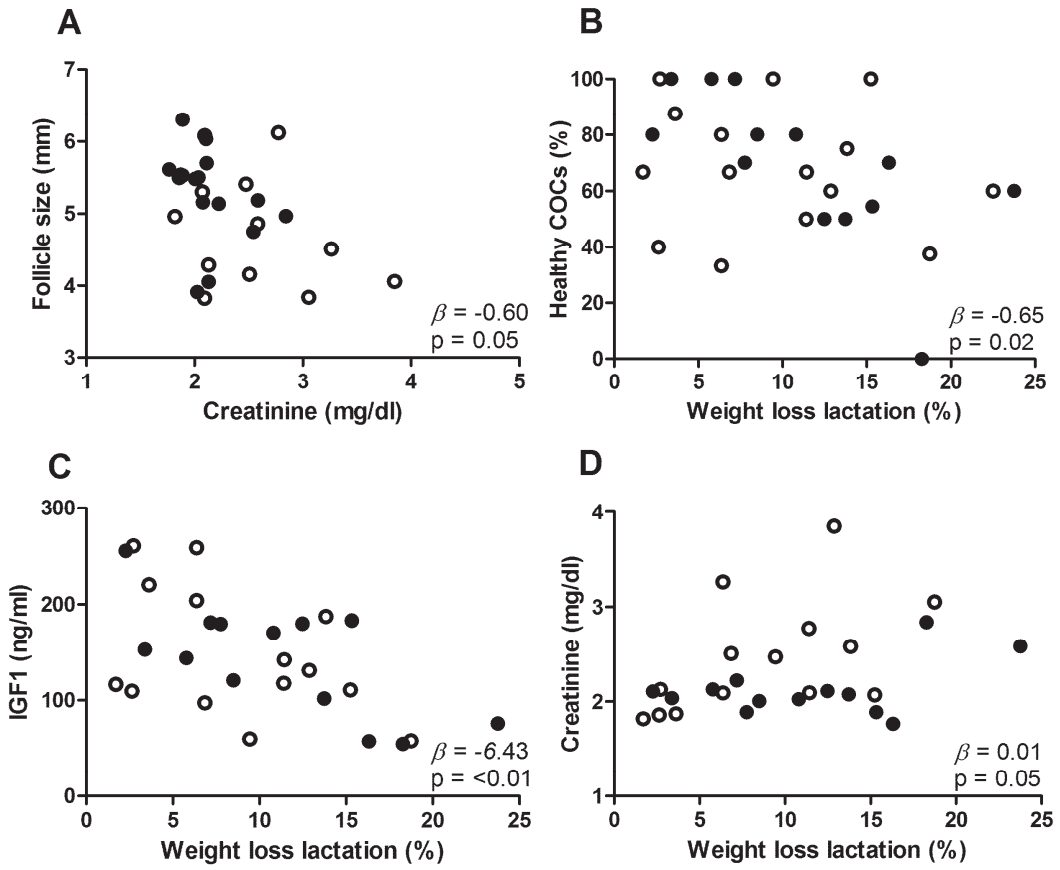

Figure 2.3 - Regression equations $(\beta)$ for the relations between $(A)$ creatinine levels at weaning $(\mathrm{mg} / \mathrm{dl})$ and follicle size of the 15 largest follicles at weaning and weight loss during lactation (\%) and (B) percentage healthy cumulus-oocyte complexes (COCs), (C) insulin-like growth factor 1 (IGF1) (ng/ml) levels at weaning and (D) creatinine levels $(\mathrm{mg} / \mathrm{dl})$ at weaning in sows. The COCs were morphologically classified as healthy or atretic according to (Alvarez et al., 2009). No interactions with parity class (PAR) have been found.

\section{Backfat loss during lactation}

A higher backfat thickness at parturition was related to a higher backfat loss during lactation ( $\beta=0.92 \mathrm{~mm} / \mathrm{mm}, p<0.01$, Supplemental Fig. S2.2). In addition, a higher backfat loss during lactation was related to higher serum NEFA levels at weaning $(\beta=0.15 \mathrm{mmol} / \mathrm{L}$ per $\mathrm{mm}$, $p=0.03$; Fig. 2.4) and lower creatinine levels $(\beta=-0.14 \mathrm{mg} / \mathrm{dl}$ per $\mathrm{mm}, \mathrm{p}=0.05$; Fig. 2.4). A higher backfat thickness at parturition and a higher backfat loss during lactation were both related to a higher average follicle size of the 15 largest follicles at weaning $(\beta=0.19 \mathrm{~mm} / \mathrm{mm}$, $p=0.01$ and $\beta=0.36 \mathrm{~mm} / \mathrm{mm}, \mathrm{p}<0.001 ;$ Fig. 2.4, respectively). A higher backfat loss during lactation was also related to a higher average follicle size of the 10 largest healthy follicles ( $\beta=0.38 \mathrm{~mm} / \mathrm{mm}, p=0.01$; Fig. 2.4). 
Table 2.3 - Effects of average sow variation (SD) in follicle size classes (VARFS; Small $<0.09 \mathrm{~mm}(\mathrm{~N}=14$ ) vs. Large $>0.09 \mathrm{~mm}(\mathrm{~N}=15))$ and parity classes (PAR; $3(\mathrm{~N}=14)$ vs. $4+5(\mathrm{~N}=15))$ on gestation and lactation parameters, body condition and metabolic parameters at weaning in sows. All values are presented as LS means.

\begin{tabular}{|c|c|c|c|c|c|c|c|}
\hline \multirow[b]{2}{*}{ Parameter } & \multicolumn{2}{|c|}{ VARFS } & \multicolumn{2}{|c|}{ PAR } & \multirow[t]{2}{*}{ RMSE } & \multicolumn{2}{|c|}{ P-values } \\
\hline & Small & Large & 3 & $4+5$ & & VARFS & PAR \\
\hline EBV LVR (g) & -1.7 & -7.8 & 0.4 & -9.9 & 20.9 & 0.45 & 0.21 \\
\hline \multicolumn{8}{|l|}{ Gestation + parturition } \\
\hline Gestation (days) & 114.9 & 115.2 & 114.8 & 115.3 & 1.1 & 0.58 & 0.30 \\
\hline Total number born & 16.1 & 16.5 & 17.0 & 15.6 & 3.6 & 0.74 & 0.31 \\
\hline Average piglet birth weight & 1409 & 1434 & 1401 & 1442 & 263 & 0.81 & 0.69 \\
\hline Variation (SD) (g) & 282 & 305 & 310 & 277 & 68 & 0.38 & 0.21 \\
\hline Total litter weight start (kg) & 18.8 & 18.9 & 18.8 & 18.9 & 3.1 & 0.92 & 0.94 \\
\hline \multicolumn{8}{|l|}{ Lactation } \\
\hline Lactation (days) & 26.0 & 26.2 & 26.3 & 26.0 & 0.9 & 0.54 & 0.50 \\
\hline Total litter weight weaning & 90.4 & 90.9 & 87.6 & 93.7 & 9.9 & 0.90 & 0.12 \\
\hline Total litter growth (kg) & 71.7 & 72.6 & 69.2 & 75.2 & 9.2 & 0.82 & 0.10 \\
\hline $\mathrm{N}$ of piglets weaned & 12.8 & 12.7 & 12.8 & 12.7 & 0.6 & 0.76 & 0.76 \\
\hline Feed intake sow (kg/day) & 5.9 & 5.9 & 6.0 & 5.8 & 0.5 & 0.97 & 0.55 \\
\hline \multicolumn{8}{|l|}{ Body condition } \\
\hline Weight parturition (kg) & 248 & 248 & 245 & 251 & 19.7 & 0.99 & 0.42 \\
\hline Weight weaning (kg) & 231 & 233 & 224 & 239 & 15 & 0.71 & 0.02 \\
\hline Weight loss lactation (\%) & 11.0 & 9.9 & 11.3 & 9.6 & 6.3 & 0.67 & 0.49 \\
\hline Backfat parturition (mm) & 16.4 & 17.6 & 16.4 & 17.6 & 2.1 & 0.14 & 0.14 \\
\hline Backfat weaning (mm) & 13.9 & 14.4 & 14.2 & 14.1 & 1.9 & 0.54 & 0.88 \\
\hline Backfat loss lactation (mm) & 3.2 & 3.5 & 2.9 & 3.8 & 1.3 & 0.54 & 0.08 \\
\hline \multicolumn{8}{|l|}{ Metabolic parameters } \\
\hline Insulin (uU/ml) & 12.08 & 11.7 & 14.1 & 9.6 & 7.7 & 0.89 & 0.14 \\
\hline IGF1 (ng/ml) & 152 & 139 & 144 & 147 & 63 & 0.59 & 0.88 \\
\hline FGF21 (pg/ml) & 7658 & 7468 & 7413 & 7712 & 3426 & 0.89 & 0.83 \\
\hline Urea $(\mathrm{mmol} / \mathrm{l})^{1}$ & 4.6 & 4.0 & 4.2 & 4.4 & 1.0 & 0.12 & 0.64 \\
\hline Creatinine (mg/dl) & 2.32 & 2.30 & 2.16 & 2.46 & 0.48 & 0.92 & 0.13 \\
\hline NEFA (mmol/L) & 1.12 & 0.86 & 1.06 & 0.92 & 0.43 & 0.15 & 0.43 \\
\hline Leptin (ng/ml) & 11.0 & 11.0 & 11.5 & 10.5 & 2.3 & 0.92 & 0.27 \\
\hline
\end{tabular}

$\overline{E B V}=$ estimated breeding value, $\mathrm{LVR}=$ within-litter variation in piglet birth weight (standard deviation), NEFA $=$ non-esterified fatty acid, IGF1 = insulin-like growth factor 1, FGF= fibroblast growth factor. ${ }^{1}$ LS means estimates for the interaction VARFS*PAR $(p=0.04)$ : Small-VARFS*PAR3 $=4.1$, Small-VARFS*PAR4 $+5=5.1$, LargeVARFS $*$ PAR3 $=4.3$, Large-VARFS $*$ PAR $4+5=3.7$. 

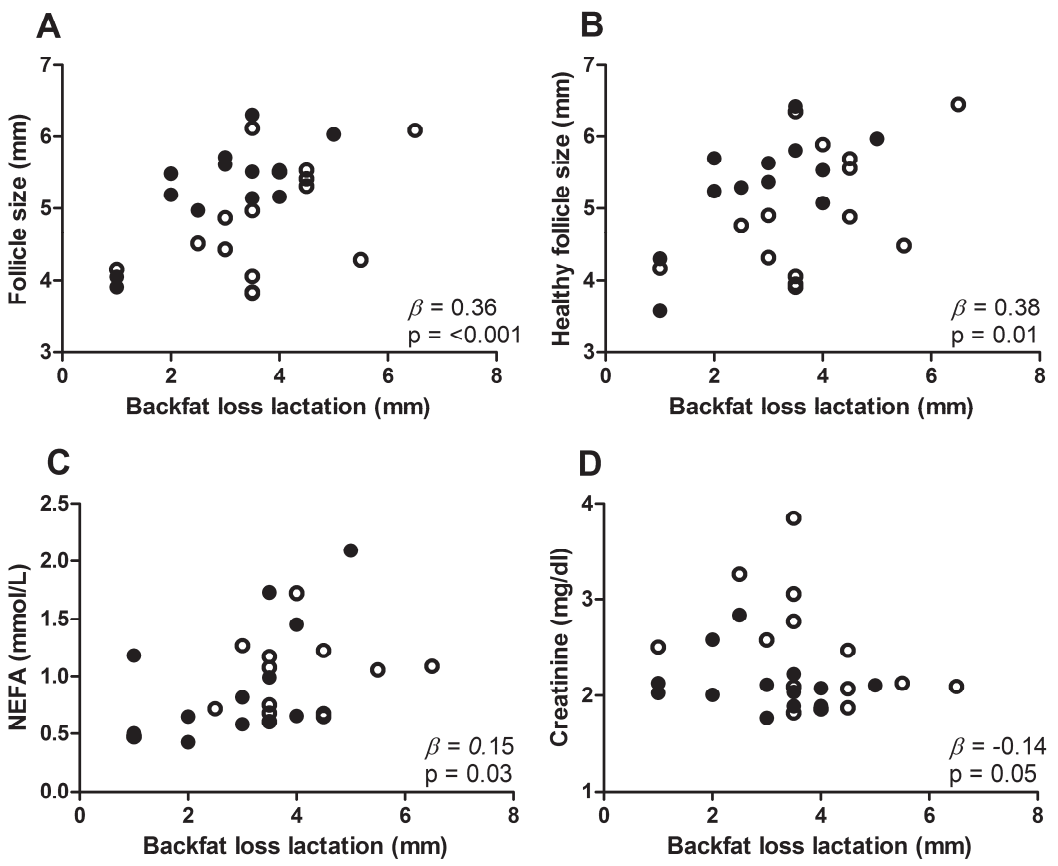

Figure 2.4 - Regression equations $(\beta)$ for the relation between backfat loss during lactation and (A) average follicle size of the 15 largest follicles, (B) average follicle size of the 10 largest healthy follicles, (C) serum non-esterified fatty acid (NEFA) levels ( $\mathrm{mmol} / \mathrm{L}$ ) at weaning and (D) serum creatinine $(\mathrm{mg} / \mathrm{dl})$ levels at weaning in sows. No interactions with parity class (PAR) have been found.

\section{Discussion}

We hypothesised that variation in the follicle pool may be a cause for variation in birth weight. In order to test this, sows were selected based on their EBV for variation in birth weight to apply a contrast in expected phenotypical variation in birth weight and to correlate this to variation in follicular development. We therefore have explored variation in follicular development in the follicle pool at weaning as from this antral follicle pool follicles will be recruited for ovulation to give rise to the next litter. This recruitment is due to the weaning associated change in pulsatile gonadotropin releasing hormone and $\mathrm{LH}$ patterns: these patterns change from a low frequency and high amplitude pattern to a high frequency and low amplitude pattern while FSH levels increase (Shaw and Foxcroft, 1985). Since it has been reported that the antral follicle pool is very heterogeneous regarding size and biochemical status in sows at 48 hours after weaning (Foxcroft et al., 1987), it is very well possible that variation in birth weight is caused by variation in the follicle pool at weaning.

The results of the present study do not support this assumption, as no relations could be found between (variation in) follicular development at weaning and EBV for variation in birth weight. One explanation for this unexpected finding may be that the contrast in EBV for variation in birth weight that we were able to obtain in this study or the heritability of the trait variation 
in birth weight (mean $\mathrm{h} 2=0.08$; Bidanel, 2011) might be too small to detect phenotypic differences with the present sample size ( $\mathrm{N}=14$ and $\mathrm{N}=15$ for High-EBV and Low-EBV, respectively). In addition, the repeatability of the trait variation in birth weight might be too low to relate the sows' previous performance in variation in birth weight to variation in follicular development of the follicle pool that will give rise to the next litter. Generally low repeatability's for variation in piglet birth weight are found in literature (0.14, Quesnel et al., 2008), although for the genetics used higher repeatability's (0.19) are seen (E.G. Knol, personal communication). Furthermore, we were able to obtain a difference between High-EBV and Low-EBV of 40.5 gram (15.8 \pm 1.6 vs. $-24.7 \pm 1.5)$ while the average EBV of all the sows on the nucleus farm was $-6.3 \pm 17$.1. In addition, no linear relations between EBV for variation in birth weight and follicular and metabolic parameters could be found which confirms that EBV for variation in birth weight was not related with any of the measured parameters.

Another explanation could be that variation in birth weight is not related to variation in the follicle pool at weaning but is related with variation in follicular development later in the follicular phase after recruitment. Following recruitment, follicles are either selected to ovulate or degenerate; indeed several studies have shown that many small and medium-sized antral follicles become atretic (reviewed by: Guthrie, 2005). It is therefore likely that some of the follicles that we have studied at weaning will become atretic in a later stage, will not ovulate and will therefore not be related to the EBV for variation in birth weights. Support for the assumption that follicular development after weaning may be an important phase in determining variation in birth weights comes from a study by van den Brand et al. (2006) which show that feeding insulin-stimulating diets in the post-weaning period can reduce variation in birth weights. On the other hand, Wientjes et al. (2013) show that more body weight and backfat loss during lactation is related to more variation in birth weight of the subsequent litter, which indicates that variation in birth weight could also originate from follicular development during lactation. These studies both indicate that sow metabolic status during and immediately after lactation can affect follicular development. Indeed, we observed phenotypic relations between the metabolic state and follicular development at weaning.

More body weight loss during lactation was related to lower plasma IGF1 levels and higher creatinine levels at weaning. This is expected since insulin and IGF1 are usually suppressed in catabolic states (Quesnel et al., 1998b; van den Brand et al., 2001), while creatinine levels are higher when sows lose more weight due to restricted feeding (Baidoo et al., 1992) or when they receive less lysine in their diet (Yang et al., 2009). Unexpectedly, we did not find any relations between weight loss during lactation and insulin levels at weaning. We also find that a larger body weight loss during lactation is related to a lower percentage healthy COCs. This corroborates findings by Zak et al. (1997), who reported decreased maturation rates of oocytes isolated 38 hours before the anticipated onset of oestrus in primiparous sows that were feed restricted from day 21-28 of lactation compared to sows that were fed ad libitum 
from day 21-28. We do not observe relations between body weight loss and follicle size. This is in contrast to a study by Quesnel et al. (1998a) who found that feed restriction during a 28day lactation period ( $50 \%$ ad lib vs. ad lib) resulted in smaller follicles at weaning in primiparous sows. Similar to our study, these investigators did not observe effects of feed restriction on follicular atresia.

Body weight loss consist of loss of fat mass or loss of lean mass. In general, sows lose around 5 -fold more kilograms of fat during lactation compared to protein (Bergsma et al., 2009). As sows lose both fat and protein simultaneously, it is difficult to establish which of the two is responsible for the negative effects of weight loss during lactation on follicular development. Some studies suggest that especially protein loss during lactation is detrimental for follicular development. In a study by Clowes et al. (2003a) first-parity sows were fed either 50, 35 or 24 g of lysine/day during a 23-day lactation period. The sows lost approximately 7, 9, and $16 \%$ of the calculated body protein mass while no differences in backfat loss were found. When the 8 largest follicles of both ovaries were analysed, it was found that sows which lost more protein during lactation had a lower percentage follicles larger than $4 \mathrm{~mm}$ at weaning $(23.6 \%$ vs. $55.4 \%$ for high vs. low protein loss) and follicles contained less follicular fluid ( $32 \mu \mathrm{l}$ vs. 68 $\mu \mathrm{l}$, respectively) with lower concentrations of estradiol and IGF1. In addition, the follicular fluid of protein restricted sows reduced oocyte maturation in vitro. Yang et al. (2000) found similar results to Clowes et al. (2003a) when analysing the 15 largest pre-ovulatory follicles at prooestrus in primiparous sows. Moreover, the severity of the effects of lysine restriction on follicle size and follicular fluid content is larger for sows with a lower calculated protein mass at parturition (Clowes et al., 2003b). In line with these studies, we find that increased creatinine levels, which can be considered a marker for protein loss (Yang et al., 2009), are related to a lower average follicle size of the 15 largest follicles at weaning. This indicates that increased protein loss during lactation has a negative effect on follicle size at weaning. We found no relation between urea levels, which is a marker for protein turnover, and follicle size.

In our study, the amount of fat loss during lactation was estimated by measuring backfat thickness after parturition and at weaning. Higher backfat loss during lactation is related to a higher backfat thickness at parturition. In addition, higher backfat loss is related to higher serum NEFA levels at weaning which, when measured in a fasted state as has been done in our study, is a marker for lipid mobilization (Lafontan and Langin, 2009). Together these findings suggest that the sows, which had more backfat at parturition, mobilised more lipid during lactation. Unexpectedly, in our study, no relations between the amount of backfat and leptin levels at weaning are found, in contrast to the findings of a study by De Rensis et al., (2005). In order to further elucidate relations between backfat loss during lactation, NEFA levels and follicular development at weaning, we measured FGF21, a hormone-like circulating protein recently identified as a metabolic regulator of glucose and lipid metabolism (reviewed by: Fisher and Maratos-Flier, 2016). We observe a tendency towards higher FGF21 levels in 
Low-EBV sows compared to High-EBV $(p=0.06)$. However, no relations between FGF21 and any of the other measured metabolic or follicular measurements can be found. More studies need to be performed to elucidate possible relations between FGF21 and follicular development.

As mentioned previously, it is not known to which extent negative effects of energy mobilization during lactation on follicular development, can be attributed to either protein or fat loss, since studies investigating effects of feed restriction on follicular development report a simultaneous loss of fat mass and lean mass. Most studies find that weight loss and backfat loss of sows during lactation, so the simultaneous loss of lean and fat mass, is related to a smaller follicle size (Quesnel et al., 1998a and Zak et al., 1997, respectively). Surprisingly, in our study, more backfat loss during lactation is related to a higher average size of the 15 largest follicles and higher average size of the 10 largest healthy follicles at weaning. In addition, higher serum NEFA levels at weaning tended to be related to a higher average follicle size at weaning. Together, these findings suggest that increased lipid mobilization during lactation is related to an increased follicle size.

One hypothesis for these surprising findings could be that sows which have low levels of backfat at parturition mobilise less backfat during lactation to fulfil the energy requirements of milk production and therefore have to use their protein reserves, which might have a detrimental effect on follicular development. Indeed, in our study, lower backfat loss during lactation was related to higher creatinine levels at weaning and high creatinine levels were related to a smaller follicle size at weaning. So, the relation between increased backfat loss during lactation and a larger average follicle size at weaning might be explained by protein sparing effects. It may be worthwhile to study relations between energy mobilization of different energy substrates during lactation and follicular development using reliable measurements of lean mass and fat mobilization, such as balance trials and body composition measurements.

To conclude, in this study, follicular development at weaning appeared to be similar for sows with a High vs. Low-EBV for variation in birth weight. It is possible that variation in birth weights is (partly) explained by variation in the follicle pool at weaning, but this is not reflected in EBV. Another possibility is that variation in birth weight is explained by follicle development at a later time point during the follicular phase or by other factors which play a role after ovulation. Our study does show that energy mobilization from different sources during lactation, adipose tissues or muscle reflecting fat or lean mass, respectively, could have divergent effects on follicular development at weaning. These relations need to be further elucidated. 


\section{Implications}

The metabolic state of lactating sows was monitored to assess phenotypic relations with follicular development at weaning. Lactational backfat loss was related to a higher backfat thickness at parturition and to a higher average follicle size at weaning. These results could implicate that sows with more backfat are able to mobilise more energy from backfat during lactation and could thereby spare protein reserves to support follicular development. A better understanding of the relation between energy mobilisation of different substrates during lactation in relation to follicular development could eventually be used for optimal breeding and feeding strategies for lactating sows.

\section{Acknowledgements}

We acknowledge the Wageningen Institute for Animal Sciences (WIAS) and NWO for providing the NWO-WIAS Graduate Programme 2015 grant. We are grateful to Quirijn Dees MSc for helping with the measurements during his MSc thesis.

\section{References}

Alvarez, G. M., Dalvit, G. C., Achi, M. V., Miguez, M. S., \& Cetica, P. D. (2009). Immature oocyte quality and maturational competence of porcine cumulus-oocyte complexes subpopulations. Biocell, 33(3), 167-177.

Baidoo, S., Aherne, F., Kirkwood, R., \& Foxcroft, G. (1992). Effect of feed intake during lactation and after weaning on sow reproductive performance. Canadian journal of animal science, 72(4), 911-917.

Bergsma, R., Kanis, E., Verstegen, M. W. A., van der Peet-Schwering, C. M. C., \& Knol, E. F. (2009). Lactation efficiency as a result of body composition dynamics and feed intake in sows. Livestock science, 125(2), 208-222.

Bidanel J. P. 2011. "Biology and Genetics of Reproduction". In The Genetics of the Pig $2^{\text {nd }}$ edition (pp. 218-241). CAB International, Wallingford, Oxfordshire, UK.

Clowes, E., Aherne, F., Foxcroft, G., \& Baracos, V. (2003a). Selective protein loss in lactating sows is associated with reduced litter growth and ovarian function. Journal of animal science, 81(3), 753-764.

Clowes, E., Aherne, F., Schaefer, A., Foxcroft, G., \& Baracos, V. (2003b). Parturition body size and body protein loss during lactation influence performance during lactation and ovarian function at weaning in first-parity sows. Journal of animal science, 81(6), 1517-1528.

da Silva, C. L. A., Laurenssen, B. F. A., Knol, E. F., Kemp, B., \& Soede, N. M. (2016). Relationships between ovulation rate and embryonic and placental characteristics in multiparous sows at 35 days of pregnancy. Animal, 10(7), 1192-1199.

De Rensis, F., Gherpelli, M., Superchi, P., \& Kirkwood, R. (2005). Relationships between backfat depth and plasma leptin during lactation and sow reproductive performance after weaning. Animal reproduction science, 90(1), 95-100.

Ferguson, E. M., Slevin, J., Edwards, S. A., Hunter, M. G., \& Ashworth, C. J. (2006). Effect of alterations in the quantity and composition of the pre-mating diet on embryo survival and foetal growth in the pig. Animal reproduction science, 96(1-2), 89-103.

Fisher, F. M., \& Maratos-Flier, E. (2016). Understanding the Physiology of FGF21. Annual review of physiology, 78, 223-241.

Fix, J., Cassady, J., Holl, J., Herring, W., Culbertson, M., \& See, M. (2010). Effect of piglet birth weight on survival and quality of commercial market swine. Livestock science, 132(1), 98-106. 
Foxcroft, G. R., Shaw, H. J., Hunter, M. G., Booth, P. J., \& Lancaster, R. T. (1987). Relationships between luteinizing hormone, follicle-stimulating hormone and prolactin secretion and ovarian follicular development in the weaned sow. Biology of reproduction, 36(1), 175-191.

Guthrie, H. (2005). The follicular phase in pigs: Follicle populations, circulating hormones, follicle factors and oocytes. Journal of animal science. Supplement, 83, 79-89.

Lafontan, M., \& Langin, D. (2009). Lipolysis and lipid mobilization in human adipose tissue. Progress in lipid research, 48(5), 275-297.

Milligan, B. N., Fraser, D., \& Kramer, D. L. (2002). Within-litter birth weight variation in the domestic pig and its relation to pre-weaning survival, weight gain, and variation in weaning weights. Livestock production science, 76(1), 181-191.

Patterson, J. L., Smit, M. N., Novak, S., Wellen, A. P., \& Foxcroft, G. R. (2011). Restricted feed intake in lactating primiparous sows. I. Effects on sow metabolic state and subsequent reproductive performance. Reproduction, fertility and development, 23(7), 889-898.

Pope, W., Wilde, M., \& Xie, S. (1988). Effect of electrocautery of nonovulated day 1 follicles on subsequent morphological variation among day 11 porcine embryos. Biology of reproduction, 39(4), 882-887.

Pope, W., Xie, S., Broermann, D., \& Nephew, K. (1989). Causes and consequences of early embryonic diversity in pigs. Journal of reproduction and fertility. Supplement, 40, 251-260.

Prunier, A., \& Quesnel, H. (2000). Influence of the nutritional status on ovarian development in female pigs. Animal reproduction science, 2(60-61), 185-197.

Quesnel, H., Pasquier, A., Mounier, A., \& Prunier, A. (1998a). Influence of feed restriction during lactation on gonadotropic hormones and ovarian development in primiparous sows. Journal of animal science, 76(3), 856-863.

Quesnel, H., Pasquier, A., Mounier, A.-M., Louveau, I., \& Prunier, A. (1998b). Influence of feed restriction in primiparous lactating sows on body condition and metabolic parameters. Reproduction nutrition development, 38(3), 261-274.

Shaw, H., \& Foxcroft, G. (1985). Relationships between LH, FSH and prolactin secretion and reproductive activity in the weaned sow. Journal of reproduction and fertility, 75(1), 17-28.

Slot, K. A., Voorendt, M., de Boer-Brouwer, M., van Vugt, H. H., \& Teerds, K. J. (2006). Oestrus cycle dependent changes in expression and distribution of Fas, Fas ligand, Bcl-2, Bax, and pro-and active caspase-3 in the rat ovary. Journal of endocrinology, 188(2), 179-192.

Tuchscherer, M., Puppe, B., Tuchscherer, A., \& Tiemann, U. (2000). Early identification of neonates at risk: traits of newborn piglets with respect to survival. Theriogenology, 54(3), 371-388.

Van den Brand, H., Dieleman, S. J., Soede, N. M., \& Kemp, B. (2000). Dietary energy source at two feeding levels during lactation of primiparous sows: I. Effects on glucose, insulin, and luteinizing hormone and on follicle development, weaning-to-estrus interval, and ovulation rate. Journal of animal science, 78(2), 396-404.

Van den Brand, H., Prunier, A., Soede, N. M., \& Kemp, B. (2001). In primiparous sows, plasma insulinlike growth factor-I can be affected by lactational feed intake and dietary energy source and is associated with luteinizing hormone. Reproduction nutrition development, 41(1), 27-39.

Van den Brand, H., Soede, N., \& Kemp, B. (2006). Supplementation of dextrose to the diet during the weaning to estrus interval affects subsequent variation in within-litter piglet birth weight. Animal reproduction science, 91(3), 353-358.

Van der Lende, T., Hazeleger, W., \& De Jager, D. (1990). Weight distribution within litters at the early foetal stage and at birth in relation to embryonic mortality in the pig. Livestock production science, 26(1), 53-65.

Wientjes, J. G. M., Soede, N. S., Knol, E. F., Van den Brand, H., \& Kemp, B. (2013). Piglet birth weight and litter uniformity: Effects of weaning-to-pregnancy interval and body condition changes in sows of different parities and crossbred lines. Journal of animal science, 91(5), 2099-2107.

Wientjes, J., Soede, N. M., Van der Peet-Schwering, C. M. C., Van Den Brand, H., \& Kemp, B. (2012). Piglet uniformity and mortality in large organic litters: Effects of parity and pre-mating diet composition. Livestock science, 144(3), 218-229. 
Xie, S., Broermann, D., Nephew, K., Bishop, M., \& Pope, W. (1990). Relationship between oocyte maturation and fertilization on zygotic diversity in swine. Journal of animal science, 68(7), 2027-2033.

Yang, H., Foxcroft, G., Pettigrew, J., Johnston, L., Shurson, G., Costa, A., \& Zak, L. (2000). Impact of dietary lysine intake during lactation on follicular development and oocyte maturation after weaning in primiparous sows. Journal of animal science, 78(4), 993-1000.

Yang, Y., Heo, S., Jin, Z., Yun, J., Choi, J., Yoon, S., Park, M., Yang, B., Chae, B. (2009). Effects of lysine intake during late gestation and lactation on blood metabolites, hormones, milk composition and reproductive performance in primiparous and multiparous sows. Animal reproduction science, 112(3), 199-214.

Zak, L. J., Xu, X., Hardin, R. T., \& Foxcroft, G. R. (1997). Impact of different patterns of feed intake during lactation in the primiparous sow on follicular development and oocyte maturation. Journal of reproduction and fertility, 110(1), 99-106.

\section{Supplemental Material}

Supplemental Table S2.1 - Effects of estimated breeding value classes for within-litter variation in piglet birth weight (estimated breeding value (EBV); High vs. Low) and parity classes (PAR; 3 vs. $4+5$ ) on follicular parameters of the 15 largest follicles of the left and right ovary and average follicle size of the 10 largest healthy follicles in sows. All values are presented as LS means.

\begin{tabular}{|c|c|c|c|c|c|c|c|}
\hline \multirow[b]{2}{*}{ Parameter } & \multicolumn{2}{|c|}{ EBV } & \multicolumn{2}{|c|}{ PAR } & \multirow[t]{2}{*}{ RMSE } & \multicolumn{2}{|c|}{ P-values ${ }^{1}$} \\
\hline & High & Low & 3 & $4+5$ & & EBV & PAR \\
\hline \multicolumn{8}{|l|}{ Right ovary } \\
\hline Av. follicle size $(\mathrm{mm})$ & 5.11 & 5.00 & 5.22 & 4.89 & 0.74 & 0.70 & 0.24 \\
\hline Av. follicle size $1-5(\mathrm{~mm})^{2}$ & 6.21 & 6.04 & 6.34 & 5.92 & 0.78 & 0.59 & 0.18 \\
\hline Av. follicle size $6-10(\mathrm{~mm})^{2}$ & 4.93 & 4.84 & 5.06 & 4.72 & 0.79 & 0.77 & 0.29 \\
\hline Av. follicle size $11-15(\mathrm{~mm})^{2}$ & 4.17 & 4.10 & 4.28 & 4.00 & 0.78 & 0.81 & 0.37 \\
\hline Variation (SD) in follicle size $(\mathrm{mm})$ & 1.01 & 0.93 & 1.03 & 0.92 & 0.27 & 0.41 & 0.30 \\
\hline $\begin{array}{l}\text { Av. follicle size of } 10 \text { largest } \\
\text { healthy follicles }(\mathrm{mm})\end{array}$ & 5.18 & 5.06 & 5.27 & 4.97 & 0.83 & 0.73 & 0.39 \\
\hline Healthy follicles (\%) & 64.8 & 66.0 & 67.8 & 66.6 & 17.9 & 0.73 & 0.87 \\
\hline \multicolumn{8}{|l|}{ Left ovary } \\
\hline Healthy COCs (\%) & 71.6 & 73.2 & 76.8 & 67.9 & 21 & 0.85 & 0.32 \\
\hline Follicular fluid $(\mu \mathrm{l})$ & 375 & 367 & 421 & 321 & 149 & 0.89 & 0.10 \\
\hline
\end{tabular}

$\mathrm{COCs}=$ cumulus-oocyte complexes. ${ }^{1}$ Interactions between EBV and parity were never significant. ${ }^{2}$ Average follicle size of the largest follicles (follicles 1-5), medium-sized follicles (follicles 6-10) and smallest follicles (follicles 1115 ) of the 15 largest follicles. 
Supplemental Table S2.2 - Effects of average sow follicle size classes (FS; Small $<5.0 \mathrm{~mm}(\mathrm{~N}=15)$ vs. Large $>5.1 \mathrm{~mm}(\mathrm{~N}=14))$ and parity classes (PAR; $3(\mathrm{~N}=14)$ vs. $4+5(\mathrm{~N}=15))$ on follicular parameters of the 15 largest follicles of the left and right ovary. All values are presented as LS means.

\begin{tabular}{|c|c|c|c|c|c|c|c|}
\hline \multirow[b]{2}{*}{ Parameter } & \multicolumn{2}{|c|}{ FS } & \multicolumn{2}{|c|}{ PAR } & \multirow[t]{2}{*}{ RMSE } & \multicolumn{2}{|c|}{ P-values ${ }^{1}$} \\
\hline & Small & Large & 3 & $4+5$ & & FS & PAR \\
\hline \multicolumn{8}{|l|}{ Right ovary } \\
\hline Av. follicle size $(\mathrm{mm})$ & 4.43 & 5.62 & 5.07 & 4.98 & 0.41 & $<0.001$ & 0.59 \\
\hline Av. follicle size 1-5 $(\mathrm{mm})^{2}$ & 5.49 & 6.70 & 6.19 & 6.01 & 0.48 & $<0.001$ & 0.34 \\
\hline Av. follicle size $6-10(\mathrm{~mm})^{2}$ & 4.22 & 5.50 & 4.89 & 4.84 & 0.44 & $<0.001$ & 0.77 \\
\hline Av. follicle size $11-15(\mathrm{~mm})^{2}$ & 3.56 & 4.67 & 4.13 & 4.10 & 0.54 & $<0.001$ & 0.88 \\
\hline Variation $(\mathrm{SD})$ in follicle size $(\mathrm{mm})$ & 0.94 & 1.00 & 1.03 & 0.91 & 0.27 & 0.56 & 0.24 \\
\hline $\begin{array}{l}\text { Av. follicle size of } 10 \text { largest } \\
\text { healthy follicles }(\mathrm{mm})\end{array}$ & 4.36 & 5.71 & 5.02 & 5.05 & 0.48 & $<0.001$ & 0.87 \\
\hline Healthy follicles (\%) & 61.3 & 72.5 & 66.5 & 67.3 & 16.9 & 0.10 & 0.90 \\
\hline \multicolumn{8}{|l|}{ Left ovary } \\
\hline Healthy COCs (\%) & 75.7 & 69.6 & 77.7 & 67.6 & 21 & 0.47 & 0.24 \\
\hline Follicular fluid $(\mu l)$ & 334 & 405 & 412 & 327 & 145 & 0.21 & 0.14 \\
\hline
\end{tabular}

COCs = cumulus-oocyte complexes. ${ }^{1}$ Interactions between FS and PAR were never significant. ${ }^{2}$ Average follicle size of the largest follicles (follicles 1-5), medium-sized follicles (follicles 6-10) and smallest follicles (follicles 1115) of the 15 largest follicles.

Supplemental Table S2.3 - Effects of average sow variation (SD) in follicle size classes (VARFS; Small $<0.09 \mathrm{~mm}(\mathrm{~N}=14)$ vs. Large $>0.09 \mathrm{~mm}(\mathrm{~N}=15))$ and parity classes (PAR; $3(\mathrm{~N}=14)$ vs. $4+5(\mathrm{~N}=15))$ on follicular parameters of the 15 largest follicles of the left and right ovary. All values are presented as LSmeans.

\begin{tabular}{|c|c|c|c|c|c|c|c|}
\hline \multirow[b]{2}{*}{ Parameter } & \multicolumn{2}{|c|}{ VARFS } & \multicolumn{2}{|c|}{ PAR } & \multirow[t]{2}{*}{ RMSE } & \multicolumn{2}{|c|}{ P-values ${ }^{1}$} \\
\hline & Small & Large & 3 & $4+5$ & & VARFS & PAR \\
\hline \multicolumn{8}{|l|}{ Right ovary } \\
\hline Av. follicle size $(\mathrm{mm})$ & 5.02 & 5.07 & 5.23 & 4.87 & 0.74 & 0.86 & 0.21 \\
\hline Av. follicle size $1-5(\mathrm{~mm})^{2}$ & 5.84 & 6.38 & 6.28 & 5.94 & 0.73 & 0.07 & 0.23 \\
\hline Av. follicle size $6-10(\mathrm{~mm})^{2}$ & 4.87 & 4.91 & 5.07 & 4.71 & 0.79 & 0.91 & 0.25 \\
\hline Av. follicle size $11-15(\mathrm{~mm})^{2}$ & 4.36 & 3.93 & 4.34 & 3.95 & 0.75 & 0.15 & 0.17 \\
\hline Variation (SD) in follicle size $(\mathrm{mm})$ & 0.74 & 1.18 & 0.98 & 0.95 & 0.15 & $<0.001$ & 0.60 \\
\hline $\begin{array}{l}\text { Av. follicle size of } 10 \text { largest } \\
\text { healthy follicles }(\mathrm{mm})\end{array}$ & 5.18 & 5.06 & 5.29 & 4.95 & 0.8 & 0.70 & 0.30 \\
\hline Healthy follicles (\%) & 72.5 & 62.2 & 69.6 & 65.2 & 17.1 & 0.13 & 0.51 \\
\hline \multicolumn{8}{|l|}{ Left ovary } \\
\hline Healthy COCs (\%) & 67.1 & 76.7 & 74.7 & 69.2 & 21 & 0.26 & 0.51 \\
\hline Follicular fluid $(\mu l)$ & 336 & 404 & 412 & 327 & 145 & 0.23 & 0.14 \\
\hline
\end{tabular}

COCs = cumulus-oocyte complexes. ${ }^{1}$ Interactions between VARFS and PAR were never significant. ${ }^{2}$ Average follicle size of the largest follicles (follicles 1-5), medium-sized follicles (follicles 6-10) and smallest follicles (follicles 11-15) of the 15 largest follicles. 

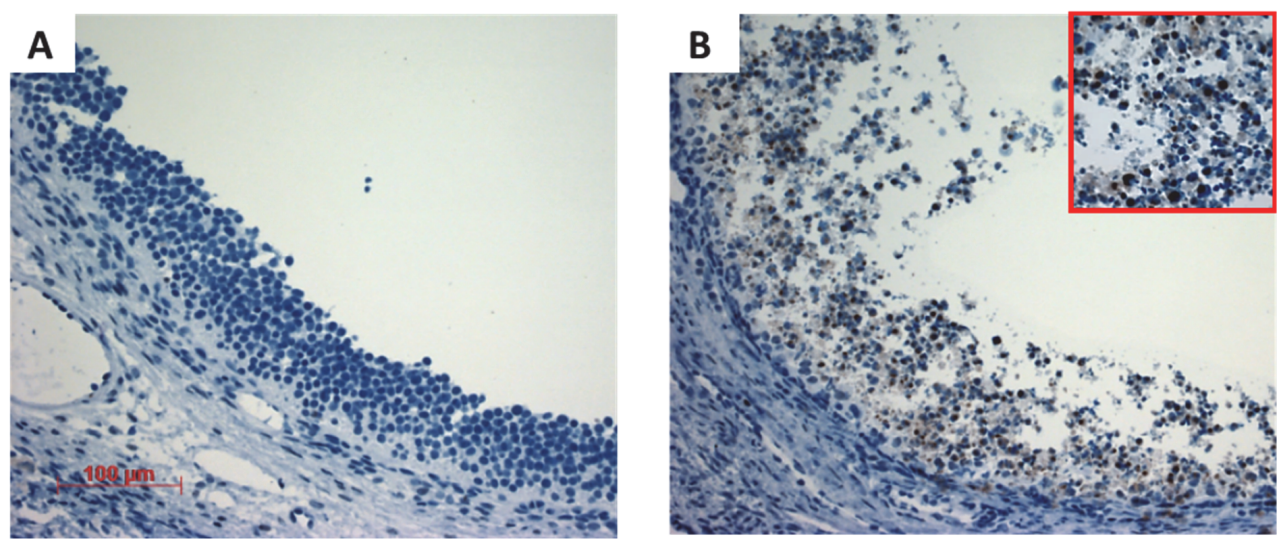

Supplemental Figure \$2.1 - Immunostaining for cleaved-Caspase 3 as a marker of cells in apoptosis and used to determine percentage healthy follicles in sows. Representative staining of (A) healthy and (B) unhealthy follicles under 20x magnification (insert $40 \times$ magnification) using light microscopy (Axioskop 2, Carl Zeiss Microscopy, Thornwood, NY, US) and imaged using imaging software (Axiovision 4.8, Carl Zeiss Microscopy).

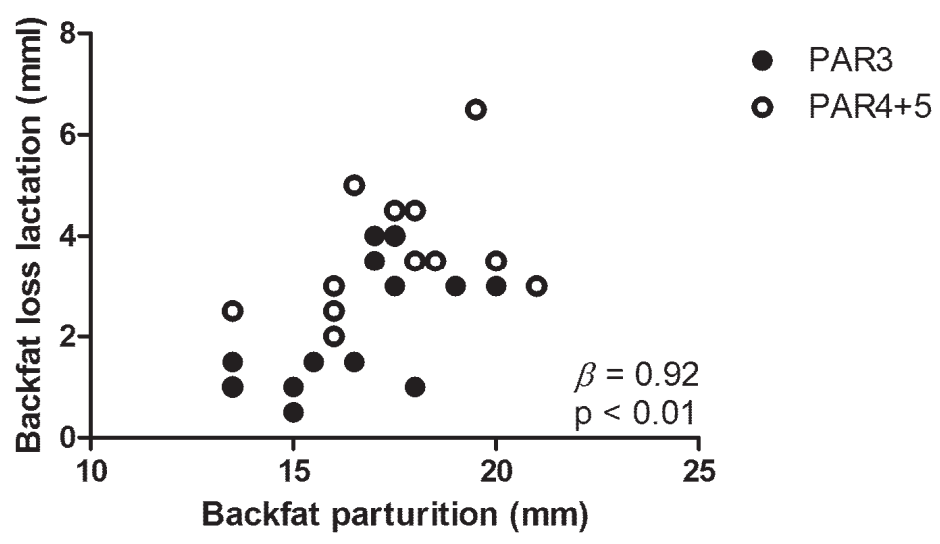

Supplemental Figure S2.2 - Regression equation $(\beta)$ for the relation between backfat thickness at parturition $(\mathrm{mm})$ and backfat loss during lactation $(\mathrm{mm})$. No interactions with parity class (PAR) have been found. 


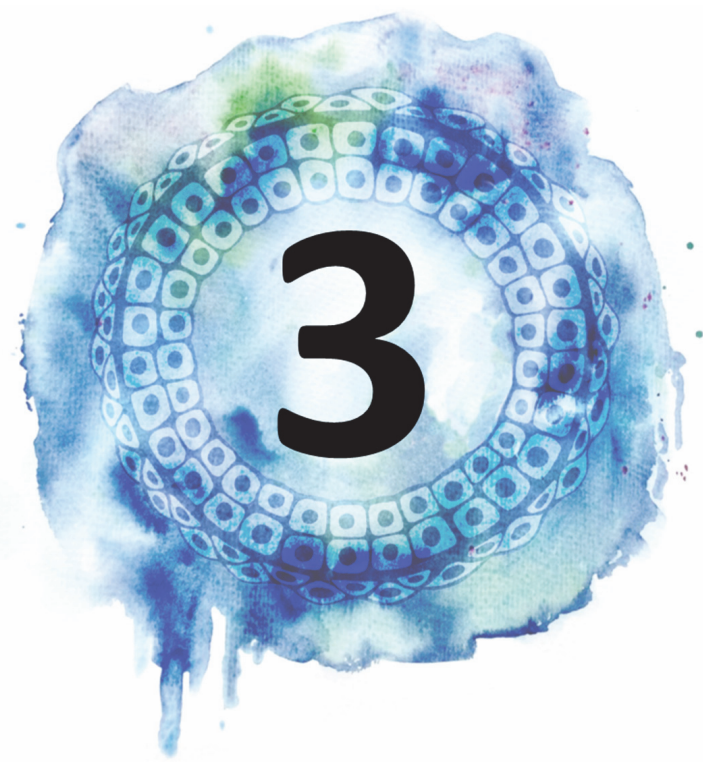

\title{
In ovaries with high or low variation in follicle size, granulosa cells of antral follicles exhibit distinct size-related processes
}

\author{
Natasja G. J. Costermans ${ }^{1,2}$, Jaap Keijer ${ }^{1}$, Evert M. van Schothorst ${ }^{1}$, \\ Bas Kemp ${ }^{2}$, Shohreh Keshtkar ${ }^{3}$, Annelies Bunschoten ${ }^{1}$, \\ Nicoline M. Soede ${ }^{2} \&$ Katja J. Teerds ${ }^{1}$
}

${ }^{1}$ Human and Animal Physiology, Wageningen University and Research, The Netherlands

${ }^{2}$ Adaptation Physiology Group, Wageningen University and Research, The Netherlands ${ }^{3}$ Nutrition, Metabolism \& Genomics Group, Wagenigen University and Research, The Netherlands 



\begin{abstract}
Antral follicle size might be a valuable additive predictive marker for IVF outcome. To better understand consequences of antral follicle size as a marker for reproductive outcome, we aimed to obtain insight in follicle size-related granulosa cell processes, as granulosa cells play an essential role in follicular development via the production of growth factors, steroids and metabolic intermediates. Using the pig as a model, we compared gene expression in granulosa cells of smaller and larger follicles in the healthy antral follicle pool of sows which had a high variation versus low variation in follicle size. Selected gene expression was confirmed at the protein level. Granulosa cells of smaller antral follicles showed increased cell proliferation, which was accompanied by a metabolic shift towards aerobic glycolysis (i.e. the Warburg effect), similar to other highly proliferating cells. High granulosa cell proliferation rates in smaller follicles might be regulated via increased granulosa cell expression of the androgen receptor and the epidermal growth factor receptor, which are activated in response to locally produced mitogens. While granulosa cells of smaller follicles in the pool are more proliferative, granulosa cells of larger follicles express more maturation markers such as insulin-like growth factor 1 (IGF1) and angiopoietin 1 (ANGPT1) and are therefore more differentiated. As both higher IGF1 and ANGPT1 have been associated with better IVF outcomes, the results of our study imply that including smaller follicles for oocyte aspiration might have negative consequences for IVF outcome.
\end{abstract}

Keywords: granulosa cells, follicle development, follicle size, porcine, gene expression, IVF, metabolism 


\section{Introduction}

Ovarian follicular recruitment can be subdivided in two phases that are differentially controlled. During initial ovarian follicular recruitment, primordial follicles are continuously selected to enter the growing pool of follicles. These follicles grow to the early antral stage to form a pool of small antral follicles. Subsequently, small antral follicles are selected from this pool to develop into pre-ovulatory follicles. The follicles selected during the follicular phase of the oestrus/menstrual cycle will finally ovulate to produce viable offspring (reviewed by Oktem and Urman, 2010). The size of the small antral follicle pool and the size of individual antral follicles in the pool vary between individuals, which may be explained by factors such as age, nutrition and genetic background (Moslehi et al., 2017; Vanden Brink et al., 2015).

Antral follicle count during the follicular phase using ultrasonography is currently used in clinical practice to predict IVF outcome and appropriate timing for hCG administration (Christianson et al., 2015). Some studies show that a larger antral follicle size might be a valuable additive predictive marker for ovarian response to gonadotropin administration and subsequent IVF outcome (Lai et al., 2013; Schumacher et al., 2018), while other studies do not find relations between follicle size and IVF outcome (Knopman et al., 2012). It is therefore important to better understand the consequences of follicle size for reproductive outcome. Granulosa cells play an essential role in follicular development, as they are responsible for the production of growth factors and steroids needed for follicular growth and survival (reviewed by Matsuda et al., 2012). Granulosa cells also provide the oocyte with the necessary growth factors, nutrients and metabolic intermediates allowing the oocytes to obtain their full developmental competence (Jiang et al., 2010; Sugiura et al., 2005).

Pigs have a very well-defined start of the follicular phase, as follicular growth is suppressed during lactation by the suckling stimulus of piglets, and is initiated at weaning via the removal of this inhibitory stimulus (Soede et al., 2011). Therefore, poly-ovulatory pigs at the start of the follicular phase, which is $5-6$ days before ovulation in sows, were used as an animal model. This enables the study of differences in follicle size within the antral follicle pool, which potentially will give rise to the next batch of ovulatory follicles.

The aim of this study was to better understand relations between antral ovarian follicle size and reproductive outcome, by determining granulosa cell processes that correlate with antral follicle size. This was done by studying differential gene and protein expression in granulosa cells of smaller and larger healthy antral follicles at the start of the follicular phase of the oestrus cycle immediately after weaning of the piglets. This enables analysis of granulosa cell processes in different sized follicles in the pool in ovaries, which were in the same phase of the oestrus cycle. As follicles consist of different cell types, e.g. theca cells, mural granulosa cells, cumulus cells and the oocyte, we used laser-capture microdissection (LCM) to obtain pure samples of mural granulosa cells for whole-genome transcriptome analysis. In sows, the early antral follicle pool is very heterogeneous in e.g. size and steroid content of 
the follicular fluid (Foxcroft et al., 1987; Hunter and Wiesak, 1990). This follicular heterogeneity is also found in human pre-ovulatory follicles (Carpintero et al., 2015). To which extent this variety contributes to the developmental competence of the follicles is not clear. Therefore, sows with high variation in antral follicle size as well as sows with low variation in antral follicle size were used for comparisons.

\section{Methods}

\section{Animals}

The experiment was approved by the Animal Care and Use Committee of Wageningen University (DEC2016036) and performed according to national and EU law and guidelines. For this study, a total of 29 multiparous Dutch Landrace sows (parity 3 to 5; Topigs Norsvin, Vught, the Netherlands) were used. The sows were fed a standard lactation diet (ca. $12.5 \mathrm{MJ}$ $\mathrm{NE} / \mathrm{kg}, 154 \mathrm{~g} / \mathrm{kg} \mathrm{CP}, 9.3 \mathrm{~g} / \mathrm{kg}$ total lysine; Lacto Excellent, Agrifirm, Apeldoorn, The Netherlands). Within 24 hours after parturition, piglets were cross fostered to ensure 13 suckling piglets per sow. Sows had a lactation period of $26.1 \pm 0.2$ ( 25 to 27 ) days and weaned $12.7 \pm 0.4$ piglets. The sows were slaughtered at the slaughterhouse by stunning and exsanguination within 2 hours after weaning.

\section{Measurements}

\section{Follicular measurements}

The experimental procedure used for follicular measurements is summarized in Fig. 3.1. After slaughter, the right ovary of all 29 sows was cut in 2 halves, immediately frozen in liquid nitrogen and stored at $-80^{\circ} \mathrm{C}$ until further analysis. These 2 halves were used to measure follicle size and to assess if the follicles were healthy or atretic. Of each half of the ovary, 3 cutting planes were determined in order to make sections containing (almost all) antral follicles on the surface of the ovary. The ovaries were held against a ruler and a photograph was taken from three different sides. These photographs were used for later follicle size determination. Follicle size was determined as the largest macroscopically visible diameter of the follicle using ImageJ (version 1.51f, National Institutes of Health, USA). To determine if these follicles were healthy or atretic, cryo-sections of the right ovary were made and stained immunohistochemically for the presence of cleaved-Caspase 3 (a marker for cells in apoptosis) as described (Costermans et al., 2019). Follicle size and health status were used to determine average follicle size and the variation in follicle size of the 10 largest healthy follicles per ovary. These 10 follicles are assumed to represent the ovulatory follicle pool, as ovulation rates (of the left and right ovary) in modern sows are around 25-30 (Da Silva et al., 2017). To study within-ovary follicle size effects, 8 sows were selected that had a high or low variation (SD) in follicle size (HighVAR: $S D=1.56 \pm 0.18 \mathrm{~mm}$ versus LowVAR: $\mathrm{SD}=$ $0.54 \pm 0.16 \mathrm{~mm})$ and a similar average follicle size $(5.2 \pm 0.7 \mathrm{~mm}$ versus $5.5 \pm 0.9 \mathrm{~mm}$ for HighVAR and LowVAR, respectively; Fig. 3.2A). 


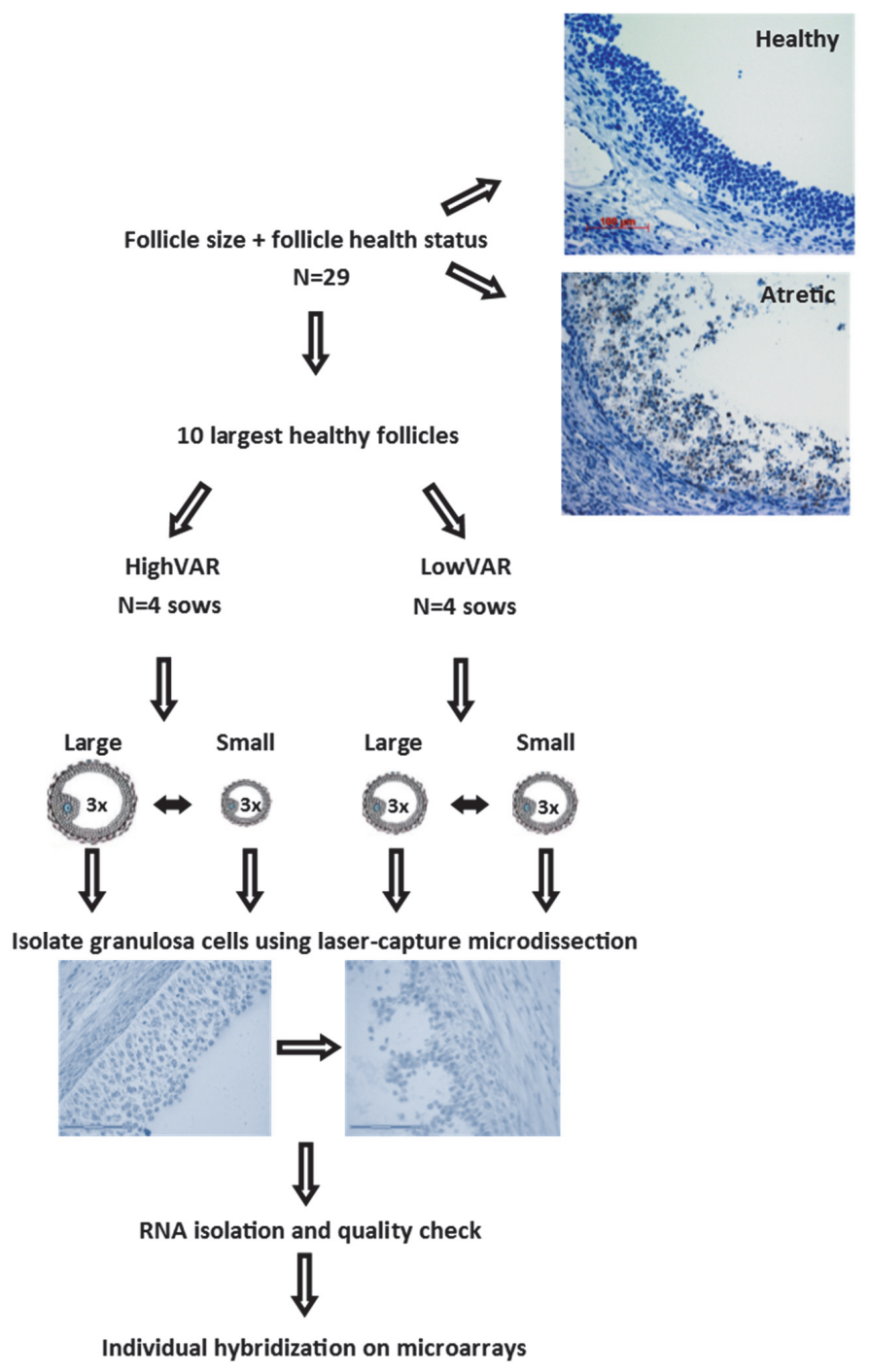

Figure 3.1 - Overview of experimental design

\section{Isolation of granulosa cells}

Granulosa cells of the 3 biggest and 3 smallest healthy follicles from the 10 largest healthy follicles of each of the 8 animals were isolated using laser-capture microdissection similar to Field et al. (2011). In short, $10 \mu \mathrm{m}$ cryo-sections of the selected follicles were mounted on Superfrost glass slides (Menzel- Gläser, Braunschweig, Germany) and air-dried for 10 minutes. The sections were fixed for $2 \times 2$ minutes in $100 \%$ ethanol, dipped in water and counterstained with Mayer's haematoxylin (Klinipath, Duiven, The Netherlands). The sections were dehydrated $2 \times 2$ minutes in $100 \%$ ethanol and subsequently incubated for $2 \times 2$ minutes in xylene. For each sample, around $1 \times 10^{4}$ granulosa cells were captured under $20 x$ magnification 
using Arcturus Pixcell II laser capture microdissection system (ThermoFisher scientific, Waltham, MA, USA) onto transfer film coated LCM caps (Thermo CapSure HS LCM Caps, ThermoFisher scientific). Within 10 minutes after microdissection, the transfer films were removed from the caps and lysed with $300 \mu \mathrm{l}$ of extraction buffer (Picopure RNA Isolation kit, Arcturus, ThermoFisher scientific) and incubated for $30 \mathrm{~min}$ at $42^{\circ} \mathrm{C}$. The resulting cell lysates were stored at $-80^{\circ} \mathrm{C}$ until RNA isolation was performed.

\section{RNA isolation}

Total RNA from the granulosa cell lysates was extracted using the Picopure RNA Isolation kit (Arcturus, ThermoFisher scientific), including an on-column DNAse treatment (Qiagen, Venlo, The Netherlands). RNA concentration and purity were measured with a NanoDrop spectrophotometer (IsoGen Life Sciences, Maarsen, The Netherlands) and integrity of a subset of randomly selected RNA samples was checked using the Agilent 2200 Tapestation (Agilent Technologies Inc., Santa Clara, CA, USA). Approximately 40 ng of RNA was used as input material for subsequent transcriptome analysis.

\section{Whole-genome transcriptome analysis}

Granulosa cell samples were individually hybridized on $4 \times 44 \mathrm{~K}$ whole-genome gene expression arrays (porcine; G2519F-026440, Agilent). The procedure was conducted as described by the manufacturer's protocol with a few modifications, as published (van Schothorst et al., 2007). Quantification of signals was performed using Feature Extraction version 10.7.3.1 (Agilent) as published (Hoek-van den Hil et al., 2013), followed by a quality control. Based on this quality control, one $4 \times 44$ array slide was excluded from further analysis. Normalization was performed as published (Hoek-van den Hil et al., 2013). Microarray data have been deposited in NCBI Gene Expression Omnibus (GEO) under accession number GSE125189. Fold-change (FC) is expressed as the ratio of the normalised expression of small follicles over large follicles. Student's $t$ test was used with false discovery rate (FDR) adjustment for multiple testing correction according to Benjamini-Hochberg. Individual transcripts with a FDR-adjusted $p \leq$ 0.1 and $F C \geq 1.2$ were considered significant. To identify which processes are regulated we used a FDR $\leq 0.2$ cut-off as only a sufficient number of genes in a pathway allows identification of a pathway. This cut-off was estimated using the methodology of Benjamini and Hochberg: FDR-adjusted $\mathrm{p}$-value $=\mathrm{P}(\mathrm{m}) * \mathrm{~N} / \mathrm{m}$, where $\mathrm{P}=\mathrm{p}$-value of $0.05, \mathrm{~N}=$ total number of analysed genes and $m=$ number of significantly expressed genes. In our dataset, $0.05 * 11409 / 3057$ yielded an estimated FDR of 0.194 . Thus, for the pathway analysis only, we included genes with a FDR $\leq 0.2$ and FC $\geq 1.2$. We only used this value for pathway analysis, since for the analysis of individual genes a p-value of 0.05 (without concomitant FC cut-off) could mean that $20 \%$ of the genes could be false positives. To validate this approach, we have additionally performed pathway analysis including only genes with an $F D R \leq 0.1$. Analysis of networks of genes was performed using Reactome (http://www.reactome.org), where non-assigned probes were excluded from further analysis. 


\section{$R T$-qPCR validation}

Granulosa cell samples of large and small follicles of HighVAR sows were used for RT-qPCR validation of two target genes, $A M H$ and $P G K 1$. cDNA synthesis (iScript CDNA synthesis kit, Bio$\mathrm{rad}$, Veenendaal, The Netherlands kit) was done followed by 12 cycles of pre-amplification (sSO advanced, PreAmp Supermix, Bio-rad) and RT-qPCR was performed as described (Almeida et al., 2018). Expression was normalized by stable expression of LAMP1 and ACTB. Primer sequences used for AMH: (forward) '5-AAGCTCCTCATCAGCCTGTCT-3' and (reverse) '5ATtGGGGCGATCGGGTTTG-3', PGK1: '3-AATCTGCCAAAGAAGGCTGGA-3' and 5'TAAAGGCCATTCCACCACCAA-3', LAMP1: '5- TCCAGATACAACGTGAGCGG-3' and '5CTCGTGTTCAGGGCAAACTG-3' and ACTB: '3-ACTGGCATTGTCATGGACTCTG-3' and 5'AGTTGAAGGTGGTCTCGTGGAT-3'. Data was analysed with a Student's t-test (for PGK1 after log transformation to obtain normality) in GraphPad Prism (version 5.04, GraphPad Software, La Jolla, CA, USA).

\section{Immunofluorescent staining}

Immunofluorescent staining was performed to quantify protein expression of Ki-67 and PKM2. For this analysis, the largest and smallest follicle of the 10 largest healthy follicles were analysed for each of the 8 selected sows. Cryosections were mounted on Superfrost plus glass slides (Menzel), air-dried for 30 minutes and fixed in $4 \%(v \backslash v)$ paraformaldehyde for 10 minutes. Heat-mediated epitope antigen retrieval was performed by washing in $\mathrm{H}_{2} \mathrm{O}$, microwaving the slides for 10 min under sub-boiling conditions in $0.1 \mathrm{M}$ sodium citrate buffer $(\mathrm{pH} 6)$, followed by cooling down to room temperature and rinsing in $\mathrm{PBS} \mathrm{pH}$ 7.4. Aldehyde residues were blocked with $0.3 \%$ glycine in PBS for 20 min. After rinsing with PBS, sections were pre-incubated with $5 \%$ (v/v) normal goat serum (Vector Laboratories, Peterborough, UK) in PBS for 60 min at room temperature. Subsequently, the sections were incubated overnight at $4{ }^{\circ} \mathrm{C}$ in a humid chamber with primary antibodies diluted in PBS-BSA-C (Aurion, Wageningen, The Netherlands); rabbit polyclonal anti-Ki-67 (1:500, ab15580, Abcam, Cambridge, UK), or rabbit monoclonal anti-PKM2 (1:100, 4053S, Cell Signalling Technology, Danvers, MA, USA). Next, sections were rinsed with PBS and treated with a fluorochrome labelled goat-anti-rabbit antibody (Alexa fluor 488, Invitrogen, CA, USA) diluted 1:200 (v/v) in PBS-BSA-c for 1 hour at room temperature. Sections were counterstained with DAPI $(1 \mu \mathrm{g} / \mathrm{ml}$; Sigma-Aldrich, Saint Louis, MO, USA) for 10 minutes and imaged at 10 times magnification using fluorescence microscopy (Leica DM6B), a digital camera (DFC365 FX) and imaging software (LasX; all Leica Microsystems, Amsterdam, The Netherlands). The mean staining intensity (sum of gray values in all pixels divided by the number of pixels selected) was determined for all parts of the granulosa cell layer that were completely intact, as determined visually, by ImageJ. A repeated measures ANOVA was used to compare the quantity of staining in smaller versus larger follicles in HighVAR and LowVAR sows using GraphPad Prism. 


\section{Results}

Global gene expression in granulosa cells was compared between the smaller and larger healthy antral follicles within the same follicle pool. For both HighVAR and LowVAR sows, the 3 smallest and 3 largest healthy follicles of the 10 largest healthy follicles were selected for transcriptome analysis. Average follicle size of the 3 smallest and 3 largest healthy follicles was $3.1 \pm 0.9 \mathrm{~mm}$ versus $6.9 \pm 0.9 \mathrm{~mm}$ for the HighVAR sows and was $5.0 \pm 1.1 \mathrm{~mm}$ versus $6.2 \pm 0.8 \mathrm{~mm}$ for the LowVAR sows (Fig. 3.2A and B).
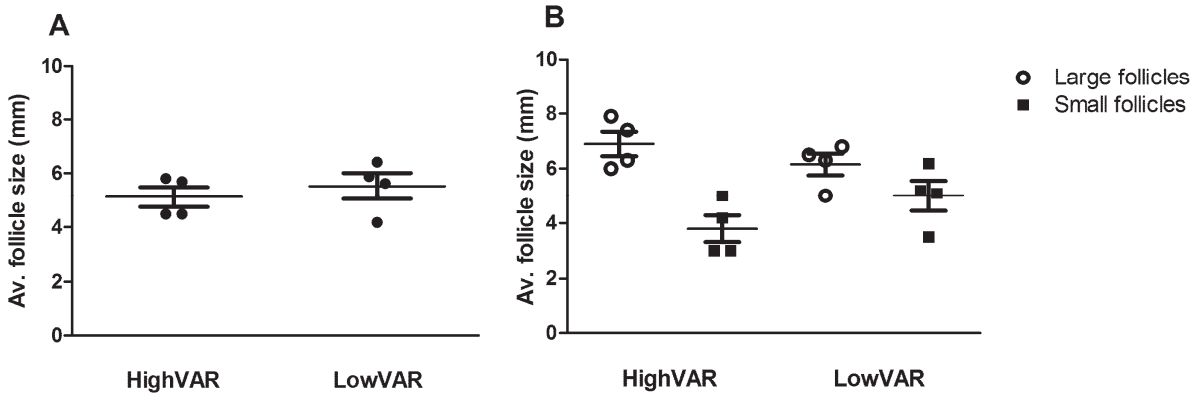

Figure 3.2 - (A) Average follicle size of the 10 largest healthy follicles of sows with high variation in follicle size (HighVAR) versus low variation (LowVAR) (B) Average follicle size of the 3 smallest and 3 largest follicles of the 10 largest healthy follicles of HighVAR versus LowVAR sows which were selected for transcriptome analysis. Data are presented as average follicle size of each sow (dots) and average follicle size of the 4 sows \pm SD (bar).

HighVAR sows had more differentially expressed genes (645) between the granulosa cells of the smaller and larger follicles compared to LowVAR sows (97). Of these genes, a core-subset of 75 genes was differentially expressed in both HighVAR and LowVAR (Fig. 3.3A). Gene expression of two mRNAs, phosphoglycerate kinase 1 (PGK1, a glycolytic enzyme) and antiMüllerian hormone (AMH, a follicular growth factor) was confirmed by RT-qPCR (Supplemental Fig. S3.1; AMH: FC $=1.67$ versus 2.07 and $p<0.01$ versus $p=0.02$ and PGK1: $F C=1.82$ versus 1.83 and $p<0.01$ versus $p=0.07$ for micro-array and RT-qPCR, respectively). For pathway analysis only, we have included all genes with a FDR $\leq 0.2$ and $F C \geq 1.2$. The coresubset of genes differently expressed in both HighVAR and LowVAR consisted of 349 genes and was used to identify pathways differing between the smaller and larger follicles. This pathway analysis revealed that 9 of the 10 top pathways were involved in cell cycle progression (Fig. 3.3B). In addition, we performed a more stringent pathway analysis by only including genes with a FDR $<0.10$ and identified similar processes, with 7 of the 10 top pathways involved in cell cycle progression (see Supplemental Table S3.1). This agrees with subsequent analysis of individual transcripts, which also showed a higher expression of genes related to cell cycle progression in granulosa cells of smaller versus larger follicles (top 10 of the most regulated genes in smaller and larger follicles can be found in Supplemental Table S3.2). Of special interest is histone H2A.Z (H2AFZ), which can accelerate cell cycle transition 
and is required for estrogen dependent proliferation (Svotelis et al., 2010). Another gene of interest is cyclin dependent kinase 1 (CDK1), which is the essential cell cycle CDK for cell proliferation (Santamaría et al., 2007). Immunostaining for the proliferation marker Ki-67 showed a higher expression in smaller versus larger follicles for both LowVAR and HighVAR sows (Fig. 3.4A).

A Number of significantly different genes

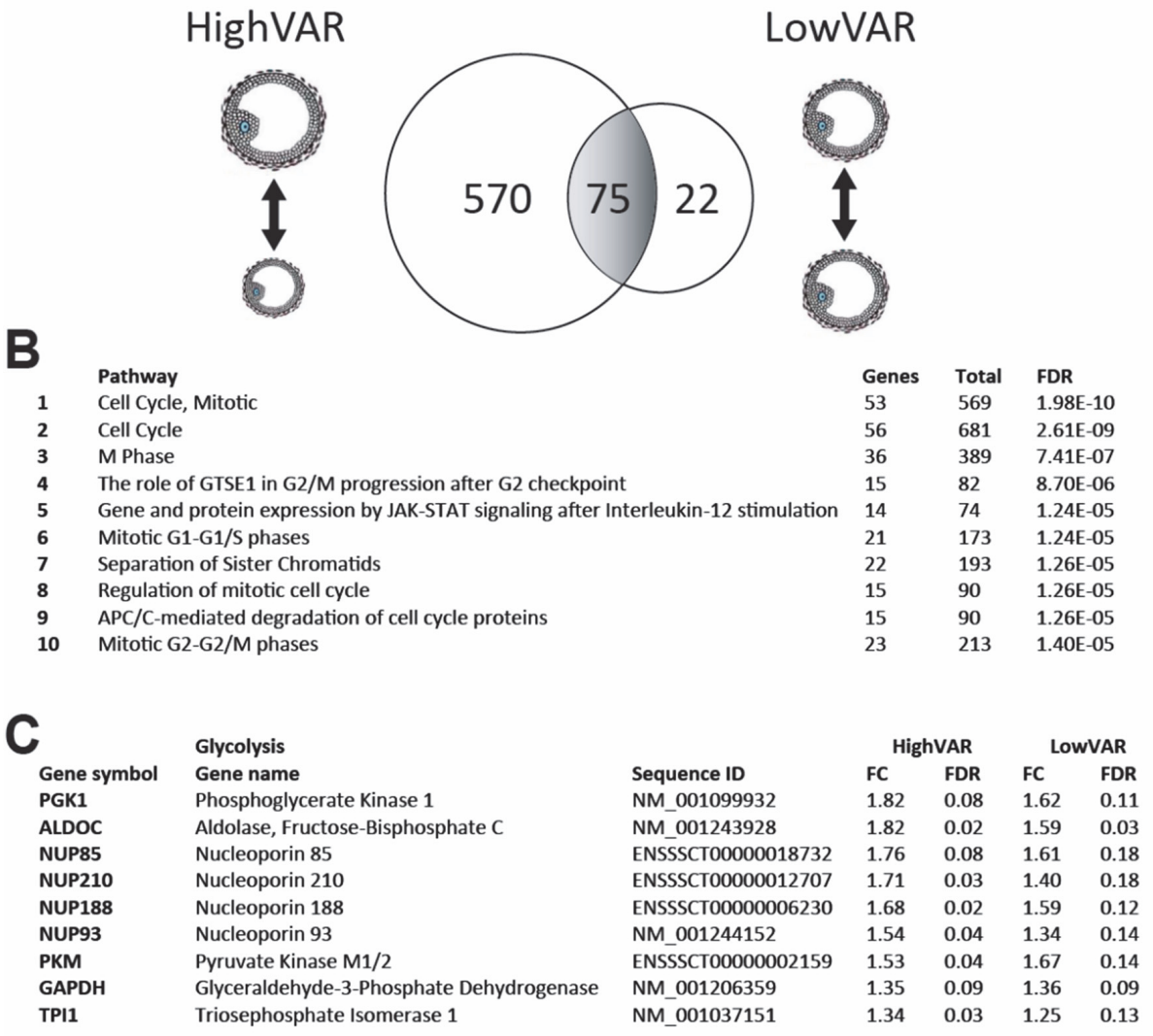

Figure 3.3 - Gene expression in smaller versus larger follicles in sows with high variation in follicle size (HighVAR) versus low variation (LowVAR). (A) Venn diagram showing the number of genes that were significantly different (False detection rate (FDR) $\leq 0.1$ and absolute fold-change $(F C) \geq 1.2$ ) in smaller versus larger healthy follicles of both HighVAR and LowVAR sows (middle), in smaller versus larger follicles of HighVAR sows, but not of LowVAR sows (left) and in smaller versus larger follicles of LowVAR sows, but not of HighVAR sows (right). NB Circles are not to scale. (B) Top 10 pathways using the 349 genes with a FDR $\leq 0.2$ and FC $\geq 1.2$ in small versus large follicles of both HighVAR and LowVAR sows, analysed by Reactome. For each pathway, the number of different genes, total number of genes and significance (FDR) are shown. (C) Significantly different genes of the glycolysis pathway (FDR = $1.87 E-3$, analysed by Reactome). For each gene, the fold-change (FC) and significance (FDR) are shown for both HighVAR and LowVAR sows. 
High cell proliferation rates are accompanied by a high demand for energy to facilitate e.g. DNA synthesis and chromosome segregation (Salazar-Roa and Malumbres, 2017). Therefore, we analysed pathways and individual transcripts known to be involved in energy metabolism. The most regulated metabolic pathway in granulosa cells of smaller follicles was glycolysis (FDR 1.87E-3). Individual transcript analysis of the genes in this pathway revealed an increased expression of phosphoglycerate kinase 1 (PGK1; HighVAR only), aldolase fructosebisphosphate C (ALDOC), glyceraldehyde-3-phosphate dehydrogenase (GAPDH), triosephosphate isomerase 1 (TPI1; HighVAR only), pyruvate kinase (PKM; HighVAR only) and several genes transcribing nucleoporins in the granulosa cells of smaller versus larger follicles (Fig. 3.3C). Of note, this set contains mRNAs for the rate limiting glycolysis proteins HK1 and PKM. On the protein level, two isoforms exist of PKM, constitutive PKM1 and regulated PKM2. To investigate possible upregulation of PKM2, we performed an immunostaining for PKM2, which showed a higher expression in smaller versus larger follicles for both LowVAR and HighVAR sows (Fig. 3.4B). Together these data indicate an increased glycolytic rate in granulosa cells of the smaller follicles. This is confirmed by a higher expression of LDHA (FC = 2.58 and FDR $=0.07$ for HighVAR and $F C=2.50$ and FDR $=0.10$ for LowVAR) and $\angle D H B(F C=$ 1.59 and FDR $=0.05$ for HighVAR and FC = 1.37 and FDR = 0.19 for LowVAR), which both encode lactate dehydrogenase (Supplemental Table S3.2). Increased expression of LDH may imply a shift from oxidative to glycolytic metabolism. Indeed, pathway analysis showed that, in contrast to increased glycolysis, oxidative phosphorylation (OXPHOS) was not differentially regulated. Furthermore, smaller follicles displayed a higher expression of fatty acid binding protein 3 (FABP3; FC $=2.90$ and FDR $=0.01$ for HighVAR and $F C=2.23$ and $F D R=0.07$ ) as compared to large follicles.
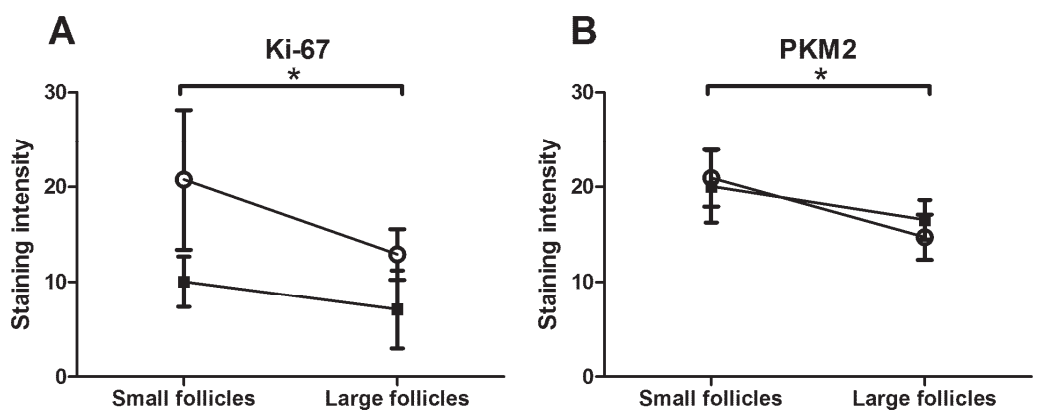

Figure 3.4 - Ki-67 and PKM2 protein levels in smaller versus larger follicles. (A) Ki-67 and (B) PKM2 protein expression in granulosa cells of the largest versus smallest follicle of the 10 largest healthy follicles within sows with high variation in follicle size (HighVAR) versus low variation (LowVAR). To determine protein expression, the mean staining intensity (sum of gray values in all pixels divided by the number of pixels selected) was measured in all visually intact parts of the granulosa cell layer. ${ }^{*} p$ $<0.05$. 
Subsequently, we analysed granulosa cell gene expression of paracrine growth factors, growth factor receptors and gonadotropin receptors, as they are of crucial importance in regulating granulosa cell proliferation. We analysed a total of 46 paracrine growth factors and receptors involved in follicular development according to the Ovarian Kaleidoscope Database (Hsueh and Rauch, 2012), of which 38 were included on the microarrays and 22 had detectable expression levels. In HighVAR sows, inhibin beta $\mathrm{b}(I N H B B)(F D R=0.02)$, androgen receptor $(A R)(F D R=0.02)$, epidermal growth factor receptor $(E G F R)(F D R=0.08)$ and anti-müllerian hormone $(A M H)(F D R=0.01)$ expression were higher in smaller follicles, while insulin-like growth factor 1 (IGF1) (FDR = 0.03) and angiopoietin 1 (ANGPT1) (FDR = 0.04) were significantly higher in larger follicles. These differences were not present in LowVAR sows (Fig. 3.5).

Finally, to study the granulosa cell processes that determine large differences in antral follicle size of follicles within the same antral follicle pool, we analysed the genes that were significantly different in HighVAR but not in LowVAR sows. A total of 570 unique genes were significantly different between smaller and larger follicles in HighVAR sows but were not differentially regulated in LowVAR (Fig. 3.6A; Supplemental Table S3.3). Pathway analysis using genes with a FDR $\leq 0.2$ and $F C \geq 1.2$ showed that 7 of the 10 top pathways were involved in protein translation (Fig. 3.6B). We additionally performed a more stringent pathway analysis by only including genes with a FDR $\leq 0.1$ and identified similar processes (see Supplemental Table S3.4). Individual transcript analysis of the genes involved in these pathways showed an increased expression of genes encoding for ribosomal proteins (e.g. RPs and RLs) in smaller versus larger follicles (genes with highest absolute FC are shown in Fig. 3.6C). The top genes that were significantly different in LowVAR sows but not in HighVAR sows are depicted in Supplemental Table S3.5. 


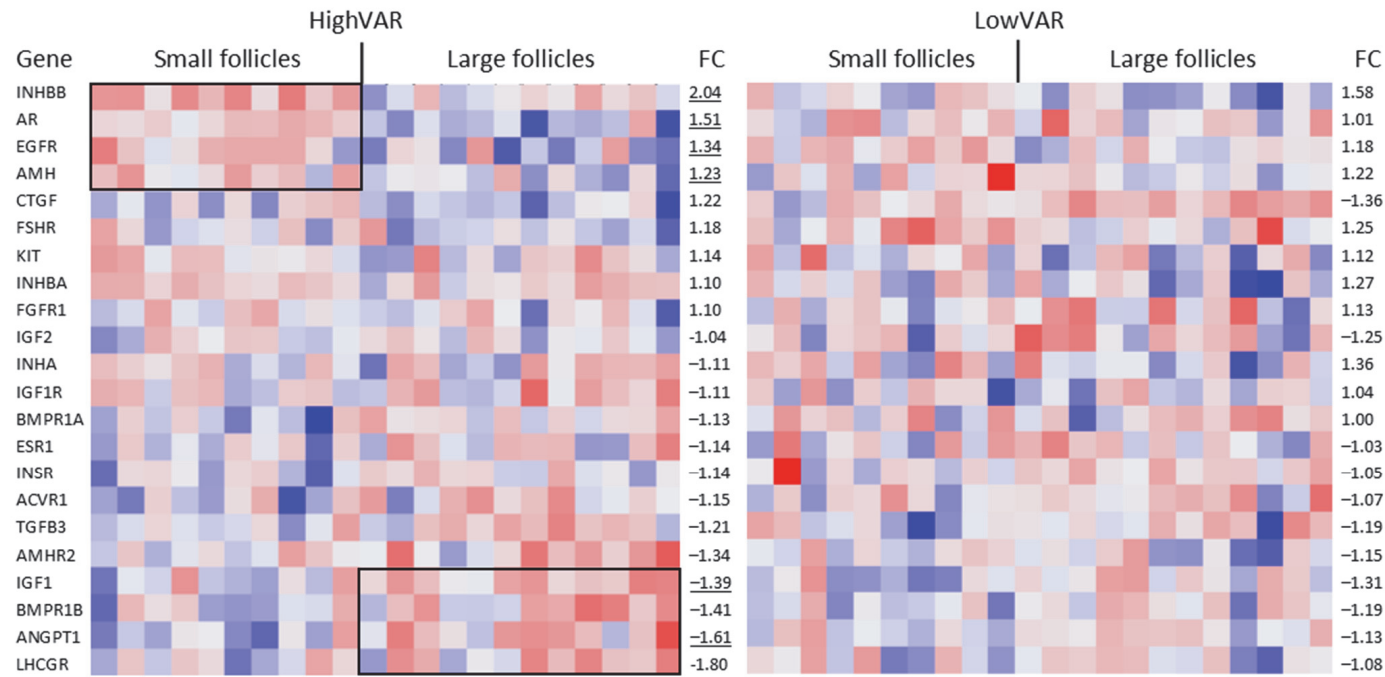

Figure 3.5 - Paracrine growth factors and receptors in smaller versus larger follicles. Heat map representing the mean-centered log2 expression of paracrine growth factors and receptors in granulosa cells of smaller and larger follicles from sows with high variation in follicle size (HighVAR; left) versus low variation (LowVAR right). Red indicates a high relative expression and blue indicates a low relative expression. The 22 presented genes are selected from all known granulosa cell paracrine growth factors and receptors which are involved in follicular development according to the Ovarian Kaleidoscope Database (Hsueh and Rauch, 2012), of which probes were present on the microarrays (38/46) and which had average expression levels that exceeded background levels over 2 times (22/46). For both HighVAR and LowVAR sows, the fold-change (FC) of smaller versus larger follicles is shown. If more than 2 probes of the same gene were present on the microarrays, we averaged the FC. Significant genes $(F D R<0.1)$ are underlined. 


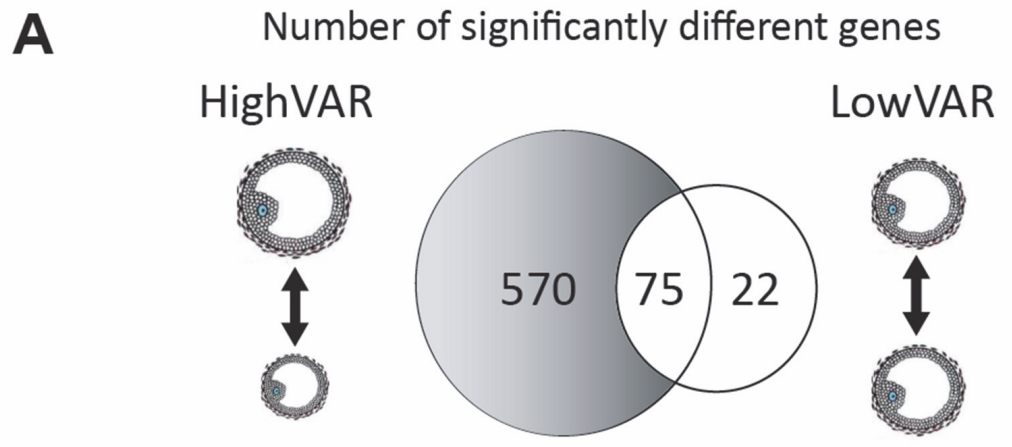

$\begin{array}{lllll}\mathbf{B} & \text { Pathway } & \text { Genes } & \text { Total } & \text { FDR } \\ \mathbf{1} & \text { Regulation of expression of SLITs and ROBOs } & 36 & 183 & 4.08 \mathrm{E}-04 \\ \mathbf{2} & \text { Nonsense Mediated Decay (NMD) independent of the Exon Junction Complex (EJC) } & 25 & 101 & 4.08 \mathrm{E}-04 \\ \mathbf{3} & \text { SRP-dependent co-translational protein targeting to membrane } & 27 & 121 & 6.59 \mathrm{E}-04 \\ \mathbf{4} & \text { Eukaryotic Translation Termination } & 25 & 108 & 6.59 \mathrm{E}-04 \\ \mathbf{5} & \text { Formation of a pool of free 40S subunits } & 24 & 106 & 1.05 \mathrm{E}-03 \\ \mathbf{6} & \text { Peptide chain elongation } & 23 & 99 & 1.05 \mathrm{E}-03 \\ \mathbf{7} & \text { L13a-mediated translational silencing of Ceruloplasmin expression } & 25 & 118 & 1.72 \mathrm{E}-03 \\ \mathbf{8} & \text { Eukaryotic Translation Elongation } & 23 & 104 & 1.72 \mathrm{E}-03 \\ \mathbf{9} & \text { Signaling by ROBO receptors } & 39 & 235 & 1.72 \mathrm{E}-03 \\ 10 & \text { GTP hydrolysis and joining of the 60S ribosomal subunit } & 25 & 122 & 1.89 \mathrm{E}-03\end{array}$

$\begin{array}{ll}\text { Gene symbol } & \text { Protein translation } \\ \text { Gene name } \\ \text { RPN1 } & \text { Ribophorin 1 } \\ \text { RPL12 } & \text { Ribosomal protein L12 } \\ \text { RPL8 } & \text { Ribosomal protein L8 } \\ \text { RPL6 } & \text { Ribosomal protein L6 } \\ \text { RPS12 } & \text { Ribosomal protein S12 } \\ \text { RPN2 } & \text { Ribophorin 2 } \\ \text { RPL27 } & \text { Ribosomal protein L27 } \\ \text { RPS26 } & \text { Ribosomal protein S26 } \\ \text { RPL19 } & \text { Riosomal protein L19 } \\ \text { RPL14 } & \text { Ribosomal protein L14 }\end{array}$

\begin{tabular}{lll} 
& \multicolumn{2}{c}{ HighVAR } \\
Sequence ID & FC & FDR \\
NM_214333 & 1.49 & 0.01 \\
XM_005660442 & 1.48 & 0.03 \\
XM_005655297 & 1.47 & 0.07 \\
NM_001044542 & 1.45 & 0.07 \\
NM_214363 & 1.40 & 0.09 \\
NM_213941 & 1.38 & 0.09 \\
NM_001097479 & 1.37 & 0.10 \\
NM_001097481 & 1.35 & 0.10 \\
XM_003131509 & 1.35 & 0.09 \\
NM_001097478 & 1.35 & 0.10
\end{tabular}

Figure 3.6 - Gene expression in smaller versus larger follicles of sows with high variation in follicle size (HighVAR). (A) The Venn diagram showing the number of genes that were significantly different (false detection rate (FDR) $\leq 0.1$ and absolute fold-change $\geq 1.2$ ) in smaller versus larger healthy follicles of both HighVAR and LowVAR sows (middle), in smaller versus larger follicles of HighVAR sows but not of LowVAR sows (left) and in smaller versus larger follicles of LowVAR sows, but not of HighVAR sows (right). (B) Top 10 pathways using the 821 genes with a FDR $\leq 0.2$ and FC $\geq 1.2$ in smaller versus larger follicles of HighVAR sows only, analysed by Reactome. For each pathway, the number of different genes, total number of genes and significance (FDR) are shown. (C) Most regulated genes involved in protein translation. The fold-change (FC) of smaller versus larger follicles and significance (FDR) are shown. 


\section{Discussion}

One of the major findings is that both gene and protein expression analysis revealed increased cell proliferation in smaller versus larger follicles at the start of the follicular phase. This was shown by pathway analysis as well as individual transcript analysis, which showed an increased expression of genes regulating cell cycle progression in smaller follicles. In addition, smaller follicles showed elevated expression of the nuclear antigen Ki-67, a marker for proliferating cells (Gerdes et al., 1984). These findings indicate that granulosa cells of smaller follicles have a higher proliferation rate as compared to the larger follicles in the same pool, which can facilitate follicular growth in the smaller follicles.

To investigate this difference in granulosa cell proliferation between the smaller and larger follicles further, we analysed expression of paracrine growth factors, growth factor receptors and gonadotropin receptors, as these growth factors and gonadotropins can stimulate granulosa cell proliferation in a time and species dependent manner (Juengel and McNatty, 2005). These growth factors include members of the transforming growth factor $\beta$ (TGF $\beta$ ) superfamily. This family includes oocyte secreted factors (OSFs) e.g. growth differentiation factors (GDFs) and bone morphogenetic proteins (BMPs), potent mitogens which mainly signal via granulosa cell transforming growth factor beta receptor 2 (TGFBR2) (Gilchrist et al., 2006). By binding to their associated receptor, OSFs can stimulate granulosa cell proliferation by inducing the expression of cyclin D2 (Gilchrist et al., 2008). Indeed, in our study, smaller follicles of HighVAR sows tended to exhibit higher levels of cyclin D2 ( $F C=1.57$ and FDR $=0.04$ ), which confirms high granulosa cell proliferation rates in smaller follicles. Unfortunately, we were unable to study granulosa cell TGBR2 expression as expression levels did not exceed background levels. Next to GDFs and BMPs, EGF and related growth factors play a fundamental role in regulating granulosa cell proliferation. EGF-like growth factors bind to EGFR, and inhibition of EGFR resulted in decreased granulosa cell proliferation rates in mice secondary follicles and antral follicles (Sasseville et al., 2010). In addition, results of Sasseville et al. (2010) indicate that granulosa cell receptivity for mitogenic actions of OSFs is dependent on EGFR activation. Results of our study confirm that EGFR is indeed essential for upregulation of granulosa cell proliferation, as the more proliferative smaller follicles had a higher expression of EGFR. Similarly, $A R$ was upregulated in smaller follicles. Previous studies indicate that androgen receptor ligands (testosterone, dihydro-testosterone) increase OSF induced porcine mural granulosa cell proliferation, indicating an essential role for AR in this process (Hickey et al., 2005). Therefore, results of our study indicate that the upregulation of both $E G F R$ and $A R$ in smaller follicles, might be crucial in regulating granulosa cell proliferation.

Granulosa cell proliferation is also regulated by the pituitary produced gonadotropins, follicle stimulating hormone (FSH) and luteinizing hormone (LH). Depending on the animal species, from the late pre-antral/small antral follicle stage, follicles become critically dependent on FSH for their growth as is shown by an FSH-induced increase in the expression of the 
proliferation marker PCNA (Yu et al., 2005). Despite the fact that the granulosa cells of smaller and larger follicles in the present study showed similar expression of FSHR, PCNA expression was higher in smaller follicles for both HighVAR and LowVAR sows (FC $=1.89, F D R=0.03)$. As antral follicular development continues, porcine granulosa cells under the influence of FSH, acquire $L H C G R$, which enables $L H$ to induce terminal differentiation and inhibit proliferation of granulosa cells (Robker and Richards, 1998). Surprisingly, we found that LHCGR was already expressed in the granulosa cells at the onset of the follicular phase, when follicles have yet to be selected to grow and develop into pre-ovulatory follicles. LHCGR could therefore play an important role in regulating granulosa cell proliferation and subsequent follicular growth already from the onset of the follicular phase onwards. This in contrast to what is found in rats and mice, where LHCGR is only expressed in granulosa cells at a much later developmental stage, namely, from pre-ovulatory follicles onwards (Peng et al.,1991; Teerds and Dorrington, 1995). The LHCGR expression pattern of sows therefore resembles the human situation, where granulosa cell LHCGR is expressed in antral follicles throughout the follicular phase of the menstrual cycle (Yung et al., 2014). Our findings therefore reveal that follicular development of sows shows similarities to human follicular development. Similarities between human and sow follicular development were also found in studies of AMH expression during follicular development. AMH is exclusively expressed in granulosa cells in all studied animal species, except for humans and pigs, in which $\mathrm{AMH}$ is also expressed in theca cells of pre-ovulatory follicles (Almeida et al., 2018; Stubbs et al., 2005). These similarities provide evidence that pigs could serve as a suitable animal model to study human follicular development.

In the HighVAR sows IGF1 expression was higher in larger follicles. IGF1 can synergise with LH to activate LH target genes (Rawan et al., 2015), with only cytochrome P450 family 11 subfamily A member 1 (CYP11A1) being upregulated (HighVAR only, FDR $=0.04$ ) in larger follicles of the current study. This higher level of IGF1 might result in more LH-induced inhibition of cell proliferation, thereby explaining the lower proliferation rate in larger follicles. In addition, since granulosa cell IGF1 is considered a follicular maturation marker in pigs and cows (May and Schomberg, 1984; Spicer et al., 1988), high expression levels, as we observed in the larger follicles, might indicate that the larger follicles have already initiated terminal differentiation. This hypothesis is supported by the higher expression of ANGPT1 in larger follicles. ANGPT1 is an angiogenic factor that is necessary for blood vessel maturation (Robinson et al., 2009) and increases in granulosa cells in late stages of follicular development just before ovulation and corpus luteum formation (Hayashi et al., 2003; Hazzard et al., 1999). Together, these results indicate that smaller follicles in the healthy antral follicle pool are in a more proliferative state, while the larger follicles in the pool are more differentiated, and that these differences are already present at the start of the follicular phase, reflecting the heterogeneity of this follicle pool. 
High levels of cell proliferation, as seen in especially the smaller follicles of the current study, are accompanied by high energy demands. To facilitate these energy demands major changes in metabolic pathways such as OXPHOS and glycolysis are needed, which is exemplified by many nutrient-sensitive restriction points in the cell cycle (Salazar-Roa and Malumbres, 2017). Indeed, in our study, pathway analysis showed upregulation of glycolysis in the highly proliferating granulosa cells of the smaller follicles while OXPHOS was not differentially regulated. In addition, we found higher expression of $L D H$ and these results together might imply a shift from oxidative to glycolytic metabolism. Granulosa cells of growing follicles thereby seem to resemble the metabolic profile of highly proliferating cells such as stimulated immune cells, cancer cells and in pre-implantation embryonic cells (Krisher and Prather, 2012). In contrast to normal differentiated cells, which rely primarily on mitochondrial oxidative phosphorylation to produce ATP, most high proliferating cells rely on aerobic glycolysis, i.e. display the Warburg effect (Vander Heiden et al., 2009). Proliferating cells exhibiting this metabolic profile preferentially express the M2 isoform of pyruvate kinase (PKM2) (Yang et al., 2012). In our study, PKM2 protein expression was indeed higher in smaller versus larger follicles. This preferential expression of PKM2 provides a metabolic advantage for highly proliferating cells, as PKM2 can be inactivated into its dimeric form by phosphorylation at tyrosine105. This inactivation of PKM2 prevents the conversion of glycolytic intermediates into pyruvate, thereby inhibiting oxidative phosphorylation. This finally results in a build-up of glycolytic intermediates and the channelling into pentose phosphate pathway as well as phospholipid, nucleic acid and amino acid synthesis to support cell proliferation (Vander Heiden et al., 2009). In addition, PKM2 can localize in the nucleus via several posttranslational modifications, to facilitate gene expression of e.g. GLUT1, cyclins, LDHA and PKM itself to support increased glucose uptake, cell proliferation and lactate production in proliferating cells (Prakasam et al., 2018). In our study, we found increased expression of the PKM2 regulated genes, $L D H A$, several cyclins and PKM itself. These findings support our hypothesis that proliferating granulosa cells exhibit the Warburg effect, which could enable the follicles to maintain a high growth rate by providing the necessary energy and building-blocks needed for granulosa cell proliferation, facilitating follicle growth.

Finally, we studied the pathways of genes that were only differently expressed between the smaller and larger follicles in the HighVAR sows but were not differentially expressed in the LowVAR sows. In HighVAR sows, the difference in follicle size between smaller and larger follicles (3.8 versus $6.9 \mathrm{~mm}$ ) was larger compared to the difference in LowVAR sows (4.3 versus $6.1 \mathrm{~mm}$ ), which might have resulted in the expression of additional significant genes. Pathway analysis of the 821 genes (FDR $\leq 0.2$ and FC $\geq 1.2$ ) showed that granulosa cells of smaller follicles highly expressed genes of pathways related to protein translation. This increased protein translation might be a necessary adaptive mechanism to support the very high proliferation rates in the smaller antral follicles of the HighVAR sows that were analysed in this study. 
To summarize, we performed an in vivo study to determine granulosa cell processes at the start of the follicular phase, so 5-6 days before ovulation, which correlate with antral follicle size. We specifically investigated the healthy follicles of the presumed ovulatory pool of which we isolated pure mural granulosa cells. Granulosa cells of the smaller antral follicles showed more cell proliferation, which was accompanied by a metabolic shift towards aerobic glycolysis similar to other highly proliferating cells. High granulosa cell proliferation rates in smaller follicles might be regulated via increased expression of receptors for locally produced mitogens. While granulosa cells of smaller follicles were more proliferative, which is indicative of higher follicular growth, granulosa cells of larger follicles showed less proliferation and were more differentiated, as they showed a higher expression of granulosa cell maturation markers.

\section{Implications}

IVF treatment includes administration of supra-physiological doses of FSH to induce growth of multiple ovarian follicles, after which a trigger is administered to induce oocyte maturation. By applying this controlled ovarian hyperstimulation, not only the largest but also smaller follicles in the antral follicle pool can be recruited and selected to develop into pre-ovulatory follicles, after which these follicles are aspirated for oocyte retrieval. However, consequences of aspiration and oocyte retrieval of these smaller follicles on IVF outcomes are not clear, as some studies find positive associations between follicle size and IVF outcome (Schumacher et al., 2018), while others do not (Knopman et al., 2012). Similarly, some studies in humans and animal models find direct relations between antral follicle size and oocyte quality; where larger antral follicles yielded better quality oocytes with increased blastocyst formation and implantation rates (Algriany et al., 2004; Ectors et al., 1997; Lequarre et al., 2005), while other studies do not find these relations (Pavlok et al., 1992; Seneda et al., 2001). Results of our study in which we analysed pure samples of mural granulosa cells in different-sized follicles of ovaries which were all in the same developmental stage, indicate that large differences between smaller and larger follicles in the healthy antral follicle pool already exist at the start of the follicular phase, as smaller follicles still show high levels of granulosa cell proliferation for follicle growth, while larger follicles are already differentiated into more mature follicles. This is exemplified by the higher ANGPT1 expression, which improves vascularity, and higher IGF1 expression in granulosa cells of larger follicles, which have both been associated with improved human IVF outcomes (Faraj et al., 2017; Vural et al., 2016). This implies that including smaller follicles for oocyte aspiration might have negative consequences for IVF outcome. Therefore, our results might imply that the inclusion of strict criteria of antral follicle size in IVF protocols could improve IVF outcome.

\section{Acknowledgements}

We acknowledge Wageningen Institute for Animal Sciences (WIAS) and NWO for providing the NWO-WIAS Graduate Programme 2015 grant. 


\section{References}

Algriany, O., Bevers, M., Schoevers, E., Colenbrander, B., \& Dieleman, S. (2004). Follicle size-dependent effects of sow follicular fluid on in vitro cumulus expansion, nuclear maturation and blastocyst formation of sow cumulus oocytes complexes. Theriogenology, 62(8), 1483-1497.

Almeida, F. R., Costermans, N. G. J., Soede, N. M., Bunschoten, A., Keijer, J., Kemp, B., \& Teerds, K. J. (2018). Presence of anti-Müllerian hormone (AMH) during follicular development in the porcine ovary. PLoS ONE, 13(7), e0197894.

Carpintero, N. L., Suárez, O. A., Varea, C. G., Rioja, R. G., \& Mangas, C. C. (2015). Variability between the follicular steroid hormone levels in different follicles of the same patient and between patients. Journal of human reproductive sciences, 8(1), 37-42.

Christianson, M. S., Shoham, G., Tobler, K. J., Zhao, Y., Cordeiro, C. N., Leong, M., \& Shoham, Z. (2015). Measurement of antral follicle count in patients undergoing in vitro fertilization treatment: results of a worldwide web-based survey. Journal of assisted reproduction and genetics, 32(10), 1435-1440.

Costermans, N. G. J., Teerds, K. J., Keijer, J., Knol, E. F., Koopmanschap, R. E., Kemp, B., \& Soede, N. M. (2019). Follicular development of sows at weaning in relation to estimated breeding value for within-litter variation in piglet birth weight. Animal, 13(3), 554-563.

da Silva, C. L. A., Laurenssen, B. F. A., Knol, E. F., Kemp, B., \& Soede, N. M.(2016). Relationships between ovulation rate and embryonic and placental characteristics in multiparous sows at 35 days of pregnancy. Animal, 10(7), 1192-1199.

Ectors, F. J., Vanderzwalmen, P., Van Hoeck, J., Nijs, M., Verhaegen, G., Delvigne, A., Shoysman, R. \& Leroy, F. (1997). Relationship of human follicular diameter with oocyte fertilization and development after in-vitro fertilization or intracytoplasmic sperm injection. Human reproduction, 12(9), 2002-2005.

Faraj, N., Alhalabi, M., \& Al-Quobaili, F. (2017). Predictive value of follicular fluid insulin like growth factor-1 in IVF outcome of normo-ovulatory women. Middle east fertility society journal, 22(2), 101-104.

Field, L. A., Deyarmin, B., Shriver, C. D., Ellsworth, D. L., \& Ellsworth, R. E. (2011). "Laser microdissection for gene expression profiling." In Laser capture microdissection (pp. 17-45). Humana Press: Totowa, NJ, US.

Foxcroft, G. R., Shaw, H. J., Hunter, M. G., Booth, P. J., \& Lancaster, R. T. (1987). Relationships between luteinizing hormone, follicle-stimulating hormone and prolactin secretion and ovarian follicular development in the weaned sow. Biology of reproduction, 36(1), 175-191.

Gerdes, J., Lemke, H., Baisch, H., Wacker, H.-H., Schwab, U., \& Stein, H. (1984). Cell cycle analysis of a cell proliferation-associated human nuclear antigen defined by the monoclonal antibody Ki67. The journal of immunology, 133(4), 1710-1715.

Gilchrist, R. B., Lane, M., \& Thompson, J. G. (2008). Oocyte-secreted factors: regulators of cumulus cell function and oocyte quality. Human reproduction update, 14(2), 159-177.

Gilchrist, R. B., Ritter, L. J., Myllymaa, S., Kaivo-Oja, N., Dragovic, R. A., Hickey, T. E., Ritvos, O., \& Mottershead, D. G. (2006). Molecular basis of oocyte-paracrine signalling that promotes granulosa cell proliferation. Journal of cell science, 119(18), 3811-3821.

Hayashi, K.-G., Acosta, T. J., Tetsuka, M., Berisha, B., Matsui, M., Schams, D., Ohtani, M., \& Miyamoto, A. (2003). Involvement of angiopoietin-tie system in bovine follicular development and atresia: messenger RNA expression in theca interna and effect on steroid secretion. Biology of reproduction, 69(6), 2078-2084.

Hazzard, T. M., Molskness, T. A., Chaffin, C. L., \& Stouffer, R. L. (1999). Vascular endothelial growth factor (VEGF) and angiopoietin regulation by gonadotrophin and steroids in macaque granulosa cells during the peri-ovulatory interval. Molecular human reproduction, 5(12), 11151121. 
Hickey, T. E., Marrocco, D. L., Amato, F., Ritter, L. J., Norman, R. J., Gilchrist, R. B., \& Armstrong, D. T. (2005). Androgens augment the mitogenic effects of oocyte-secreted factors and growth differentiation factor 9 on porcine granulosa cells. Biology of reproduction, 73(4), 825-832.

Hoek-van den Hil, E. F., Keijer, J., Bunschoten, A., Vervoort, J. J. M., Stankova, B., Bekkenkamp, M., Herreman, L., Venema, D., Hollman, P. C. H. Tvrzicka, E., \& van Schothorst E. M. (2013). Quercetin induces hepatic lipid omega-oxidation and lowers serum lipid levels in mice. PLoS ONE, 8(1), e51588.

Hsueh, A. J., \& Rauch, R. (2012). Ovarian Kaleidoscope database: ten years and beyond. Biology of reproduction, 86(6), 192, 191-197.

Hunter, M. G., \& Wiesak, T. (1990). Evidence for and implications of follicular heterogeneity in pigs. Journal of reproduction and fertility. Supplement, 40, 163-177.

Spicer, L. J., Echternkamp, S. E., Canning, S. F., \& Hammond. (1988). Relationship between concentrations of immunoreactive insulin-like growth factor-I in follicular fluid and various biochemical markers of differentiation in bovine antral follicles. Biology of reproduction, 39(3), 573-580.

Jiang, J.-Y., Xiong, H., Cao, M., Xia, X., Sirard, M.-A., \& Tsang, B. K. (2010). Mural granulosa cell gene expression associated with oocyte developmental competence. Journal of ovarian research, $3(1), 6$.

Juengel, J. L., \& McNatty, K. P. (2005). The role of proteins of the transforming growth factor- $\beta$ superfamily in the intraovarian regulation of follicular development. Human reproduction update, 11(2), 144-161.

Knopman, J. M., Grifo, J. A., Novetsky, A. P., Smith, M. B., \& Berkeley, A. S. (2012). Is bigger better: the association between follicle size and livebirth rate following IVF. Fertility and sterility, 94(4), 28-29

Krisher, R. L., \& Prather, R. S. (2012). A role for the Warburg effect in preimplantation embryo development: metabolic modification to support rapid cell proliferation. Molecular reproduction and development, 79(5), 311-320.

Lai, Q., Chen, C., Zhang, Z., Zhang, S., Yu, Q., Yang, P., Hu, J., \& Wang, C.-Y. (2013). The significance of antral follicle size prior to stimulation in predicting ovarian response in a multiple dose $\mathrm{GnRH}$ antagonist protocol. International journal of clinical and experimental pathology, 6(2), 258266.

Lequarre, A.-S., Vigneron, C., Ribaucour, F., Holm, P., Donnay, I., Dalbies-Tran, R., Callesen, H., \& Mermillod, P. (2005). Influence of antral follicle size on oocyte characteristics and embryo development in the bovine. Theriogenology, 63(3), 841-859.

Matsuda, F., Inoue, N., Manabe, N., \& Ohkura, S. (2012). Follicular growth and atresia in mammalian ovaries: regulation by survival and death of granulosa cells. Journal of reproduction and development, 58(1), 44-50.

May, J. V., \& Schomberg, D. W. (1984). Developmental coordination of luteinizing hormone/human chorionic gonadotropin ( $\mathrm{hCG}$ ) receptors and acute $\mathrm{hCG}$ responsiveness in cultured and freshly harvested porcine granulosa cells. Endocrinology, 114(1), 153-163.

Moslehi, N., Mirmiran, P., Tehrani, F. R., \& Azizi, F. (2017). Current evidence on associations of nutritional factors with ovarian reserve and timing of menopause: a systematic review. Advances in nutrition, 8(4), 597-612.

Oktem, O., \& Urman, B. (2010). Understanding follicle growth in vivo. Human reproduction, 25(12), 2944-2954.

Pavlok, A. I., Lucas-Hahn, A., \& Niemann, H. (1992). Fertilization and developmental competence of bovine oocytes derived from different categories of antral follicles. Molecular reproduction and development, 31(1), 63-67.

Peng, X.-R., Hsueh, A. J. W., Lapolt, P. S., Bjersing, L., \& Ny, T. (1991). Localization of luteinizing hormone receptor messenger ribonucleic acid expression in ovarian cell types during follicle development and ovulation. Endocrinology, 129(6), 3200-3207. 
Prakasam, G., lqbal, M. A., Bamezai, R. N. K., \& Mazurek, S. (2018). Posttranslational Modifications of Pyruvate Kinase M2: Tweaks that Benefit Cancer. Frontiers in oncology, 8, 22.

Rawan, A. F., Yoshioka, S., Abe, H., \& Acosta, T. J. (2015). Insulin-Like Growth Factor-1 Regulates the Expression of Luteinizing Hormone Receptor and Steroid Production in Bovine Granulosa Cells. Reproduction in domestic animals, 50(2), 283-291.

Robinson, R. S., Woad, K. J., Hammond, A. J., Laird, M., Hunter, M. G., \& Mann, G. E. (2009). Angiogenesis and vascular function in the ovary. Reproduction, 138(6), 869-881.

Robker, R. L., \& Richards, J. S. (1998). Hormone-induced proliferation and differentiation of granulosa cells: a coordinated balance of the cell cycle regulators cyclin D2 and p27Kip1. Molecular endocrinology, 12(7), 924-940.

Salazar-Roa, M., \& Malumbres, M. (2017). Fueling the cell division cycle. Trends in cell biology, 27(1), 69-81.

Santamaría, D., Barrière, C., Cerqueira, A., Hunt, S., Tardy, C., Newton, K., Cáceres, J. F., Dubus, P., Malumbres, M., \& Barbacid, M. (2007). Cdk1 is sufficient to drive the mammalian cell cycle. Nature, 448(7155), 811.

Sasseville, M., Ritter, L. J., Nguyen, T. M., Liu, F., Mottershead, D. G., Russell, D. L., \& Gilchrist, R. B. (2010). Growth differentiation factor 9 signaling requires ERK1/2 activity in mouse granulosa and cumulus cells. Journal of cell science, 123(18): 3166-3176.

Schumacher, B. M. L., Mersereau, J. E., \& Steiner, A. Z. (2018). Cycle day, estrogen level, and lead follicle size: analysis of 27,790 in vitro fertilization cycles to determine optimal start criteria for gonadotropin-releasing hormone antagonist. Fertility and sterility, 109(4), 633-637.

Seneda, M. M., Esper, C. R., Garcia, J. M., de Oliveira, J. A., \& Vantini, R. (2001). Relationship between follicle size and ultrasound-guided transvaginal oocyte recovery. Animal reproduction science, 67(1-2), 37-43.

Soede, N. M., Langendijk, P., \& Kemp, B. (2011). Reproductive cycles in pigs. Animal reproduction science, 124(3), 251-258.

Stubbs, S. A., Hardy, K., Da Silva-Buttkus, P., Stark, J., Webber, L. J., Flanagan, A. M., Themmen, A. P., Visser, J. A., Groome, N. P., \& Franks, S. (2005). Anti-mullerian hormone protein expression is reduced during the initial stages of follicle development in human polycystic ovaries. The journal of clinical endocrinology \& metabolism, 90(10), 5536-5543.

Sugiura, K., Pendola, F. L., \& Eppig, J. J. (2005). Oocyte control of metabolic cooperativity between oocytes and companion granulosa cells: energy metabolism. Developmental biology, 279(1), 20-30.

Svotelis, A., Gévry, N., Grondin, G., \& Gaudreau, L. (2010). H2A. Z overexpression promotes cellular proliferation of breast cancer cells. Cell cycle, 9(2), 364-370.

Teerds, K. J., \& Dorrington, J. H. (1995). Immunolocalization of transforming growth factor a and luteinizing hormone receptor in healthy and atretic follicles of the adult rat ovary. Biology of reproduction, 52(3), 500-508.

van Schothorst, E. M., Pagmantidis, V., de Boer, V. C., Hesketh, J., \& Keijer, J. (2007). Assessment of reducing RNA input for Agilent oligo microarrays. Analytical biochemistry, 363(2), 315-317.

Vanden Brink, H., Robertson, D. M., Lim, H., Lee, C., Chizen, D., Harris, G., Hale, G., Burger, H., \& Baerwald, A. (2015). Associations between antral ovarian follicle dynamics and hormone production throughout the menstrual cycle as women age. The journal of clinical endocrinology \& metabolism, 100(12), 4553-4562.

Vander Heiden, M. G., Cantley, L. C., \& Thompson, C. B. (2009). Understanding the Warburg effect: the metabolic requirements of cell proliferation. Science, 324(5930), 1029-1033.

Vural, F., Vural, B., Doğer, E., Çakıroğlu, Y., \& Çekmen, M. (2016). Perifollicular blood flow and its relationship with endometrial vascularity, follicular fluid EG-VEGF, IGF-1, and inhibin-a levels and IVF outcomes. Journal of assisted reproduction and genetics, 33(10), 1355-1362.

Yang, W., Zheng, Y., Xia, Y., Ji, H., Chen, X., Guo, F., Lyssiotis, C. A., Aldape, K., Cantley, L. C., \& Lu, Z. (2012). ERK1/2-dependent phosphorylation and nuclear translocation of PKM2 promotes the Warburg effect. Nature cell biology, 14(12), 1295. 
Yu, F.-Q., Han, C.-S., Yang, W., Jin, X., Hu, Z.-Y., \& Liu, Y.-X. (2005). Role of ERK1/2 in FSH induced PCNA expression and steroidogenesis in granulosa cells. Frontiers in bioscience, 10(1-3), 896-904.

Yung, Y., Aviel-Ronen, S., Maman, E., Rubinstein, N., Avivi, C., Orvieto, R., \& Hourvitz, A. (2014). Localization of luteinizing hormone receptor protein in the human ovary. Molecular human reproduction, 20(9), 844-849.

\section{Supplemental Material}

Supplemental Table S3.1 - Top 10 pathways differently expressed in smaller versus larger follicles of both HighVAR and LowVAR sows, using false discovery rate (FDR) $\leq 0.10$ genes.

\begin{tabular}{llccc} 
& Pathway $^{1}$ & Number $^{2}$ & Total $^{3}$ & FDR \\
\hline $\mathbf{1}$ & Cell Cycle, Mitotic & 19 & 570 & $3.68 \mathrm{E}-5$ \\
$\mathbf{2}$ & Cell Cycle & 20 & 682 & $6.16 \mathrm{E}-5$ \\
$\mathbf{3}$ & TFAP2A acts as transcriptional repressor during retinoic acid & 4 & 9 & $1.76 \mathrm{E}-4$ \\
& $\begin{array}{l}\text { induced cell differentiation } \\
\mathbf{4}\end{array}$ & & & \\
$\mathbf{5}$ & Mitotic G1-G1/S phases & 9 & 173 & 0.001 \\
$\mathbf{6}$ & Transcription of E2F targets & 4 & 20 & 0.002 \\
$\mathbf{7}$ & The role of GTSE1 in G2/M progression after G2 checkpoint & 6 & 83 & 0.005 \\
$\mathbf{8}$ & Organelle biogenesis and maintenance & 11 & 335 & 0.005 \\
$\mathbf{9}$ & G1/S Transition & 7 & 150 & 0.012 \\
$\mathbf{1 0}$ & DNA strand elongation & 7 & 38 & 0.012 \\
\hline
\end{tabular}

${ }^{1}$ Pathway analysis was performed using Reactome, ${ }^{2}$ Number of significantly different genes in the pathway,

${ }^{3}$ Total number of genes in the pathway. 
Supplemental Table S3.2 - Top 20 false discovery rate (FDR) $\leq 0.1$ genes differing mostly in foldchange (FC) between smaller vs. larger follicles of HighVAR and LowVAR sows.

\begin{tabular}{|c|c|c|c|c|c|c|}
\hline \multirow[b]{2}{*}{ Gene symbol } & \multirow[b]{2}{*}{ Gene description } & \multirow[b]{2}{*}{ Accession $\mathrm{nr}$} & \multicolumn{2}{|c|}{ Log2 expression } & \multirow[b]{2}{*}{ FC } & \multirow[b]{2}{*}{ FDR } \\
\hline & & & $\begin{array}{c}\text { Small } \\
\text { follicles }\end{array}$ & $\begin{array}{c}\text { Large } \\
\text { follicles }\end{array}$ & & \\
\hline LOC100154071 & Histone $\mathrm{H} 2 \mathrm{~A}$ type 1-like & - & 10.06 & 8.53 & 2.89 & 0.02 \\
\hline MGST1 & Microsomal glutathione S-transferase 1 & NM_214300 & 7.72 & 9.19 & -2.78 & 0.02 \\
\hline LOC595122 & Histone H1.3-like protein & - & 11.49 & 10.06 & 2.69 & 0.01 \\
\hline ADGRG1 & Adhesion G protein-coupled receptor & NM_001243056 & 13.04 & 11.72 & 2.50 & 0.04 \\
\hline ENSSSCT00000023491 & Unknown & - & 9.96 & 8.74 & 2.32 & 0.01 \\
\hline LOC100153329 & Histone $\mathrm{H} 2 \mathrm{~A}$ type $1-\mathrm{A}$ & - & 8.77 & 7.62 & 2.21 & 0.02 \\
\hline ENSSSCT00000006901 & Unknown & - & 5.79 & 6.93 & -2.21 & 0.01 \\
\hline AMY2 & Amylase, alpha 2B & NM_214195 & 7.24 & 8.37 & -2.19 & 0.03 \\
\hline LOC574051 & Histone H1.2-like protein & - & 10.32 & 9.19 & 2.18 & 0.02 \\
\hline ENSSSCT00000011432 & Unknown & - & 10.37 & 9.26 & 2.16 & 0.05 \\
\hline MT1A & Metallothionein $1 \mathrm{~A}$ & NM_001001266 & 7.19 & 6.10 & 2.14 & 0.04 \\
\hline DAPL1 & Death associated protein like 1 & NM_001185174 & 9.36 & 10.45 & -2.12 & 0.06 \\
\hline NABP1 & Nucleic acid binding protein 1 & NM_001244491 & 7.14 & 8.20 & -2.08 & 0.04 \\
\hline ENSSSCT00000001263 & Unknown & - & 9.34 & 8.30 & 2.06 & 0.01 \\
\hline RBP1 & Retinol binding protein 1 & NM_001031789 & 8.98 & 7.94 & 2.06 & 0.02 \\
\hline INHBB & Inhibin subunit beta B & NM_001164842 & 11.38 & 10.35 & 2.04 & 0.02 \\
\hline ENSSSCT00000017182 & Unknown & - & 6.88 & 7.86 & -1.98 & 0.02 \\
\hline ATP1B1 & ATPase $\mathrm{Na}+/ \mathrm{K}+$ transporting subunit & NM_001001542 & 6.81 & 7.80 & -1.98 & 0.05 \\
\hline TXNIP & Thioredoxin interacting protein & NM_001044614 & 8.89 & 9.85 & -1.95 & 0.01 \\
\hline HMGB2 & High mobility group box 2 & NM_214063 & 9.31 & 8.36 & 1.94 & 0.06 \\
\hline
\end{tabular}


Supplemental Table S3.3 - Top 20 false discovery rate (FDR) $\leq 0.1$ genes differing mostly in foldchange (FC) between smaller vs. larger follicles in HighVAR sows only.

\begin{tabular}{|c|c|c|c|c|c|c|}
\hline \multirow[b]{2}{*}{ Gene symbol } & \multirow[b]{2}{*}{ Gene description } & \multirow[b]{2}{*}{ Accession $\mathrm{nr}$} & \multicolumn{2}{|c|}{ HighVAR } & \multicolumn{2}{|c|}{ LowVAR } \\
\hline & & & FC & FDR & FC & FDR \\
\hline MYBL2 & MYB proto-oncogene like 2 & NM_001244469 & 3.18 & 0.01 & 2.15 & 0.05 \\
\hline FABP3 & Fatty acid binding protein 3 & XM_013999095 & 2.90 & 0.01 & 2.23 & 0.07 \\
\hline LDHA & Lactate dehydrogenase $\mathrm{A}$ & NM_001172363 & 2.58 & 0.07 & 2.50 & 0.10 \\
\hline H2AFZ & $\mathrm{H} 2 \mathrm{~A}$ histone family member $\mathrm{Z}$ & NM_001123122 & 2.57 & 0.04 & 2.29 & 0.10 \\
\hline ENSSSCT00000006872 & $\begin{array}{l}\text { Minichormosome maintenance } \\
\text { complex component } 4\end{array}$ & - & 2.39 & 0.01 & 1.92 & 0.08 \\
\hline TUBA1B & Tubulin alpha $1 b$ & NM_001044544 & 2.38 & 0.02 & 1.76 & 0.09 \\
\hline CDC45 & Cell division cycle 45 & XM_001929624 & 2.23 & 0.02 & 1.85 & 0.05 \\
\hline CDK1 & Cyclin dependent kinase 1 & NM_001159304 & 2.21 & 0.04 & 1.8 & 0.09 \\
\hline PPIA & Peptidylprolyl isomerase A & NM_214353 & 2.06 & 0.02 & 1.95 & 0.03 \\
\hline KPNA2 & Karyopherin subunit alpha 2 & NM_001163404 & 2.06 & 0.03 & 2.06 & 0.05 \\
\hline $\mathrm{AHCY}$ & Adenosylhomocysteinase & NM_001011727 & 2.02 & 0.02 & 1.60 & 0.10 \\
\hline RANBP1 & RAN binding protein 1 & NM_001185070 & 1.98 & 0.01 & 1.65 & 0.06 \\
\hline FEN1 & Flap structure-specific endonuclease 1 & XM_003122627 & 1.95 & 0.05 & 1.56 & 0.10 \\
\hline SEPP1 & Selenoprotein P & NM_001134823 & -1.92 & 0.03 & -1.83 & 0.06 \\
\hline SNRPD3 & $\begin{array}{l}\text { Small nuclear ribonucleoprotein D3 } \\
\text { polypeptide }\end{array}$ & NM_001243426 & 1.89 & 0.01 & 1.60 & 0.05 \\
\hline RAD51 & RAD51 recombinase & NM_001123181 & 1.89 & 0.01 & 1.89 & 0.06 \\
\hline MIF & Macrophage migration inhibitory factor & NM_001077213 & 1.88 & 0.06 & 1.70 & 0.09 \\
\hline LOC100156741 & Unknown & - & 1.86 & 0.02 & 1.66 & 0.06 \\
\hline HSP90AA1 & $\begin{array}{l}\text { Heat shock protein } 90 \text { alpha family } \\
\text { class A member } 1\end{array}$ & NM_213973 & 1.82 & 0.02 & 1.62 & 0.09 \\
\hline ALDOC & Aldose, fructose-bisphosphate $C$ & NM_001243928 & 1.82 & 0.02 & 1.79 & 0.03 \\
\hline
\end{tabular}


Supplemental Table S3.4 - Top 10 pathways differently expressed in smaller versus larger follicles of HighVAR sows only, using false discovery rate (FDR) $\leq 0.10$ genes.

\begin{tabular}{llccc} 
& Pathway $^{1}$ & Number $^{2}$ & Total $^{3}$ & FDR \\
\hline $\mathbf{1}$ & $\begin{array}{l}\text { Transcriptional regulation by the AP-2 family of transcription } \\
\text { factors }\end{array}$ & 9 & 52 & $5.87 \mathrm{E}-1$ \\
$\mathbf{2}$ & $\begin{array}{l}\text { SRP-dependent co-translational protein targeting to } \\
\text { membrane }\end{array}$ & 14 & 119 & $5.87 \mathrm{E}-1$ \\
$\mathbf{3}$ & Peptide chain elongation & 12 & 97 & $5.87 \mathrm{E}-1$ \\
$\mathbf{4}$ & TFAP2 family regulates transcription of cell cycle factors & 3 & 6 & $5.87 \mathrm{E}-1$ \\
$\mathbf{5}$ & Transcriptional activation of cell cycle inhibitor p21 & 3 & 6 & $5.87 \mathrm{E}-1$ \\
$\mathbf{6}$ & Transcriptional activation of p53 responsive genes & 3 & 6 & $5.87 \mathrm{E}-1$ \\
$\mathbf{7}$ & Eukaryotic Translation Elongation & 12 & 102 & $5.87 \mathrm{E}-1$ \\
$\mathbf{8}$ & Formation of a pool of free 40S subunits & 12 & 106 & $5.87 \mathrm{E}-1$ \\
$\mathbf{9}$ & Smooth muscle contraction & 8 & 55 & $5.87 \mathrm{E}-1$ \\
$\mathbf{1 0}$ & ATF6 activates chaperone genes & 4 & 15 & $5.87 \mathrm{E}-1$ \\
\hline
\end{tabular}

${ }^{1}$ Pathway analysis was performed using Reactome, ${ }^{2}$ Number of significantly different genes in the pathway,

${ }^{3}$ Total number of genes in the pathway. 
Supplemental Table S3.5 - The 20 genes with a false discovery rate (FDR) $\leq 0.1$ having the greatest fold-change (FC) between smaller versus larger follicles of LowVAR sows only.

\begin{tabular}{|c|c|c|c|c|c|c|}
\hline \multirow[b]{2}{*}{ Gene symbol } & \multirow[b]{2}{*}{ Gene description } & \multirow[b]{2}{*}{ Accession $\mathrm{nr}$} & \multicolumn{2}{|c|}{ Log2 expression } & \multirow[b]{2}{*}{ FC } & \multirow[b]{2}{*}{ FDR } \\
\hline & & & $\begin{array}{c}\text { Small } \\
\text { follicles }\end{array}$ & $\begin{array}{c}\text { Large } \\
\text { follicles }\end{array}$ & & \\
\hline ADAMTS1 & $\begin{array}{l}\text { ADAM metallopeptidase with } \\
\text { thrombospondin type } 1 \text { motif } 1\end{array}$ & NM_001144843 & 7.01 & 7.76 & -1.68 & 0.06 \\
\hline PPP1CC & $\begin{array}{l}\text { Protein phosphatase } 1 \text { catalytic subunit } \\
\text { gamma }\end{array}$ & NM_001044560 & 8.24 & 7.52 & 1.64 & 0.02 \\
\hline PDHB & $\begin{array}{l}\text { Pyruvate dehydrogenase E1 beta } \\
\text { subunit }\end{array}$ & NM_001244398 & 8.13 & 7.52 & 1.52 & 0.10 \\
\hline KHNYN & $\mathrm{KH}$ and NYN domain containing & XM_001927139 & 8.12 & 8.71 & -1.50 & 0.09 \\
\hline ATP5J2 & ATP synthase membrane subunit $f$ & NM_001097464 & 11.00 & 10.47 & 1.45 & 0.08 \\
\hline APEX1 & $\begin{array}{l}\text { Apurinic/apyrimidinic } \\
\text { endodeoxyribonuclease } 1\end{array}$ & NM_001139471 & 7.24 & 6.72 & 1.43 & 0.05 \\
\hline DPF3 & Double PHD fingers 3 & XM_021099467 & 6.56 & 6.04 & 1.43 & 0.10 \\
\hline TMEM47 & Transmembrane protein 47 & XM_001925136 & 7.81 & 8.31 & -1.42 & 0.10 \\
\hline SFRS18 & $\begin{array}{l}\text { PNN interacting serine and arginine } \\
\text { rich protein }\end{array}$ & NM_001113439 & 9.81 & 10.27 & -1.37 & 0.07 \\
\hline PPP4R3A & $\begin{array}{l}\text { Protein phosphatase } 4 \text { regulatory } \\
\text { subunit } 3 A\end{array}$ & XM_005666398 & 12.10 & 12.54 & -1.35 & 0.08 \\
\hline ARID4A & AT-rich interaction domain $4 \mathrm{~A}$ & NM_001244179 & 6.28 & 6.70 & -1.33 & 0.05 \\
\hline JMJD6 & $\begin{array}{l}\text { Jumonji Domain Containing 6, Arginine } \\
\text { Demethylase and Lysine Hydroxylase }\end{array}$ & XM_021066561 & 8.40 & 7.90 & -1.40 & 0.10 \\
\hline PSMG1 & Proteasome assembly chaperone 1 & NM_001244457 & 7.63 & 7.14 & -1.40 & 0.10 \\
\hline PSMC1 & Proteasome $26 \mathrm{~S}$ subunit, ATPase 1 & XM_021098047 & 9.89 & 9.44 & -1.37 & 0.10 \\
\hline HPRT1 & $\begin{array}{l}\text { Hypoxanthine } \\
\text { phosphoribosyltransferase } 1\end{array}$ & NM_001032376 & 6.73 & 6.35 & -1.31 & 0.10 \\
\hline CCS & $\begin{array}{l}\text { Copper chaperone for superoxide } \\
\text { dismutase }\end{array}$ & NM_001001866 & 8.30 & 7.92 & -1.30 & 0.10 \\
\hline SSPN & Sarcospan & XM_003126415 & 8.15 & 8.52 & 1.30 & 0.06 \\
\hline ТВCB & Tubulin folding cofactor B & NM_001205403 & 7.53 & 7.19 & -1.27 & 0.10 \\
\hline LOC100627373 & Unknown & - & 6.65 & 6.31 & -1.27 & 0.10 \\
\hline NACC1 & Nucleus accumbens associated 1 & XM_003123340 & 6.99 & 6.68 & -1.24 & 0.10 \\
\hline
\end{tabular}




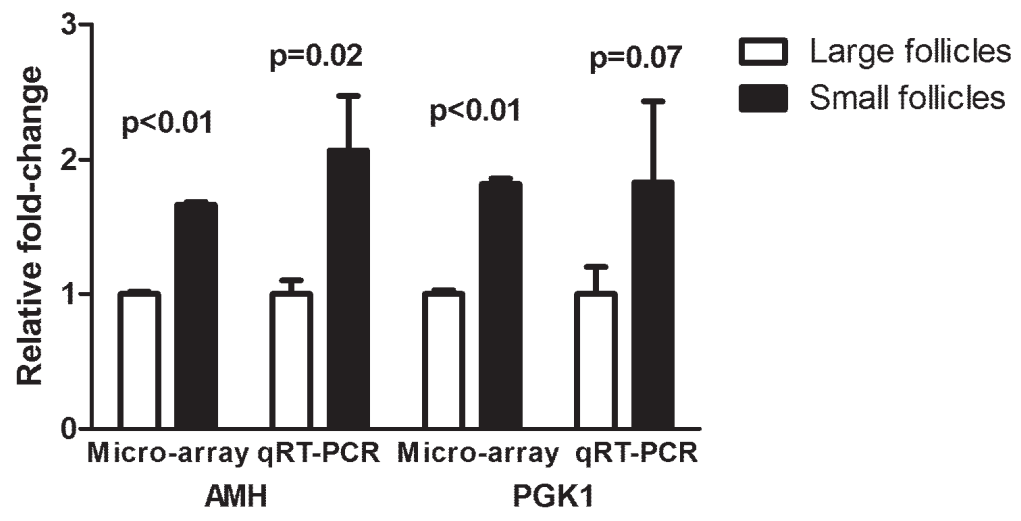

Supplemental Figure S3.1 - Relative fold-change of $A M H$ and $P G K 1$ gene expression of small versus large follicles of HighVAR sows, measured with microarrays and qRT-PCR. For AMH, 2 probes were present on the microarrays. The probe with the highest fold-change is depicted. Data are presented as mean \pm SEM $(\mathrm{N}=11 / 12)$. 



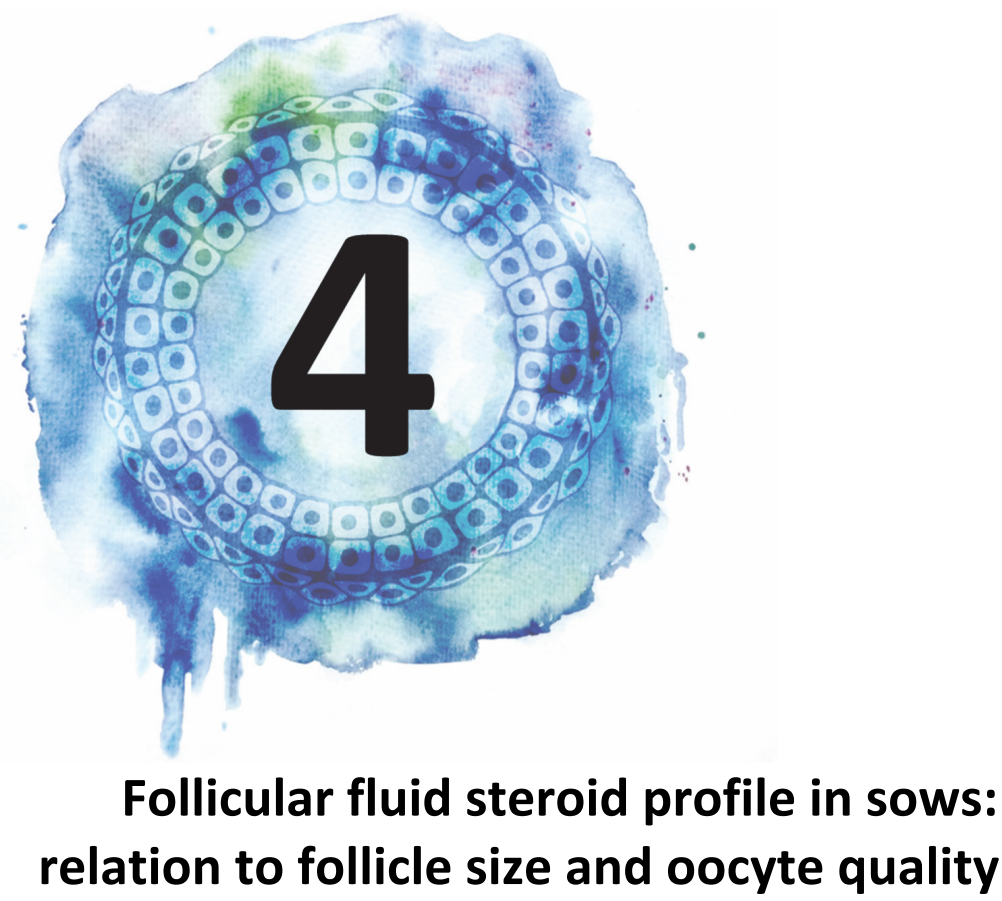

Natasja G. J. Costermans ${ }^{1}$, Nicoline M. Soede ${ }^{2}$, Frederike van Tricht $^{3}$, Marco Blokland ${ }^{3}$, Bas Kemp ${ }^{2}$, Jaap Keijer ${ }^{1} \&$ Katja J. Teerds ${ }^{1}$

${ }^{1}$ Human and Animal Physiology, Wageningen University and Research, The Netherlands

${ }^{2}$ Adaptation Physiology Group, Wageningen University and Research, The Netherlands ${ }^{3}$ Wageningen Food Safety Research, Wagenigen University and Research, The Netherlands 



\begin{abstract}
Identification of reliable characteristics of follicle quality and developmental competence has been pursued in numerous studies, but with inconsistent outcomes. Here, we aimed to identify these characteristics by analysis of the follicular fluid (FF) steroid profile in relation to cumulus-oocyte complex (COC) morphology and follicle size, followed by molecular substantiation. Multiparous sows at weaning were used to facilitate analysis at the start of the follicular phase of the oestrus cycle. Sows with a higher average follicle size ( $\geq 5 \mathrm{~mm}$ vs. $<5 \mathrm{~mm}$ ) had higher follicular fluid $\beta$-estradiol levels but did not differ in other measured steroids. Sows with high compared to low percentage healthy COCs ( $<70 \%$ vs. $\geq 70 \%$ healthy), had higher $\beta$ estradiol, 19-norandrostenedione, progesterone and $\alpha$-testosterone levels while cortisol levels were lower. Transcriptome analysis of granulosa cells of healthy follicles of sows with a high percentage healthy COCs showed higher expression of genes involved in ovarian steroidogenesis (e.g. CYP19A2 and 3, POR, SPP1) and growth (IGF1), and differential expression of genes involved in granulosa cell apoptosis (e.g. GADD45A, INHBB). Selected gene expression of aromatase (CYP19A1, 2 and 3) was confirmed at the protein level. In addition, sows with a high percentage healthy COCs lost less weight during lactation and had higher serum IGF1 levels at weaning, which may have affected COC health. To the best of our knowledge, this study is also the first to report the relation between FF steroid profile and cOC health.
\end{abstract}

Keywords: steroid hormones, granulosa cells, metabolism, insulin-like growth factor, porcine, follicle 


\section{Introduction}

During ovarian follicular development, most follicles degenerate via follicular atresia, while only a few follicles reach the pre-ovulatory state from which an oocyte ovulates to produce viable offspring (reviewed by McGee and Hsueh, 2000). Hence, from a reproductive point of view, selection of the best quality follicles is of utmost importance. It is therefore not surprising that a vast number of studies have been dedicated to identifying reliable predictors of follicle and oocyte quality, which could be useful for the selection of oocytes with high developmental competence in in vitro fertilization (IVF) procedures (as reviewed by Broekmans et al., 2006 and Rienzi et al., 2010).

Some studies have investigated relations between morphological characteristics of follicles or cumulus-oocyte complexes (COCs) and oocyte developmental competence, with very variable results. A number of these studies reported the presence of relations between antral follicle size and oocyte developmental competence, where oocytes from larger antral follicles show an increased blastocyst formation rate and implantation rate (e.g. Algriany et al., 2004; Lequarre et al., 2005). Other studies did not find such relations (e.g. Pavlok et al., 1992; Seneda et al., 2001). Distinct morphological classifications have also been used to assess oocyte competence, such as the number of cumulus cell layers (Yuan et al., 2005), darkness of the ooplasm (Nagano et al., 2006) and number of oocyte anomalies (Mikkelsen and Lindenberg, 2001), again with variable outcomes (reviewed in Wang and Sun, 2006). Follicular fluid (FF) steroid profiling could provide a new tool to identify reliable markers for follicle quality and developmental competence, as FF steroid composition determines the microenvironment in which the oocyte develops. For example, estradiol and progesterone can improve oocyte developmental competence during in vitro maturation (IVM) in porcine and bovine, when administered using optimal timing and dosing (e.g. Yuan et al., 2016a; Matsuo et al., 2017). Also, in porcine, higher cleavage and blastocyst formation rates were found when oocytes were matured in FF with higher testosterone and androstenedione levels during IVM (Grupen et al., 2003). FF steroid levels can therefore influence oocyte developmental competence.

The aim of this study was to identify potential markers for follicle quality, by studying relations between FF steroid profile, $\mathrm{COC}$ morphology and follicle size. As FF composition is also dependent on the steroidogenic activity of granulosa cells (Fortune, 1994), we additionally analysed granulosa cell gene expression using whole-genome transcriptome analysis to explain underlying mechanisms of sow differences in COC morphology and FF steroid profiles. Studies on follicle and oocyte developmental capacity have mainly used widely available prepubertal porcine or bovine ovaries from the slaughterhouse, where the stage of the oestrus cycle is not well controlled or absent. Therefore, we used sows at the moment of weaning, as sows have a well-defined start of the follicular phase at the end of lactation (reviewed by Soede et al., 2011). In many animal species, but especially in sows, the metabolic state highly influences follicular development and FF content (e.g. Quesnel et al., 1998; Yang et al., 2000). 
Therefore, we additionally analysed the sows metabolic state and its relations with follicular development and FF content.

\section{Materials and Methods}

The experiment was approved by the Animal Care and Use Committee of Wageningen University (DEC2016036) and performed according to national and EU guidelines.

\section{Animals}

A total of 29 multiparous Dutch Landrace sows (parity 3 to 5; Topigs Norsvin, Vught, the Netherlands) with an average parity of $3.8 \pm 0.2$ were used. The sows were weighed approximately 1 week before parturition and immediately after weaning. Sow weight after parturition was estimated to calculate body weight loss during lactation, as described in Costermans et al. (2019a).

\section{Blood and ovary collection}

After a lactation of $26.1 \pm 0.2$ days, sows were killed by stunning and exsanguination within 2 hours after weaning. Blood was collected in $9 \mathrm{ml}$ ice-cold EDTA activator tubes (Greiner BioOne, Monroe, NC, USA) and centrifuged at $3000 \times g$ for $10 \mathrm{~min}$ at $4{ }^{\circ} \mathrm{C}$ to collect plasma. The samples were stored at $-20^{\circ} \mathrm{C}$ until further analysis. Left ovaries were immediately placed in an insulated container and after arrival in the lab placed in PBS pH 7.4 in a water bath at $37^{\circ} \mathrm{C}$ until follicle aspiration. Right ovaries were immediately snap-frozen in liquid $\mathrm{N}_{2}$ and stored in $-80^{\circ} \mathrm{C}$ until follicle size, follicle health and gene expression analysis were performed.

\section{Measurements}

The experimental procedure used to assess ovarian measurements is summarized in Fig. 4.1. Left ovary:

COC morphology + amount of follicular fluid

The 15 largest follicles of the left ovary were used for follicle aspiration within 5 hours from slaughter. The contents of the 15 follicles were pooled and collected in a tube and allowed to settle for $5 \mathrm{~min}$. The supernatant was removed and centrifuged at $1900 \times \mathrm{g}$ at $4{ }^{\circ} \mathrm{C}$ for $30 \mathrm{~min}$ to separate cells from the follicular fluid. The total volume of follicular fluid was assessed using reverse pipetting and subsequently stored at $-20{ }^{\circ} \mathrm{C}$ until further analysis. The recovered cumulus-oocyte complexes (COCs) were morphologically classified under a dissection microscope as healthy (intact cumulus and normal-shaped oocyte) or unhealthy (degraded cumulus or degenerated oocyte) similar to Alvarez et al. (2009). For each sow, the percentage healthy COCs was calculated.

\section{Steroid profiling}

The follicular fluid of the 15 largest follicles of the left ovary was pooled to obtain a sufficient sample volume needed for endogenous steroid hormone profiling. A modified UHPLC-MS/MS method as described in Blokland et al. (2017) was used to detect endogenous aglycons in FF. 
In short: $900 \mu \mathrm{l}$ water was added to $100 \mu \mathrm{l}$ follicular fluid and followed by solid phase extraction using an Oasis HLB 96-well SPE plate (Waters, Milford, CA, USA), derivatization of aglycons was performed with picolinic acid. Chromatographic separation of the aglycons was achieved on a Waters BEH $\mathrm{C}_{18}$ column (Waters) followed by analysis on a Xevo TQS mass spectrometer (Waters) in positive ESI mode.

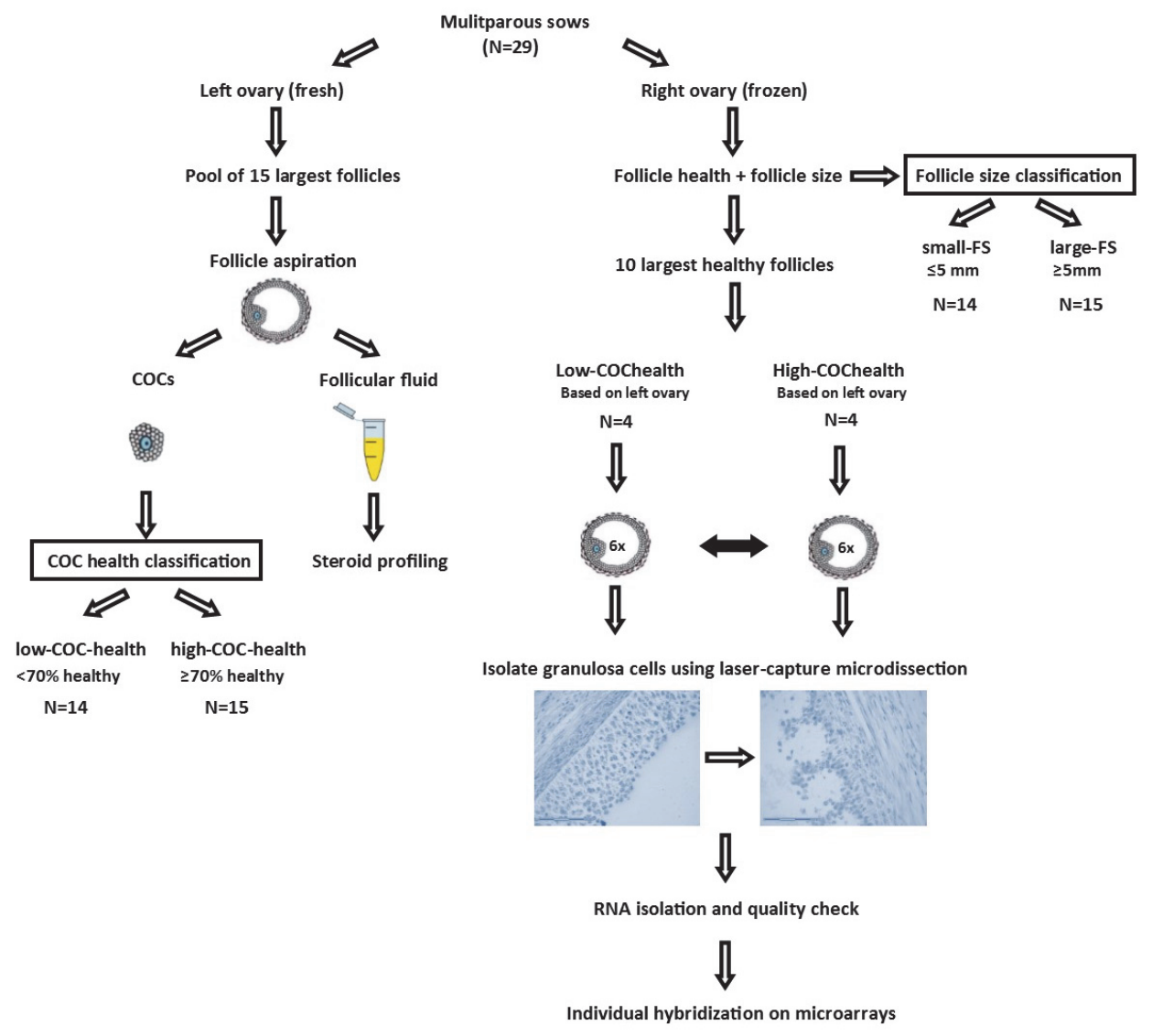

Figure 4.1 - Of a total of 29 multiparous sows, left ovaries were used for follicle aspiration of the 15 largest follicles. Follicular fluid was used for steroid profiling using a modified UHPLC-MS/MS method and cumulus-oocyte complexes (COCs) were morphologically classified as healthy or unhealthy to determine the level of $\mathrm{COC}$ health for each sow. Sows with $<70 \%$ healthy COCs were classified as lowCOC-health, sows with $\geq 70 \%$ healthy COCs as high-COC-health. Right ovaries were used to determine follicle size and health status of all visible follicles of the right ovary, by using a cleaved-Caspase 3 staining. Average follicle size of the 15 largest follicles was used to determine the follicle size class (small-FS $<5 \mathrm{~mm}$ vs. large-FS $\geq 5 \mathrm{~mm}$ ). A subset of 8 out of 29 sows were used for whole-genome transcriptome analysis of granulosa cells, of which 4 were classified as low-COC-health $(54 \pm 12 \%$ healthy COCs) and 4 were classified as high-COC-health ( $93 \pm 15 \%$ healthy COCs), as based on the left ovary. For each sow, granulosa cells of 6 healthy follicles of the pool of 10 largest healthy follicles were isolated using laser capture microdissection, after which samples were individually hybridized onto whole-genome microarrays for whole- genome transcriptome analysis. 


\section{Right ovary:}

Follicle size + follicle health

Right frozen ovaries were held against a ruler and photographed from different sides to measure the size of the 15 largest follicles using ImageJ (version 1.51f, National Institutes of Health). These 15 largest follicles were assumed to represent approximately half of the ovulatory follicle pool, as ovulation rates in modern sows are around 25-30 (da Silva et al., 2016). Subsequently, follicle health was assessed using an immunohistochemical staining against the apoptotic marker cleaved-Caspase 3 (cCASP), as described in Costermans et al. (2019a). Follicles were classified as atretic when they had $>5 \%$ positively stained granulosa cells.

\section{Gene expression granulosa cells}

A subset of 8 out of 29 sows were used for whole-genome transcriptome analysis of granulosa cells, of which 4 were classified as low-COC-health $(54 \pm 12 \%$ healthy COCs of the largest 15 follicles) and 4 were classified as high-COC-health ( $93 \pm 15 \%$ healthy COCs), based on left ovary classification. The right ovary was used for transcriptome analysis as it is essential for this analysis to obtain high quality intact RNA, for which the ovaries needed to be immediately frozen. The subset of sows which was used for transcriptome analysis had a similar average follicle size $(5.1 \pm 0.1 \mathrm{~mm}$ vs. $5.0 \pm 0.1 \mathrm{~mm}$ in low-COC-health and high-COC-health, respectively). In short, for each of the 8 sows, granulosa cells of 6 individual healthy follicles of the pool of 10 largest healthy follicles, were isolated using laser-capture microdissection and individually hybridized onto 4x44K Agilent whole-porcine genome arrays (G2519F-026440, Agilent Technologies Inc., Santa Clara, CA, USA) as described in Costermans et al. (2019b). Microarray data have been deposited in NCBI Gene Expression Omnibus (GEO) under accession number GSE125189. Fold-change is expressed as the ratio of the normalised expression of sows with low-COC-health ( $<70 \%$ healthy COCs) over sows with high-COC-health ( $>70 \%$ healthy COCs).

\section{Aromatase immunostaining}

The right ovary of the sows was used for quantification of aromatase protein expression. A subset of 12 out of 29 sows were selected for analysis, 6 sows with Low-COC-health (37 $\pm 19 \%)$ and 6 sows with High-COC-health $(98 \pm 5 \%)$, based on left ovary classification. For each of the 12 sows, both the largest and smallest follicle of the 10 largest healthy follicles was analysed. Cryosections were mounted on Superfrost plus glass slides (Menzel- Gläser, Braunschweig, Germany), air-dried for 30 minutes and fixed in 4\% PAF for 10 minutes. Slides were subsequently washed in $\mathrm{H}_{2} \mathrm{O}$, microwaved in sub-boiling $0.1 \mathrm{M}$ sodium citrate buffer $(\mathrm{pH} 6)$ for $10 \mathrm{~min}$ for epitope antigen retrieval, cooled down to room temperature and next rinsed with PBS pH 7.4. Aldehyde residues were blocked with $0.3 \%$ glycine in PBS for $10 \mathrm{~min}$. After rinsing with PBS, sections were pre-incubated with $5 \%$ (wt/v) normal goat serum in PBS for 60 min at room temperature. Subsequently, the sections were incubated overnight at $4^{\circ} \mathrm{C}$ in a humid chamber with anti-aromatase primary antibody (ab18995, lotnr:GR3231482-1, Abcam, 
Cambridge, UK) diluted 1:50 in PBS-BSA-c (Aurion, Wageningen, The Netherlands). Next, sections were rinsed with PBS and incubated in the dark with a secondary Alexa fluor 488 labelled goat-anti-rabbit antibody (A-11008, ThermoFisher Scientific, Waltham, MA, USA) diluted 1:200 (v/v) in PBS-BSA-c for 1 hour at room temperature. Sections were counterstained with DAPI ( $1 \mu \mathrm{g} / \mathrm{ml}$; Sigma-Aldrich, Saint Louis, MO, USA) for 10 minutes. Sections were imaged at 10 times magnification using a fluorescence microscope (Leica DM6B), a digital camera (DFC365 FX) and imaging software (LasX; all Leica Microsystems, Amsterdam, The Netherlands). Mean staining intensity was determined using ImageJ for the complete intact granulosa cell layer. Those parts of the granulosa layer that were visually not intact, were excluded from the analysis.

\section{IGF1 measurement}

Plasma insulin-like growth factor 1 (IGF1) content was measured by an immunoradiometric assay according to the manufacturer's protocol (A15729, Beckman Coulter, Woerden, The Netherlands) supplemented with an additional acid-ethanol extraction $(87.5 \% \mathrm{v} / \mathrm{v}$ EtOH and $2.9 \mathrm{v} / \mathrm{v} 12 \mathrm{~N} \mathrm{HCl}$ ). Samples were measured in duplicate and intra-assay coefficient of variation was $\leq 6 \%$ for all samples.

\section{Statistical analyses}

Distributions of the means and residuals were examined to verify model assumptions of normality and homogeneity of variance. Concentrations of $\beta$-Estradiol, progesterone, DHEA, $\alpha$-testosterone, $\beta$-testosterone, 11-deoxycorticosterone, 11-deoxycortisol, cortisone, cortisol, $\mathrm{DHT}, 17 \alpha-\mathrm{OH}$-progesterone and corticosterone were log transformed to obtain normality. The presence of outliers was tested by calculating the studentized residuals using the REG procedure (SAS 9.4, Cary, NC, USA); identified outliers were removed from further analyses. One outlier was removed for $\beta$-estradiol, progesterone, dehydroepiandrostenedion (DHEA), $\alpha$-testosterone, 11-deoxycortisol, 4-androsten-3,17-dione and 17 $\alpha$-OH-progesterone. Differences between follicle size (FS) classes (average follicle size of 15 largest follicles: small$\mathrm{FS}<5 \mathrm{~mm}(\mathrm{~N}=14)$ and large- $\mathrm{FS} \geq 5 \mathrm{~mm}(\mathrm{~N}=15))$ and $\mathrm{COC}$ health classes (percentage healthy COCs of the 15 largest follicles: low-COC-health<70\% ( $N=14)$ and high-COC-health $\geq 70 \%(\mathrm{~N}=15)$ ) were analysed in the same model using the procedure GLM in models that also contained the factor PAR (PAR3 (parity 3, N=14) and PAR4+5 (parity 4 and 5, N=15)) and the interactions with PAR. The interactions were excluded from the models when not significant. All values are presented as LS means \pm SE unless otherwise stated.

For microarray analysis, student's t tests were used with false discovery rate (FDR) adjustment for multiple testing correction according to Benjamini-Hochberg. Fold-change (FC) is defined as the normalised expression of high-COC-health over low-COC-health. Analysis of networks of all genes with a FDR-adjusted $p \leq 0.2$ and absolute $F C \geq 1.2$ was performed using Reactome (http://www.reactome.org), where non-assigned probes were excluded from further analysis. Analysis of differences in aromatase staining intensity was performed using a 2-way ANOVA 
(Graphpad Prism version 5.04, GraphPad Software, La Jolla, CA, USA) using COC health class and follicle size class as factors.

\section{Results}

High-COC-health sows had lost a lower percentage of their body weight during lactation and had higher IGF1 plasma levels at weaning as compared to low-COC-health sows but did not differ in follicle size or volume (see Table 4.1). In follicles of high-COC-health sows, follicular fluid $\beta$-estradiol, progesterone, 19-norandrostenedione and $\alpha$-testosterone levels were higher as compared to low-COC-health sows, while cortisol levels were lower (Fig. 4.2). Levels of other steroid levels did not differ (Supplemental Table S4.1).

Table 4.1 - Effects of follicle size (av. follicle size of 15 largest follicles: Small-FS $<5 \mathrm{~mm}, \mathrm{~N}=14$ and Large-FS $>5 \mathrm{~mm}, \mathrm{~N}=15$ ) and COC health class (percentage healthy COCs: low-COC-health $<70 \%, \mathrm{~N}=14$ and high-COC-health $>70 \%, \mathrm{~N}=15$ ) on lactation characteristics, body condition, metabolic parameters and follicular parameters.

\begin{tabular}{lcccccc} 
& \multicolumn{2}{c}{ Follicle size } & \multicolumn{2}{c}{ COC health } & \multicolumn{2}{c}{ P-values } \\
\cline { 2 - 7 } & Small & Large & Low & High & FS & COC \\
\hline Lactation & & & & & & \\
$\quad$ Number of piglets weaned & $12.6 \pm 0.3$ & $12.7 \pm 0.2$ & $12.4 \pm 0.3$ & $12.9 \pm 0.2$ & 0.99 & 0.12 \\
$\quad$ Litter growth (kg) & $71 \pm 3$ & $73 \pm 2$ & $72 \pm 3$ & $72 \pm 2$ & 0.47 & 0.99 \\
Body condition & & & & & & \\
$\quad$ Weight parturition (kg) & $254 \pm 6$ & $243 \pm 5$ & $253 \pm 6$ & $242 \pm 5$ & 0.12 & 0.14 \\
$\quad$ Weight weaning (kg) & $225 \pm 4$ & $220 \pm 4$ & $222 \pm 4$ & $224 \pm 4$ & 0.53 & 0.82 \\
Weight loss lactation (\%) & $11 \pm 2$ & $10 \pm 2$ & $12 \pm 2$ & $7 \pm 2$ & 0.92 & 0.03 \\
IGF-1 (ng/ml) & $150 \pm 17$ & $138 \pm 15$ & $120 \pm 16$ & $167 \pm 15$ & 0.62 & 0.05 \\
Follicular parameters & & & & & & \\
Av. follicle size (mm) & $4.4 \pm 0.1$ & $5.6 \pm 0.1$ & $5.0 \pm 0.1$ & $5.1 \pm 0.1$ & $<0.001$ & 0.84 \\
Amount of follicular fluid $(\mu \mathrm{l})$ & $334 \pm 40$ & $405 \pm 38$ & $363 \pm 40$ & $376 \pm 38$ & 0.22 & 0.82 \\
Healthy COCs (\%) & $67 \pm 4$ & $70 \pm 4$ & $49 \pm 4$ & $89 \pm 4$ & 0.67 & $<0.001$ \\
\hline
\end{tabular}

All values are presented as $\mathrm{LS}$ means \pm SE. COC=cumulus-oocyte complex. Follicle size was not related to percentage healthy COCs. ${ }^{1}$ LS means estimates for the interaction FS*PAR $(p<0.01)$ : Small-FS*PAR3: $228 \pm 8$, Small-FS*PAR4+5: 259 \pm 6 , Large-FS*PAR3: 252 \pm 6 , LargeFS*PAR4+5: 239 \pm 6

In large-FS sows, average follicle size of the 15 largest follicles was larger as compared to smallFS sows $(p<0.001)$. No significant effects of follicle size were found in any of the lactational or metabolic measurements (see Table 4.1). Large-FS sows had higher follicular fluid $\beta$-estradiol levels ( $5.63 \pm 0.60$ vs. $3.51 \pm 0.64 \mathrm{ng} / \mathrm{ml}, \mathrm{p}=0.03$ ) compared to small-FS sows but did not differ in levels of any of the other measured steroids (Fig. 4.3). 

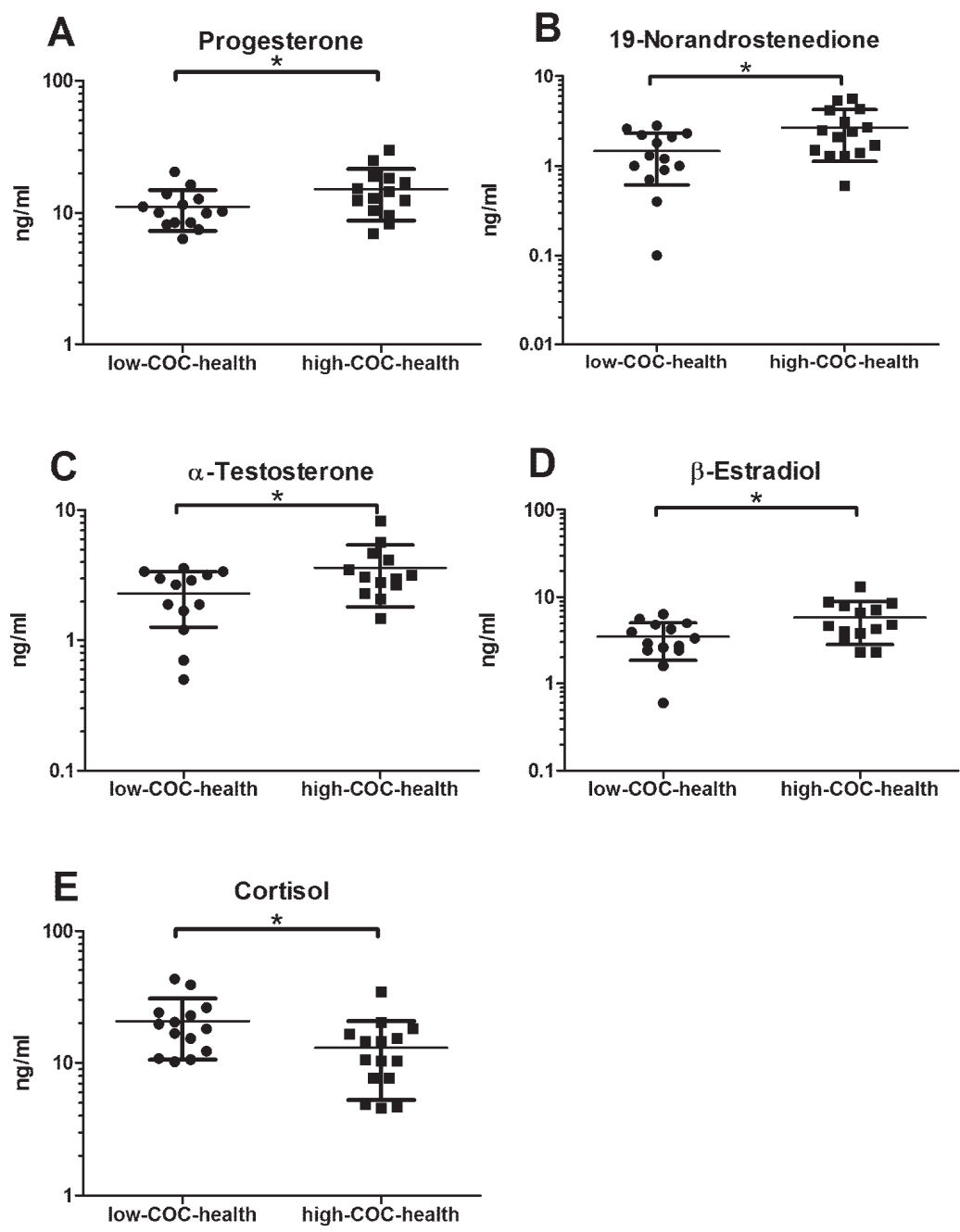

Figure 4.2 - Follicular fluid Progesterone (A) 19-Norandrostenedione (B) $\alpha$-Testosterone (C) $\beta$-Estradiol (D) and Cortisol levels $(E ; n g / m l)$ of the 15 largest follicles of the left ovary of sows with high-COChealth ( $\geq 70 \%$ healthy COCs, $N=14$ ) vs. sows with low-COC-health $(<70 \%$ healthy COCs, $N=15)$. ${ }^{*}$-value $<0.05$.

Subsequently, transcriptome analysis of whole-genome granulosa cell gene expression of healthy antral follicles of the right ovary was performed in a subset of 8 sows of which 4 were classified as high-COC-health and 4 were classified as low-COC-health. The analysis showed a total of 235 unique transcripts, which were differentially expressed. Subsequent pathway analysis revealed 14 regulated pathways (FDR $\leq 0.2$ ), which include cholesterol biosynthesis (7/72 genes, FDR 0.06), regulation of cholesterol biosynthesis via SREBPs (sterol-regulatory element binding proteins; 8/86 genes, FDR 0.06 ) and steroid metabolism (15/321 genes, FDR 0.11 ) (see Table 4.2 for details). 


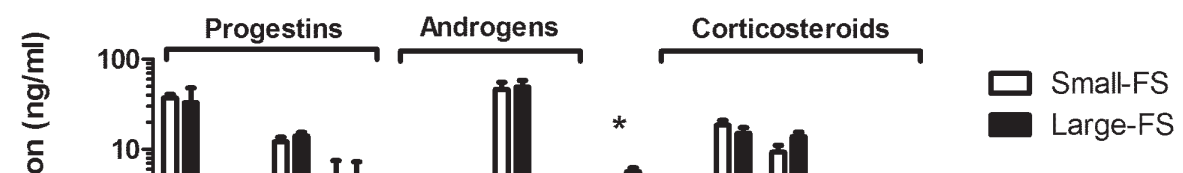

Figure 4.3 - Follicular fluid steroid levels $(\mathrm{ng} / \mathrm{ml})$ of sows with a small average follicle size (small-FS: average follicle size of the 15 largest follicles of the right ovary $\leq 5 \mathrm{~mm}, \mathrm{~N}=14$ ) vs. sows with a large follicle size (large-FS: average follicle size $\geq 5 \mathrm{~mm}, \mathrm{~N}=15$ ). ${ }^{*} \mathrm{P}$-value $<0.05$

Individual transcript analysis showed an increased expression of genes involved in ovarian steroidogenesis in sows with high-COC-health e.g. CYP19A2 and CYP19A3 (FC = 1.46 and 1.43, FDR $=0.09$ for both), secreted phosphoprotein 1 (SPP1; FC $=1.30$ and FDR $=0.14$ ) and P450 oxidoreductase $(P O R ; \mathrm{FC}=1.22, \mathrm{FDR}=0.12)$ and a decreased expression of adrenomedullin $(A D M ; F C=-1.60$ and $F D R=0.18)$ (see Table 4.3 for details). Next to genes involved in steroidogenesis, the top 10 regulated genes with a higher expression in high-COC-health sows included e.g. inhibin subunit beta B (INHBB; FC $=1.64$ and FDR $=0.05)$, glutathione Stransferase alpha 2 (GSTA2; FC $=1.42$ and FDR $=0.11)$ and metallothionein $1 \mathrm{~A}(M T 1 A ; F C=$ 1.52 and $F D R=0.07$ ) while the top 10 genes with a lower expression in high-COC-health sows includes e.g. growth arrest and DNA damage inducible alpha (GADD45A; FC $=-1.63$ and FDR = 0.18 ) (for details see Supplemental Table S4.2). In addition, granulosa cells of antral follicles in high-COC-health sows had higher IGF1 expression (FC $=1.41$ and FDR $=<0.01$ ). Pigs express multiple homologous (>99\%) isoforms of aromatase, CYP19A1, 2 and 3, which are mainly expressed in ovary, placenta and embryonic tissues, respectively (Graddy et al., 2000). As CYP19A2 and CYP19A3 were regulated, we additionally analysed aromatase (CYP19A1-3) protein expression. Sows with high-COC-health had higher aromatase protein expression as compared to sows with low-COC-health $(p<0.01)$. Aromatase protein expression did not differ between the large and small healthy antral follicles ( $p=0.27$ ) (Fig. 4.4, Supplemental Fig. S4.1). 
Table 4.2 - Significant pathways (FDR $\leq 0.2$ ) of the 235 unique transcripts which were significantly different (absolute $F C \geq 1.2, F D R \leq 0.2)$ between sows with high-COC-health $(93 \pm 15 \%$ healthy, $N=4)$ vs. sows with low-COC-health ( $54 \pm 12 \%$ healthy, $\mathrm{N}=4)$ as analysed by Reactome.

\begin{tabular}{|c|c|c|c|c|}
\hline & Pathway & Genes & Total & FDR \\
\hline 1 & Activation of gene expression by SREBF (SREBP) & 8 & 70 & 0.06 \\
\hline 2 & Regulation of cholesterol biosynthesis by SREBP (SREBF) & 8 & 86 & 0.06 \\
\hline 3 & $\begin{array}{l}\text { Deregulated CDK5 triggers multiple neurodegenerative } \\
\text { pathways in Alzheimer's disease models }\end{array}$ & 5 & 31 & 0.06 \\
\hline 4 & Neurodegenerative Diseases & 5 & 31 & 0.06 \\
\hline 5 & $\begin{array}{l}\text { Defective CSF2RB causes pulmonary surfactant metabolism } \\
\text { dysfunction } 5 \text { (SMDP5) }\end{array}$ & 3 & 8 & 0.06 \\
\hline 6 & $\begin{array}{l}\text { Defective CSF2RA causes pulmonary surfactant metabolism } \\
\text { dysfunction } 4 \text { (SMDP4) }\end{array}$ & 3 & 8 & 0.06 \\
\hline 7 & Cholesterol biosynthesis & 7 & 72 & 0.06 \\
\hline 8 & Surfactant metabolism & 6 & 53 & 0.06 \\
\hline 9 & $\begin{array}{l}\text { Gene and protein expression by JAK-STAT signaling after } \\
\text { Interleukin-12 stimulation }\end{array}$ & 7 & 74 & 0.06 \\
\hline 10 & Interleukin-12 signaling & 7 & 85 & 0.11 \\
\hline 11 & Platelet degranulation & 9 & 137 & 0.11 \\
\hline 12 & Metabolism of steroids & 15 & 321 & 0.11 \\
\hline 13 & Response to elevated platelet cytosolic $\mathrm{Ca} 2+$ & 9 & 144 & 0.13 \\
\hline 14 & Interleukin-12 family signaling & 7 & 97 & 0.17 \\
\hline
\end{tabular}

For each pathway, the number of different genes, total number of genes and significance (FDR) are shown.

Table 4.3 - Significantly different genes (absolute FC $\geq 1.2$, FDR $\leq 0.2$ ) involved in ovarian steroidogenesis (ref: Ovarian Kaleidoscope Database) between sows with high-COC-health $(93 \pm 15 \%$ of COCs healthy $(\mathrm{N}=4))$ vs. sows with low-COC-health $(54 \pm 12 \%$ of COCs healthy $(\mathrm{N}=4))$.

\begin{tabular}{lllll} 
Gene symbol & Gene description & Accession nr & FC & FDR \\
\hline GSTA2 & Glutathione S-transferase alpha 2 & NM_213850 & 1.55 & 0.12 \\
CYP19A2 & Cytochrome P450 19A2 & NM_214430 & 1.46 & 0.09 \\
CYP19A3 & Cytochrome P450 19A3 & NM_214431 & 1.43 & 0.09 \\
SPP1 & Secreted phosphoprotein 1 & NM_214023 & 1.30 & 0.14 \\
SQLE & Squalene epoxidase & XM_005662853.3 & 1.28 & 0.08 \\
ENSSSCT00000010858 & TP53 regulated inhibitor of apoptosis 1 & - & 1.25 & 0.02 \\
POR & P450 oxidoreductase & NM_001129959 & 1.22 & 0.12 \\
EPHX1 & Epoxide hydrolase 1 & NM_214355 & 1.21 & 0.04 \\
LDLR & Low density lipoprotein receptor & NM_001206354 & 1.20 & 0.13 \\
ADM & Adrenomedullin & NM_214107 & -1.60 & 0.18 \\
KLF13 & Kruppel like factor 13 & NM_001011505 & -1.28 & 0.05 \\
STC1 & Stanniocalcin 1 & NM_001103212 & -1.25 & 0.12 \\
GHR & Growth hormone receptor & NM_214254 & -1.24 & 0.14 \\
THBD & Thrombomodulin & NM_001130732 & -1.24 & 0.14 \\
\hline For each gene, the fold-change (FC) of high-COC-health sows vs. low-COC-health sows and significance (FDR) \\
are shown.
\end{tabular}




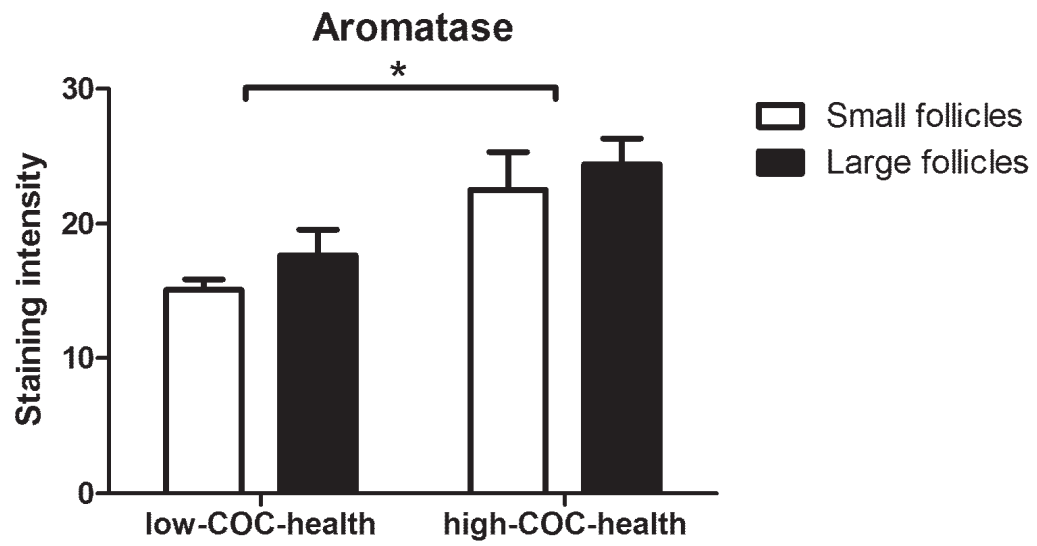

Figure 4.4 - Aromatase protein expression in granulosa cells of healthy follicles of sows with high-COChealth $(98 \pm 5 \%$ of COCs healthy $(N=6))$ vs. low-COC-health $(37 \pm 19 \%$ of COCs healthy $(N=6))$ in the smallest and largest follicle of the 10 largest healthy follicles. Data are presented as average \pm SD. *Pvalue $<0.05$.

\section{Discussion}

The aim of this study was to identify potential markers for antral follicle quality. We investigated relations between follicular fluid steroid levels, follicular development and COC morphology in multiparous sows at the onset of the follicular phase of the oestrus cycle. To our knowledge, this is the first study in which porcine follicular fluid steroid profiling is reported, let alone in follicles at a specific stage of the oestrus cycle.

Sows at the start of the follicular phase of the oestrus cycle with a large vs. small average follicle size had higher follicular fluid $\beta$-estradiol levels. In pigs, granulosa cell CYP19 expression and subsequent $\beta$-estradiol production increases from the start of the follicular phase until the pre-ovulatory stage approximately 5 days later (Grant et al., 1989), and consequently as follicles develop, follicular fluid $\beta$-estradiol levels increase with follicular diameter. In most studies, effects of follicle size on steroid levels were either not investigated in ovaries which were in the same stage of the oestrus cycle (e.g. Ainsworth et al., 1980; Liu et al., 2000; Bertoldo et al., 2011) or were only investigated at the final stages of follicular development, from pro-oestrus to late oestrus (e.g. Conley et al., 1994). The results of our study are the first to indicate that from the pool of antral follicles, the larger subset of follicles have increased $\beta$ estradiol levels already at the start of the follicular phase. Having said this, follicle size is not related to any of the other measured follicular fluid steroids. Follicular progesterone production is highest in the early luteal stage and only starts to increase in the latest stages of the follicular phase (Liu et al., 2000; Mahajan, 2008), which can explain why we did not find a relation between follicular size and follicular fluid progesterone levels. Although high levels of testosterone and androstenedione are present during the entire follicular phase (Ainsworth 
et al., 1980; Majahan, 2008), these androgens can also be quickly aromatized into $\beta$-estradiol, which might explain why we did not find a relation between follicle size and testosterone and androstenedione steroid levels.

Next, we compared the follicular fluid steroid profile of sows with high vs. low percentage of healthy COCs. COC morphology was used as a marker for oocyte quality as COCs with an intact cumulus layer and normal shaped oocytes have the highest developmental capacity in vitro (Alvarez et al., 2009). Sows with a high percentage healthy COCs had higher FF $\beta$-estradiol, progesterone, 19-norandrostenedione and $\alpha$-testosterone levels as compared to sows with a low percentage of healthy COCs at the start of the follicular phase. Similarly, studies in pigs have shown that in early atretic antral follicles, the expression of follicle-stimulating hormone receptor (FSHR), luteinizing hormone receptor (LHCGR) and CYP19 is lower (Tilly et al., 1992; Pan et al., 2012) resulting in lower FSH-induced estradiol production (Westhof et al., 1991; Guthrie and Cooper, 1996) and lower in vitro cumulus cell progesterone production (Grupen and Armstrong, 2010). It therefore seems reasonable to assume that the follicle pool of 15 largest follicles from which the COCs were isolated and where we did not discriminate between healthy and atretic follicles, contained a higher fraction of atretic follicles in sows with a low percentage healthy COCs. Besides higher $\beta$-estradiol, progesterone, 19norandrostenedione and $\alpha$-testosterone levels, we found lower cortisol levels in follicular fluid of sows with a higher percentage of healthy COCs. As cortisol is an important regulator of stress-related apoptosis of granulosa cells and oocytes via the FAS system (Yuan et al., 2016b), differences in FF cortisol levels can at least partly explain the observed differences in COC health levels.

To further elucidate underlying mechanisms of differences in $\mathrm{COC}$ health and FF fluid steroid content in sows, we performed whole-genome transcriptome analysis in granulosa cells. We used a subset eight sows to analyse mural granulosa cell gene expression in healthy follicles only. This transcriptome analysis revealed an increased expression of genes involved in pathways responsible for steroid and cholesterol biosynthesis in sows with a high percentage healthy COCs. Two of these transcripts (CYP19A2 and CYP19A3) encode for cytochrome P450 aromatase, the granulosa cell terminal enzyme in the estrogen biosynthetic pathway responsible for the conversion of androgens into estradiol (Conley et al., 1994). Immunostaining against aromatase (CYP19A1-3) confirms the increased aromatase expression in granulosa cells of sows with a high percentage of healthy COCs at the protein level. This increased granulosa cell aromatase expression may explain the higher FF $\beta$-estradiol levels in sows with high $\mathrm{COC}$ health. In addition, although not differentially regulated, our granulosa cell transcriptome analysis showed that granulosa cells also express other steroidogenic enzymes already at the start of the follicular phase; steroidogenic acute regulatory protein (STAR), regulating cholesterol transfer within the mitochondria (Clark et al., 1994), cytochrome P450 family 11 subfamily A member 1 (CYP11A1) and hydroxysteroid 17- $\beta$ 
dehydrogenase 1 (HSD17B1), involved in the conversion of cholesterol into androgens (reviewed by Payne and Hales, 2004) (data not shown). Similarly, in a study by Garmey et al. (2000), granulosa cells of cyclic gilts expressed very low amounts of CYP11A and STAR mRNA and protein from approximately day 3 or 5 , respectively, of the follicular phase. Apparently, porcine follicular development differs from that of rats and mice, as granulosa cells in the latter species only start to express CYP11A and STAR in pre-ovulatory follicles, so at a later stage of follicular development (e.g. reviewed by Ronen-Fuhrmann et al., 1998 and Payne and Hales, 2004). As CYP11A1, STAR and HSD17B1 were expressed in granulosa cells, they might contribute to follicular androgen production in sows, already at the start of the follicular phase. However, these steroidogenic enzyme transcripts were not differentially regulated in granulosa cells of sows with high vs. low percentage healthy COCs and can therefore not explain the higher progesterone, 19-norandrostenedione and $\alpha$-testosterone levels in FF of sows with high $\mathrm{COC}$ health. Possibly, these steroidogenic enzymes might be differentially regulated at the protein level or have a different enzymatic activity. Thus, although granulosa cells contribute to follicular androgen production in sows, they are at least at this stage of follicular development not responsible for differentially regulating androgen levels in FF. This confirms the existence of the classical two-cell, two-gonadotropin concept for estrogen biosynthesis in the porcine ovary, in which theca cells play the major role in androgen production and its regulation (reviewed by Magoffen, 2005).

Besides genes encoding enzymes participating in steroid metabolism, sows with a high vs. low percentage healthy COCs had a differential expression of genes, which are involved in regulation of enzymes involved in ovarian steroidogenesis. Examples of these differentially expressed genes are SPP1, POR and ADM. Sows with a high percentage healthy COCS had a higher expression of SPP1 and POR. SPP1 stimulates ovarian steroidogenesis in mouse granulosa cells at the pre-ovulatory follicle stage by increasing gene expression levels of the enzymes STAR, CYP11A1, hydroxy-delta-5-steroid dehydrogenase $3 \beta$ (HSD3B) and consequently progesterone synthesis (Kuwabara et al., 2015). POR supplies electrons to all CYP enzymes for catalytic activity and is essential for steroid biosynthesis (reviewed by Miller et al., 2011). ADM has been identified as a potential paracrine factor inhibiting FSH-induced estradiol secretion in rats (Li et al., 2008), and was lower expressed in the sows of our study with a higher percentage healthy COCs. Therefore, higher SPP1 and POR and lower ADM might be involved in increasing ovarian steroid biosynthesis at the start of the follicular phase in sows with a high percentage healthy COCs.

Next to genes involved in ovarian steroidogenesis, we also found differential expression of genes regulating ovarian follicular atresia, e.g. INHBB and GADD45A. GADD45A expression was lower in sows with a high percentage healthy COCs. GADD45A is involved in the DNA damage response and has a higher expression in porcine atretic follicles (Terenina et al., 2016). GADD45A might provide a valuable marker for early atretic follicles, as it was already 
expressed in the antral follicles of our study which have been classified as healthy, as they did not (yet) express cleaved-Caspase 3. Granulosa cell INHBB expression was higher in sows with had a high percentage healthy COCs. INHBB encodes for the B subunit of activin and inhibin complexes. Follicular atresia has been previously associated with lower levels of INHBB in porcine granulosa cells (Terenina et al., 2016; Tu et al., 2014). Other genes that had a higher expression in granulosa cells of sows with a high percentage healthy COCs, encode for proteins that are protective against oxidative stress e.g. GST and MT1A. Both GST and MT1A might play a role in protecting the ovarian tissues from oxidative stress (Epsey et al., 2003; Alscher et al., 2005). High levels of reactive oxygen species are produced during steroid biosynthesis which results in the increased expression of superoxide dismutase, catalase and glutathione peroxidase in steroid secreting areas of the ovary (reviewed by Hanukoglu, 2006). In our study, follicles of sows with a high percentage healthy COCs produce more steroids, as seen by the higher levels of FF steroids and expression of genes involved in steroidogenesis, which might explain the increased expression of genes involved in the oxidative stress response.

Follicle size and health status and $\mathrm{COC}$ health can be affected by environmental factors, such as the nutritional status. In the current study, ovaries were obtained within two hours after weaning. Therefore, we analysed the lactational metabolic state of the sows as well, as this might underlie differences in follicular development (reviewed by Hazeleger et al., 2005). Sows with a higher percentage healthy COCs at the day of weaning lost a lower percentage of their body weight during lactation and had higher peripheral IGF1 levels at weaning as compared to sows with a low percentage healthy COCs. In addition, granulosa cells of sows with a high percentage healthy COCs express more IGF1. Peripheral IGF1 indirectly influences follicular development by binding to insulin and IGF1 receptors in the hypothalamus and pituitary and therefore stimulates the production of the gonadotropins FSH and LH (reviewed by Wolfe et al., 2014). In addition, both peripheral and locally produced IGF1 are essential for follicular development, as IGF1 can activate IGF1 signalling in granulosa cells and synergize with FSH to stimulate follicular growth and steroidogenesis (Xia et al., 1994). This effect is mediated by activation of phosphatidylinositol-dependent kinase/AKT, as shown for bovine granulosa cells in vitro (Mani et al., 2010). In human cumulus cells, effects of FSH on cumulus cell gene expression of genes involved in cholesterol and steroid metabolism are minimal when IGF1R is blocked (Stocco et al., 2017). This provides further evidence for the essential role of IGF1 in regulation of steroid production. Next to this, both FSH and IGF1 have been identified as anti-apoptotic factors (Guthrie et al., 1998). Therefore, the relatively low IGF1 levels in sows with a low percentage of healthy COCs might be insufficient to support follicular development and steroidogenesis resulting in an increased risk of follicular atresia. Indeed, the metabolic state of the sows during lactation seems to influence follicular development and FF content. 
In conclusion, we found that a positive relation between follicle size and follicular fluid $\beta$ estradiol levels already exists at the start of the follicular phase, as sows with larger follicles had higher $\beta$-estradiol levels. Follicles of sows with a high percentage healthy COCs contained more $\beta$-estradiol, progesterone, 19-noradrenostenedione and $\alpha$-testosterone and less cortisol, which could therefore serve as potential markers to identify early atretic follicles with decreased follicular developmental competence. By comparing gene expression in healthy follicles of sows with a high vs. low percentage healthy COCs, we identified several genes involved in granulosa cell steroidogenesis (e.g. ADM and SPP1) and genes involved in regulating granulosa cell apoptosis (e.g. GADD45A and INHBB), which could serve as markers for follicle quality.

\section{Acknowledgements}

The reported research was funded by the Wageningen Institute for Animal Sciences (WIAS) and NWO for providing the NWO-WIAS Graduate Programme 2015 grant.

\section{References}

Ainsworth, L., Tsang, B., Downey, B., Marcus, G., \& Armstrong, D. (1980). Interrelationships between follicular fluid steroid levels, gonadotropic stimuli, and oocyte maturation during preovulatory development of porcine follicles. Biology of reproduction, 23(3), 621-627.

Algriany, O., Bevers, M., Schoevers, E., Colenbrander, B., \& Dieleman, S. (2004). Follicle size-dependent effects of sow follicular fluid on in vitro cumulus expansion, nuclear maturation and blastocyst formation of sow cumulus oocytes complexes. Theriogenology, 62(8), 1483-1497.

Alscher, D. M., Braun, N., Biegger, D., Stuelten, C., Gawronski, K., Mürdter, T. E., Kuhlmann, U., \& Fritz, P. (2005). Induction of metallothionein in proximal tubular cells by zinc and its potential as an endogenous antioxidant. Kidney and blood pressure research, 28(3), 127-133.

Alvarez, G. M., Dalvit, G. C., Achi, M. V., Miguez, M. S., \& Cetica, P. D. (2009). Immature oocyte quality and maturational competence of porcine cumulus-oocyte complexes subpopulations. Biocell, 33(3), 167-177.

Bertoldo, M., Holyoake, P., Evans, G., \& Grupen, C. (2011). Follicular progesterone levels decrease during the period of seasonal infertility in sows. Reproduction in domestic animals, 46(3), 489494.

Blokland, M., van Tricht, E., van Ginkel, L., \& Sterk, S. (2017). Applicability of an innovative steroidprofiling method to determine synthetic growth promoter abuse in cattle. The journal of steroid biochemistry and molecular biology, 174, 265-275.

Broekmans, F., Kwee, J., Hendriks, D., Mol, B., \& Lambalk, C. (2006). A systematic review of tests predicting ovarian reserve and IVF outcome. Human reproduction update, 12(6), 685-718.

Clark, B. J., Wells, J., King, S. R., \& Stocco, D. M. (1994). The purification, cloning, and expression of a novel luteinizing hormone-induced mitochondrial protein in MA-10 mouse Leydig tumor cells. Characterization of the steroidogenic acute regulatory protein (StAR). Journal of biological chemistry, 269(45), 28314-28322.

Conley, A., Howard, H., Slanger, W., \& Ford, J. (1994). Steroidogenesis in the preovulatory porcine follicle. Biology of reproduction, 51(4), 655-661.

Costermans, N. G. J., Teerds, K. J., Keijer, J., Knol, E., Koopmanschap, R. E., Kemp, B., \& Soede, N. M. (2019a). Follicular development of sows at weaning in relation to estimated breeding value for within-litter variation in piglet birth weight. Animal, 13(3), 554-563. 
Costermans, N. G. J., Keijer, J., Schothorst, E. M., Kemp, B., Keshtkar, S., Bunschoten, A., Soede, N. M., \& Teerds, K. J. (2019b). In ovaries with high or low variation in follicle size, granulosa cells of antral follicles exhibit distinct size-related processes. Molecular human reproduction, 25(10), 614-624.

da Silva, C. L. A., Laurenssen, B., Knol, E., Kemp, B., \& Soede, N. (2016). Relationships between ovulation rate and embryonic and placental characteristics in multiparous sows at 35 days of pregnancy. Animal, 10(7), 1192-1199.

Espey, L., Ujioka, T., Okamura, H., \& Richards, J. (2003). Metallothionein-1 messenger RNA transcription in steroid-secreting cells of the rat ovary during the periovulatory period. Biology of reproduction, 68(5), 1895-1902.

Fortune, J. (1994). Ovarian follicular growth and development in mammals. Biology of reproduction, 50(2), 225-232.

Garmey, J. C., Guthrie, H., Garrett, W. M., Stoler, M. H., \& Veldhuis, J. D. (2000). Localization and expression of low-density lipoprotein receptor, steroidogenic acute regulatory protein, cytochrome P450 side-chain cleavage and P450 17- $\alpha$-hydroxylase/C17-20 lyase in developing swine follicles: in situ molecular hybridization and immunocytochemical studies. Molecular and cellular endocrinology, 170(1-2), 57-65.

Graddy, L., Kowalski, A., Simmen, F., Davis, S., Baumgartner, W., \& Simmen, R. (2000). Multiple isoforms of porcine aromatase are encoded by three distinct genes. The journal of steroid biochemistry and molecular biology, 73(1-2), 49-57.

Grant, S. A., Hunter, M., \& Foxcroft, G. (1989). Morphological and biochemical characteristics during ovarian follicular development in the pig. Journal of reproduction and fertility, 86(1), 171-183.

Grupen, C. G., \& Armstrong, D. T. (2010). Relationship between cumulus cell apoptosis, progesterone production and porcine oocyte developmental competence: temporal effects of follicular fluid during IVM. Reproduction, fertility and development, 22(7), 1100-1109.

Grupen, C. G., Mcllfatrick, S. M., Ashman, R. J., Boquest, A. C., Armstrong, D. T., \& Nottle, M. B. (2003). Relationship between donor animal age, follicular fluid steroid content and oocyte developmental competence in the pig. Reproduction, Fertility and development, 15(2), 81-87.

Guthrie, H., \& Cooper, B. (1996). Follicular atresia, follicular fluid hormones, and circulating hormones during the midluteal phase of the oestrus cycle in pigs. Biology of reproduction, 55(3), 543-547.

Guthrie, H., Garrett, W., \& Cooper, B. (1998). Follicle-stimulating hormone and insulin-like growth factor-I attenuate apoptosis in cultured porcine granulosa cells. Biology of reproduction, 58(2), 390-396.

Hanukoglu, I. (2006). Antioxidant protective mechanisms against reactive oxygen species (ROS) generated by mitochondrial P450 systems in steroidogenic cells. Drug metabolism reviews, 38(1-2), 171-196.

Hazeleger, W., Soede, N., \& Kemp, B. (2005). The effect of feeding strategy during the pre-follicular phase on subsequent follicular development in the pig. Domestic animal endocrinology, 29(2), 362-370.

Kuwabara, Y., Katayama, A., Tomiyama, R., Piao, H., Kurihara, S., Ono, S., Mine, K., Akira, S., Orimo, H., \& Takeshita, T. (2015). Gonadotropin regulation and role of ovarian osteopontin in the periovulatory period. Journal of endocrinology, 224(1), 49-59.

Lequarre, A.-S., Vigneron, C., Ribaucour, F., Holm, P., Donnay, I., Dalbies-Tran, R., Callesen. H., \& Mermillod, P. (2005). Influence of antral follicle size on oocyte characteristics and embryo development in the bovine. Theriogenology, 63(3), 841-859.

Li, Y.-Y., Li, L., Hwang, I. S.-S., Tang, F., \& O, W.-S. (2008). Coexpression of adrenomedullin and its receptors in the reproductive system of the rat: effects on steroid secretion in rat ovary. Biology of reproduction, 79(2), 200-208.

Liu, J., Koenigsfeld, A. T., Cantley, T. C., Boyd, C. K., Kobayashi, Y., \& Lucy, M. C. (2000). Growth and the initiation of steroidogenesis in porcine follicles are associated with unique patterns of gene expression for individual componentsof the ovarian insulin-like growth factor system. Biology of reproduction, 63(3), 942-952. 
Magoffin, D. A. (2005). Ovarian theca cell. The international journal of biochemistry \& cell biology, 37(7), 1344-1349.

Mahajan, D. K. (2008). "Pig Model to Study Dynamics of Steroids During Ovarian Follicular Growth and Maturation." In Sourcebook of models for biomedical research (pp. 425-436). Humana Press: Totowa, NJ, US.

Mani, A. M., Fenwick, M. A., Cheng, Z., Sharma, M. K., Singh, D., \& Wathes, D. C. (2010). IGF1 induces up-regulation of steroidogenic and apoptotic regulatory genes via activation of phosphatidylinositol-dependent kinase/AKT in bovine granulosa cells. Reproduction, 139(1), 139-151.

Matsuo, M., Sumitomo, K., Ogino, C., Gunji, Y., Nishimura, R., \& Hishinuma, M. (2017). Three-step in vitro maturation culture of bovine oocytes imitating temporal changes of estradiol-17 $\beta$ and progesterone concentrations in preovulatory follicular fluid. Archives animal breeding, 60(4), 385-390.

McGee, E. A., \& Hsueh, A. J. (2000). Initial and cyclic recruitment of ovarian follicles. Endocrine reviews, 21(2), 200-214.

Mikkelsen, A. L., \& Lindenberg, S. (2001). Morphology of in-vitro matured oocytes: impact on fertility potential and embryo quality. Human reproduction, 16(8), 1714-1718.

Miller, W. L., Agrawal, V., Sandee, D., Tee, M. K., Huang, N., Choi, J. H., Morrissey, K., \& Giacomini, K. M. (2011). Consequences of POR mutations and polymorphisms. Molecular and cellular endocrinology, 336(1-2), 174-179.

Nagano, M., Katagiri, S., \& Takahashi, Y. (2006). Relationship between bovine oocyte morphology and in vitro developmental potential. Zygote, 14(1), 53-61.

Pan, Z., Zhang, J., Lin, F., Ma, X., Wang, X., \& Liu, H. (2012). Expression profiles of key candidate genes involved in steroidogenesis during follicular atresia in the pig ovary. Molecular biology reports, 39(12), 10823-10832.

Pavlok, A. I., Lucas-Hahn, A., \& Niemann, H. (1992). Fertilization and developmental competence of bovine oocytes derived from different categories of antral follicles. Molecular reproduction and development, 31(1), 63-67.

Payne, A. H., \& Hales, D. B. (2004). Overview of steroidogenic enzymes in the pathway from cholesterol to active steroid hormones. Endocrine reviews, 25(6), 947-970.

Quesnel, H., Pasquier, A., Mounier, A., \& Prunier, A. (1998). Influence of feed restriction during lactation on gonadotropic hormones and ovarian development in primiparous sows. Journal of animal science, 76(3), 856-863.

Rienzi, L., Vajta, G., \& Ubaldi, F. (2010). Predictive value of oocyte morphology in human IVF: a systematic review of the literature. Human reproduction update, 17(1), 34-45.

Ronen-Fuhrmann, T., Timberg, R., King, S. R., Hales, K. H., Hales, D. B., Stocco, D. M., \& Orly, J. (1998). Spatio-temporal expression patterns of steroidogenic acute regulatory protein (StAR) during follicular development in the rat ovary. Endocrinology, 139(1), 303-315.

Seneda, M. M., Esper, C. R., Garcia, J. M., de Oliveira, J. A., \& Vantini, R. (2001). Relationship between follicle size and ultrasound-guided transvaginal oocyte recovery. Animal reproduction science, 67(1-2), 37-43.

Soede, N., Langendijk, P., \& Kemp, B. (2011). Reproductive cycles in pigs. Animal reproduction science, 124(3-4), 251-258.

Stocco, C., Baumgarten, S. C., Armouti, M., Fierro, M. A., Winston, N. J., Scoccia, B., \& Zamah, A. M. (2017). Genome-wide interactions between FSH and insulin-like growth factors in the regulation of human granulosa cell differentiation. Human reproduction, 32(4), 905-914.

Terenina, E., Fabre, S., Bonnet, A., Monniaux, D., Robert-Granié, C., SanCristobal, M., Sarry, J., Vignoles, F., Gondret, F., Monget, P., \& Tosser-Klopp, G. (2016). Differentially expressed genes and gene networks involved in pig ovarian follicular atresia. Physiological genomics, 49(2), 67-80.

Tilly, J., Kowalski, K., Schomberg, D., \& Hsueh, A. (1992). Apoptosis in atretic ovarian follicles is associated with selective decreases in messenger ribonucleic acid transcripts for gonadotropin receptors and cytochrome P450 aromatase. Endocrinology, 131(4), 1670-1676. 
Tu, F., Pan, Z., Yao, Y., Liu, H., Liu, S., Xie, Z., \& Li, Q. (2014). miR-34a targets the inhibin beta B gene, promoting granulosa cell apoptosis in the porcine ovary. Genetics and molecular research 13(2), 2504-2512.

Wang, Q., \& Sun, Q.-Y. (2006). Evaluation of oocyte quality: morphological, cellular and molecular predictors. Reproduction, fertility and development, 19(1), 1-12.

Westhof, G., Westhof, K. F., Braendle, W. L., \& diZerega, G. S. (1991). Differential steroid secretion and gonadotropin response by individual tertiary porcine follicles in vitro. Possible physiologic role of atretic follicles. Biology of reproduction, 44(3), 461-468.

Wolfe, A., Divall, S., \& Wu, S. (2014). The regulation of reproductive neuroendocrine function by insulin and insulin-like growth factor-1 (IGF-1). Frontiers in neuroendocrinology, 35(4), 558-572.

Xia, P., Tekpetey, F. R., \& Armstrong, D. T. (1994). Effect of IGF-I on pig oocyte maturation, fertilization, and early embryonic development in vitro, and on granulosa and cumulus cell biosynthetic activity. Molecular reproduction and development, 38(4), 373-379.

Yang, H., Foxcroft, G., Pettigrew, J., Johnston, L., Shurson, G., Costa, A., \& Zak, L. (2000). Impact of dietary lysine intake during lactation on follicular development and oocyte maturation after weaning in primiparous sows. Journal of animal science, 78(4), 993-1000.

Yuan, Y., Van Soom, A., Leroy, J., Dewulf, J., Van Zeveren, A., de Kruif, A., \& Peelman, L. (2005). Apoptosis in cumulus cells, but not in oocytes, may influence bovine embryonic developmental competence. Theriogenology, 63(8), 2147-2163.

Yuan, B., Liang, S., Jin, Y.-X., Kwon, J.-W., Zhang, J.-B., \& Kim, N.-H. (2016a). Progesterone influences cytoplasmic maturation in porcine oocytes developing in vitro. PeerJ, 4, e2454.

Yuan, H.-J., Han, X., He, N., Wang, G.-L., Gong, S., Lin, J., Gao, M., \& Tan, J.-H. (2016b). Glucocorticoids impair oocyte developmental potential by triggering apoptosis of ovarian cells via activating the Fas system. Scientific reports, 6, 24036.

\section{Supplemental Material}

Supplemental Table S4.1 - Follicular fluid steroid concentrations of high-COC-health sows ( $\geq 70 \%$ healthy COCs; $\mathrm{N}=14)$ vs. low-COC-health sows ( $<70 \%$ healthy COCs; $\mathrm{N}=15)$ on follicular fluid steroid concentrations $(\mathrm{ng} / \mathrm{ml})$.

\begin{tabular}{|c|c|c|c|}
\hline Steroid & low-COC-health & high-COC-health & P-value \\
\hline Pregnenolone $^{\mathrm{a}}$ & $33.02 \pm 3.61$ & $38.34 \pm 3.56$ & 0.31 \\
\hline DHEA $^{\mathrm{b}}$ & $0.08 \pm 0.02$ & $0.07 \pm 0.02$ & 0.74 \\
\hline Progesterone ${ }^{b}$ & $10.93 \pm 1.47$ & $15.29 \pm 1.47$ & 0.05 \\
\hline $17 \alpha-O H-P r o g e s t e r o n e^{b}$ & $3.28 \pm 0.80$ & $5.46 \pm 0.76$ & 0.07 \\
\hline 4-Androsten-3,17-dione & $1.89 \pm 0.26$ & $1.95 \pm 0.26$ & 0.88 \\
\hline 19-Norandrostenedione & $1.36 \pm 0.33$ & $2.71 \pm 0.32$ & $<0.01$ \\
\hline$\beta$-Testosterone ${ }^{b}$ & $44.54 \pm 9.54$ & $50.88 \pm 9.12$ & 0.64 \\
\hline$\alpha$-Testosterone ${ }^{b}$ & $2.32 \pm 0.43$ & $3.68 \pm 0.44$ & 0.04 \\
\hline$\beta$-Estradiol ${ }^{\mathrm{b}}$ & $3.36 \pm 0.63$ & $5.78 \pm 0.63$ & 0.02 \\
\hline 11-Deoxycortisol ${ }^{\mathrm{b}}$ & $3.00 \pm 0.42$ & $3.17 \pm 0.40$ & 0.78 \\
\hline Cortisol $^{b}$ & $20.50 \pm 2.47$ & $13.17 \pm 2.36$ & 0.05 \\
\hline Cortisone $^{b}$ & $11.23 \pm 1.77$ & $11.92 \pm 1.72$ & 0.79 \\
\hline Cortisol/Cortisone & $0.67 \pm 0.12$ & $1.19 \pm 0.22$ & 0.18 \\
\hline 11-Deoxycorticosterone ${ }^{b}$ & $0.85 \pm 0.12$ & $0.92 \pm 0.11$ & 0.68 \\
\hline
\end{tabular}

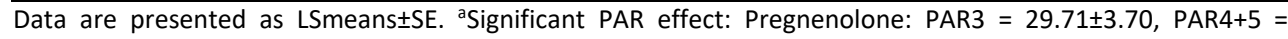
$41.45 \pm 3.31, p=0.02 .{ }^{b}$ Concentration was log transformed to obtain normality. 
Supplemental Table $\mathbf{S 4 . 2}$ - Top regulated genes in sows with high-COC-health $(93 \pm 15 \%$ of COCs healthy $(N=4))$ vs. sows with low-COC-health $(54 \pm 12 \%$ of COCs healthy $(N=4))$.

Top 10 genes with a lower expression in sows with low-COC-health vs. high-COC-health

\begin{tabular}{|c|c|c|c|c|}
\hline Gene symbol $^{\mathrm{a}}$ & Gene description & Accession $\mathrm{nr}$ & FC & FDR \\
\hline INHBB & Inhibin subunit beta B & NM_001164842 & 1.64 & 0.05 \\
\hline TNNI3 & Troponin 13 & NM_001098599 & 1.57 & $<0.01$ \\
\hline ENSSSCT00000010212 & fms-related tyrosine kinase 1 & - & 1.54 & 0.08 \\
\hline MT1A & Metallothionein 1A & NM_001001266 & 1.52 & 0.07 \\
\hline CYP19A2 & Aromatase isoform 2 & NM_214430 & 1.46 & 0.09 \\
\hline ATP6V1E2 & $\begin{array}{l}\text { ATPase } \mathrm{H}+\text { transporting V1 subunit } \\
\text { E2 }\end{array}$ & XM_013996175 & 1.46 & 0.16 \\
\hline CPT1A & Carnitine palmitoyltransferase $1 \mathrm{~A}$ & NM_001129805 & 1.45 & $0 . .01$ \\
\hline CYP19A3 & Aromatase isoform 3 & NM_214431 & 1.43 & 0.09 \\
\hline LOC100512793 & $\begin{array}{l}\text { Cytochrome P450 family } 46 \\
\text { subfamily A member } 1\end{array}$ & - & 1.42 & 0.05 \\
\hline GSTA2 & Glutathione S-transferase A2 & NM_213850 & 1.42 & 0.11 \\
\hline \multicolumn{5}{|c|}{ Top 10 genes with a higher expression in low-COC-health vs. high-COC-health } \\
\hline Gene symbol & Gene description & Accession $\mathrm{nr}$ & FC & FDR \\
\hline DAPL1 & Death-associated protein like 1 & NM_0.001185174 & -1.88 & 0.04 \\
\hline CLDN11 & Claudin 11 & NM_001161641 & -1.84 & 0.12 \\
\hline SOX4 & SRY-box 4 & XM_013977467 & -1.78 & 0.09 \\
\hline NABP1 & Nucleic acid binding protein 1 & NM_001244491 & -1.68 & 0.12 \\
\hline EGLN1 & $\begin{array}{l}\text { Egl-9 family hypoxia inducable } \\
\text { factor }\end{array}$ & XM_021073581 & -1.66 & 0.07 \\
\hline GADD45A & $\begin{array}{l}\text { Growth arrest and DNA damage } \\
\text { inducible alpha }\end{array}$ & NM_001044599 & -1.63 & 0.18 \\
\hline ENSSSCT00000017182 & Unknown & - & -1.61 & 0.03 \\
\hline ABTB1 & $\begin{array}{l}\text { Ankyrin repeat and BTB domain } \\
\text { containing } 1\end{array}$ & XM_013982016 & -1.60 & 0.01 \\
\hline ADM & Adrenomedullin & NM_214056 & -1.60 & 0.18 \\
\hline SEPP1 & Selenoprotein $\mathrm{P}$ & NM_001134823 & -1.56 & 0.02 \\
\hline
\end{tabular}

${ }^{\mathrm{a}}$ For each gene, the fold-change (FC) of high-COC-health sows vs. low-COC-health sows and significance (FDR) are shown. 
Follicular fluid steroid profiling in sows
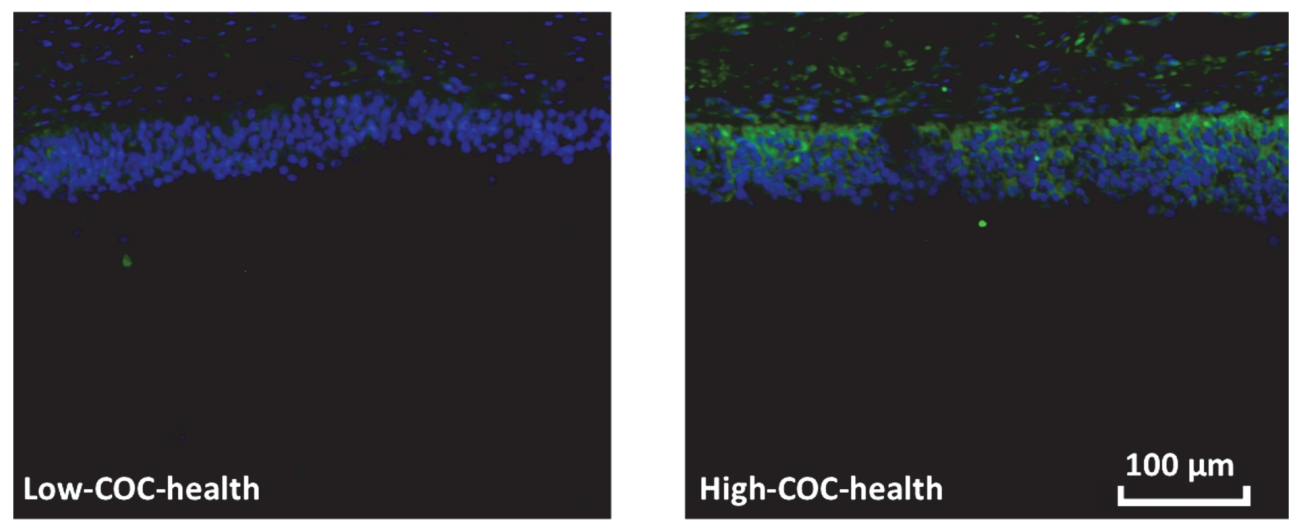

Supplemental Figure S4.1 - Representative pictures of aromatase protein expression in granulosa cells of healthy follicles of sows with low-COC-health $(37 \pm 19 \%$ of COCs healthy $(N=6)$, left) vs. highCOC-health ( $98 \pm 5 \%$ of COCs healthy $(\mathrm{N}=6)$, right) in the smallest and largest follicle of the 10 largest healthy follicles. 


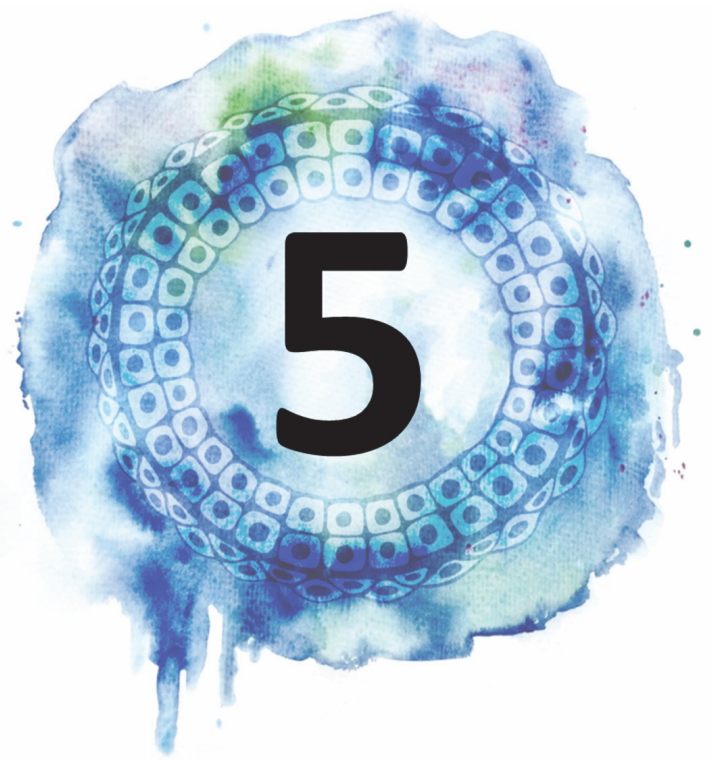

\title{
Steroid profile of porcine follicular fluid and blood serum: relation with follicular development
}

\author{
Natasja G. J. Costermans ${ }^{1}$, Nicoline M. Soede ${ }^{2}$, Marco Blokland ${ }^{3}$, \\ Frederike van Tricht ${ }^{3}$, Jaap Keijer ${ }^{1}$, Bas Kemp ${ }^{2}$ \& Katja J. Teerds ${ }^{1}$ \\ ${ }^{1}$ Human and Animal Physiology, Wageningen University and Research, The Netherlands \\ ${ }^{2}$ Adaptation Physiology Group, Wageningen University and Research, The Netherlands \\ ${ }^{3}$ Wageningen Food Safety Research, Wagenigen University and Research, The Netherlands
}

Revised version published in Physiological Reports 2019 DOI:10.14814/PHY2.14320 



\section{Abstract}

The aim of this study was to identify follicular fluid (FF) steroids which reflect follicular development in the early stages of the follicular phase and to establish whether the levels of these FF steroids correspond to their levels in serum. If these relations are established, serum steroid profiles may be used to monitor follicular development already in this early stage of the follicular phase. We used samples of two experiments, one with multiparous sows at the onset of the follicular phase (weaning) and one with primiparous sows at the mid-follicular phase (48h after weaning). Complete steroid profiles were measured in pooled FF of the 15 largest follicles and serum using high performance liquid chromatography-tandem mass spectrometry. In the multiparous sows at the start of the follicular phase, pooled FF volume, as a measure for average follicle size, tended to be positively related to higher FF $17 \beta$-estradiol levels $(\beta=0.56, p=0.08)$. In primiparous sows at the mid-follicular phase, a larger FF volume was related not only to FF higher $17 \beta$-estradiol levels $(\beta=2.11, p<0.001)$, but also to higher levels of $\beta$-nortestosterone $(\beta=1.15, p<0.0001)$ and its metabolite 19-norandrostenedione $(\beta=1.27, p<0.01)$. In addition, FF volume was related to higher FF $17 \alpha-\mathrm{OH}$-pregnenolone $(\beta=1.63, p=0.03)$ and $17 \alpha-\mathrm{OH}$-progesterone $(\beta=1.83, p<0.001)$, which could indicate that CYP17,20-lyase activity is limiting for $17 \beta$-estradiol production in larger follicles at the beginning of the follicular phase. In serum, most of the steroids were present at lower levels compared to FF, except for the corticosteroids. Serum progestins and androgens were never related to follicle pool volume and steroid levels did not differ in the mid-follicular phase compared to the onset of the follicular phase in the primiparous sows. Serum steroid levels therefore poorly reflect the developmental stage of the follicle pool in the first half of the follicular phase of the oestrus cycle.

Keywords: steroid, follicular fluid, serum, follicle, porcine 


\section{Introduction}

Ovarian follicular fluid (FF) forms the micro-environment in which oocytes develop, and its composition is an important determining factor for successful oocyte development and maturation (reviewed by Li et al., 2008). FF is an exudate of the blood serum and additionally contains growth factors and metabolic intermediates produced by the oocyte and somatic cells composing the follicle (Sugiura et al., 2005; Jiang et al., 2010). Follicular somatic cells, more specifically the theca interna and mural granulosa cells, produce steroids, which are essential in sustaining oocyte and follicle development and survival (Grupen and Armstrong, 2010; Matsuo et al., 2017; Ting et al., 2015). According to the two-cell type theory of ovarian steroidogenesis, theca cells convert cholesterol into progestins and androgens, while granulosa cells express FSH-induced aromatase (CYP19A1) to convert the androgens into 17 $\beta$ estradiol (Liu and Hsueh, 1986). This conversion already starts in early antral follicles. As follicular development continues, follicular estradiol and progesterone levels increase (Liu et al., 2000; McNatty et al., 1975; Van de Wiel et al., 1983) as well as granulosa cell expression of the steroidogenic enzymes CYP19A1 and CYP17A1 (Mahajan, 2008). Studies comparing steroid levels in FF at different stages of the oestrus/menstrual cycle have mainly focused on a limited number of steroids e.g. 17 $\beta$-estradiol, testosterone and progesterone, measured using (radio)immunoassays (e.g. McNatty et al., 1976; van de Wiel et al., 1983; Westergaard et al., 1986). Alternatively, high performance liquid chromatography-tandem mass spectrometry (HPLC-MS/MS) can be used to measure complete steroid profiles. This technique has recently been developed to accurately quantify a large number of steroids simultaneously and with high sensitivity, including their precursors and metabolites (Blokland et al., 2017). Studies using HPLC-MS/MS for FF steroid profiling have focused on FF steroid levels after controlled ovarian hyperstimulation for IVF in humans (Kushnir et al., 2009, 2012, 2016; Naessen et al., 2010; Walters et al., 2019). However, there is limited knowledge of FF steroid profiles and its relation to follicle size in early stages of the follicular phase.

Next to being released into the FF, ovarian steroids are released into the bloodstream, for instance for transport to the brain to elicit the pre-ovulatory LH-surge to induce ovulation, while steroids from the blood may diffuse or be transported towards the follicles. FF and serum show a high correspondence in composition of small molecules and even proteins (Shalgi et al., 1972 and 1973). This suggests similarity between FF and blood serum steroid composition. Next to the ovaries, also the adrenal glands contribute to steroidogenesis via the production of androgen intermediates, progestins and corticosteroids (reviewed by Rainey and Nakamura, 2008), which can also influence follicular development (reviewed by Brann and Mahesh, 1991). However, it is not known if FF and serum steroid composition are correlated, as the few studies that have been performed to assess these relations show conflicting results (e.g. Eiler and Nalbandov 1977; Naskar et al., 2016; Smitz et al., 2006; Walters et al., 2019). If correspondence in serum and FF steroid profiles can be established or 
if serum steroids are related to follicular developmental stages, serum steroid profiles may be used to assess follicular development.

The aim of this study was to identify FF steroids which reflect follicular development in the first half of the follicular phase of the oestrus cycle and to establish whether their FF steroid levels correspond to the levels in serum. To answer our questions, we used sows around weaning from existing studies (Costermans et al., 2019a and 2019b), as sows have a welldefined start of the follicular phase at the end of lactation (reviewed by Soede et al., 2011). Complete steroid profiles of porcine FF and serum were identified using HPLC-MS/MS in order to identify their relation with follicular development and to compare FF and serum steroid levels.

\section{Materials and Methods}

The described experiments were approved by the Animal Care and Use Committee of Wageningen University (DEC2016036 and DEC2017048) and performed according to national and EU guidelines.

\section{Experiment 1: Multiparous sows at onset of the follicular phase}

To assess relations between the steroid profiles in FF and serum and follicular development at the onset of the follicular phase, we used samples of an existing study (Costermans et al., 2019a), with a total of 29 multiparous $\mathrm{N}$-line sows (parity 3 to 5, Norwegian Landrace $x$ Large White; both Topigs Norsvin, Vught, The Netherlands). Briefly, the sows were fed a standard lactation diet (Lacto Excellent, Agrifirm, Apeldoorn The Netherlands) during a 26-day lactation period and weaned on average $12.7 \pm 0.1$ piglets. Immediately after weaning at the onset of the follicular phase, sows were slaughtered by electrical stunning and exsanguination, after overnight fasting, to obtain the ovaries and blood samples. The blood samples were collected in $9 \mathrm{ml}$ serum clot activator tubes (Greiner Bio-One, Monroe, NC, USA).

\section{Experiment 2: Primiparous sows at the mid-follicular phase}

To assess relations between steroid profiles in FF and serum, and follicular development at the mid-follicular phase, and to compare serum steroid profiles at the onset of the follicular phase and mid-follicular phase, we included samples of a second existing study (Costermans et al., 2019b). In short, in this study we used a total of 24 primiparous TN70 sows (Topigs Norsvin) which were either full-fed $(6.5 \mathrm{~kg} /$ day, $\mathrm{N}=12)$ or restricted-fed $(3.25 \mathrm{~kg} /$ day, $\mathrm{N}=12)$ during the last two weeks of a 24-day lactation period. Both groups were fed the same standard lactation diet (Maxima lacto, AgruniekRijnvallei, Wageningen, The Netherlands). Both groups weaned a similar number of piglets ( $12.4 \pm 0.6$ vs. $12.3 \pm 0.6$ piglets for full-fed and restricted-fed, respectively). Following overnight fasting at the time of weaning (onset of the follicular phase), sows were restrained with a nose-sling to collect a blood sample. The sows were slaughtered by stunning and exsanguination 48 hours after weaning (mid-follicular 
phase) to obtain the ovaries and another blood sample again after overnight fasting. Both blood samples were collected in $9 \mathrm{ml}$ serum clot activator tubes (Greiner Bio-One).

\section{Serum and follicular fluid collection}

Serum tubes were kept for 1 hour at $4{ }^{\circ} \mathrm{C}$. Centrifugation took place at $3000 \times \mathrm{g}$ for $10 \mathrm{~min}$ at $4{ }^{\circ} \mathrm{C}$ and stored in $-20^{\circ} \mathrm{C}$ until further analysis. For each sow, the left ovary was placed in plastic bags in a water bath at $37^{\circ} \mathrm{C}$ and the 15 largest follicles were aspirated within 5 hours from collection. These 15 largest follicles were assumed to represent approximately half of the ovulatory follicle pool, as ovulation rates in modern sows are around 25-30 (da Silva et al., 2016). The FF content of the 15 largest follicles was pooled, collected in a tube and allowed to settle for $5 \mathrm{~min}$. The supernatant was removed and centrifuged at $1900 \mathrm{xg}$ for $30 \mathrm{~min}$ at $4{ }^{\circ} \mathrm{C}$ to separate cells from the FF. The total volume of FF of the 15 largest follicles was assessed by reverse pipetting and the FF was subsequently stored at $-80{ }^{\circ} \mathrm{C}$ until further analysis. Pooled FF volume was used as a measure for average follicle size, as average follicle size was assessed using different methods in experiment 1 (Costermans et al., 2019a) and 2 (Costermans et al., 2019b). Pooled FF volume was highly correlated to average follicle size of the 15 largest follicles for both experiments $\left(R^{2}=0.79\right.$ in exp1 and $R^{2}=0.69$ in exp2, both $\left.p<0.0001\right)$.

\section{Steroid profiling}

The pooled FF of the 15 largest follicles of the left ovary and serum samples were used for profiling of endogenously produced steroid hormones. A modified UHPLC-MS/MS method as described in Blokland et al. (2017) was used to detect steroid aglycons in follicular fluid. In short: $900 \mu \mathrm{l}$ water was added to $100 \mu \mathrm{l}$ follicular fluid, followed by solid phase extraction using an Oasis HLB 96-well SPE plate (Waters, Milford, CT, USA); derivatization of aglycons was performed with picolinic acid. Chromatographic separation of the aglycons was performed using a Waters BEH C18 column (Waters) followed by analysis in a Xevo TQS mass spectrometer (Waters) in positive ESI mode.

\section{Statistical analyses}

All statistical analyses were performed using SAS 9.4 (Cary, NC, USA). Normality was tested with Shapiro-Wilk tests using proc UNIVARIATE. The presence of outliers was tested by calculating the studentized residuals using proc REG and when $\geq 3$ residuals were detected, outliers were removed from further analysis. For analysis of differences between steroid concentrations in FF and serum in multiparous sows at the onset of the follicular phase (experiment 1 ) and primiparous sows at the mid-follicular phase (experiment 2), values were not normally distributed and could not be transformed to obtain normality. Differences in steroid concentrations were therefore analysed by Kruskall-Wallis tests using proc NPAR1WAY. Correlations between individual steroid levels in FF and serum were tested separately for experiment 1 and experiment 2 with Spearman rank correlations using proc CORR. 
Differences in serum steroid levels at the onset of the follicular phase and mid-follicular phase (both experiment 2) were assessed by Tobit censored regression truncated at the lower detection limit (proc QLIM), after log transformation to obtain normality. Relations between steroid levels and amount of follicular fluid of the 15 largest follicles, as a measure for average follicle size of the pool, were also assessed by censored regression after values were log transformed to obtain normality. Relations between follicular volume and steroid levels were separately assessed for FF and serum, as many of the serum steroids were not detectable or present in very low levels. For experiment 2, we used a model which included feed level treatment (full-fed and restricted-fed) and the interaction between follicle pool volume and treatment. Interactions between follicle pool volume and treatment were never significant and were therefore removed from the models. Samples of which the steroid levels were below the lower detection limit of the assay were given the value of the lower detection limit (0.01). Steroids of which less than five samples exceeded the lower detection limit of the assay were excluded from further analysis.

\section{Results}

Relations between FF steroid levels and pooled FF volume at the onset of the follicular phase (exp1) and at the mid-follicular phase (exp2)

In multiparous sows at the onset of the follicular phase, average pooled FF volume was $377 \pm 28$ $\mu \mathrm{l}(123-650 \mu \mathrm{l})$. A larger pooled FF volume of the 15 largest follicles was related to higher FF $17 \alpha-\mathrm{OH}$-progesterone and tended to be related to higher FF $17 \beta$-estradiol levels and to higher $17 \alpha-\mathrm{OH}$-pregnenolone (Table 5.1). In primiparous sows at the mid-follicular phase, average pooled FF volume was $280 \mu \mathrm{l}(130-539 \mu \mathrm{l})$ for restricted-fed and $427 \mu \mathrm{l}(184-739 \mu \mathrm{l})$ for fullfed. A larger pooled FF volume was related to higher FF 17ß-estradiol, 19norandrostenedione, $\alpha$-testosterone and $\beta$-nortestosterone levels, and tended to be related to higher $\beta$-testosterone levels. A larger pooled FF volume was also related to higher FF $17 \alpha$ $\mathrm{OH}$-pregnenolone and $17 \alpha-\mathrm{OH}$-progesterone levels (see Table 5.1).

Comparison of FF vs. serum steroid profiles at the onset of the follicular phase (exp 1) and mid-follicular phase (exp2)

FF and serum steroid profiles of multiparous sows at the onset of the follicular phase (experiment 1 ) and primiparous sows at the mid-follicular phase (experiment 2 ) are depicted in Table 5.2. 18 of the 19 steroids were detected in FF on both days (having $\geq 5$ samples above the detection limit) and 12 of 19 and 9 of 19, in serum, respectively. In serum, all progestins, androgens and $17 \beta$-estradiol were either not detectable or were present at lower levels as compared to FF. Only $17 \alpha-\mathrm{OH}$-pregnenolone and $5 \alpha$-androstenedione levels were present at similar levels in FF and serum in the primiparous sows at the mid-follicular phase. As for the corticosteroids; for both experiments, corticosterone levels were lower, while cortisone levels were higher in FF compared to serum. Only in the primiparous sows at the mid-follicular phase of the cycle, FF cortisol levels were lower compared to serum. In both experiments, FF 
cortisol/cortisone ratio was lower compared to serum. Correlations between individual steroids in FF and serum were never significant.

Table 5.1 - Relation between follicular fluid steroid levels $(y, \mathrm{ng} / \mathrm{ml})$ and follicular volume $(x, \mu l)$ of the 15 largest follicles in multiparous sows at the onset of the follicular phase $(N=29$, experiment 1$)$ and primiparous sows at the mid-follicular phase $(\mathrm{N}=24$, experiment 2$)$.

Exp 1: onset of follicular phase Exp 2: mid-follicular phase

\begin{tabular}{lcccccc} 
& $\boldsymbol{\beta}$ & $\mathbf{p}$-value & $\mathbf{N}^{\mathbf{1}}$ & $\boldsymbol{\beta}$ & $\mathbf{p}$-value & $\mathbf{N}^{\mathbf{1}}$ \\
\hline$\beta$-Estradiol & 0.56 & 0.08 & 29 & 2.11 & $<0.001$ & 24 \\
Progesterone & 0.01 & 0.96 & 29 & 0.42 & 0.17 & 24 \\
$17 \alpha$-OH-Progesterone & 1.06 & 0.01 & 28 & 1.83 & $<0.001$ & 23 \\
Pregnenolone & -0.32 & 0.15 & 29 & 0.26 & 0.37 & 24 \\
$17 \alpha$-OH-Pregnenolone & 2.77 & 0.08 & 20 & 1.63 & 0.03 & 10 \\
DHEA & 0.13 & 0.72 & 29 & - & - & 3 \\
19-Norandrostenedione & -0.05 & 0.88 & 29 & 1.27 & $<0.01$ & 24 \\
4-Androsten-3,17-dione & 0.33 & 0.38 & 29 & 0.82 & 0.13 & 23 \\
5 $\alpha$-Androstenedione & - & - & N.D. & -0.25 & 0.67 & 13 \\
$\beta$-Testosterone & 0.42 & 0.21 & 29 & 0.99 & 0.07 & 24 \\
$\alpha$-Testosterone & -0.08 & 0.85 & 29 & 1.39 & $<0.001$ & 23 \\
DHT & - & - & 1 & -0.25 & 0.82 & 20 \\
(5 $\beta, 17 \alpha$-17-Hydroxyandrostan- & 1.17 & 0.65 & 13 & -2.0 & 0.88 & 13 \\
$\beta$-Nortestosterone & -0.06 & 0.89 & 29 & 1.15 & $<0.0001$ & 24 \\
11-Deoxycorticosterone & -0.03 & 0.91 & 29 & -0.92 & 0.21 & 23 \\
Corticosterone & 3.30 & 0.13 & 18 & 0.47 & 0.84 & 11 \\
11-Deoxycortisol & 0.05 & 0.84 & 29 & -1.03 & 0.22 & 23 \\
Cortisol & -0.01 & 0.97 & 29 & -11.97 & 0.35 & 5 \\
Cortisone & -0.11 & 0.74 & 29 & 1.75 & 0.31 & 19 \\
Cortisol/cortisone & 0.01 & 0.98 & 29 & 0.15 & 0.43 & 5 \\
\hline
\end{tabular}

${ }^{1}$ Number of observations above detection limit. Steroids for which $\mathrm{N} \leq 5$ were excluded from further analysis.

${ }^{2}$ Steroid values were not determined in this experiment.

Serum steroid profiles at the onset of the follicular phase vs. mid-follicular phase

Serum steroid profiles in primiparous sows (both full-fed and restricted-fed) at the onset of the follicular phase and at the mid-follicular phase (both experiment 2) are depicted in Table 5.3. Serum progesterone levels tended to be higher $(p=0.06)$ at the mid-follicular phase as compared to the onset of the follicular phase. Other steroids did not differ.

Relations between serum steroid levels and pooled FF volume at the onset of the follicular phase (exp1) and at the mid-follicular phase (exp2)

In serum, at the onset of the follicular phase, a larger pooled FF volume was related to higher 11-deoxycorticosterone levels $(\beta=5.1, p=0.03$ ). No other significant relations between steroid levels and pooled FF volume have been found (Supplemental Table S5.1). 


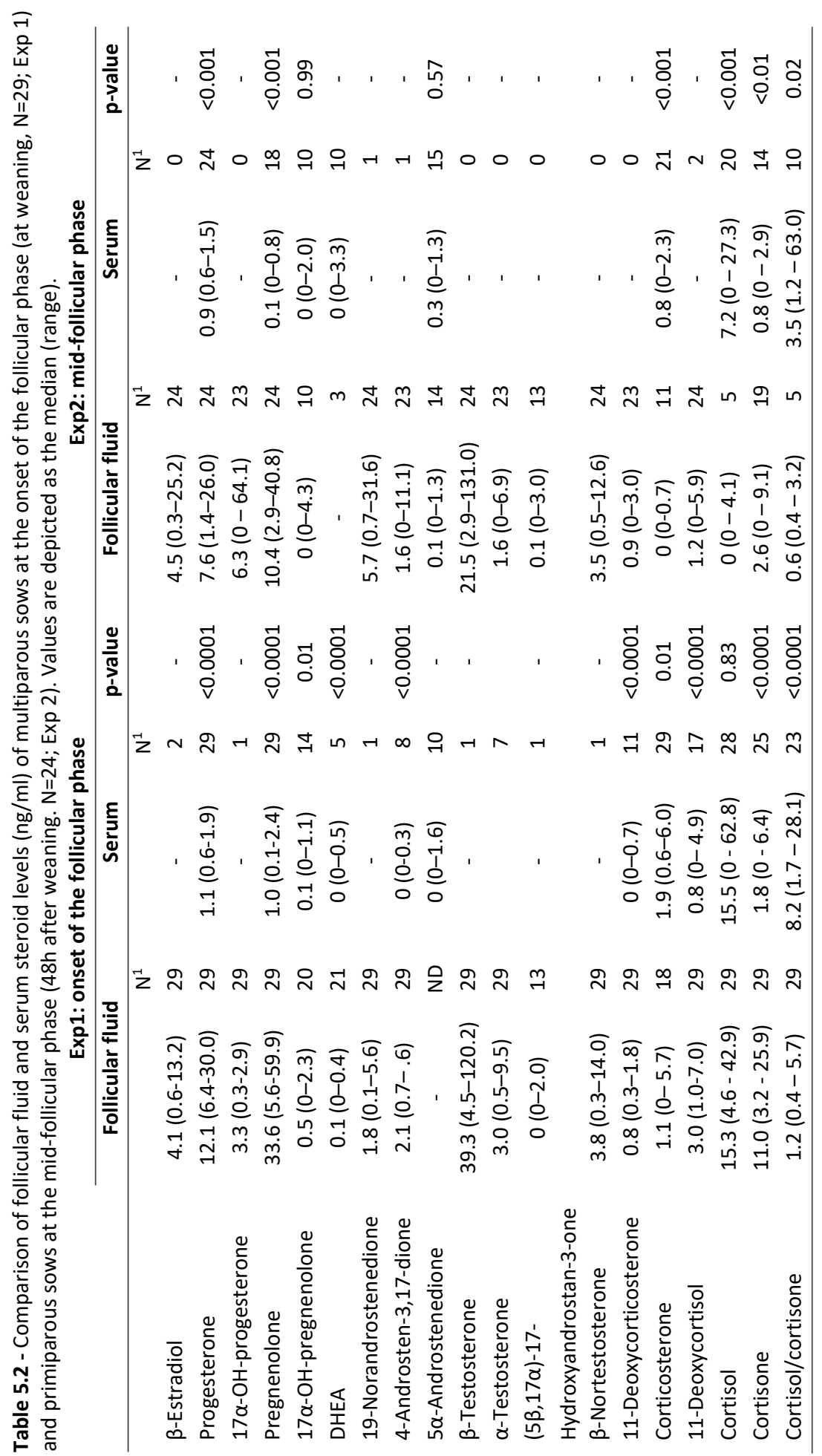


Table 5.3 - Comparison of serum steroid levels $(\mathrm{ng} / \mathrm{ml}$ ) in primiparous sows (both full-fed and restricted-fed) at the onset of the follicular phase (weaning) and at the mid-follicular phase (48h after weaning). Values are depicted as the median (range).

\begin{tabular}{lccccc} 
& \multicolumn{2}{c}{ Onset of the follicular phase } & Mid-follicular phase & & p-value \\
\hline & & $\mathrm{N}^{1}$ & & $\mathrm{~N}^{1}$ & \\
Progesterone & $0.9(0.5-1.4)$ & 24 & $1.0(0.6-1.5)$ & 24 & 0.06 \\
Pregnenolone & $0.2(0-1.3)$ & 20 & $0.1(0-0.8)$ & 19 & 0.55 \\
$17 \alpha-\mathrm{OH}$-pregnenolone & $0(0-3.1)$ & 7 & $0(0-2.0)$ & 11 & 0.26 \\
DHEA & $0(0-4.1)$ & 9 & $0(0-3.3)$ & 10 & 0.65 \\
$5 \alpha$-Androstenedione & - & 4 & $0.3(0-1.3)$ & 16 & - \\
Corticosterone & $0.4(0-1.8)$ & 18 & $0.8(0-2.3)$ & 21 & 0.14 \\
Cortisol & $2.0(0-21.1)$ & 14 & $6.4(0-27.3)$ & 18 & 0.10 \\
Cortisone & $0.9(0-3.1)$ & 15 & $0.6(0-2.9)$ & 14 & 0.74 \\
Cortisol/cortisone & $1.5(0-12.6)$ & 8 & $4.1(1.2-63.0)$ & 9 & 0.53 \\
\hline
\end{tabular}

${ }^{1}$ Number of observations above detection limit. Steroids for which $\mathrm{N} \leq 5$ were excluded from further analysis.

\section{Discussion}

In this study we assessed relations between FF steroid profiles and FF volume of the 15 largest follicles, as measure for average follicle size, in the first half of the follicular phase. To study this, we used samples of a study with multiparous sows at the onset of the follicular phase (Costermans et al., 2019a) and a study with primiparous sows at the mid-follicular phase (Costermans et al., 2019b). Differences in steroid levels between the two experiments cannot be directly attributed to the different developmental stage of the follicle pool as we used sows of a different parity and different sow line, which in general show a different follicular development and ovarian steroid production (Naskar et al., 2016; van Leeuwen et al., 2011; Yun et al., 2018). At the onset of the follicular phase in multiparous sows, a larger pooled FF volume tended to be related to higher $17 \beta$-estradiol levels. At the mid-follicular phase in primiparous sows, pooled FF volume was not only positively related to FF $17 \beta$-estradiol, but also to FF $\beta$-nortestosterone and its metabolite 19-norandrostenedione. The role of these norandrogens in follicular development is not completely clear. It has, however, been suggested that they are produced as part of a minor pathway of androgen aromatization (van Eenoo et al., 2001; Dehennin et al., 1987) and levels are especially high in situations of high estrogen production, such as pregnancy in cows from at least 2 months before partus (de Brabander et al., 1994). Indeed, in our study, FF $\beta$-nortestosterone and 19norandrostenedione were highly correlated to $17 \beta$-estradiol levels $(\beta=0.65, p<0.001$ and $\beta$ $=1.46, p<0.001$, respectively). This could indeed indicate that $\beta$-nortestosterone and 19norandrostenedione were produced alongside $17 \beta$-estradiol in larger follicles in the first half of the follicular phase. As follicular $17 \beta$-estradiol levels increase with follicle size, the production of its steroid precursors increases as well. FF $17 \alpha-\mathrm{OH}$-pregnenolone and $17 \alpha-\mathrm{OH}-$ progesterone levels were higher in sows with a larger pooled FF volume. These $17 \alpha-\mathrm{OH}$ steroids can be converted to DHEA or androstenedione by CYP17A1 which catalyses 17,20- 
lyase activity (reviewed by Miller and Auchus, 2011). In primiparous sows with a 18-day lactation, CYP17,20-lyase activity is low in the early stages of the follicular phase (3d after weaning) and increased 8-fold in theca cells of late pre-ovulatory follicles (Corbin et al., 2003). As CYP17,20-lyase activity was found to be low at the beginning of the follicular phase, its activity could be rate-limiting for $17 \beta$-estradiol production in sows with a larger average follicle size at the onset of the follicular phase and mid-follicular phase. This rate-limiting CYP17,20-lyase activity may be one of the contributing mechanisms to prevent excessive androgen production at the beginning of the follicular phase. Such excessive androgen production is known to inhibit follicular growth and viability (Tarumi et al., 2012) and induces the formation of follicular cysts (Okutsu et al., 2010), similar to what is seen in patients with PCOS (Naessen et al., 2010). Other FF progestins and androgens were not related to pooled FF volume which indicates that the activity of other steroidogenic enzymes such as CYP17 $\alpha$ hydroxylase, needed for the hydroxylation of pregnenolone and progesterone, and CYP19A1, needed for the conversion of androgens into estrogens, are not limiting for $17 \beta$-estradiol production at this stage of follicular development.

In this study we also assessed if, next to FF steroid profiles, serum steroid profiles could reflect follicular development at the beginning of the follicular phase. In both of our experiments, most of the progestins, androgens and $17 \beta$-estradiol are either not detectable or present at very low levels in serum. In later stages of follicular development, serum contains higher levels of $17 \beta$-estradiol which supresses FSH secretion (Henricks et al., 1972; Walters et al., 2019) resulting in the selection of only those follicles which have sufficient FSH and LH receptors for ovulation (reviewed by Knox, 2005). Progesterone production starts to rapidly increase in preovulatory follicles (Walters et al., 2019). This may explain the low levels of serum $17 \beta$-estradiol and progestins in our study as serum samples were obtained from sows at the first half of the follicular phase of the cycle. Next to ovarian androgen production, the adrenal gland also contributes to androgen production, which can serve as precursors for sex steroid production (Kaminska et al., 2000). As androgen levels in serum were very low, our results suggest that the contribution of the adrenal gland to serum steroid levels is probably very limited at the first half of the follicular phase of the cycle.

We used three different methods to assess if serum steroid profiles could be used to monitor follicular developmental stage. We first established whether FF steroid levels correspond to the steroid levels in serum. In both our experiments, cortisol/cortisone was lower in follicular fluid as compared to serum, which is indicative for higher cortisol oxidation and $11 \beta$ hydroxysteroid dehydrogenase (HSD11B) activity in follicles as compared to extra-gonadal tissues. As cortisol can inhibit gonadotropin induced follicular steroidogenesis in cultured granulosa cells (Michael et al., 1993; Hsueh and Erickson, 1978) and negatively influences in vitro oocyte maturation (Yang et al., 1999), it may be postulated that high follicular HSD11B activity in the porcine ovary acts as a protective mechanism to support follicular 
steroidogenesis and also oocyte maturation. This assumption is further supported by a study of Sunak et al. (2007), which found increased cortisol oxidation with increasing antral follicle size, when follicular steroid production is high. Secondly, to further investigate if serum steroid levels are reflective of the follicular developmental stage of the follicle pool, we assessed if serum steroid levels differ at the mid-follicular phase as compared to the onset of the follicular phase in the primiparous sows. However, none of the steroids measured in serum increased from the early to the mid-follicular phase, a phase corresponding with a more developed follicle pool. Finally, as a third method, we assessed if serum steroid levels were related to follicle size and found that relations were (almost) never significant. Taken together, these results show that serum steroids do not reflect follicular development in this first half of the follicular phase.

To summarize, our study describes for the first time a comparison between follicular FF and serum steroid profiles in sows it the first half of the follicular phase. Our analysis revealed that higher follicular $17 \beta$-estradiol production in a follicle pool with larger follicles is accompanied by $\beta$-nortestosterone and 19-norandrostenedione production from the mid-follicular phase onwards. FF $17 \alpha-\mathrm{OH}$-pregnenolone and $17 \alpha-\mathrm{OH}$-progesterone are also related to pooled $\mathrm{FF}$ volume, which indicates that CYP17,20-lyase activity is limiting for $17 \beta$-estradiol production in larger follicles in the early follicular phase. In serum, steroids are generally present in low levels and poorly reflect the developmental stage of the follicle pool in first half of the follicular phase of the cycle.

\section{Acknowledgements}

This research was funded by the Wageningen Institute for Animal Sciences (WIAS) and NWO, by the NWO-WIAS Graduate Programme 2015 grant.

\section{References}

Blokland, M., van Tricht, E., van Ginkel, L., \& Sterk, S. (2017). Applicability of an innovative steroidprofiling method to determine synthetic growth promoter abuse in cattle. The journal of steroid biochemistry and molecular biology, 174, 265-275.

Brann, D. W., \& Mahesh, V. B. (1991). Role of corticosteroids in female reproduction. The FASEB Journal, 5(12), 2691-2698.

Corbin, C., Moran, F., Vidal, J., Ford, J., Wise, T., Mapes, S., Njar, V. C., Brodie, A. M., \& Conley, A. J. (2003). Biochemical assessment of limits to estrogen synthesis in porcine follicles. Biology of reproduction, 69(2), 390-397.

Costermans, N. G. J., Teerds, K. J., Keijer, J., Knol, E., Koopmanschap, R. E., Kemp, B., \& Soede, N. M. (2019a). Follicular development of sows at weaning in relation to estimated breeding value for within-litter variation in piglet birth weight. Animal, 13(3), 554-563.

Costermans, N. G. J., Teerds, K. J., Middelkoop, A., Roelen, B. A. J., Schoevers, E. J., van Tol, H. T. A., Laurenssen, B., Koopmanschap, R. E., Zhao, Y., Blokland, M., van Tricht, F., Zak. L., Keijer, J., Kemp, B., \& Soede, N. M. (2019b). Consequences of negative energy balance on follicular development and oocyte quality in primiparous sows. Biology of reproduction. In press. DOI: 10.1093/biolre/ioz175 
da Silva, C. L. A., Laurenssen, B., Knol, E., Kemp, B., \& Soede, N. (2016). Relationships between ovulation rate and embryonic and placental characteristics in multiparous sows at 35 days of pregnancy. Animal, 10(7), 1192-1199.

de Brabander, H. F., van Hende, J., Batjoens, P., Hendriks, L., Raus, J., Smets, F., Pottie, G., van Ginkel, L., \& Stephany, R. W. (1994). Endogenic nortestosterone in cattle? Analyst, 119(12), 25812585.

Dehennin, L., Jondet, M., \& Scholler, R. (1987). Androgen and 19-norsteroid profiles in human preovulatory follicles from stimulated cycles: an isotope dilution-mass spectrometric study. Journal of steroid biochemistry, 26(3), 399-405.

Eiler, H., \& Nalbandov, A. (1977). Sex steroids in follicular fluid and blood plasma during the oestrus cycle of pigs. Endocrinology, 100(2), 331-338.

Grupen, C. G., \& Armstrong, D. T. (2010). Relationship between cumulus cell apoptosis, progesterone production and porcine oocyte developmental competence: temporal effects of follicular fluid during IVM. Reproduction, fertility and development, 22(7), 1100-1109.

Henricks, D., Guthrie, H., \& Handlin, D. (1972). Plasma estrogen, progesterone and luteinizing hormone levels during the oestrus cycle in pigs. Biology of reproduction, 6(2), 210-218.

Hsueh, A., \& Erickson, G. (1978). Glucocorticoid inhibition of FSH-induced estrogen production in cultured rat granulosa cells. Steroids, 32(5), 639-648.

Jiang, J.-Y., Xiong, H., Cao, M., Xia, X., Sirard, M.-A., \& Tsang, B. K. (2010). Mural granulosa cell gene expression associated with oocyte developmental competence. Journal of ovarian research, $3(1), 6$.

Kaminska, B., Opalka, M., Ciereszko, R., \& Dusza, L. (2000). The involvement of prolactin in the regulation of adrenal cortex function in pigs. Domestic animal endocrinology, 19(3), 147-157.

Knox, R. (2005). Recruitment and selection of ovarian follicles for determination of ovulation rate in the pig. Domestic animal endocrinology, 29(2), 385-397.

Kushnir, M. M., Naessen, T., Kirilovas, D., Chaika, A., Nosenko, J., Mogilevkina, I., Rockwood, A. L., Carlström, K., \& Bergquist, J. (2009). Steroid profiles in ovarian follicular fluid from regularly menstruating women and women after ovarian stimulation. Clinical chemistry, 55(3), 519-526.

Kushnir, M. M., Naessén, T., Wanggren, K., Hreinsson, J., Rockwood, A. L., Meikle, A. W., \& Bergquist, J. (2016). Exploratory study of the association of steroid profiles in stimulated ovarian follicular fluid with outcomes of IVF treatment. The Journal of steroid biochemistry and molecular biology, 162, 126-133.

Kushnir, M. M., Naessén, T., Wanggren, K., Rockwood, A. L., Crockett, D. K., \& Bergquist, J. (2012). Protein and steroid profiles in follicular fluid after ovarian hyperstimulation as potential biomarkers of IVF outcome. Journal of proteome research, 11(10), 5090-5100.

Li, Q., McKenzie, L. J., \& Matzuk, M. M. (2008). Revisiting oocyte-somatic cell interactions: in search of novel intrafollicular predictors and regulators of oocyte developmental competence. Molecular human reproduction, 14(12), 673-678.

Liu, J., Koenigsfeld, A. T., Cantley, T. C., Boyd, C. K., Kobayashi, Y., \& Lucy, M. C. (2000). Growth and the initiation of steroidogenesis in porcine follicles are associated with unique patterns of gene expression for individual componentsof the ovarian insulin-like growth factor system. Biology of reproduction, 63(3), 942-952.

Liu, Y.-X., \& Hsueh, A. J. (1986). Synergism between granulosa and theca-interstitial cells in estrogen biosynthesis by gonadotropin-treated rat ovaries: studies on the two-cell, two-gonadotropin hypothesis using steroid antisera. Biology of reproduction, 35(1), 27-36.

Mahajan, D. K. (2008). "Pig Model to Study Dynamics of Steroids During Ovarian Follicular Growth and Maturation". In Sourcebook of models for biomedical research (pp. 425-436). Humana Press: Totowa, NJ, US.

Matsuo, M., Sumitomo, K., Ogino, C., Gunji, Y., Nishimura, R., \& Hishinuma, M. (2017). Three-step in vitro maturation culture of bovine oocytes imitating temporal changes of estradiol-17 $\beta$ and progesterone concentrations in preovulatory follicular fluid. Archives animal breeding, 60(4), 385-390. 
McNatty, K., Baird, D., Bolton, A., Chambers, P., Corker, C., \& McLean, H. (1976). Concentration of oestrogens and androgens in human ovarian venous plasma and follicular fluid throughout the menstrual cycle. Journal of endocrinology, 71(1), 77-85.

McNatty, K., Hunter, W., McNeilly, A., \& Sawers, R. (1975). Changes in the concentration of pituitary and steroid hormones in the follicular fluid of human graafian follicles throughout the menstrual cycle. Journal of endocrinology, 64(3), 555-571.

Michael, A., Pester, L., Curtis, P., Shaw, R., Edwards, C., \& Cooke, B. (1993). Direct inhibition of ovarian steroidogenesis by Cortisol and the modulatory role of $11 \beta$-hydroxysteroid dehydrogenase. Clinical endocrinology, 38(6), 641-644.

Miller, W. L., \& Auchus, R. J. (2011). The molecular biology, biochemistry, and physiology of human steroidogenesis and its disorders. Endocrine reviews, 32(1), 81-151.

Naessen, T., Kushnir, M. M., Chaika, A., Nosenko, J., Mogilevkina, I., Rockwood, A. L., Carlström, K, Bergquist, J., \& Kirilovas, D. (2010). Steroid profiles in ovarian follicular fluid in women with and without polycystic ovary syndrome, analyzed by liquid chromatography-tandem mass spectrometry. Fertility and sterility, 94(6), 2228-2233.

Naskar, S., Borah, S., Vashi, Y., Thomas, R., Sarma, D., Goswami, J., \& Dhara, S. (2016). Steroid and metabolic hormonal profile of porcine serum vis-à-vis ovarian follicular fluid. Veterinary world, 9(11), 1320.

Okutsu, Y., Itoh, M. T., Takahashi, N., \& Ishizuka, B. (2010). Exogenous androstenedione induces formation of follicular cysts and premature luteinization of granulosa cells in the ovary. Fertility and sterility, 93(3), 927-935.

Rainey, W. E., \& Nakamura, Y. (2008). Regulation of the adrenal androgen biosynthesis. The Journal of steroid biochemistry and molecular biology, 108(3-5), 281-286.

Shalgi, R., Kraicer, P., Rimon, A., Pinto, M., \& Soferman, N. (1973). Proteins of human follicular fluid: the blood-follicle barrier. Fertility and sterility, 24(6), 429-434.

Shalgi, R., Kraicer, P., \& Soferman, N. (1972). Gases and electrolytes of human follicular fluid. Reproduction, 28(3), 335-340.

Smitz, J., Andersen, A. N., Devroey, P., \& Arce, J.-C. (2006). Endocrine profile in serum and follicular fluid differs after ovarian stimulation with HP-hMG or recombinant FSH in IVF patients. Human reproduction, 22(3), 676-687.

Soede, N., Langendijk, P., \& Kemp, B. (2011). Reproductive cycles in pigs. Animal reproduction science, $124(3), 251-258$.

Sugiura, K., Pendola, F. L., \& Eppig, J. J. (2005). Oocyte control of metabolic cooperativity between oocytes and companion granulosa cells: energy metabolism. Developmental biology, 279(1), 20-30.

Sunak, N., Green, D. F., Abeydeera, L. R., Thurston, L. M., \& Michael, A. E. (2007). Implication of cortisol and $11 \beta$-hydroxysteroid dehydrogenase enzymes in the development of porcine (Sus scrofa domestica) ovarian follicles and cysts. Reproduction, 133(6), 1149-1158.

Tarumi, W., Tsukamoto, S., Okutsu, Y., Takahashi, N., Horiuchi, T., Itoh, M. T., \& Ishizuka, B. (2012). Androstenedione induces abnormalities in morphology and function of developing oocytes, which impairs oocyte meiotic competence. Fertility and sterility, 97(2), 469-476.

Ting, A., Xu, J., \& Stouffer, R. (2015). Differential effects of estrogen and progesterone on development of primate secondary follicles in a steroid-depleted milieu in vitro. Human reproduction, 30(8), 1907-1917.

Van de Wiel, D., Bar-Ami, S., Tsafriri, A., \& De Jong, F. (1983). Oocyte maturation inhibitor, inhibin and steroid concentrations in porcine follicular fluid at various stages of the ooestrus cycle. Journal of reproduction and fertility, 68(1), 247-252.

Van Eenoo, P., Delbeke, F. T., de Jong, F. H., \& De Backer, P. (2001). Endogenous origin of norandrosterone in female urine: indirect evidence for the production of 19-norsteroids as byproducts in the conversion from androgen to estrogen. The journal of steroid biochemistry and molecular biology, 78(4), 351-357. 
Van Leeuwen, J., Martens, M., Jourquin, J., Driancourt, M., Kemp, B., \& Soede, N. (2011). Effects of altrenogest treatments before and after weaning on follicular development, farrowing rate, and litter size in sows. Journal of animal science, 89(8), 2397-2406.

Walters, K. A., Eid, S., Edwards, M. C., Thuis-Watson, R., Desai, R., Bowman, M., Marren, A. J., \& Handelsman, D. J. (2019). Steroid profiles by liquid chromatography-mass spectrometry of matched serum and single dominant ovarian follicular fluid from women undergoing IVF. Reproductive biomedicine online, 38(1), 30-37.

Westergaard, L., Christensen, I., \& McNatty, K. (1986). Steroid levels in ovarian follicular fluid related to follicle size and health status during the normal menstrual cycle in women. Human reproduction, 1(4), 227-232.

Yang, J.-G., Chen, W.-Y., \& Li, P. S. (1999). Effects of glucocorticoids on maturation of pig oocytes and their subsequent fertilizing capacity in vitro. Biology of reproduction, 60(4), 929-936.

Yun, J., Björkman, S., Oliviero, C., Soede, N., \& Peltoniemi, O. (2018). The effect of farrowing duration and parity on preovulatory follicular size and oxytocin release of sows at subsequent oestrus. Reproduction in domestic animals, 53(3), 776-783.

\section{Supplemental Material}

Supplemental Table S5.1 - Relation between serum steroid levels $(\mathrm{y}, \mathrm{ng} / \mathrm{ml})$ and follicular volume $(\mathrm{x}$, $\mu \mathrm{l}$ ) of the 15 largest follicles in multiparous sows at the onset of the follicular phase ( $N=29$, experiment 1 ) and primiparous sows at the mid-follicular phase $(\mathrm{N}=24$, experiment 2$)$.

\begin{tabular}{lcccccc} 
& \multicolumn{3}{c}{ Exp 1: onset of follicular phase } & \multicolumn{3}{c}{ Exp 2: mid-follicular phase } \\
\cline { 2 - 7 } & $\boldsymbol{\beta}$ & $\mathbf{p}$-value & $\mathbf{N}^{\mathbf{1}}$ & $\boldsymbol{\beta}$ & $\mathbf{p}$-value & $\mathbf{N}^{\mathbf{1}}$ \\
\hline Progesterone & 0.11 & 0.50 & 29 & 0.07 & 0.60 & 24 \\
Pregnenolone & 0.21 & 0.51 & 29 & 0.59 & 0.60 & 17 \\
17 $\alpha$-OH-pregnenolone & -0.72 & 0.72 & 14 & 3.0 & 0.28 & 10 \\
DHEA & - & - & 4 & -0.38 & 0.92 & 10 \\
5 $\alpha$-Androstenedione & 2.35 & 0.44 & 10 & 0.02 & 0.99 & 15 \\
11-Deoxycorticosterone & 5.1 & 0.03 & 11 & - & - & 0 \\
Corticosterone & 0.19 & 0.56 & 29 & -0.44 & 0.67 & 21 \\
11-Deoxycortisol & 2.69 & 0.24 & 17 & - & - & 2 \\
Cortisol & -0.42 & 0.65 & 28 & 1.95 & 0.34 & 20 \\
Cortisone & 1.49 & 0.22 & 25 & 1.18 & 0.63 & 14 \\
Cortisol/cortisone & -0.25 & 0.60 & 24 & -0.55 & 0.48 & 10 \\
\hline
\end{tabular}

${ }^{1}$ Number of observations above detection limit. Steroids for which $\mathrm{N} \leq 5$ were excluded from further analysis. 



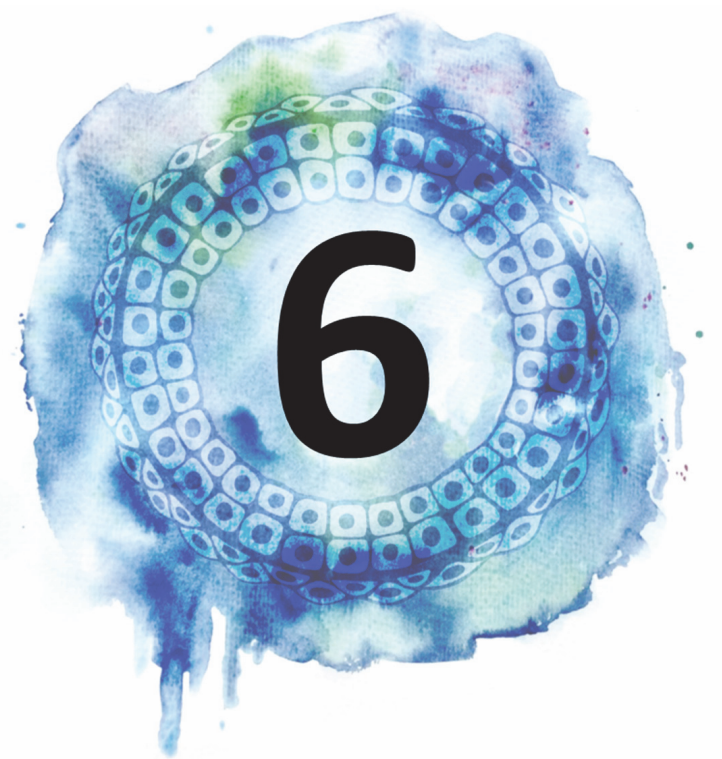

\title{
Consequences of negative energy balance on follicular development and oocyte quality in primiparous sows
}

\begin{abstract}
Natasja G. J. Costermans ${ }^{1,2}$, Katja J. Teerds ${ }^{1}$, Anouschka Middelkoop ${ }^{2}$, Bernard A. J. Roelen ${ }^{3}$, Eric J. Schoevers ${ }^{3}$, Helena T. A. van Tol ${ }^{3}$, Bjorge F. A. Laurenssen ${ }^{2}$, Rudie E. Koopmanschap ${ }^{2}$, Yu Zhao ${ }^{1}$, Marco Blokland ${ }^{4}$, Frederike van Tricht ${ }^{4}$, Louisa Zak ${ }^{5}$, Jaap Keijer ${ }^{1}$, Bas Kemp ${ }^{2}$ \& Nicoline M. Soede ${ }^{2}$

\footnotetext{
${ }^{1}$ Human and Animal Physiology, Wageningen University and Research, The Netherlands

${ }^{2}$ Adaptation Physiology Group, Wageningen University and Research, The Netherlands ${ }^{3}$ Faculty of Veterinary Medicine, Utrecht University, The Netherlands ${ }^{5}$ Topigs Norsvin Research Center B. V., Beuningen, The Netherlands
} ${ }^{4}$ Wageningen Food Safety Research, Wagenigen University and Research, The Netherlands
\end{abstract}





\begin{abstract}
Metabolic demands of modern hybrid sows have increased over the years, which increases the chance that sows enter a substantial negative energy balance (NEB) during lactation. This NEB can influence the development of follicles and oocytes that will give rise to the next litter. To study effects of a lactational NEB on follicular development, we used 36 primiparous sows of which 18 were subjected to feed restriction ( $3.25 \mathrm{~kg} /$ day) and 18 were full-fed ( $6.5 \mathrm{~kg} / \mathrm{day})$ during the last two weeks of a $24.1 \pm 0.3$ day lactation. Feed restriction resulted in a $70 \%$ larger lactational body weight loss and $76 \%$ higher longissimus dorsi depth loss, but similar amounts of backfat loss compared to the full-fed sows. These changes were accompanied by lower plasma insulin-like growth factor 1 (IGF1) and higher plasma creatinine levels in the restricted sows from the last week of lactation onwards. Ovaries were collected $48 \mathrm{~h}$ after weaning. Restricted sows had a lower average size of the 15 largest follicles (-26\%) and cumulus-oocyte complexes showed less expansion after $22 \mathrm{~h}$ in vitro maturation $(-26 \%)$. Less zygotes of restricted sows reached the metaphase stage $24 \mathrm{~h}$ after in vitro fertilization and showed a higher incidence of polyspermy (+89\%). This shows that feed restriction had severe consequences on oocyte developmental competence. Follicular fluid of restricted sows had lower IGF1 (-56\%) and steroids levels (e.g. $\beta$-estradiol, progestins and androgens), which indicated that follicles of restricted sows were less competent to produce steroids and growth factors needed for oocytes to obtain full developmental competence.
\end{abstract}

Keywords: follicle, follicular development, in vitro maturation (IVM), in vitro fertilization (IVF), gonadal steroids, estradiol, insulin-like growth factor, lactation, metabolism, porcine, zygote 


\section{Introduction}

The metabolic state of a female can influence reproductive outcome, which is especially evident in highly productive animal species, such as pigs. Sows usually experience a negative energy balance (NEB) during lactation due to high metabolic demands for milk production, while feed intake is not sufficient to cover the demands for milk production and maintenance (Bergsma et al., 2009). Over the last decades, metabolic demands of modern hybrid sows have increased as litter size and number of piglets weaned have risen. Consequently, sows have a higher chance to enter a substantial NEB during lactation, especially first parity sows that still have a low feed intake capacity (Strathe et al., 2017). This pre-mating NEB can negatively affect reproductive outcome when weight loss exceeds $10-12 \%$ of initial body weight (Thaker et al., 2005), and is especially evident in primiparous sows leading to reduced second parity reproductive performance (Schenkel et al., 2010; Tantasuparuk et al., 2001). High lactational weight loss can lead to a reduction in farrowing rate and litter size (Tantasuparuk et al., 2001; Thaker and Bilkei, 2005). In addition, negative influences on embryonic development and litter uniformity (Hoving et al., 2012; Patterson et al., 2011) and subsequent variation in piglet birth weights (Wientjes et al., 2013) have been reported. The exact origin of reduced reproductive outcome after NEB remains for a part unknown.

The negative effects of the lactational NEB on reproductive outcome can be partly explained by the influence of the pre-mating metabolic state, during and after lactation, on the development of follicles from which oocytes will give rise to the next litter as reviewed by Prunier and Quesnel (2000). For instance, feed restriction during lactation resulted in a smaller follicle size at weaning and 48h post-weaning (Quesnel et al., 1998a) and decreased oocyte maturation rates when oocytes were isolated $38 \mathrm{~h}$ before the anticipated onset of oestrus (Zak et al., 1997). Nevertheless, research investigating relations between the pre-mating metabolic state and follicular and oocyte competence in modern hybrid sows, which experience higher metabolic demands during lactation, is lacking.

The aim of this study is to determine effects of lactational feed intake level and metabolic state on post-weaning follicular and oocyte developmental competence in modern hybrid sows. This was done by subjecting sows to feed restriction during the last two weeks of their first lactation. The lactational metabolic state was assessed as well as follicular and oocyte developmental competence $48 \mathrm{~h}$ after weaning.

\section{Material and methods}

The experiment was approved by the Animal Care and Use Committee of Wageningen University (DEC2017048) and performed according to national and EU guidelines at research facility CARUS (Wageningen University \& Research, the Netherlands). 


\section{Animals}

A total of 36 TN70 (Large White $\mathrm{x}$ Norwegian Landrace, Topigs Norsvin, Vught, The Netherlands) gilts originated from a single gilt breeding farm in three consecutive batches. The gilts arrived two weeks before farrowing at an average age of $363 \pm 21$ days and were divided over two farrowing rooms. During the final two weeks of gestation, sows were fed twice a day $(07.00 \mathrm{~h}$ and $16.00 \mathrm{~h})$ and received $2.9 \mathrm{~kg} /$ day of a standard gestation diet (Standard sow feed, AgruniekRijnvallei, Wageningen, The Netherlands). Within $72 \mathrm{~h}$ after parturition, piglets were cross fostered to obtain similar litter sizes. During lactation, sows were fed an allocated amount of standard lactation diet (ca. $9.3 \mathrm{MJ} \mathrm{NE} / \mathrm{kg}, 156 \mathrm{~g} / \mathrm{kg} \mathrm{CP}, 8.9 \mathrm{~g} / \mathrm{kg}$ lysine, 7.69 SID lysine; Maxima lacto, AgruniekRijnvallei) divided into three meals a day (07.00h, $13.00 \mathrm{~h}$ and $19.00 \mathrm{~h}$ ). All sows received the same amount of the lactation diet until two weeks before weaning, starting at $2 \mathrm{~kg} /$ day at parturition and increasing with $0.5 \mathrm{~kg} /$ day from day 2 after parturition to reach $6 \mathrm{~kg} /$ day two weeks before weaning, according to the TN70 feeding manual for primiparous sows (personal communication, Louisa Zak, Topigs Norsvin). During the last two weeks of lactation, sows were either full-fed (FF; $6.5 \mathrm{~kg} /$ day; $\mathrm{N}=18$ ) or restrictedfed (RES; $3.25 \mathrm{~kg} /$ day; $\mathrm{N}=18$ ). Their piglets were creep-fed. Sows were allocated to treatments based on body weight, backfat thickness and muscle depth at parturition and treatments were equally distributed over both farrowing rooms. Sows had an average lactation length of $24.1 \pm 0.3$ days for both FF and RES and weaned $12.4 \pm 0.6$ vs. $12.3 \pm 0.6$ piglets for FF and RES, respectively. From the day of weaning all sows received a similar amount of the lactation diet ( $3 \mathrm{~kg} /$ day) until $48 \mathrm{~h}$ after weaning, after which the sows were slaughtered by stunning and exsanguination.

\section{Blood sampling}

Blood samples of overnight fasted sows were taken at the first day of lactation (D1), one week after the start of the feed restriction period (D17), at weaning (D24) and at slaughter (D26), between $06.00 \mathrm{~h}$ and $07.00 \mathrm{~h}$. The sows were restrained with a nose-sling and blood was collected from the jugular vein in $9 \mathrm{ml}$ serum clot activator tubes and in $9 \mathrm{ml}$ EDTA coated tubes (Greiner Bio-One, Monroe, NC, USA) to obtain serum and plasma samples, respectively. The collection tubes were stored on ice. After blood sampling, the EDTA tubes were immediately centrifuged, while the serum tubes were first incubated for $1 \mathrm{~h}$ at $4{ }^{\circ} \mathrm{C}$. Centrifugation took place at $3000 \times g$ for $10 \mathrm{~min}$ at $4{ }^{\circ} \mathrm{C}$. Both plasma and serum samples were stored at $-20{ }^{\circ} \mathrm{C}$ until further analysis.

\section{Body weight, backfat depth, muscle depth}

All sows were weighed and P2 backfat depth and loin muscle depth were measured at arrival (D-16), D1 of lactation, at the start of the feed restriction period (D10), at D17 and at D24 (weaning). Body weight was additionally measured at D26 (slaughter). Backfat and loin muscle depth were measured in triplicate at the right and left $P 2$ positions, which is located $6 \mathrm{~cm}$ from the midline, at the position of the last rib using B-mode ultrasonography with a $6.5 \mathrm{MHz}$ linear 
transducer (Aquilla, Esaote, Genova, Italy), similar to Hoving et al. (2011). Piglets were weighed within $24 \mathrm{~h}$ after parturition and at weaning.

\section{Follicle size}

Immediately after slaughter, the left and right ovary were placed against a ruler and photographed from 3 different sides, to facilitate measurement of the follicle size of all visible antral follicles. Follicle size was determined as the largest macroscopically visible diameter of the follicle and measured from the photographs using ImageJ (version 1.51f, National Institutes of Health, USA).

\section{Follicular fluid and COC collection}

The right ovary was immediately frozen in liquid nitrogen and stored at $-80^{\circ} \mathrm{C}$ until further analysis. Left ovaries were placed in plastic bags in a water bath at $37^{\circ} \mathrm{C}$. The 15 largest follicles of the left ovary were aspirated within $5 \mathrm{~h}$ after collection, using a $21 \mathrm{G} \times 5 / 8$ needle and $1 \mathrm{ml}$ syringe (VWR, Amsterdam, The Netherlands). These are assumed to represent approximately half of the ovulatory follicle pool, as ovulation rates in modern sows are around 25 (da Silva et al., 2016). After recovery of the cumulus-oocyte complexes (COCs), the follicular fluid content of the 15 largest follicles was pooled, collected in a tube and allowed to settle for 5 min. The supernatant was removed and centrifuged at $1900 \times g$ at $4{ }^{\circ} \mathrm{C}$ for $30 \mathrm{~min}$ to separate cells from the follicular fluid. The total volume of follicular fluid was assessed by reverse pipetting and was subsequently stored at $-80{ }^{\circ} \mathrm{C}$ until further analysis. The recovered COCs were used for in vitro maturation (IVM) and in vitro fertilization (IVF). The $\mathrm{COC}$ recovery rate was $64.7 \pm 5.6$ vs. $72.2 \pm 3.9$ for RES and FF, respectively.

\section{IVM and IVF}

IVM and IVF took place at $38.5^{\circ} \mathrm{C}$ in a humidified atmosphere of $5 \% \mathrm{CO}_{2}$ in air. All compounds used to make the media were obtained from Sigma Aldrich, unless otherwise stated. All media were pre-incubated at least $2 \mathrm{~h}$ prior to use. The pool of COCs of each sow was separately collected and transferred to a one-eyed dish in IVM-wash medium which consisted of NCSU23 (Petters and Wells, 1993) supplemented with $0.6 \mathrm{mM}$ cysteine, $1 \mathrm{mM}$ L-glutamine, $25 \mu \mathrm{M}$ $\beta$-mercaptoethanol, $2.2 \mathrm{mg} / \mathrm{ml} \mathrm{NaHCO}, 25 \mathrm{mM}$ unbuffered HEPES and 10\% v/v porcine follicular fluid. This pool of porcine follicular fluid was formed by pooling follicular fluid collected from sows using antral follicles between 3-8 $\mathrm{m}$. Recovered COCs from each sow were transferred to a four-well culture dish, washed once and cultured in IVM-I medium (M199, Gibco BRL, Paisley, UK) supplemented with $2.2 \mathrm{mg} / \mathrm{ml} \mathrm{NaHCO}, 10 \% \mathrm{v} / \mathrm{v}$ porcine follicular fluid, $5.0 \mathrm{mM}$ sodium-pyruvate, $1 \mathrm{mM}$ L-glutamine, $200 \mu \mathrm{M}$ cysteamine, $0.05 \mathrm{IU} / \mathrm{ml} \mathrm{FSH}$ and $1 \%$ pen/strep. Photographs were taken for assessment of COC morphology and COC size. After $22 \mathrm{~h}, \mathrm{COCs}$ were washed once and cultured for another 22h in IVM-II medium (IVM-I medium without FSH). In this system, cumulus expansion was not measurable after $44 \mathrm{~h}$ as the cumulus cells showed a very high degree of expansion. After 44h of IVM, COCs were denuded by repeated pipetting, washed once and incubated for $30 \mathrm{~min}$ in Modified Tris-buffered medium 
(13.1 mM NaCl, $3.0 \mathrm{mM} \mathrm{KCl}, 20.0 \mathrm{mM}$ Tris, $11.0 \mathrm{mM}$ D-glucose, $7.5 \mathrm{mM} \mathrm{CaCl} 2 \cdot 2 \mathrm{H}_{2} \mathrm{O}$ and $5.0 \mathrm{mM}$ Na-Pyruvate) containing $1 \mathrm{mM}$ caffeine, $0.1 \%$ BSA and $1 \%$ pen/strep. One $\mathrm{ml}$ of fresh extended semen originating from 5 different Tempo boars (Topigs Norsvin) was added to $3 \mathrm{ml}$ of IVF-medium and centrifuged at $700 \times \mathrm{g}$ for $5 \mathrm{~min}$ at $25^{\circ} \mathrm{C}$. Supernatant was removed and sperm was washed twice by resuspending the pellet in $8 \mathrm{ml}$ of IVF medium, and centrifuged as described above. Sperm cells were added to the denuded oocytes to obtain a final concentration of $1^{*} 10^{4}$ sperm cells $/ \mathrm{ml}$. The sperm and oocytes were co-incubated for $24 \mathrm{~h}$.

\section{Presumptive zygote staining}

The developmental stage of the presumptive zygotes was assessed $24 \mathrm{~h}$ after IVF using an immunofluorescent staining for 5-methylcytosine and propidium iodide to stain total DNA. The 5-methylcytosine staining was used to better distinguish developmental stages of the zygotes, as methylation patterns change during zygote development [16]. Presumptive zygotes were fixed in $4 \%$ paraformaldehyde for $15 \mathrm{~min}$ and incubated overnight in $1 \%$ paraformaldehyde $+0.2 \%$ Triton. Subsequently, the presumptive zygotes were incubated in $4 \mathrm{~N} \mathrm{HCl}$ for $15 \mathrm{~min}$ and neutralized in $100 \mathrm{mM}$ Tris- $\mathrm{HCl}(\mathrm{pH} \mathrm{8.0)}$ for $10 \mathrm{~min}$. Zygotes were washed $3 \times 5$ min in PBS pH $7.4(+0.1 \% \mathrm{w} / \mathrm{v}$ PVP), blocked in $5 \%(\mathrm{v} / \mathrm{v})$ normal goat serum in PBS (+0.1\% w/v PVP) for $60 \mathrm{~min}$ and incubated with anti-5-mC mouse antibody (39649, clonenr: 33D3, Active Motif, Carlsbad, CA, USA) diluted 1:500 (v/v) in PBS-BSAc (Aurion, Wageningen, The Netherlands) for $60 \mathrm{~min}$. Zygotes were washed $3 \times 5 \mathrm{~min}$ in PBS (+0.1\% PVP) and incubated with secondary goat anti-mouse Alexa Fluor 488 (A-21131, ThermoFisher Scientific, Waltham, MA, USA) diluted 1:200 (v/v) in PBS-BSAc for $60 \mathrm{~min}$ at room temperature. After $3 \times 5$ min washing, zygotes were counterstained with propidium iodide (Sigma Aldrich) diluted in PBS (1:100) for $5 \mathrm{~min}$ and mounted on microscope slides (Menzel- Gläser, Braunschweig, Germany) to assess the developmental stage and methylation status of the presumptive zygotes. Zygotes were imaged at 40 times magnification using a fluorescence microscope (Leica DM6B), a digital camera (DFC365 FX) and imaging software (LasX; all Leica Microsystems, Amsterdam, The Netherlands).

\section{IVM and IVF outcome}

Photographs taken at $\mathrm{Oh}$ and $22 \mathrm{~h}$ after start of IVM were used for COC morphology analysis and COC size measurements. COC morphology was assessed at Oh to determine the number of normal (healthy) and atretic (unhealthy) COCs for each sow, according to Alvarez et al. (2009). Individual COC size was determined and averaged for each sow using ImageJ (National Institutes of Health). The percentage COC expansion was defined as the average COC size at $22 \mathrm{~h}$ divided by the average $\mathrm{COC}$ size at $0 \mathrm{~h}$. The developmental stage of the presumptive zygotes was studied $24 \mathrm{~h}$ after IVF and individual zygotes were classified according to pronuclear stages as described in Wossidlo et al. (2011). Classifications used were, pro-nuclear stage 0 (PN0), pronuclear stage 1-5 (PN1-5), metaphase and G2 (2-cell stage). The percentage fertilized oocytes was defined for each sow by dividing the number of zygotes from the 
pronucleus stage onwards by the total number of COCs. Percentage polyspermy was determined by dividing the number of fertilized zygotes with 3 or more pronuclei by the total number of zygotes. IVM and IVF results of two RES sows were excluded from analysis since fewer than 5 COCs were successfully isolated.

\section{Follicular fluid steroid profiling}

A representative subset of $12 \mathrm{FF}$ and 12 RES sows were chosen based on average weight loss during lactation and average follicle size and used for follicular fluid endogenous steroid hormone profiling. The pooled follicular fluid of the 15 largest follicles of the left ovary was used. A slightly modified sample clean-up and mass spectrometric detection method as described in Blokland et al. (2017) was used to detect endogenous aglycons in follicular fluid. The sample clean-up consist of mixing $900 \mu$ l water with $100 \mu$ follicular fluid after which the diluted sample is passed through an Oasis HLB 96-well solid phase extraction plate (Waters, Milford, CT, USA). To enhance the sensitivity of the mass spectrometric method derivatization of aglycons was performed with picolinic acid. The aglycons were separated by chromatograpy on a Waters $\mathrm{BEH} \mathrm{C}_{18}$ column (Waters) followed by analysis on a Xevo TQ-S mass spectrometer (Waters) in positive ESI mode.

\section{Blood serum and plasma measurements}

All assay procedures were performed according to manufacturer's instructions, unless stated otherwise. All analyses were performed in duplicate and only samples with an intra-assay CV $\leq 15 \%$ were included, which resulted in the exclusion of a few samples. Plasma insulin and leptin concentrations were measured using a radioimmunoassay kit (Porcine Insulin PI-12K and Multi-Species Leptin XL-85K, respectively, EMD Millipore corporation, Billerica, MA, US). Plasma insulin-like growth factor 1 (IGF1) was measured with an immunoradiometric assay (A15729, Beckman Coulter, Woerden, The Netherlands) according to the manufacturer's protocol supplemented with additional acid-ethanol extraction $(87.5 \% \mathrm{v} / \mathrm{v}$ EtOH and $2.9 \%$ $\mathrm{v} / \mathrm{v} 12 \mathrm{~N} \mathrm{HCl}$ ). An enzymatic calorimetric test was used to measure plasma creatinine (Creatinine PAP FS, DiaSys Diagnostic Systems $\mathrm{GmbH}$, Holzheim, Germany) and serum nonesterified fatty acid (NEFA) (NEFA-HR(2) kit, Wako Chemicals, Neuss, Germany). For the NEFA measurement, different from the manufacturer's protocol, $5 \mu \mathrm{l}$ serum was added to the plate and $100 \mu \mathrm{l}$ of reagent 1 was added to the wells and incubated for $10 \mathrm{~min}$ at $37^{\circ} \mathrm{C}$. Subsequently, $50 \mu \mathrm{l}$ of reagent 2 was added followed by another incubation step of $10 \mathrm{~min}$ at $37^{\circ} \mathrm{C}$.

Follicular fluid IGF1

Follicular fluid IGF1 levels were measured similar to plasma IGF1. 


\section{$\underline{\text { Statistical analyses }}$}

Distributions of the means and residuals were examined to verify model assumptions of normality and homogeneity of variance. Insulin, pregnenolone, $\beta$-testosterone, 4-androsten3-17-dione and $\beta$-nortestosterone levels were log transformed to obtain normality. The presence of outliers was tested by calculating the studentized residuals using proc REG (SAS 9.4, Cary, NC, USA) and outliers were excluded from further analysis. Follicular and metabolic differences between the treatment groups FF and RES were analysed using proc MIXED in SAS 9.4 (Cary, NC) in models that also contained batch as a random effect. A correction for repeated measures (day of measurement) was added to the model for analysis of body weight, backfat depth, muscle depth and serum and plasma measurements. Additionally, relations between metabolic parameters and follicular or oocyte parameters were estimated using the model: $Y_{i j}=\mu+$ treatment $_{i}+\beta i X_{i j}+\beta_{i} X_{i j}{ }^{*}$ treatment $+\varepsilon_{i j}$, where $Y_{i j}$ is the dependent variable and either a metabolic or follicular or oocyte parameter, $\beta$ is the regression coefficient and $X_{i j}$ is one of the metabolic or follicular parameters. The interactions were excluded from the models when not significant. All values are presented as LS means $\pm \mathrm{SE}$.

\section{Results}

Feed intake, metabolic state, and litter growth

The realised feed intake was indeed lower for the RES sows and higher in the FF sows during the feed restriction period (Fig. 6.1). All sows lost body weight during lactation, but RES sows showed a higher percentage loss of their initial body weight $(19.6 \pm 1.4$ vs. $11.5 \pm 1.4 \%$, $\mathrm{p}<0.0001)$ and higher muscle depth loss $(1.7 \pm 0.1 \mathrm{vs} .1 .1 \pm 0.1 \mathrm{~mm}, \mathrm{p}=0.01)$ during lactation as compared to FF sows. RES and FF sows lost a similar amount of backfat during lactation (5.1 \pm 0.6 vs. $4.6 \pm 0.6 \mathrm{~mm}$ for RES and FF, respectively) (Fig. 6.2).

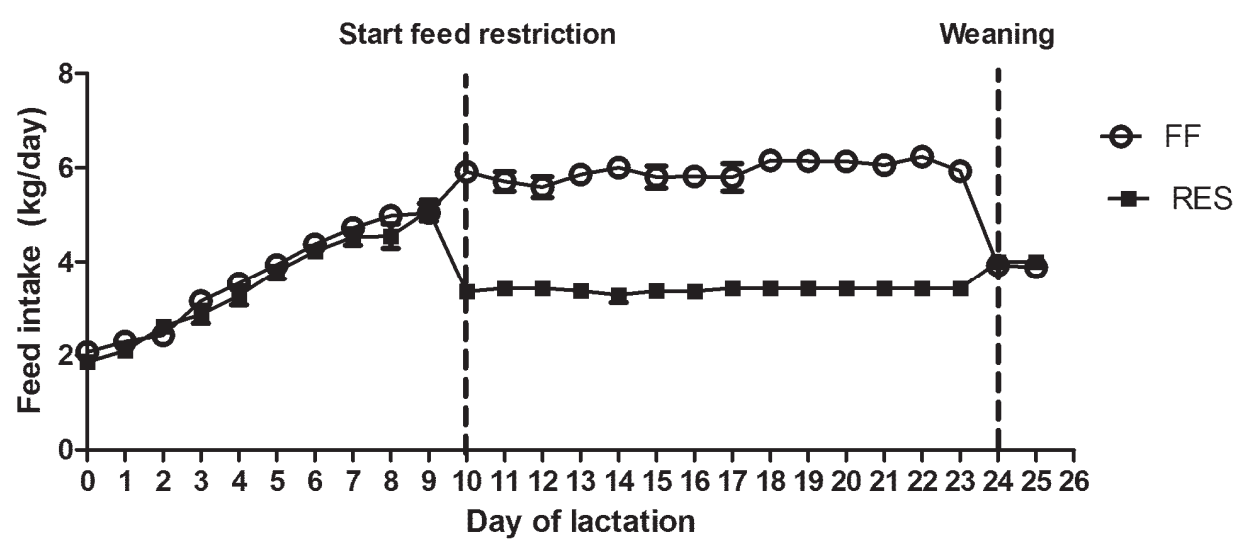

Figure 6.1 - Average daily feed intake (kg/day) of full-fed (FF) and restricted-fed (RES) primiparous sows. All sows received a similar gradually increasing level of a standard lactation diet up to $6 \mathrm{~kg} / \mathrm{day}$ at day 10 of lactation. From day 10 until weaning at day 24 , sows either received $6.5 \mathrm{~kg} /$ day or 3.25 $\mathrm{kg} /$ day, respectively. After weaning, all sows received $4 \mathrm{~kg} /$ day of the lactation diet. 
Within treatment groups, body weight, muscle depth and backfat depth significantly decreased during lactation at all measured time-points, except for muscle depth between arrival and D1 in the FF sows. Litter growth during lactation was reduced in the litters of the RES sows $(56.2 \pm 3.9$ vs. $66.2 \pm 3.9 \mathrm{~kg}, \mathrm{p}<0.01)$ compared to FF. To further assess the metabolic state of the sows, plasma creatinine, IGF1, insulin, leptin and serum NEFA levels were measured. Plasma creatinine and IGF1 levels were higher for FF sows at D17 (1 week after start feed restriction), D24 (weaning) and D26, basal insulin levels were lower for FF sows at D24. Plasma leptin and serum NEFA levels did not differ between FF and RES sows at any of the measured time-points (Fig. 6.3).
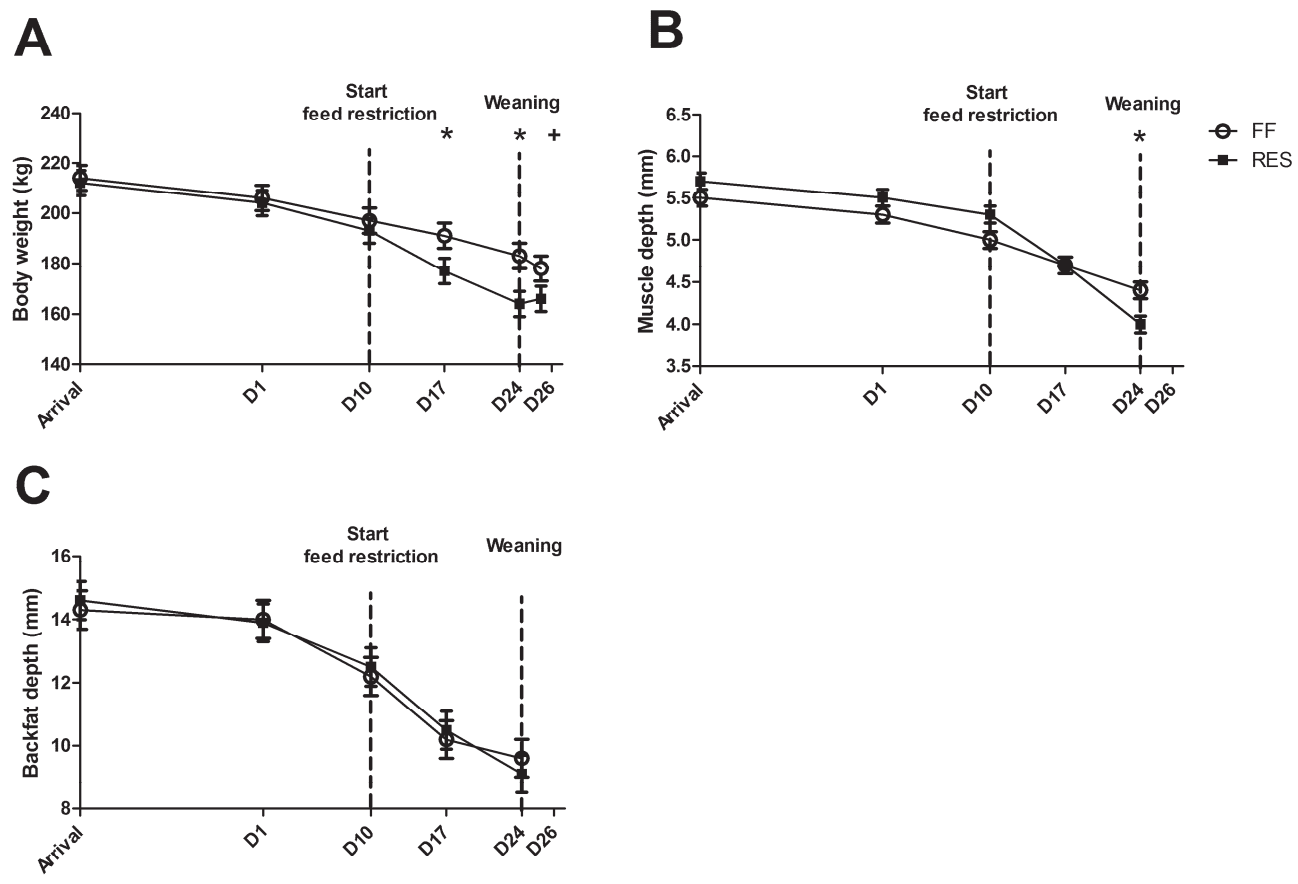

Figure 6.2 - (A) Average body weight (kg), (B) longissimus dorsi muscle depth ( $\mathrm{mm}$ ) and (C) backfat depth $(\mathrm{mm})$ for full-fed (FF) and restricted-fed (RES) primiparous sows which received either $6.5 \mathrm{~kg} / \mathrm{day}$ or $3.25 \mathrm{~kg} /$ day for the last two weeks of lactation. Body weight, loin muscle and backfat depth were measured at arrival, the first day of lactation (D1), at the start of the feed restriction period (FR) (D10), one week after start of FR (D17) and at weaning (D24). Body weight was additionally measured at slaughter (D26). * P-value $<0.05,+$ P-value $>0.05$ and $<0.10$. Parameters were always significantly different between days within treatment $(P<0.05)$, except for muscle depth at arrival and D1 for the FF sows. 

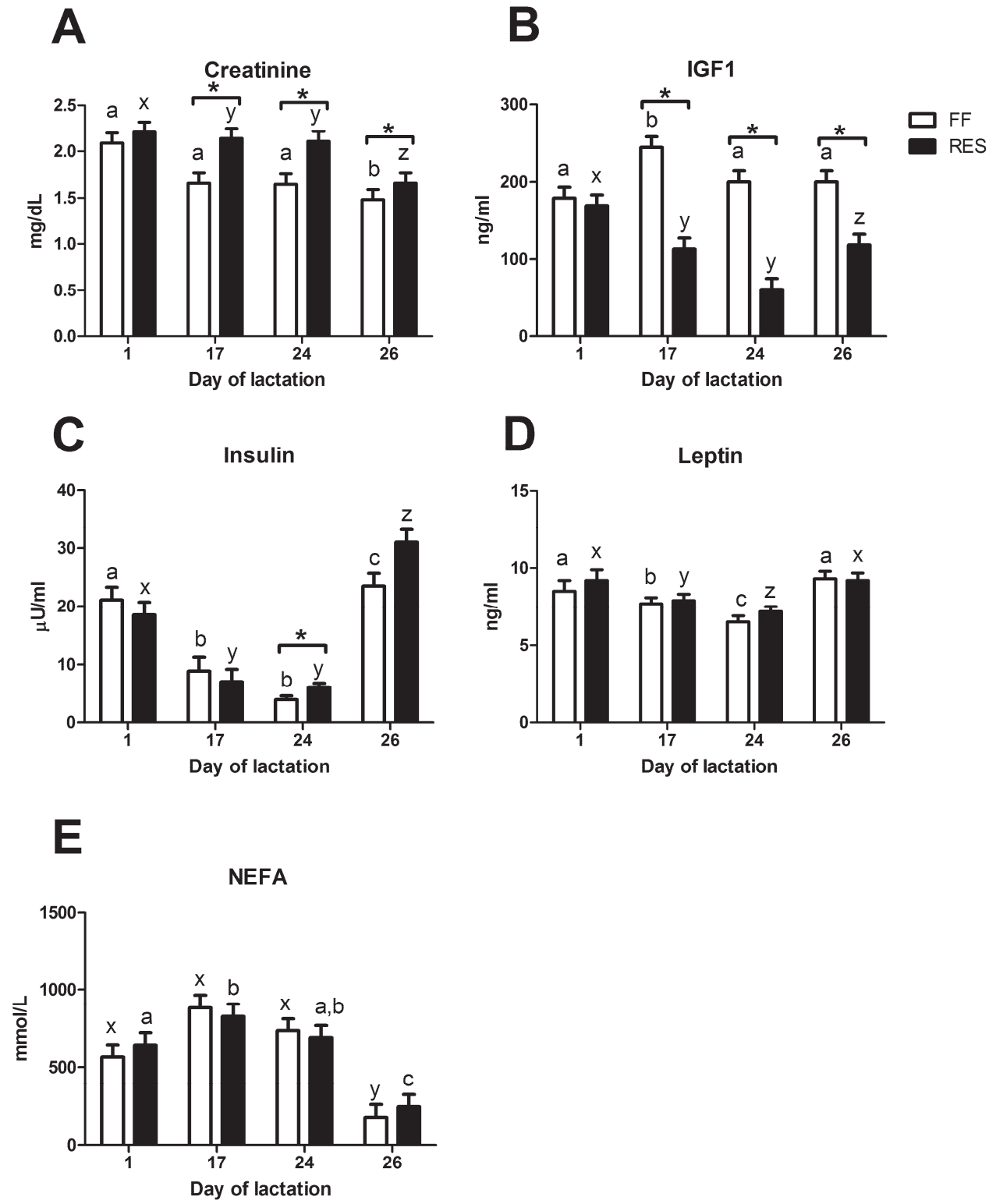

Figure 6.3 - Fasted plasma (A) creatinine (B) IGF1 (C) basal insulin (D) leptin and (E) serum NEFA levels of full-fed (FF) and restricted-fed (RES) primiparous sows which received either $6.5 \mathrm{~kg} / \mathrm{day}$ or 3.25 $\mathrm{kg} /$ day for the last two weeks of lactation. Measurements were done at the first day of lactation (D1), one week after start of the feed restriction period (D17), at weaning (D24) and at slaughter (D26). *Pvalue $<0.05$. The effect of measurement day for each parameter are depicted above the figures. Different letters indicate a significant difference between days within a treatment (abc for FF and $x y z$ for RES). 


\section{Follicle and oocyte developmental competence}

Total number of visible antral follicles in different size categories of both the left and right ovary is shown in Fig. 6.4A. Average follicle size of the 15 largest follicles of both the left and right ovary was lower for RES as compared to FF sows (Fig. 6.4B). The percentage healthy COCs, as measured at the start of IVM, was similar between the two groups (Fig. 6.4C). However, average $\mathrm{COC}$ size at $0 \mathrm{~h}$ was smaller and cumulus expansion was reduced after $22 \mathrm{~h}$ of IVM in RES compared to FF (Fig. 6.4C). Oocytes of RES sows tended to have lower fertilization rates $(p=0.10)$ and zygotes showed a higher incidence of polyspermy $24 \mathrm{~h}$ after start of IVF (Fig. 6.4C). Fewer of the developing zygotes from RES sows were at the metaphase stage of the first division $24 \mathrm{~h}$ after IVF, as compared to zygotes from FF sows (Fig. 6.5). Combined these results suggest a poorer developmental competence of the oocytes that is related to the severity of the negative energy balance during lactation.
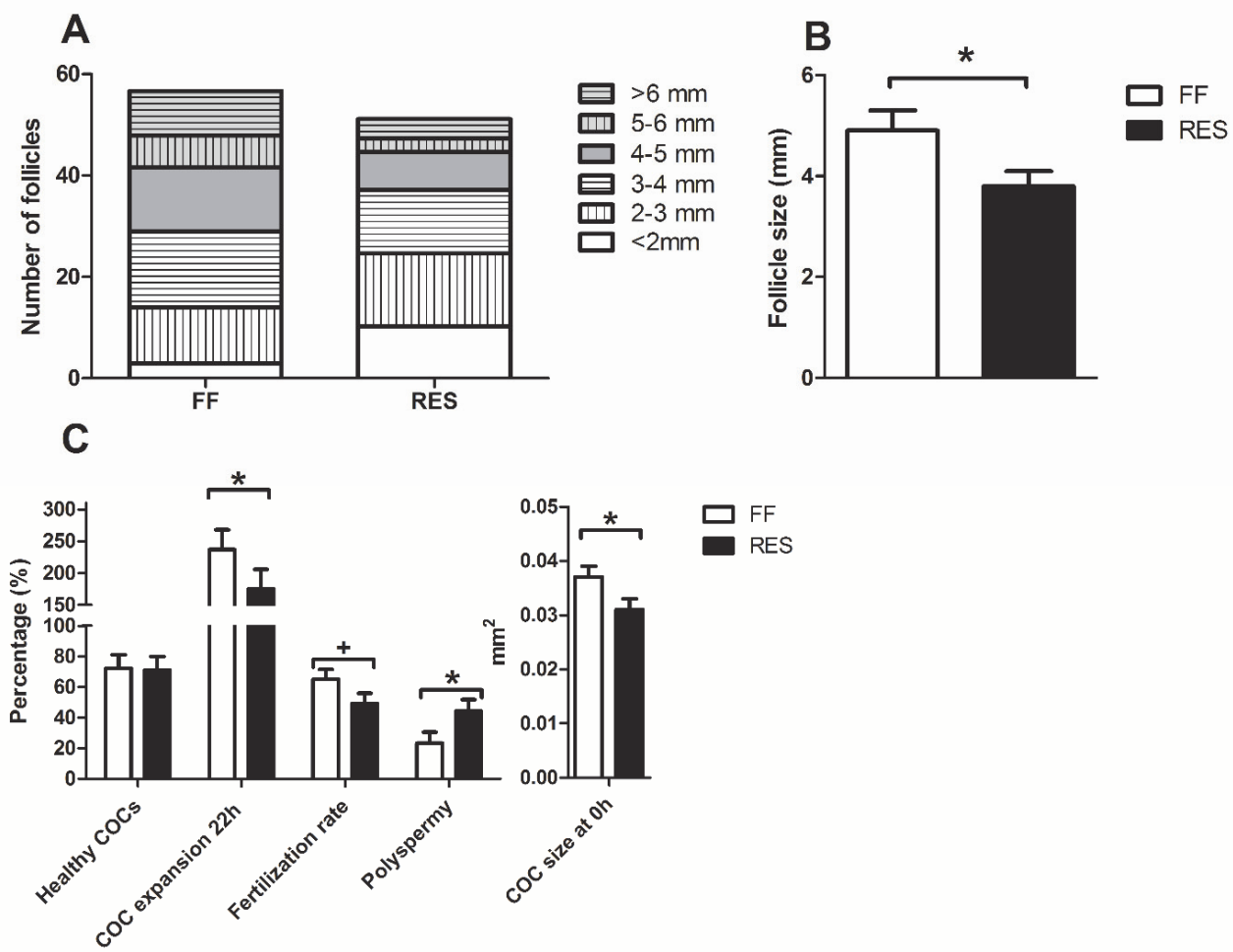

Figure 6.4 - (A) Total number of visible antral follicles in different size categories of both the left and right ovary (B) Average follicle size of the 15 largest follicles of both the left and right ovary $48 \mathrm{~h}$ after weaning and (C) percentage healthy cumulus-oocyte complexes (COCs), average $\mathrm{COC}$ size at $0 \mathrm{~h}$, percentage COC expansion at $22 \mathrm{~h}$ and fertilization rate of the successfully isolated COCs of the 15 largest follicles of the left ovary and polyspermy (\%) of the fertilized oocytes for full-fed and restrictedfed sows which received either $6.5 \mathrm{~kg} /$ day or $3.25 \mathrm{~kg} /$ day for the last two weeks of lactation. COCs were collected 48 h after weaning. ${ }^{*}$ P-value $<0.05,+P$-value $\geq 0.05$ and $<0.10$. 


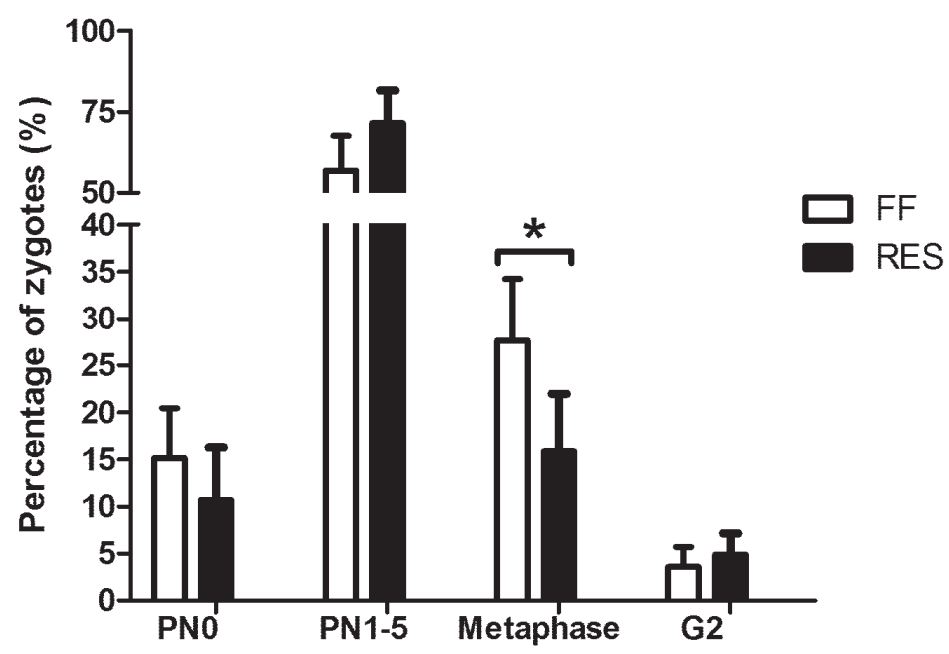

Figure 6.5 - Developmental stage of the presumptive zygotes $24 \mathrm{~h}$ after IVF, for full-fed (FF) and restricted-fed (RES) sows which received either $6.5 \mathrm{~kg} /$ day or $3.25 \mathrm{~kg} / \mathrm{day}$ for the last two weeks of lactation. Cumulus-oocyte complexes were collected $48 \mathrm{~h}$ after weaning. PNO = pro-nuclear stage 0 , PN1-5 = pro-nuclear stage 1-5, G2= 2-cell stage, as an average percentage of the total number of zygotes of each animal (total number of zygotes $=6.8 \pm 0.6$ vs. $4.0 \pm 0.6$ for FF and RES, respectively). ${ }^{*} \mathrm{P}-$ value $<0.05$.

Pooled follicular fluid of the 15 largest follicles of RES sows had a lower concentration of $\beta$ estradiol as compared to FF sows and follicular fluid of RES sows also had a lower concentration of pregnenolone and intermediates of the delta 4-pathway of steroid production; progesterone, $17 \alpha-\mathrm{OH}$-progesterone and androstenedione. Intermediates of the delta 5-pathway of steroid production, $17 \alpha-\mathrm{OH}$-pregnenolone, DHEA and androstenediol were not detectable because of low concentrations. Corticosteroid concentrations did not differ between the two groups (Fig. 6.6 and Supplemental Fig. S6.1 for a schematic overview). 


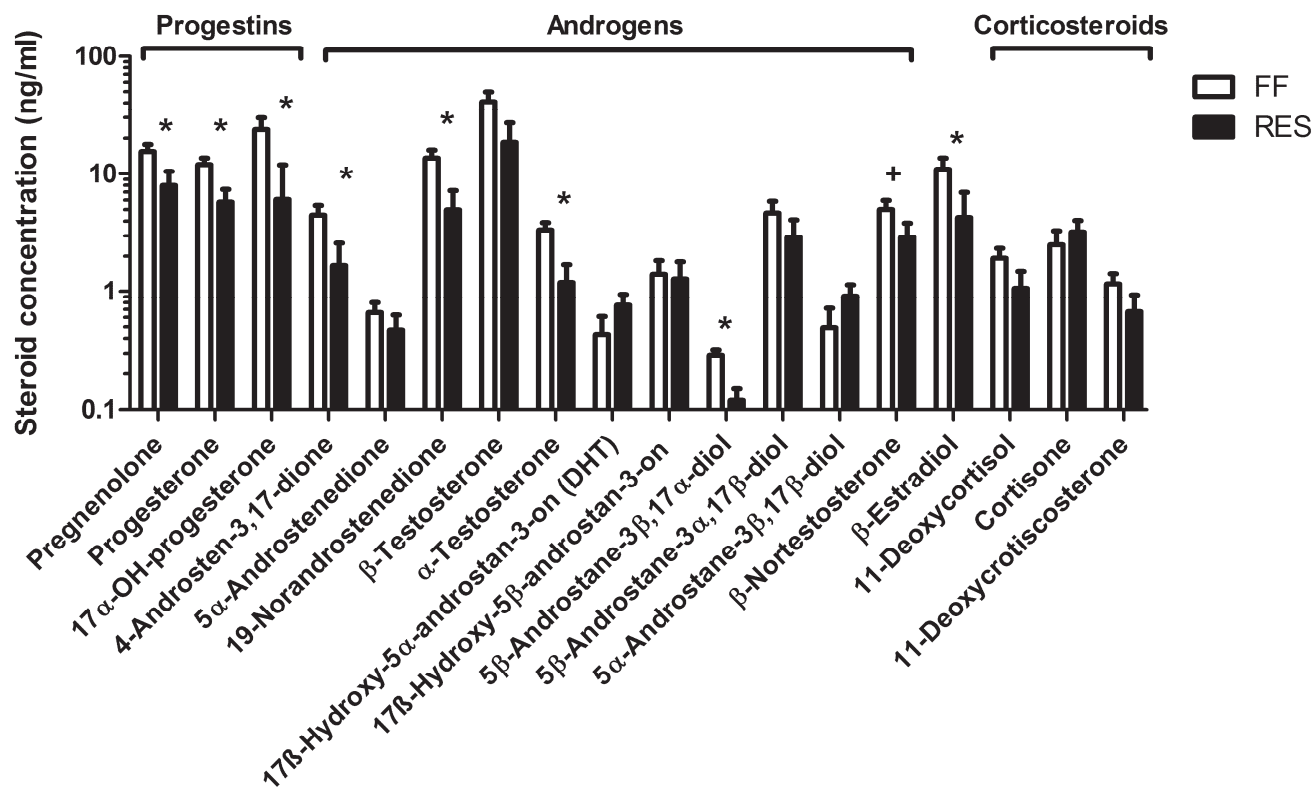

Figure 6.6 - Follicular fluid steroid profile measured $48 \mathrm{~h}$ after weaning, of full-fed (FF) and restrictedfed (RES) sows which received either $6.5 \mathrm{~kg} /$ day or $3.25 \mathrm{~kg} /$ day for the last two weeks of lactation. *Pvalue $<0.05 *$ P-value $<0.05+\mathrm{P}$-value $\geq 0.05$ and $<0.10$.

Higher $\beta$-estradiol and 19-norandrostenedione levels were related to more cumulus expansion $22 \mathrm{~h}$ after start of IVM ( $\beta=7.32 \% / \mathrm{ng} / \mathrm{ml}, \mathrm{p}=0.01$ and $\beta=8.55 \% / \mathrm{ng} / \mathrm{ml}, \mathrm{p}<0.01$, respectively). Follicular fluid IGF1 levels were also higher in FF compared to RES sows (Fig. 6.7A). Plasma and follicular fluid IGF1 levels $48 \mathrm{~h}$ after weaning were highly correlated (Fig. 6.7B). Higher follicular fluid IGF1 content was further related to a larger average follicle size (Fig. 6.7C) and enlarged COC expansion (Fig. 6.7D).

Relations between lactational metabolic state of the sows and follicle and oocyte developmental competence

Relations between the lactational metabolic state of the sows and follicular development $48 \mathrm{~h}$ after weaning were analysed, to get a better understanding of the underlying mechanism of metabolic influences on reproductive outcome. Only in FF sows, more lactational muscle depth loss and backfat depth loss were related to a lower average follicle size among the selected 15 largest follicles (Fig. 6.8A and Fig. 6.8B, respectively).

For both FF and RES sows, higher creatinine levels were related to a lower average follicle size (Fig. 6.8C), while for FF sows only, higher plasma leptin levels at weaning were related to a higher average follicle size (Fig. 6.8D). For both FF and RES sows, higher plasma IGF1 levels at weaning were positively related to average follicle size and percentage healthy $\operatorname{COCs}(\beta=0.007$ $\mathrm{mm} / \mathrm{ng} / \mathrm{ml}, \mathrm{p}<0.01$ and $0.12 \% / \mathrm{ng} / \mathrm{ml}, \mathrm{p}=0.05$, respectively). Finally, relations between follicular and oocyte characteristics were estimated (Supplemental Table S6.1). 
A higher average follicle size in the selected pool of 15 largest follicles was related to higher COC expansion rate 22h after IVM. A larger percentage healthy COCs increased COC expansion rate and fertilization rate, as measured $24 \mathrm{~h}$ after IVF. Increased COC expansion was related to a higher fertilization rate.

A

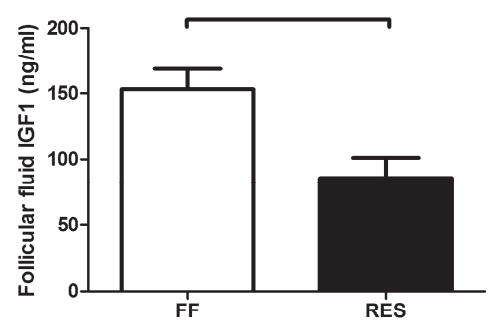

C

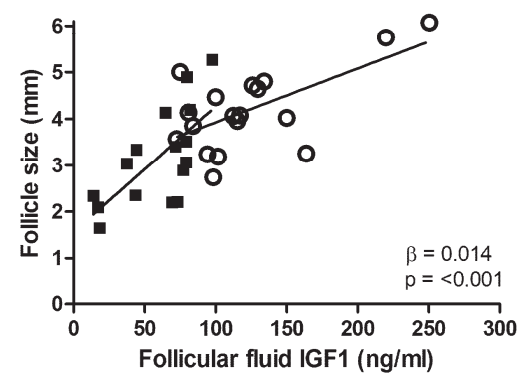

B

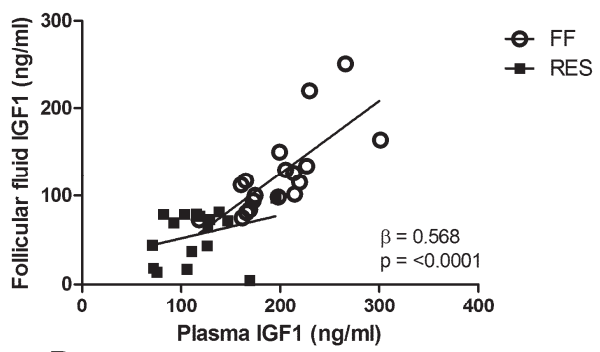

D

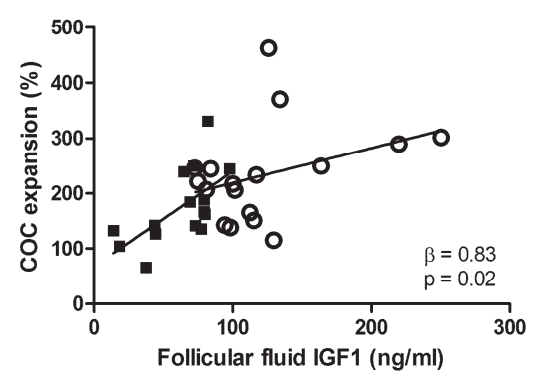

Figure 6.7 - (A) Follicular fluid levels of insulin-like growth factor 1 (IGF1) of full-fed (FF) and restrictedfed (RES) sows which received either $6.5 \mathrm{~kg} /$ day or $3.25 \mathrm{~kg} /$ day for the last two weeks of lactation and relation between follicular fluid IGF1 levels $(\mathrm{ng} / \mathrm{ml}$ ) and (B) plasma IGF1 levels at slaughter (48h after weaning) (C) average follicle size of the 15 largest follicles of both the left and right ovary or (D) COC expansion (\%) $22 \mathrm{~h}$ after start of IVM of the 15 largest follicles of the left ovary. Ovaries were obtained $48 \mathrm{~h}$ after weaning. Relations were corrected for treatment. Interactions with treatment were never significant. * P-value $<0.05$. 

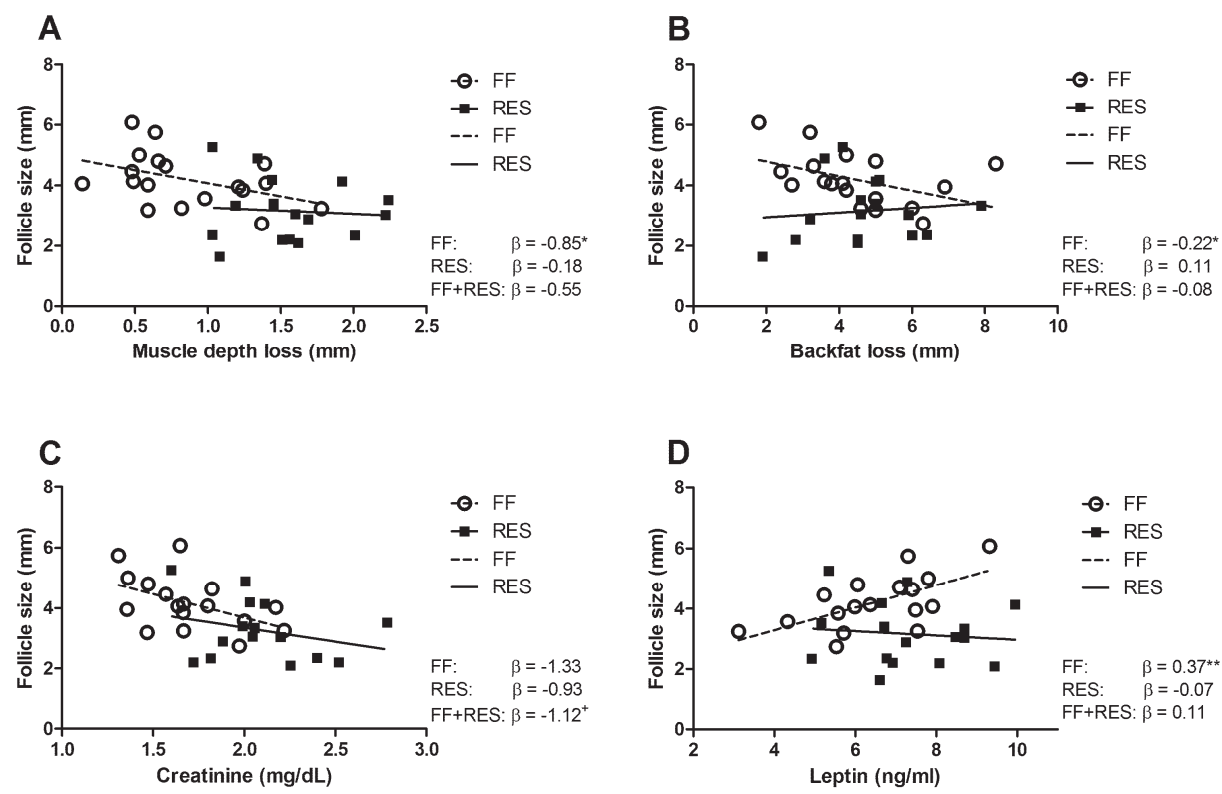

Figure 6.8 - Relations between average follicle size $(\mathrm{mm})$ of the 15 largest follicles of both left and right ovary as measured $48 \mathrm{~h}$ after weaning and $(\mathbf{A})$ muscle depth loss $(\mathrm{mm})$ during lactation (B) backfat depth loss $(\mathrm{mm})$ during lactation $(C)$ plasma creatinine $(\mathrm{mg} / \mathrm{dL})$ at weaning and (D) plasma leptin $(\mathrm{ng} / \mathrm{ml})$ at weaning. Relations were corrected for treatment. ${ }^{* *}$ P-value $<0.01,{ }^{*}$ P-value $<0.05,+$ Pvalue $\geq 0.05$ and $<0.10$.

\section{Discussion}

Sows subjected to feed restriction during the last two weeks of a 24-day lactation period lost more body weight as compared to sows which were full-fed during the entire lactation period, which as expected, resulted in lower plasma IGF1 levels at weaning. Feed restriction probably also resulted in reduced milk or low-quality milk production, as litter growth was lower in the restricted sows compared to the full-fed sows. During lactation, sows usually mobilize both lean mass and fat mass (Clowes et al., 2003; McNamara and Pettigrew, 2002). However, the difference in body weight loss in the current study mainly consisted of lean mass loss, as the restricted sows lost more loin muscle depth and had higher creatinine levels, while the loss of backfat during lactation was similar to those of the full-fed sows. Also, no differences were seen for plasma levels of leptin, a marker for adiposity, at any of the measured time-points supporting indicating no effect of feed restriction on fat mass. Lactational feed restriction usually results in higher backfat loss (Kauffold et al., 2008; Mao et al., 1999; Vinsky et al., 2006), although Patterson et al. (2011) found similar backfat loss in restricted and full-fed sows. These discrepancies might be related to the severity and duration of the feed restriction, but also to protein content of the diet and backfat thickness at the start of lactation (Costermans et al., 2019). In our study, we used TN70 gilts which have a high genetic merit for leanness (personal communication, Louisa Zak, Topigs Norsvin, 2019). Possibly, TN70 sows, 
when subjected to lactational feed restriction, rapidly mobilize more protein after onset of feed restriction as fat reserves soon become critically depleted. This might explain the lack of differences in lactational backfat loss between the restricted and full-fed sows in the current study.

The main findings of this study are that feed restriction of primiparous sows during the last two weeks of lactation negatively impacts follicle size, oocyte developmental competence in vitro and follicular fluid steroid content $48 \mathrm{~h}$ after weaning. The more severe NEB of the feed restricted sows resulted in a smaller average follicle size of the 15 largest follicles. This reduction in follicular growth could be due to an inhibition in LH pulsatility which is usually observed in sows as a response to lactational feed restriction (Kauffold et al., 2008), as metabolic intermediates influence the neuroendocrine axis in pigs (Barb et al., 2002). Subsequently, we investigated if these smaller follicles yielded COCs with a lower developmental competence. One of the ways we assessed this, was via morphological classification of COCs as either healthy (intact oocyte and cumulus cell layer) or unhealthy (damaged oocyte or cumulus cell layer), where COCs with intact oocytes and the most cumulus cell layers usually result in the highest maturation and fertilization rates (Alvarez et al., 2009; Yuan et al., 2005). When comparing the two groups of sows in our study, we found that $\mathrm{COC}$ morphology was similar between the full-fed and restricted sows. However, the more severe lactational NEB of the restricted sows did result in reduced COC expansion and a tendency in reduced fertilization rate as compared to the more moderate NEB of the full-fed sows. So, although we did not observe differences in COC morphology, the COCs of the restricted sows did show compromised developmental competence. This confirms earlier findings that a more severe lactational NEB negatively impacts in vitro oocyte maturation. For instance, feed restriction for 19 days after oestrus in gilts (Ferguson et al., 2003) and feed restriction from day 21 to 28 in a 28-day lactation period in primiparous sows (Zak et al., 1997) both resulted in a lower level of nuclear oocyte maturation. Different from these studies, we additionally analysed the developmental stage of the zygotes $24 \mathrm{~h}$ after IVF. Zygotes of restricted sows give the impression to be less developed, as fewer zygotes reached the metaphase stage at $24 \mathrm{~h}$ after IVF. In addition, a higher percentage of zygotes of restricted sows showed polyspermy. Polyspermy is a common feature of porcine IVF and is considered a marker for oocyte quality, as a higher incidence of polyspermy is seen in immature oocytes, which have not yet completed cytoplasmic maturation (Van der Ven et al., 1985) and in aged oocytes (Dalo et al., 2004). Our findings therefore indicate that zygotes of restricted sows have a reduced chance of becoming viable offspring.

Oocyte developmental competence is highly influenced by follicular fluid composition as this determines the microenvironment in which the oocytes develop. To our knowledge, this is the first study to report complete steroid profiles of full-fed and lactational feed restricted sows. Follicular fluid steroid profiling showed that $\beta$-estradiol and pregnenolone levels were lower 
for restricted sows as compared to full-fed sows. In addition, progesterone and androgen intermediates of the $\Delta 4$-pathway of steroid production (progesterone, $17 \alpha-\mathrm{OH}$-progesterone, 4-andro-3,17-stenedione and 19-norandrostenedione) were detected at lower levels in restricted sows as compared to full-fed sows. Intermediates of the $\Delta 5$-pathway of steroid production (17 $\alpha-\mathrm{OH}$-pregnenolone, DHEA and androstenediol) were below the detection limit. In human gonads, the $\Delta 5$-pathway is the preferred pathway for steroid production (reviewed by Miller and Auchus, 2011), which is reflected by the higher efficiency of CYP17A1, involved in the $\Delta 5$-pathway as compared to HSD3B which is needed for the conversion of $\Delta 5$ into $\Delta 4$-steroids (Auchus et al., 1998). The largely undetectable levels of $\Delta 5$-pathway intermediates might be explained by high $\Delta 5$-steroidogenic enzyme activity, which would indicate that the $\Delta 5$-pathway is also the preferred pathway for follicular steroid production in sows. This is further confirmed by a study which identified reduced DHEA levels in gilt saliva as a potential biomarker for the start of the waiting period in gilts (Goudet et al., 2019). The lower steroid levels in the follicular fluid of the restricted sows could at least partially explain the reduced developmental competence of the COCs in restricted sows. This is confirmed by the positive relations between $\beta$-estradiol and 19-norandrostenedione levels and COC expansion (\%) $22 \mathrm{~h}$ after start of IVM. Other studies have found that not only $\beta$-estradiol, but also higher progesterone and androgen concentrations can increase IVM and IVF outcomes when added to the maturation medium of bovine and porcine oocytes (Grupen et al., 2003; Matsuo et al., 2017; Yuan et al., 2016). Since we did not culture the oocytes in their own follicular fluid, the direct relations between $\beta$-estradiol and 19-norandrostenedione and COC expansion as found in our study, probably reflect the influence of the steroids on the developmental competence of the COCs before the start of the final maturation during IVM.

We also analysed follicular fluid IGF1 levels. The restricted sows of our study had lower follicular fluid IGF1 levels as compared to the full-fed sows. Many tissues in anabolic states produce IGF1, including the ovary. Follicular fluid IGF1 levels could therefore be dependent on peripheral IGF1, but also on FSH-induced granulosa cell IGF1 production (Hammond et al., 1988). In our study, plasma IGF1 levels were highly correlated to follicular fluid IGF levels, suggesting that peripheral IGF1 highly influenced follicular IGF1 levels, either by IGF1 transport from the serum to the follicular fluid, by influencing granulosa cell IGF1 production or both. Follicular IGF1 is essential for follicular development, as it can bind to IGF1 receptors on oocytes and granulosa cells and synergize with FSH to activate the phosphatidylinositol 3kinase signaling pathway (Law and Hunzicker-Dunn, 2016) to stimulate follicular growth, steroidogenesis, COC expansion and oocyte cleavage rate (Němcová et al., 2007; Singh and Armstrong, 1997; Xia et al., 1994; Zhou et al., 2013). The lower IGF1 levels in the restricted sows could have played an important role in the regulation of reduced follicular growth, steroid production and oocyte developmental competence. 
Finally, we analysed relations between the measured metabolic and follicular parameters to assess the impact of different metabolic intermediates on follicular development. The restricted sows lost more lean mass, but did not differ in the amount of backfat loss during lactation. Despite of this, we observed that feed restriction negatively affected follicular development, as seen by a reduced growth and changed follicular fluid composition, and the developmental competence of the oocytes. It could be speculated that especially lean mass loss negatively impacts follicular development. This is supported by findings of earlier studies, where primiparous sows which lost more lean mass but similar amounts of backfat, induced by different amounts of lysine in their diet, had smaller follicles as measured at weaning (Clowes et al., 2003) or at pro-oestrus (Yang et al., 2000). However, in our data, regression analysis showed that next to muscle depth loss, also backfat loss was negatively related to average follicle size, but in full-fed sows only. In addition, higher leptin levels at weaning were related to a higher average follicle size. This indicates that whole body adiposity and the loss of fat mass during lactation is related to follicular development around weaning. In addition, although the level of lactational backfat loss was similar between the two groups, we found that adipocytes of subcutaneous adipose tissue at slaughter were significantly smaller for restricted sows as compared to full-fed sows (Supplemental Fig. S6.2), indicative of higher lipid mobilization. This is similar to what is found in dairy cows experiencing a lactational NEB (De Koster et al., 2016; Kenéz et al., 2015). The effect of feed restriction on adipocyte size could differ between depots, as based on the measured leptin levels, whole-body lipid mobilisation and adiposity appeared to be similar between the two groups. The influence of lactational feed restriction on adipose tissue functioning of different depots and its relation with follicular development needs to be studied further.

To conclude, feed restriction of sows during the last two weeks of lactation negatively impacted follicle size, follicular fluid steroid profile and IGF1 levels, and oocyte and zygote developmental competence. Oocytes with reduced developmental competence originated from follicles with relatively low follicular fluid steroid and IGF1 concentrations. This indicates that the follicles of the restricted sows were less able to produce the necessary steroids and growth factors needed for oocytes to obtain full developmental competence. Finally, results of our study imply that the mobilization of lean mass affects follicular development more than mobilization of fat mass upon feed restriction during lactation, although a reduced fat mobilization positively influenced follicle size in full-fed sows. This knowledge improves our understanding of the relation between energy mobilisation of lean mass vs. fat mass in relation to follicle and oocyte developmental competence. Furthermore, this knowledge could eventually be used for optimal feeding in lactating sows to facilitate a better reproductive outcome. 


\section{Acknowledgements}

We acknowledge the Wageningen Institute for Animal Sciences (WIAS) and the Netherlands Organisation for Scientific Research (NWO) for providing the NWO-WIAS Graduate Programme 2015 grant. We are grateful to Manon van Marwijk, Vera Klootwijk, Pachara Paerodwong, Supawat Supanyarak and Maud Valkenaars for helping with the measurements and execution of the experiment. We also would like to thank Arjan van Dolderen and Rinie Ernste for their care for the animals.

\section{References}

Alvarez, G. M., Dalvit, G. C., Achi, M. V., Miguez, M. S., \& Cetica, P. D. (2009). Immature oocyte quality and maturational competence of porcine cumulus-oocyte complexes subpopulations. Biocell, 33(3), 167-177.

Auchus, R. J., Lee, T. C., \& Miller, W. L. (1998). Cytochrome b 5 augments the 17, 20-lyase activity of human P450c17 without direct electron transfer. Journal of biological chemistry, 273(6), 31583165.

Barb, C., Kraeling, R., \& Rampacek, G. (2002). Metabolic regulation of the neuroendocrine axis in pigs. Reproduction. Supplement, 59, 203-217.

Bergsma, R., Kanis, E., Verstegen, M., van der Peet-Schwering, C., \& Knol, E. (2009). Lactation efficiency as a result of body composition dynamics and feed intake in sows. Livestock science, 125(2), 208-222.

Blokland, M., van Tricht, E., van Ginkel, L., \& Sterk, S. (2017). Applicability of an innovative steroidprofiling method to determine synthetic growth promoter abuse in cattle. The journal of steroid biochemistry and molecular biology, 174, 265-275.

Clowes, E., Aherne, F., Foxcroft, G., \& Baracos, V. (2003). Selective protein loss in lactating sows is associated with reduced litter growth and ovarian function. Journal of animal science, 81(3), 753-764.

Costermans, N. G. J., Teerds, K. J., Keijer, J., Knol, E. F., Koopmanschap, R. E., Kemp, B., \& Soede, N. M. (2019). Follicular development of sows at weaning in relation to estimated breeding value for within-litter variation in piglet birth weight. Animal, 13(3), 554-563.

da Silva, C. L. A., Laurenssen, B., Knol, E., Kemp, B., \& Soede, N. (2016). Relationships between ovulation rate and embryonic and placental characteristics in multiparous sows at 35 days of pregnancy. Animal, 10(7), 1192-1199.

Dalo, D. T., McCaffery, J. M., \& Evans, J. P. (2004). Ultrastructural analysis of egg membrane abnormalities in post-ovulatory aged eggs. International journal of developmental biology, 52(5-6), 535-544.

De Koster, J., Van Den Broeck, W., Hulpio, L., Claeys, E., Van Eetvelde, M., Hermans, K., Hostens, M., Fievez, V., \& Opsomer, G. (2016). Influence of adipocyte size and adipose depot on the in vitro lipolytic activity and insulin sensitivity of adipose tissue in dairy cows at the end of the dry period. Journal of dairy science, 99(3), 2319-2328.

Ferguson, E., Ashworth, C., Edwards, S., Hawkins, N., Hepburn, N., \& Hunter, M. (2003). Effect of different nutritional regimens before ovulation on plasma concentrations of metabolic and reproductive hormones and oocyte maturation in gilts. Reproduction, 126(1), 61-71.

Goudet, G., Liere, P., Pianos, A., Fernandez, N., Cambourg, A., Savoie, J., Staub, C., Venturi, E., Douet, C., \& Ferchaud, S. (2019). Evolution of steroid concentrations in saliva from immature to pubertal gilts for the identification of biomarkers of gilts receptivity to boar effect. Livestock science, 228, 5-17.

Grupen, C. G., Mcllfatrick, S. M., Ashman, R. J., Boquest, A. C., Armstrong, D. T., \& Nottle, M. B. (2003). Relationship between donor animal age, follicular fluid steroid content and oocyte developmental competence in the pig. Reproduction, fertility and development, 15(2), 81-87. 
Hammond, J. M., Hsu, C.-J., Klindt, J., Tsang, B. K., \& Downey, B. R. (1988). Gonadotropins increase concentrations of immunoreactive insulin-like growth factor-l in porcine follicular fluid in vivo. Biology of reproduction, 38(2), 304-308.

Hoving, L., Soede, N., Feitsma, H., \& Kemp, B. (2012). Lactation weight loss in primiparous sows: consequences for embryo survival and progesterone and relations with metabolic profiles. Reproduction in domestic animals, 47(6), 1009-1016.

Hoving, L., Soede, N., Van der Peet-Schwering, C., Graat, E., Feitsma, H., \& Kemp, B. (2011). An increased feed intake during early pregnancy improves sow body weight recovery and increases litter size in young sows. Journal of animal science, 89(11), 3542-3550.

Kauffold, J., Gottschalk, J., Schneider, F., Beynon, N., \& Wähner, M. (2008). Effects of feeding level during lactation on FSH and LH secretion patterns, and follicular development in primiparous sows. Reproduction in domestic animals, 43(2), 234-238.

Kenéz, Á., Kulcsár, A., Kluge, F., Benbelkacem, I., Hansen, K., Locher, L., Meyer, U., Rehage, J., Dänicke, S., \& Huber, K. (2015). Changes of adipose tissue morphology and composition during late pregnancy and early lactation in dairy cows. PLOS ONE, 10(5), e0127208.

Law, N. C., \& Hunzicker-Dunn, M. E. (2016). Insulin receptor substrate 1, the hub linking folliclestimulating hormone to phosphatidylinositol 3-kinase activation. Journal of biological chemistry, 291(9), 4547-4560.

Mao, J., Zak, L., Cosgrove, J., Shostak, S., \& Foxcroft, G. (1999). Reproductive, metabolic, and endocrine responses to feed restriction and $\mathrm{GnRH}$ treatment in primiparous, lactating sows. Journal of animal science, $77(3), 724-735$.

Matsuo, M., Sumitomo, K., Ogino, C., Gunji, Y., Nishimura, R., \& Hishinuma, M. (2017). Three-step in vitro maturation culture of bovine oocytes imitating temporal changes of estradiol-17 $\beta$ and progesterone concentrations in preovulatory follicular fluid. Archives animal breeding, 60(4), 385-390.

McNamara, J., \& Pettigrew, J. (2002). Protein and fat utilization in lactating sows: I. Effects on milk production and body composition. Journal of animal science, 80(9), 2442-2451.

Miller, W. L., \& Auchus, R. J. (2011). The molecular biology, biochemistry, and physiology of human steroidogenesis and its disorders. Endocrine reviews, 32(1), 81-151.

Němcová, L., Nagyová, E., Petlach, M., Tománek, M., \& Procházka, R. (2007). Molecular mechanisms of insulin-like growth factor 1 promoted synthesis and retention of hyaluronic acid in porcine oocyte-cumulus complexes. Biology of reproduction, 76(6), 1016-1024.

Patterson, J., Smit, M., Novak, S., Wellen, A., \& Foxcroft, G. (2011). Restricted feed intake in lactating primiparous sows. I. Effects on sow metabolic state and subsequent reproductive performance. Reproduction, fertility and development, 23(7), 889-898.

Petters, R., \& Wells, K. (1993). Culture of pig embryos. Journal of reproduction and fertility. Supplement 48, 61-73.

Prunier, A., \& Quesnel, H. (2000). Influence of the nutritional status on ovarian development in female pigs. Animal reproduction science, 2(60-61), 185-197.

Quesnel, H., Pasquier, A., Mounier, A., \& Prunier, A. (1998). Influence of feed restriction during lactation on gonadotropic hormones and ovarian development in primiparous sows. Journal of animal science, 76(3), 856-863.

Schenkel, A., Bernardi, M., Bortolozzo, F., \& Wentz, I. (2010). Body reserve mobilization during lactation in first parity sows and its effect on second litter size. Livestock science, 132(1-3), 165172.

Singh, B., \& Armstrong, D. T. (1997). Insulin-like growth factor-1, a component of serum that enables porcine cumulus cells to expand in response to follicle-stimulating hormone in vitro. Biology of reproduction, 56(6), 1370-1375.

Strathe, A., Bruun, T., \& Hansen, C. (2017). Sows with high milk production had both a high feed intake and high body mobilization. Animal, 11(11), 1913-1921.

Tantasuparuk, W., Dalin, A.-M., Lundeheim, N., Kunavongkrit, A., \& Einarsson, S. (2001). Body weight loss during lactation and its influence on weaning-to-service interval and ovulation rate in 
Landrace and Yorkshire sows in the tropical environment of Thailand. Animal reproduction science, 65(3-4), 273-281.

Thaker, M. Y. C., \& Bilkei, G. (2005). Lactation weight loss influences subsequent reproductive performance of sows. Animal reproduction science, 88(3-4), 309-318.

Van der Ven, H. H., Al-Hasani, S., Diedrich, K., Hamerich, U., Lehmann, F., \& Krebs, D. (1985). Polyspermy in in vitro fertilization of human oocytes: frequency and possible causes. Annals of the new york academy of sciences, 442(1), 88-95.

Vinsky, M., Novak, S., Dixon, W., Dyck, M., \& Foxcroft, G. (2006). Nutritional restriction in lactating primiparous sows selectively affects female embryo survival and overall litter development. Reproduction, fertility and development, 18(3), 347-355.

Wientjes, J., Soede, N., Knol, E., Van den Brand, H., \& Kemp, B. (2013). Piglet birth weight and litter uniformity: Effects of weaning-to-pregnancy interval and body condition changes in sows of different parities and crossbred lines. Journal of animal science, 91(5), 2099-2107.

Wossidlo, M., Nakamura, T., Lepikhov, K., Marques, C. J., Zakhartchenko, V., Boiani, M., Arand, J., Nakano, T., Wolf, R., \& Walter, J. (2011). 5-Hydroxymethylcytosine in the mammalian zygote is linked with epigenetic reprogramming. Nature communications, 2, 241.

Xia, P., Tekpetey, F. R., \& Armstrong, D. T. (1994). Effect of IGF-I on pig oocyte maturation, fertilization, and early embryonic development in vitro, and on granulosa and cumulus cell biosynthetic activity. Molecular reproduction and development, 38(4), 373-379.

Yang, H., Foxcroft, G., Pettigrew, J., Johnston, L., Shurson, G., Costa, A., \& Zak, L. (2000). Impact of dietary lysine intake during lactation on follicular development and oocyte maturation after weaning in primiparous sows. Journal of animal science, 78(4), 993-1000.

Yuan, B., Liang, S., Jin, Y.-X., Kwon, J.-W., Zhang, J.-B., \& Kim, N.-H. (2016). Progesterone influences cytoplasmic maturation in porcine oocytes developing in vitro. PeerJ, 4, e2454.

Yuan, Y., Van Soom, A., Leroy, J., Dewulf, J., Van Zeveren, A., de Kruif, A., \& Peelman, L. (2005). Apoptosis in cumulus cells, but not in oocytes, may influence bovine embryonic developmental competence. Theriogenology, 63(8), 2147-2163.

Zak, L., Xu, X., Hardin, R., \& Foxcroft, G. (1997). Impact of different patterns of feed intake during lactation in the primiparous sow on follicular development and oocyte maturation. Journal of reproduction and fertility, 110(1), 99-106.

Zhou, P., Baumgarten, S. C., Wu, Y., Bennett, J., Winston, N., Hirshfeld-Cytron, J., \& Stocco, C. (2013). IGF-I signaling is essential for FSH stimulation of AKT and steroidogenic genes in granulosa cells. Molecular endocrinology, 27(3), 511-523. 


\section{Supplemental Material}

Supplemental Table S6.1 - Regression equations $(\beta)$ for the relations between the follicular and oocyte parameters $48 \mathrm{~h}$ after weaning; average follicle size of the 15 largest follicles, morphological classification of COC health (\%), COC expansion after $22 \mathrm{~h} \mathrm{IVM} \mathrm{( \% ),} \mathrm{fertilization} \mathrm{rate} \mathrm{( \% )} \mathrm{and} \mathrm{polyspermy}$ of fertilized oocytes (\%).

\begin{tabular}{|c|c|c|c|c|c|c|}
\hline & Treatment & $\begin{array}{l}\text { Follicle size } \\
(\mathrm{mm})\end{array}$ & $\begin{array}{l}\text { COC health } \\
\text { (\%) }\end{array}$ & $\begin{array}{c}\text { COC expansion } \\
(\%)\end{array}$ & $\begin{array}{c}\text { Fertilization } \\
\text { rate (\%) }\end{array}$ & $\begin{array}{c}\text { Polyspermy } \\
\text { (\%) }\end{array}$ \\
\hline \multirow[t]{3}{*}{ Follicle size (mm) } & FF+RES & - & -4.12 & $41.82^{* *}$ & 1.59 & 3.45 \\
\hline & FF & - & -4.29 & $46.65^{+}$ & -0.42 & 5.53 \\
\hline & RES & - & -3.93 & $37.67 *$ & 3.33 & 1.02 \\
\hline \multirow[t]{3}{*}{ COC health (\%) } & FF+RES & -0.01 & - & $1.61 *$ & $0.69 * *$ & 0.36 \\
\hline & $\mathrm{FF}$ & -0.01 & - & $1.97 *$ & 0.52 & 0.29 \\
\hline & RES & -0.01 & - & 0.95 & $1.02 * *$ & 0.42 \\
\hline \multirow[t]{3}{*}{ COC expansion (\%) } & FF+RES & $0.006^{* *}$ & $0.096 *$ & - & $0.156^{* *}$ & 0.034 \\
\hline & $\mathrm{FF}$ & $0.004^{+}$ & $0.108^{+}$ & - & $0.132^{+}$ & $0.131^{+}$ \\
\hline & RES & $0.009 *$ & 0.072 & - & $0.171^{+}$ & -0.163 \\
\hline \multirow[t]{3}{*}{ Fertilization rate (\%) } & FF+RES & 0.003 & $0.385 * *$ & $1.282 *$ & - & 0.167 \\
\hline & FF & 0.002 & $0.320^{+}$ & $1.428^{+}$ & - & 0.206 \\
\hline & RES & 0.007 & $0.523 * *$ & $1.150^{+}$ & - & 0.068 \\
\hline \multirow[t]{3}{*}{ Polyspermy (\%) } & FF+RES & 0.002 & 0.135 & 0.209 & 0.126 & - \\
\hline & FF & 0.006 & 0.220 & $1.449^{+}$ & 0.241 & - \\
\hline & RES & 0.003 & 0.145 & -0.623 & 0.049 & - \\
\hline
\end{tabular}

$\mathrm{FF}=$ full-fed, $6.5 \mathrm{~kg} /$ day for the last two weeks of lactation, RES = restricted feeding, $3.25 \mathrm{~kg} /$ day for the last two weeks of lactation. Relations were corrected for treatment. ${ }^{* *}$ P-value $<0.01^{*}, \mathrm{P}$-value $<0.05,+\mathrm{P}$-value $\geq 0.05$ and $<0.10$. 


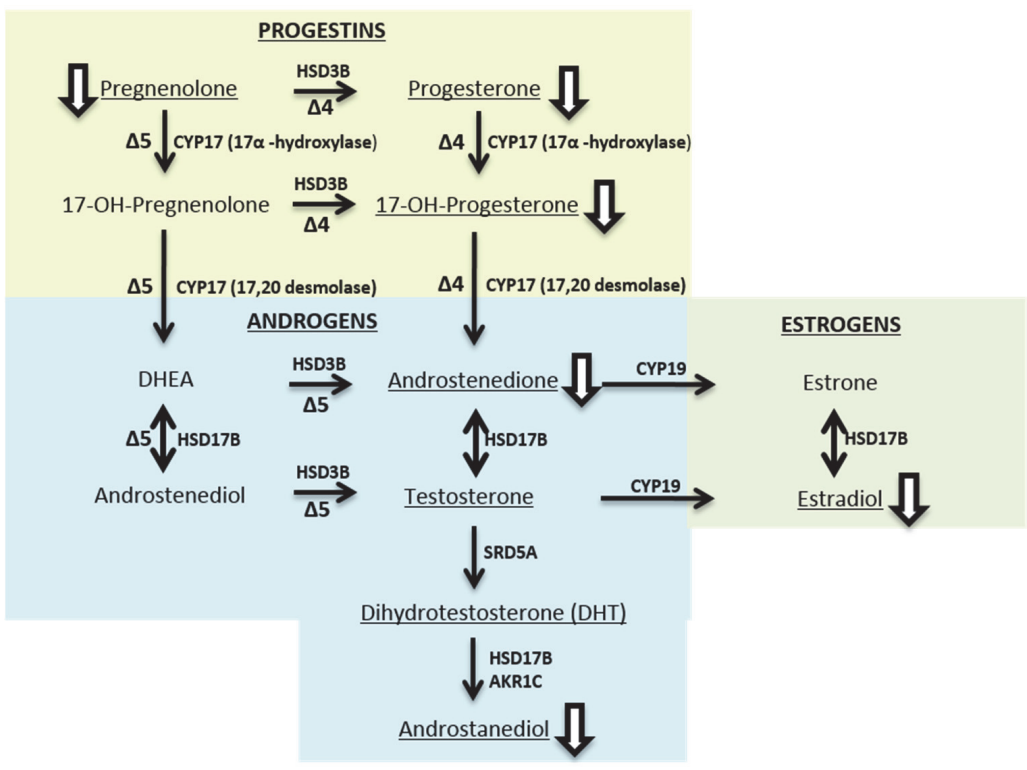

Supplemental Figure S6.1 - The effect of feed restriction of sows during the last two weeks of lactation on the follicular fluid steroid profile. The scheme depicts the steroidogenic pathway for the synthesis of progestins, androgens and estrogens (adapted from Boron, W.F. and Boulpaep, E. L., Medical Physiology $3^{\text {rd }}$ edition, 2016). The effect of feed restriction on the follicular fluid steroid profile $48 \mathrm{~h}$ after weaning (restricted-fed vs. full-fed) is shown in the scheme by the white arrows. All steroids that were detectable in the follicular fluid are underlined. The delta-4 $(\Delta 4)$ and delta-5 $(\Delta 5)$ pathway are also depicted in the scheme. CYP17 = cytochrome P450 17 $\alpha$-hydroxylase/17,20-lyase, HSD3B = 3 $\beta$ Hydroxysteroid dehydrogenase, HSD17B $=17 \beta$-Hydroxysteroid dehydrogenase, $\mathrm{CYP} 19=$ aromatase, SRD5A $=5 \alpha$-reductase, $A K R 1 C=$ aldo-keto reductase $1 \mathrm{C}$.
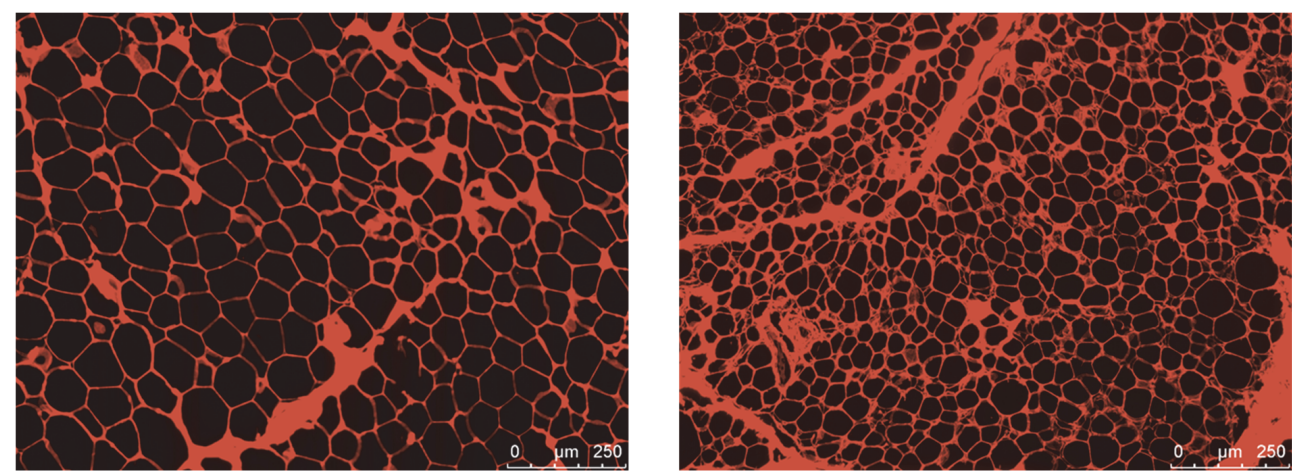

Supplemental Figure $\mathbf{S 6 . 2}$ - Representative image (100x magnification) of subcutaneous adipose tissue of full-fed (left) and restricted-fed sows which received either $6.5 \mathrm{~kg} /$ day or $3.25 \mathrm{~kg} /$ day for the last two weeks of a 24-day lactation. Adipose tissue was fixed in $4 \%$ formalin and visualised using H\&E staining and fluorescence microscopy. 


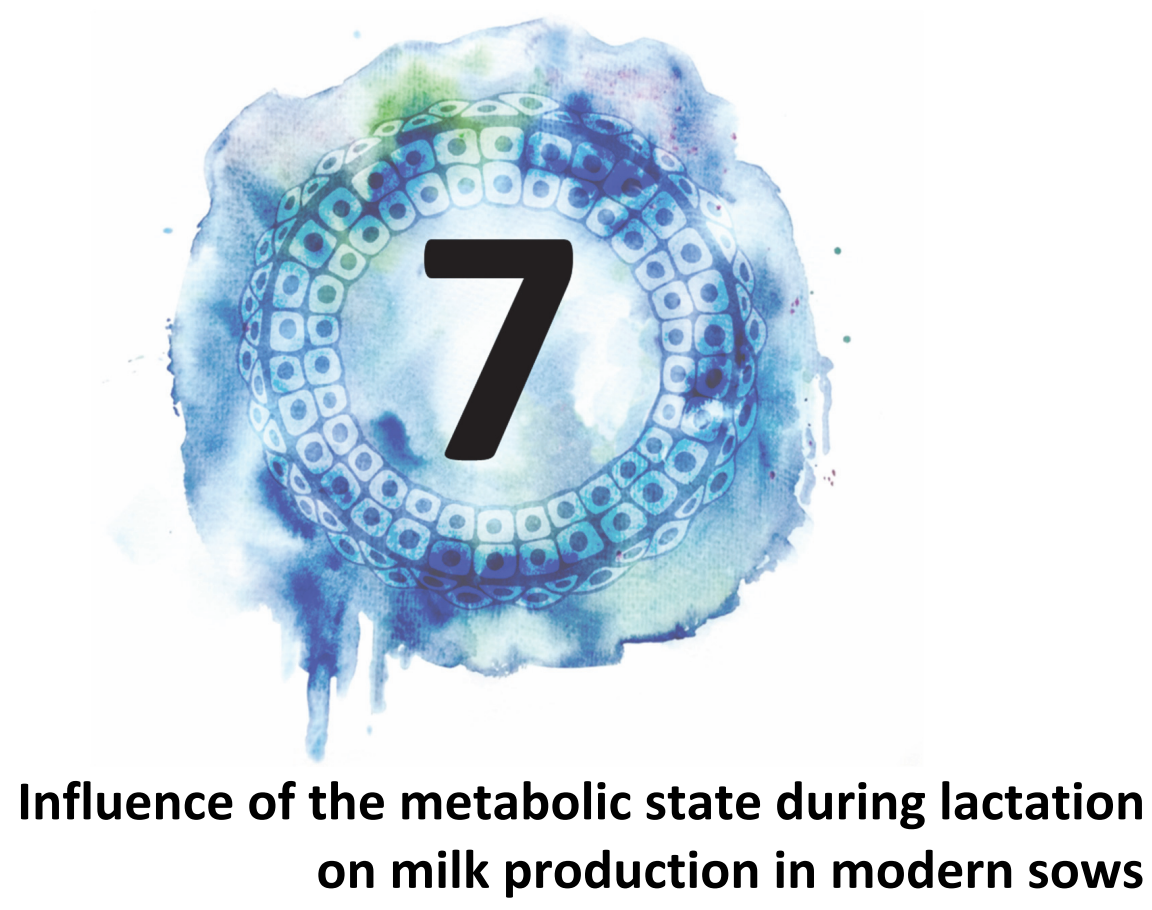

Natasja G. J. Costermans ${ }^{1,2}$, Nicoline M. Soede ${ }^{2}$, Anouschka Middelkoop ${ }^{2}$, Bjorge F. A. Laurenssen², Rudie E. Koopmanschap², Louisa Zak², Egbert F. Knol ${ }^{3}$, Jaap Keijer ${ }^{1}$, Katja J. Teerds ${ }^{1} \&$ Bas Kemp $^{2}$

${ }^{1}$ Human and Animal Physiology, Wageningen University and Research, The Netherlands ${ }^{2}$ Adaptation Physiology Group, Wageningen University and Research, The Netherlands

${ }^{3}$ Topigs Norsvin Research Center B. V., Beuningen, The Netherlands 



\begin{abstract}
Selection for prolificacy in sows has resulted in higher metabolic demands during lactation. In addition, modern sows have an increased genetic merit for leanness. Consequently, sow metabolism during lactation has changed, possibly affecting milk production and litter weight gain. The aim of this study was to investigate relations between lactational feed intake, mobilization of body tissues and milk production in modern sows. We used 36 primiparous sows which were either full-fed or restricted-fed during the last two weeks of a 24-day lactation. Restricted-fed sows had a lower milk fat percentage at weaning and lower litter weight gain in the last week of lactation. Sow body condition (body weight, loin muscle depth and backfat depth) at parturition was positively related to milk fat production in the last week of lactation. In addition, milk fat production was related to the backfat depth loss while milk protein production was related to the loin muscle depth loss during lactation. Backfat depth and loin muscle depth at parturition were positively related to lactational backfat depth loss or muscle depth loss, respectively. Together, results suggest that sows which have more resources available during lactation, either from a higher amount of body tissues at parturition, or from an increased feed intake during lactation, direct more energy towards milk production to support a higher litter weight gain. Sow management strategies to increase milk production and litter growth in modern sows may focus on improving sow body condition at the start of lactation or increasing feed intake during lactation.
\end{abstract}

Keywords: lactation, sow, metabolism, milk composition, milk production 


\section{Introduction}

Selection for high prolificacy in modern sows has led to increased litter sizes and a higher number of piglets weaned per litter (reviewed by Kemp et al., 2018). This results in higher metabolic demands during lactation due to a higher milk production. Modern sows are not able to fully compensate for these increased metabolic demands through increased feed intake (Eissen et al., 2003), especially since selection for feed efficiency has been associated with lower feed intakes (Bergsma et al., 2009; Kanis, 1990). This is especially true for primiparous sows, which on the one hand need additional protein and energy to sustain their own growth, and on the other hand have a lower feed intake capacity as compared to multiparous sows (Noblet et al., 1990; O'Grady et al., 1985). As a consequence, sows need to mobilize energy from their own body tissues during lactation, in particular from adipose tissue and skeletal muscle (Schenkel et al., 2010). As the tissue mobilization may not be sufficient to sustain optimal levels of milk production, it may reduce pre-weaning litter weight gain (De Bettio et al., 2016; Quesnel et al., 2007). This, in turn may have consequences for postweaning piglet growth and development (Cabrera et al., 2010; Wolter and Ellis, 2001; Wattanakul et al., 2007). Next to the higher metabolic demands during lactation associated with a large litter size, modern sows also have a decreased body fatness and higher capacity for gaining lean mass as compared to older lines and breeds (Ball et al., 2008). Together, these factors may have severe consequences for sow metabolism during lactation. Some studies have investigated effects of feeding different energy levels during lactation on milk production (De Bettio et al., 2016; Zhong et al., 2018) and milk composition (Noblet and Etienne, 1987; Gessner et al., 2015), with varying results. However, the consequences for energy mobilization from fat compared to skeletal muscle in relation to body reserves and feed intake has not been investigated. This is important to investigate, as in summer, sow voluntary feed intake may decrease by $50 \%$ as a result of higher ambient temperatures (Quniou and Noblet, 1999). Therefore, the aim of this study was to investigate relations between lactational feed intake, mobilization of body tissues (adipose tissue or skeletal muscle) and milk production in modern sows. This was done by subjecting primiparous sows to feed restriction during the last two weeks of lactation. Knowledge obtained by this study could help to improve sow management, e.g. breeding and feeding strategies, for optimal milk production and litter growth in modern sows.

\section{Material and methods}

The experiment was approved by the Animal Care and Use Committee of Wageningen University (DEC2017048) and performed according to national and EU guidelines at research facility CARUS of Wageningen University \& Research, the Netherlands.

\section{$\underline{\text { Animals }}$}

Experimental details were previously described by Costermans et al., (2019). The current study provides new data on milk composition and milk production estimations and describes 
relations between metabolic and milk parameters. In short, in three batches, a total of 36 TN70 (Large White x Norwegian Landrace, Topigs Norsvin, Vught, The Netherlands) gilts were used from a single gilt breeding farm. The gilts arrived on average 16 days before farrowing and received $2.9 \mathrm{~kg} /$ day of a standard gestation diet until farrowing. Piglets were cross fostered within $72 \mathrm{~h}$ after farrowing to obtain similar lactation pressure for each sow $(12.3 \pm 0.6$ (9-15) piglets per sow). During lactation, sows were fed an allocated amount of standard lactation diet (ca. 9.3 MJ NE/kg, 156 g/kg CP, 8.9 g/kg lysine, 7.69 g standard ileal digestible lysine; Maxima lacto, AgruniekRijnvallei, Wageningen, The Netherlands) divided over three meals per day. All sows received the same amount of the lactation diet until two weeks before weaning, starting at $2 \mathrm{~kg} /$ day at parturition and increasing from day 2 onwards with $0.5 \mathrm{~kg} / \mathrm{day}$ to reach $6 \mathrm{~kg} /$ day until two weeks before weaning, according to the TN70 feeding manual for primiparous sows (personal communication, Louisa Zak, Topigs Norsvin). During the last two weeks of lactation, sows were either full-fed (FF; $6.5 \mathrm{~kg} /$ day; $\mathrm{N}=18$ ) or restricted-fed (RES; 3.25 $\mathrm{kg} /$ day; $\mathrm{N}=18$ ). This resulted in an average realized feed intake of $5.9 \pm 0.1 \mathrm{vs} .3 .2 \pm 0.1 \mathrm{~kg} / \mathrm{day}$ from day 10-24, for FF and RES, respectively. The piglets were creep-fed, and litter feed intake was recorded. Sows were allocated to treatments based on body weight, backfat thickness and loin muscle depth at parturition and treatments were equally distributed over two farrowing rooms. Sows had an average lactation length of $24.1 \pm 0.3$ days for both FF and RES, and weaned $12.4 \pm 0.6$ vs. $12.3 \pm 0.6$ piglets for FF and RES, respectively.

\section{Blood and milk sampling}

Blood was sampled just before weaning (D24) as described in Costermans et al. (2019). Milk samples were obtained within $4 \mathrm{~h}$ after weaning. A single dose of $2 \mathrm{cc}$ oxytocin was given intramuscularly 20 minutes prior to collection. Milk was collected from all functional teats and pooled for each sow. Milk samples were stored at $-20^{\circ} \mathrm{C}$ until further analysis.

\section{Body weight, backfat depth, loin muscle depth}

All sows were weighed and P2 backfat depth and loin muscle depth were measured at arrival (D-16), within 24 hours after farrowing (D1), at the start of the feed restriction period (D10), at D17 and at weaning (D24). Backfat and loin muscle depth were measured in triplicate at the right and left $\mathrm{P} 2$ positions, which is located $6 \mathrm{~cm}$ from the midline, at the position of the last rib, using B-mode ultrasonography with a $6.5 \mathrm{MHz}$ linear transducer (Aquilla, Esaote, Genova, Italy), similar to Hoving et al. (2011). Piglets were weighed at birth (D0), D17 and at weaning (D24).

\section{Blood and milk measurements}

Plasma insulin, leptin, insulin-like growth factor 1 (IGF1), creatinine and serum NEFA levels were measured as described and reported in Costermans et al. (2019). Milk fat and milk protein content were analysed using the Röse-Gotlieb and Kjehldahl method (Qlip, Zutphen, The Netherlands), respectively, to obtain the percentage milk fat $(\mathrm{m} / \mathrm{m})$ and protein $(\mathrm{m} / \mathrm{m})$. Milk leptin concentrations were measured using a radioimmunoassay kit (Porcine Insulin PI- 
$12 \mathrm{~K}$ and Multi-Species Leptin XL-85K, respectively, EMD Millipore corporation, Billerica, MA, US) after centrifugation at $2150 \times g$ for 30 minutes at $4{ }^{\circ} \mathrm{C}$, similar to Pinoti and Rosi (2006).

\section{Calculations}

Milk energy content was calculated using the formula of Klaver et al. (1981): Energy $(\mathrm{kJ} / \mathrm{kg})=$ $(90.6 \mathrm{X}$ fat $(\%)+55.4 \mathrm{X}$ protein $(\%)+232.5) * 4.184$ (conversion of kcal to kJ). Average daily milk energy output of a sow in the last week of lactation was estimated using the formula of Noblet and Etienne (1989), where milk energy output $(\mathrm{kJ} / \mathrm{D})=(4.92 *$ litter gain, g/d) $(90 *$ number of piglets) $* 4.184$. Milk energy output was corrected for the feed intake of the litter (average daily feed intake between D17-22 * 9.2 MJ NE/kg). Litter gain was defined as the average daily litter weight gain in the last week of lactation (D17-24). Average milk output (kg/day) was subsequently calculated by dividing the estimated milk energy output by the calculated milk energy content at D24. Average milk protein and milk fat production ( $\mathrm{kg} /$ day) were also estimated, by multiplying the total milk production $(\mathrm{kg} / \mathrm{day})$ by the percentage milk protein or fat, respectively. Sow energy balance between D17-D24 was estimated as follows: Energy intake (average feed intake in kg/day from D17-24*9.3 MJ NE/kg) - Energy for maintenance (0.46 MJ/D x average body weight (kg) D17-24 ${ }^{0.75}$; Dourmad et al., 2008), using an efficiency factor of 0.72 for the conversion of ME to NE (NRC, 2012) - Daily milk energy output D17-24 (MJ/day). Sow nitrogen balance in the last week of lactation was estimated: Digestible protein intake (average feed intake in kg/day from D17-24*118 g/kg digestible protein) - Protein for maintenance (0.45 g protein $x$ average body weight $(\mathrm{kg})$ D17-24, according to Agricultural Research Council (ARC), 1981) - Milk output (milk protein (kg/day), and divided by 6.38 to obtain the nitrogen balance. Digestible protein for maintenance and milk output were corrected for nitrogen losses in urine by using an efficiency factor of 0.7 (ARC, 1981).

\section{Statistical analyses}

All analyses were performed using SAS 9.4 (Cary, NC, USA). Distributions of the means and residuals were examined to verify model assumptions of normality and homogeneity of variance. Milk leptin levels were log transformed to obtain normality. Parameters were considered a significant outlier when studentized residuals were $\geq 3$. A total of 7 outliers were removed from further analysis. Differences in metabolic and milk parameters between the treatment groups FF and RES were analysed using proc MIXED in SAS 9.4 in models that also contained batch as a random effect. A correction for repeated measures (day of measurement) was added to the model for analysis of body weight, backfat depth, muscle depth. Relations between metabolic parameters and milk parameters in FF and RES sows were also estimated in proc MIXED using the model: $Y_{i j}=\mu+$ treatment $_{i}+\beta i X_{i j}+\beta_{i} X_{i j}{ }^{*}$ treatment + $\varepsilon_{i j}$, where $Y_{i j}$ is the dependent variable and either a metabolic or milk parameter, $\beta$ is the regression coefficient and $\mathrm{X}_{\mathrm{ij}}$ is one of the metabolic or milk parameters. These models also 
contained batch as a random effect. Interactions were excluded from the models when not significant. All values are presented as LS means $\pm \mathrm{SE}$.

\section{Results}

\section{Effects of lactational feed restriction}

RES sows lost more body weight ( $\mathrm{kg}$ and \%) and loin muscle depth during lactation as compared to FF (see Table 7.1), but RES sows lost a similar amount of backfat depth. In the last week of lactation, however, RES sows lost more backfat depth as compared to FF sows. Percentage milk protein and milk energy content were similar between RES and FF sows, but percentage milk fat was lower in RES sows as compared to FF sows at D24. From D17-24, RES sows had a lower average daily litter gain and milk output as compared to FF sows (Table 7.1).

Table 7.1 - Body condition, milk composition and milk output of primiparous sows that were either full-fed $(6.5 \mathrm{~kg} /$ day) or restricted-fed $(3.25 \mathrm{~kg} /$ day) from D10-D24 during a 24-day lactation.

\begin{tabular}{|c|c|c|c|}
\hline & Full-fed & Restricted-fed & P-value \\
\hline \multicolumn{4}{|l|}{ Sow metabolic state } \\
\hline \multicolumn{4}{|l|}{ Day 1 - parturition } \\
\hline Body weight (kg) & $206 \pm 5$ & $204 \pm 5$ & 0.74 \\
\hline Loin muscle depth (mm) & $5.4 \pm 0.1$ & $5.6 \pm 0.1$ & 0.26 \\
\hline Backfat depth (mm) & $14.1 \pm 0.6$ & $14.2 \pm 0.6$ & 0.85 \\
\hline \multicolumn{4}{|l|}{ Day 24 - weaning } \\
\hline Body weight (kg) & $183 \pm 5$ & $164 \pm 5$ & $<0.01$ \\
\hline Loin muscle depth (mm) & $4.4 \pm 0.1$ & $4.0 \pm 0.1$ & 0.01 \\
\hline Backfat depth (mm) & $9.5 \pm 0.6$ & $9.0 \pm 0.6$ & 0.54 \\
\hline \multicolumn{4}{|l|}{ Day 1-24 } \\
\hline Body weight loss (kg) & $23.6 \pm 3.1$ & $40.1 \pm 3.1$ & $<0.001$ \\
\hline Body weight loss (\%) & $11.5 \pm 1.4$ & $19.6 \pm 1.4$ & $<0.001$ \\
\hline Loin muscle depth loss (mm) & $1.1 \pm 0.1$ & $1.7 \pm 0.1$ & $<0.001$ \\
\hline Backfat depth loss (mm) & $4.6 \pm 0.6$ & $5.1 \pm 0.6$ & 0.34 \\
\hline \multicolumn{4}{|l|}{ Day $17-24$} \\
\hline Body weight loss (kg) & $8.0 \pm 0.8$ & $12.4 \pm 0.8$ & $<0.01$ \\
\hline Body weight loss (\%) & $4.2 \pm 0.4$ & $7.0 \pm 0.4$ & $<0.001$ \\
\hline Loin muscle depth loss (mm) & $0.3 \pm 0.1$ & $0.7 \pm 0.1$ & $<0.01$ \\
\hline Backfat depth loss (mm) & $0.1 \pm 0.4$ & $0.6 \pm 0.4$ & 0.02 \\
\hline Energy balance (MJ/day) & $-21.6 \pm 2.4$ & $-32.6 \pm 2.5$ & $<0.001$ \\
\hline Nitrogen balance (g/day) & $-21.0 \pm 6.2$ & $-46.2 \pm 6.0$ & $<0.01$ \\
\hline Litter gain (kg/day) & $3.1 \pm 0.1$ & $2.5 \pm 0.1$ & $<0.001$ \\
\hline \multicolumn{4}{|l|}{ Milk parameters D24 } \\
\hline Milk protein (\%) & $5.3 \pm 0.2$ & $5.4 \pm 0.2$ & 0.49 \\
\hline Milk fat (\%) & $6.9 \pm 0.2$ & $6.3 \pm 0.2$ & 0.05 \\
\hline Milk leptin (log; ng/ml) & $1.45 \pm 0.01$ & $1.46 \pm 0.01$ & 0.72 \\
\hline Estimated energy content (MJ/kg) & $4.8 \pm 0.1$ & $4.6 \pm 0.1$ & 0.14 \\
\hline \multicolumn{4}{|l|}{ Calculated milk production D17-24 } \\
\hline Milk production (kg/day) & $13.0 \pm 0.4$ & $10.3 \pm 0.4$ & $<0.001$ \\
\hline Milk protein (g/day) & $680 \pm 25$ & $552 \pm 25$ & 0.01 \\
\hline Milk fat (g/day) & $870 \pm 33$ & $646 \pm 32$ & $<0.001$ \\
\hline
\end{tabular}


$\underline{\text { Relations between metabolic state and milk production }}$

Relations between the metabolic state of the sows at parturition and at weaning are shown in Table 7.2. Interactions with treatment were never significant. More loin muscle depth at D1 and a higher body weight at D1 were related to a higher litter weight gain, total milk production and milk fat production from D17-24. Loin muscle depth and body weight at D24 were not related to milk production parameters. More backfat depth at D1, but not backfat at D24, was related to a higher milk fat production from D17-24. Sow body weight, loin muscle depth or backfat depth were never related to milk fat content, milk protein content, energy content or leptin levels at D24.

Table 7.2 - Regression equations $(\beta)$ for the relations between body weight $(x, k g)$, loin muscle depth $(\mathrm{x}, \mathrm{mm})$, backfat depth (x, mm) at parturition (D1) and at weaning (D24) and average daily litter gain and milk output parameters from D17-24 (y). Treatment (full-fed (6.5 kg/day) or restricted-fed (3.25 $\mathrm{kg} /$ day) from D10-24 of a 24-day lactation) was included in the model. Interactions with treatment were never significant.

\section{Day 17-24}

\begin{tabular}{cccccccc}
\hline $\begin{array}{c}\text { Litter gain } \\
\text { (g/day) }\end{array}$ & \multicolumn{2}{c}{$\begin{array}{c}\text { Milk output } \\
\text { (kg/day) }\end{array}$} & \multicolumn{2}{c}{$\begin{array}{c}\text { Milk protein } \\
\text { (g/day) }\end{array}$} & \multicolumn{2}{c}{ Milk fat (g/day) } \\
\hline$\beta$ & $\mathrm{p}$ & $\beta$ & $\mathrm{p}$ & $\beta$ & $\mathrm{p}$ & $\beta$ & $\mathrm{p}$ \\
\hline $\mathbf{9 . 2 6}$ & $\mathbf{0 . 0 1}$ & $\mathbf{0 . 0 5}$ & $<0.01$ & 1.63 & 0.09 & $\mathbf{2 . 8 6}$ & $<0.01$ \\
3.67 & 0.24 & 0.03 & 0.15 & 0.30 & 0.77 & 1.48 & 0.15 \\
& & & & & & & \\
$\mathbf{2 6 3}$ & $<\mathbf{0 . 0 1}$ & $\mathbf{1 . 3 3}$ & $\mathbf{0 . 0 1}$ & 36.2 & 0.25 & $\mathbf{8 1 . 5}$ & $<0.01$ \\
96.7 & 0.35 & 0.25 & 0.65 & -16.4 & 0.61 & 50.2 & 0.14 \\
& & & & & & & \\
43.3 & 0.06 & 0.19 & 0.12 & 7.57 & 0.28 & $\mathbf{1 5 . 3}$ & $\mathbf{0 . 0 3}$ \\
10.1 & 0.75 & 0.07 & 0.66 & 2.32 & 0.82 & 9.49 & 0.37 \\
\hline
\end{tabular}

\section{Relations between changes in metabolic state and milk production}

Relations between body weight loss, loin muscle depth loss or backfat loss and milk production parameters are shown in Fig. 7.1. Interactions with treatment were never significant. Body weight loss, loin muscle depth loss and backfat depth loss from D1-24 were not related to milk fat content, milk protein content or milk energy content at D24, but were related to a higher average daily litter weight gain (D17-24). A higher body weight loss (D1-24) was also related to higher milk protein production and milk fat production (D17-24). A higher loin muscle depth loss (D1-24) was related to a higher milk production and protein production but not related to a higher milk fat production, while a higher backfat depth loss (D1-24) was related to a higher milk production and milk fat production but not related to a higher milk protein production (Fig. 7.1). A higher backfat depth loss from D17-24 and higher serum NEFA levels at D24 were related to a higher milk fat content and milk energy content at D24 (Fig. 
7.2). Plasma IGF1, insulin, creatinine and leptin levels were never related to milk composition or production parameters.
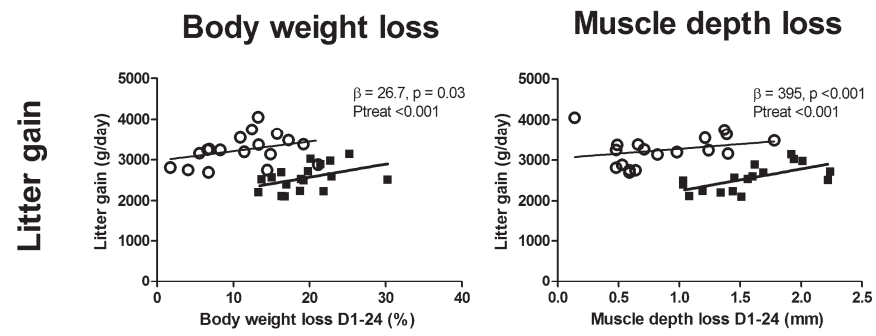

\section{Backfat depth loss}
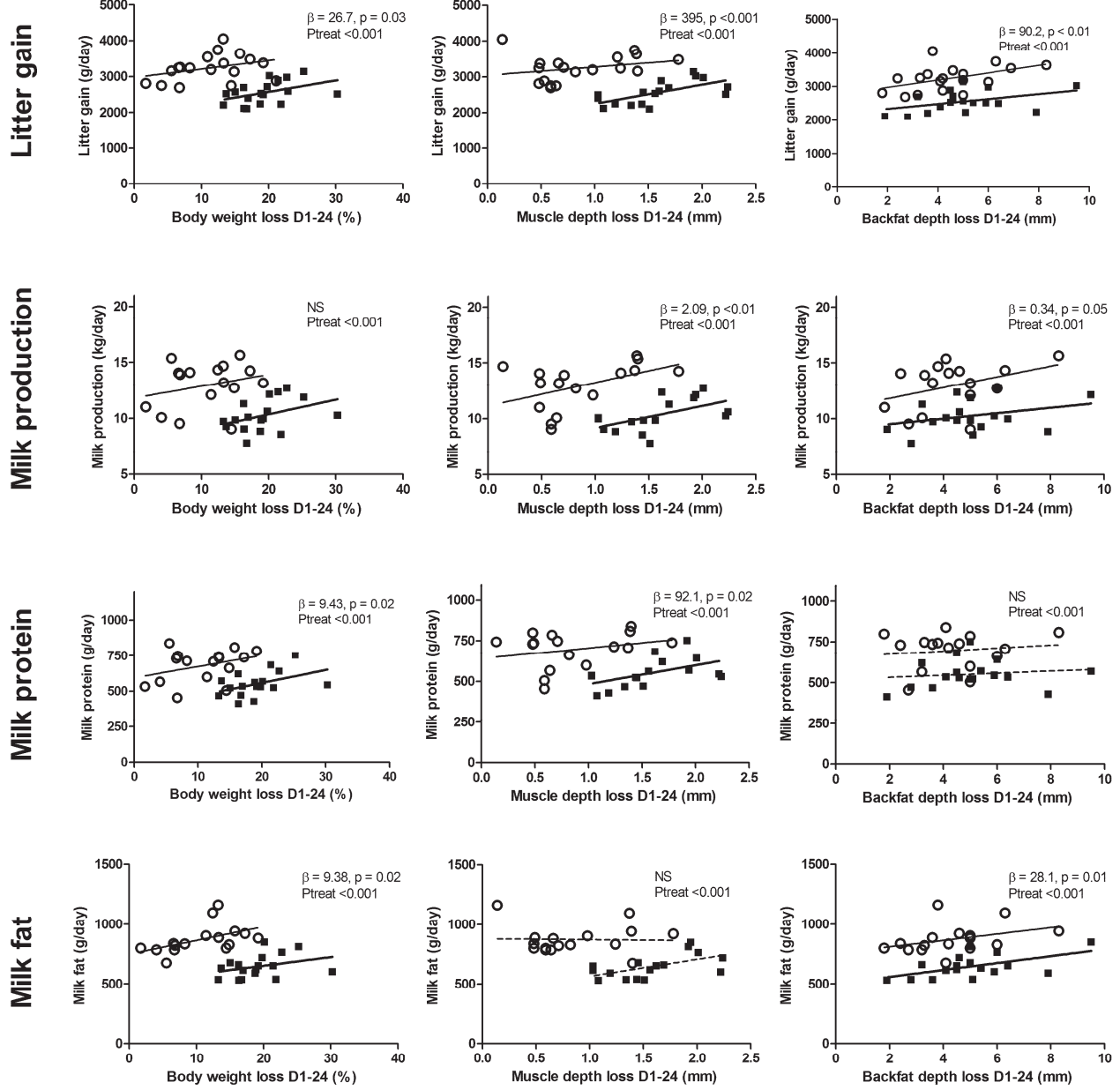

Figure 7.1 - Regression equations $(\beta)$ for the relations between body weight loss, loin muscle depth loss, backfat depth loss from D1-24 and milk production parameters. Treatment (full-fed (6.5 kg/day), open circles 0 , or restricted-fed (3.25 kg/day), filled squares $\mathbf{m}$, from D10-24 of a 24-day lactation) was included in the model. Interactions with treatment were never significant. Ptreat $=p$-value for treatment. 
Relations between energy or nitrogen balance and milk production are shown in Fig. 7.3. A more negative energy or nitrogen balance was related to higher litter gain, total milk, milk fat and milk protein production from D17-24. For FF sows only, a lower nitrogen balance was related to a higher milk energy content at $D 24(\beta=0.01, p<0.01)$. Relations between individual milk production parameters are shown in Supplemental Table S1.
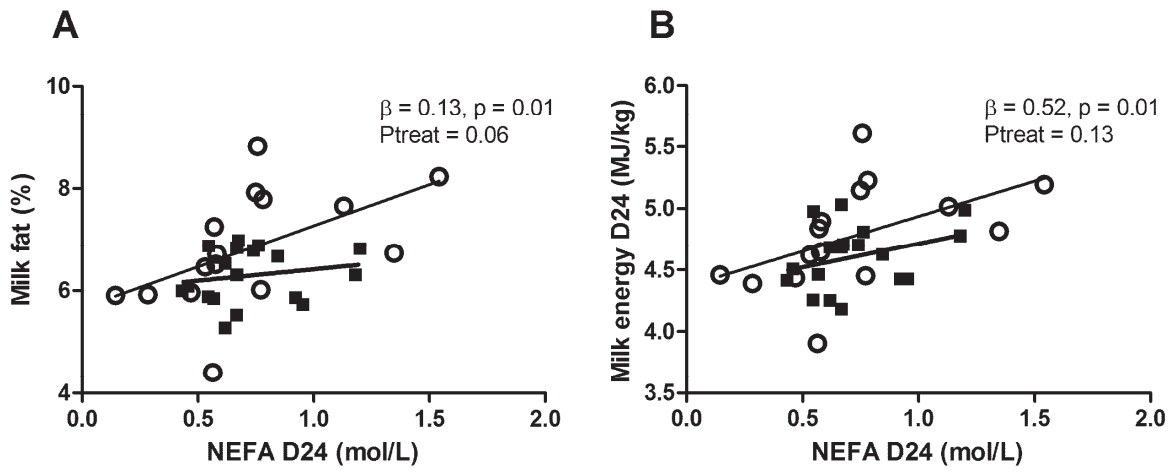

Figure 7.2 - Regression equations ( $\beta$ ) for the relations between serum NEFA levels at D24 and (A) percentage milk fat at D24 or (B) milk energy content at D24. Treatment (full-fed $(6.5 \mathrm{~kg} / \mathrm{day})$, open circles $\mathrm{O}$, or restricted-fed (3.25 kg/day), filled squares $\mathbf{m}$, from D10-24 of a 24-day lactation) was included in the model. Interactions with treatment were never significant. Ptreat $=p$-value for treatment. 

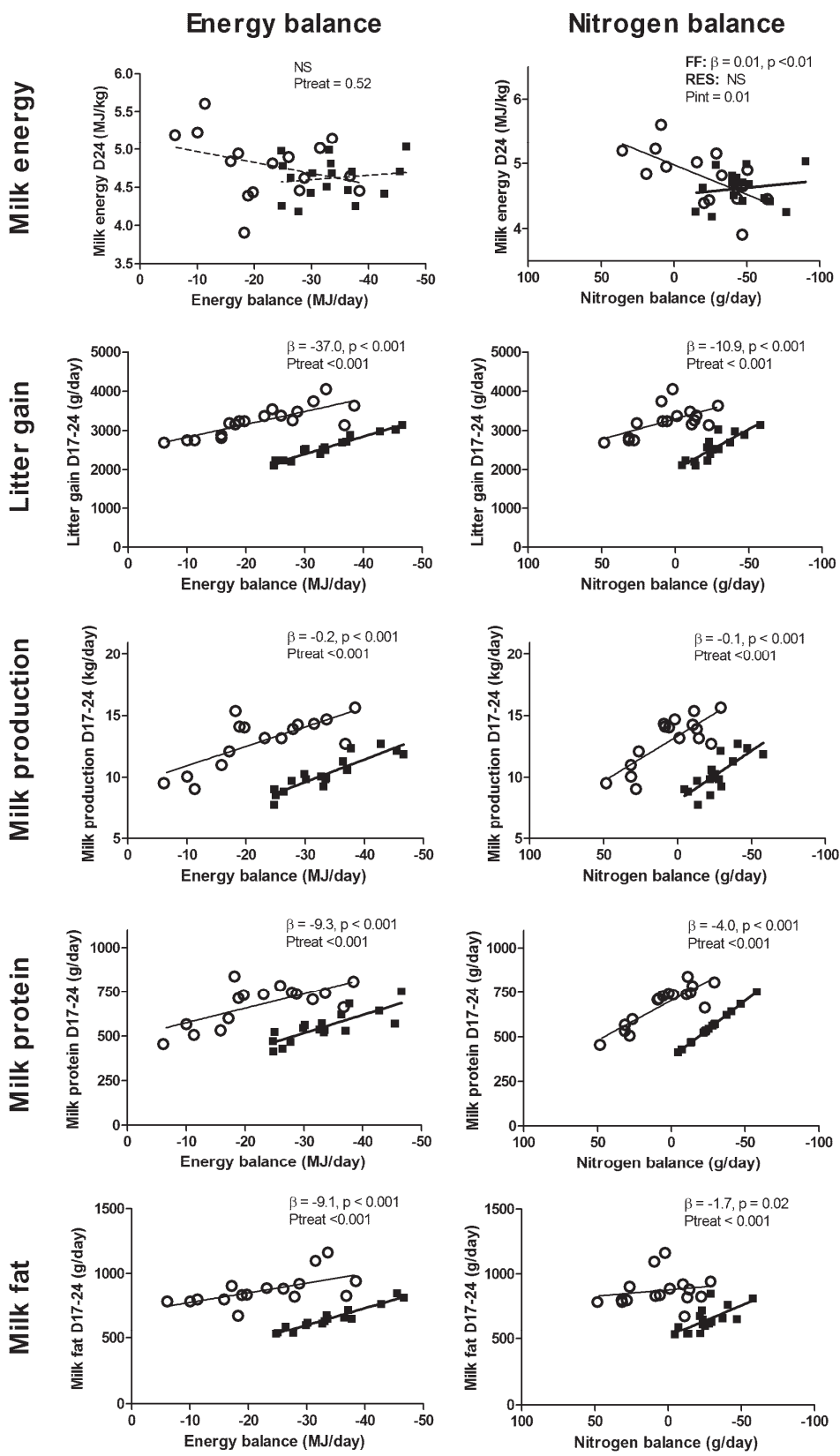

Figure 7.3 - Regression equations $(\beta)$ for the relations between estimated daily energy balance and nitrogen balance from D17-24 and milk production parameters. Treatment (full-fed (6.5 kg/day), open circles 0 , or restricted-fed (3.25 kg/day), filled squares $\mathbf{m}$, from D10-24 of a 24-day lactation) was included in the model. Interactions with treatment are indicated where significant. Pint $=p$-value for interaction and Ptreat $=p$-value for treatment. 
Relations between metabolic state at parturition and body tissue mobilization during lactation Loin muscle depth at D1 was positively related to loin muscle depth loss during lactation and backfat depth at D1 was positively related to backfat depth loss during lactation. Body weight at D1 was not related to body weight loss during lactation (Fig. 7.4).
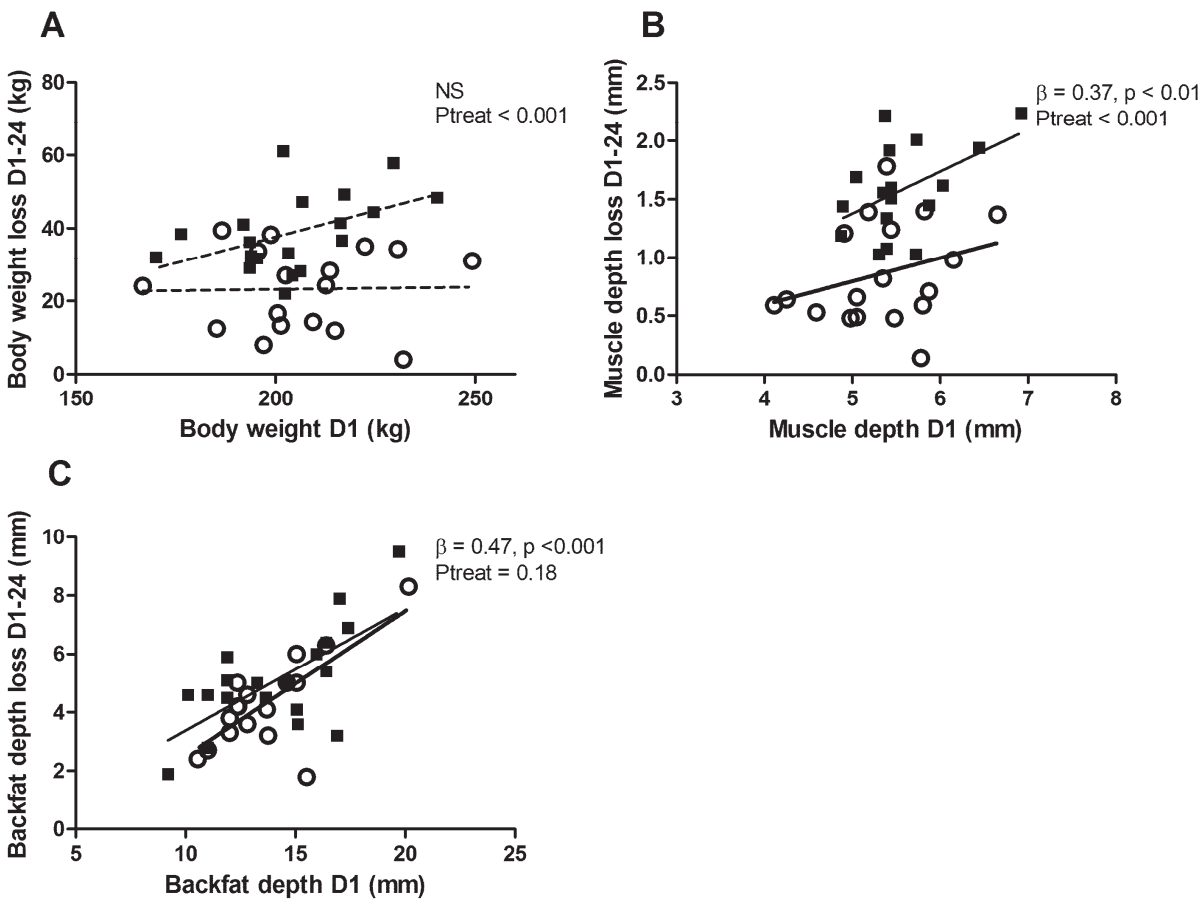

Figure 7.4 - Regression equations $(B)$ for the relations between $(A)$ body weight at parturition and weight loss during lactation, (B) loin muscle depth at parturition and loin muscle depth loss during lactation and (C) backfat depth at parturition and backfat depth loss during lactation. Treatment (fullfed $(6.5 \mathrm{~kg} /$ day), open circles 0 , or restricted-fed $(3.25 \mathrm{~kg} /$ day), filled squares $\mathbf{m}$, from D10-24 of a 24 day lactation) was included in the model. Interactions with treatment are indicated where significant. Pint $=p$-value for interaction and Ptreat $=$ P-value for treatment.

\section{Discussion}

\section{Effects of lactational feed restriction}

In the current study, primiparous sows were subjected to feed restriction during the last two weeks of lactation, to study effects of body tissue mobilization on milk production and composition. As expected, primiparous sows subjected to feed restriction mobilized more body tissues as compared to full-fed sows. Also, lactational feed restriction influenced milk composition by lowering fat content, while protein content and milk energy levels were similar. Next to effects on milk composition, lactational feed restriction also reduced total milk production, which was due to both lower milk fat and milk protein production. This is different from what has been found in previous studies. Both Gessner et al. (2015) and Noblet and Etienne (1987) applied around 25\% feed restriction during a 21-day lactation in second parity 
and primiparous sows, respectively. Gessner et al. (2015) found no effect on milk production or composition while Noblet and Etienne (1987), describe a lower milk yield with a higher fat, nitrogen and energy content. Differences in effects of feed restriction on milk production and composition may be explained by factors such as sow feed composition, the number of suckling piglets and duration of lactation. However, the most pronounced difference between the described studies was the level of feed restriction. The $50 \%$ feed restriction in the current study resulted in a relative weight loss of $19.6 \%$ vs. $11.5 \%$ in full-fed sows. Gessner et al., (2015) describe a relative weight loss of 13 vs. $7.8 \%$ in restricted-fed and full-fed, respectively, while Noblet and Etienne (1987) describe a relative weight loss of 8.2 vs. $6.4 \%$. The higher relative weight loss of the restricted-fed sows in the current study, may have reduced energy and nutrient availability to such an extent that the sows were not able to sustain high levels of milk fat production and milk protein production.

Relations between the metabolic state and milk production

A higher milk fat production in the last week of lactation was related with higher body weight, loin muscle depth and backfat depth at the start of lactation. These results may suggest that sows with more body reserves at the start of lactation, have more available energy and metabolites to mobilize, which are subsequently used to sustain a high level of milk fat production. Indeed, sows with more backfat at parturition and more muscle depth at parturition, mobilized more backfat depth and muscle depth during lactation, respectively. In addition, a higher milk fat content of the milk at weaning was related to higher NEFA levels at weaning (as a measure for adipose tissue mobilization). NEFA in the blood can be transported to mammary gland capillaries, and thereby contribute to milk fat synthesis (Boyd et al., 1995). Higher adipose tissue mobilization may therefore increase milk fat content by increasing NEFA levels in the blood, as is also found in dairy cattle (Bell, 1995; Lerch et al., 2015).

Milk protein production was not related to body condition at the start of parturition. However, higher milk protein production in the last week of lactation was related to more muscle depth loss (as a measure for skeletal muscle mobilization) during lactation. Interestingly, milk fat production was not related to muscle depth mobilization. Similarly, milk fat production was only related to backfat depth loss and not to muscle depth loss. Together, this may indicate that the type of body tissues, adipose tissue or skeletal muscle mass, that are mobilized during lactation are related to level of either milk fat or protein production, respectively. These findings are important, as milk is the main source of energy and nutrients for piglets during lactation and its composition influences pre-weaning piglet growth and body composition (Lauridsen and Danielsen, 2004; Salcedo et al., 2016; Van den Brand et al., 2000; Yang et al., 2009), which in turn, determines piglet growth after weaning (Cabrera et al., 2010; Wolter and Ellis, 2001). For instance, van den Brand et al. (2000) found that feeding a fat-rich vs. a starchrich diet at a high feeding level (44MJ NE/day for both diets) during a 21-day lactation, increased milk fat production, litter growth and piglet body fat concentration. All of these above described relations between the sows' metabolic state and milk production, were independent of feed level during lactation. 
Next to milk fat and protein content, we analysed milk leptin levels, one of the key hormones in milk. Studies in humans and rodents suggest that milk leptin levels reflect the metabolic state and feed intake of the mother (e.g. Nozhenko et al., 2015; Sadr Dadres et al., 2019; Savino et al., 2016), and in rats, leptin regulates short-term feed intake in the suckling period (Sanchéz et al., 2005) and may regulate later life (metabolic) health in offspring (reviewed by Palou et al., 2018). Milk leptin can be transferred from plasma (Casabiell et al., 1997) or produced locally by the mammary gland (Bonnet et al., 2002). In the current study, plasma leptin levels were not related to milk leptin levels $(\beta<0.001, p=0.96)$. This corroborates findings of Estienne et al., (2000), in second parity sows. This suggests that milk leptin levels largely arise from mammary gland leptin synthesis in sows. Milk leptin levels at weaning appeared not affected by feed restriction during lactation and were not related to lactational metabolic parameters. This is different from the gestation period, where different feeding levels and subsequent level of adiposity determine milk leptin levels around farrowing (Estienne et al., 2003). Furthermore, no relations between milk leptin and pre-weaning piglet feed intake and litter growth could be found. It is also unclear if milk leptin in sows, similar to what is found for rodents, plays a role in regulating piglet growth and performance.

To summarize, $50 \%$ feed restriction during the last two weeks of a 24-day lactation reduced milk fat content and milk yield in primiparous modern sows. Milk production is highly influenced by the sows' metabolic state (body weight, backfat depth and muscle depth) at the start of lactation and mobilization of body tissues during lactation. In addition, the level of milk fat and milk protein production at the end of lactation was related to the amount of mobilized adipose tissue or skeletal muscle, respectively. Together, our results suggest that sows which have more available resources during lactation, either from a higher amount of mobilisable protein or fat from body tissues at the start of lactation, or by increased feed intakes during lactation, have a higher milk production to support a higher litter weight gain. Our results also imply that it is especially challenging to optimize milk production in primiparous sows, which have less metabolisable body tissues and have a lower feed intake as compared to older sows (Noblet et al., 1990; O'Grady et al., 1985). To improve litter gain during lactation, it would be beneficial to increase the amount of body tissues at the start of lactation, either by increasing the age that gilts are bred or by optimizing feed intake during gestation. However, one difficulty of this latter strategy is that heavier and fatter sows at parturition have a reduced voluntary feed intake during lactation (reviewed by Eissen et al., 2000; Quesnel et al., 2005) which in turn, decreases available resources for milk production during lactation. It is clear that these strategies should be carefully evaluated to fully optimize sow milk production during lactation. In addition, our results show that the type of milk nutrients that sows produce, milk fat or milk protein, is highly related to the type of body tissues, adipose tissue or skeletal muscle, that are mobilized during lactation. This suggests that the amount of milk protein and milk fat production of sows can be controlled by feeding strategies. As the amount of milk protein production may be insufficient for maximum piglet gain (Campbell and Dunkin, 1983), sow feeding strategies may be directed towards a higher milk protein output to increase litter weight gain. 


\section{Acknowledgements}

We acknowledge the Wageningen Institute for Animal Sciences (WIAS) and the Netherlands Organisation for Scientific Research (NWO) for providing the NWO-WIAS Graduate Programme 2015 grant. We are grateful to Manon van Marwijk, Pachara Paerodwong, Supawat Supanyarak and Maud Valkenaars for helping with the measurements and execution of the experiment. We also would like to thank Arjan van Dolderen and Rinie Ernste for their care for the animals.

\section{References}

Agricultural Research Council (ARC). (1981). "Protein and amino acid requirements" in: The nutrient requirements of pigs: technical review by an Agricultural Research Council Working Party, second edition. CAB, Slough, UK.

Ball, R. O., Samuel, R., \& Moehn, S. (2008). Nutrient requirements of prolific sows. Advances in pork production 19, 223-236.

Bell, A. W. (1995). Regulation of organic nutrient metabolism during transition from late pregnancy to early lactation. Journal of animal science 73(9), 2804-2819.

Bergsma, R., Kanis, E., Verstegen, M., van der Peet-Schwering, C., \& Knol, E. (2009). Lactation efficiency as a result of body composition dynamics and feed intake in sows. Livestock science, 125(2), 208-222.

Bonnet, M., Delavaud, C., Laud, K., Gourdou, I., Leroux, C., Djiane, J., \& Chilliard, Y. (2002). Mammary leptin synthesis, milk leptin and their putative physiological roles. Reproduction nutrition development, 42(5), 399-413.

Boyd, D. R., Kensinger, R. S., Harrell, R. J., \& Bauman, D. E. (1995). Nutrient uptake and endocrine regulation of milk synthesis by mammary tissue of lactating sows. Journal of animal science. Supplement 73(2), 36-56.

Cabrera, R., Boyd, R., Jungst, S., Wilson, E., Johnston, M., Vignes, J., \& Odle, J. (2010). Impact of lactation length and piglet weaning weight on long-term growth and viability of progeny. Journal of animal science, 88(7), 2265-2276.

Campbell, R., \& Dunkin, A. (1983). The influence of protein nutrition in early life on growth and development of the pig: 1 . Effects on growth performance and body composition. British journal of nutrition 50(3), 605-617.

Casabiell, X., Pineiro, V., Tome, M., Peino, R., Dieguez, C., \& Casanueva, F. (1997). Presence of leptin in colostrum and/or breast milk from lactating mothers: a potential role in the regulation of neonatal food intake. The journal of clinical endocrinology \& metabolism, 82(12), 4270-4273.

Costermans, N. G. J., Teerds, K. J., Middelkoop, A., Roelen, B. A. J., Schoevers, E. J., Van Tol, H. T. A., Laurenssen, B., Koopmanschap, R. E., Zhao, Y., Blokland, M., Van Tricht, F., Zak, L., Keijer, J., Kemp, B., \& Soede, N. M. (2019). Consequences of negative energy balance on follicular development and oocyte quality in primiparous sows. Biology of reproduction. In press. DOI:10.1093/biolre/ioz175.

De Bettio, S., Maiorka, A., Barrilli, L., Bergsma, R., \& Silva, B. (2016). Impact of feed restriction on the performance of highly prolific lactating sows and its effect on the subsequent lactation. Animal, 10(3), 396-402.

Dourmad, J.-Y., Etienne, M., Valancogne, A., Dubois, S., Van Milgen, J., \& Noblet, J. (2008). InraPorc: a model and decision support tool for the nutrition of sows. Animal feed science and technology 143(1-4), 372-386.

Eissen, J., Kanis, E., \& Kemp, B. (2000). Sow factors affecting voluntary feed intake during lactation. Livestock production science 64(2-3), 147-165. 
Eissen, J., Apeldoorn, E., Kanis, E., Verstegen, M., \& De Greef, K. (2003). The importance of a high feed intake during lactation of primiparous sows nursing large litters. Journal of animal science, 81(3), 594-603.

Estienne, M., Harper, A., Barb, C., \& Azain, M. (2000). Concentrations of leptin in serum and milk collected from lactating sows differing in body condition. Domestic animal endocrinology, 19(4), 275-280.

Estienne, M., Harper, A., Kozink, D., \& Knight, J. (2003). Serum and milk concentrations of leptin in gilts fed a high-or low-energy diet during gestation. Animal reproduction science, 75(1-2), 95105.

Gessner, D. K., Gröne, B., Rosenbaum, S., Most, E., Hillen, S., Becker, S., Erhardt, G., Reiner, G., Ringseis, R., \& Eder, K. (2015). Effect of a negative energy balance induced by feed restriction in lactating sows on hepatic lipid metabolism, milk production and development of litters. Archives of animal nutrition, 69(5), 399-410.

Hoving, L., Soede, N., Van der Peet-Schwering, C., Graat, E., Feitsma, H., \& Kemp, B. (2011). An increased feed intake during early pregnancy improves sow body weight recovery and increases litter size in young sows. Journal of animal science, 89(11), 3542-3550.

Kanis, E. (1990). Effect of food intake capacity on production traits in growing pigs with restricted feeding. Animal science, 50(2), 333-341.

Kemp, B., Da Silva, C., \& Soede, N. M. (2018). Recent advances in pig reproduction: Focus on impact of genetic selection for female fertility. Reproduction in domestic animals. Supplement 53(2), 28-36.

Klaver, J., Van Kempen, G., De Lange, P., Verstegen, M., \& Boer, H. (1981). Milk composition and daily yield of different milk components as affected by sow condition and lactation/feeding regimen. Journal of animal science, 52(5), 1091-1097.

Lauridsen, C., \& Danielsen, V. (2004). Lactational dietary fat levels and sources influence milk composition and performance of sows and their progeny. Livestock production science 91(12), 95-105.

Lerch, S., Pires, J., Delavaud, C., Shingfield, K., Pomies, D., Martin, B., Chilliard, Y., \& Ferlay, A. (2015). Rapeseed or linseed in dairy cow diets over 2 consecutive lactations: Effects on adipose fatty acid profile and carry-over effects on milk fat composition in subsequent early lactation. Journal of dairy science 98(2), 1005-1018.

Noblet, J., \& Etienne, M. (1987). Metabolic utilization of energy and maintenance requirements in lactating sows. Journal of animal science, 64(3), 774-781.

Noblet, J., \& Etienne, M. (1989). Estimation of sow milk nutrient output. Journal of animal science, 67(12), 3352-3359.

Noblet, J., Dourmad, J., \& Etienne, M. (1990). Energy utilization in pregnant and lactating sows: modeling of energy requirements. Journal of animal science, 68(2), 562-572.

Nozhenko, Y., Asnani-Kishnani, M., Rodríguez, A. M., \& Palou, A. (2015). Milk leptin surge and biological rhythms of leptin and other regulatory proteins in breastmilk. PLOS ONE, 10(12), e0145376.

National Research Council (NRC). (2012). Nutrient Requirements of Swine eleventh revised edition. National Academic Press, Washington, D.C. US.

O'Grady, J., Lynch, P., \& Kearney, P. (1985). Voluntary feed intake by lactating sows. Livestock production science, 12(4), 355-365.

Palou, M., Picó, C., \& Palou, A. (2018). Leptin as a breast milk component for the prevention of obesity. Nutrition reviews, 76(12), 875-892.

Pinotti, L., \& Rosi, F. (2006). Leptin in bovine colostrum and milk. Hormone and metabolic research, 38(2), 89-93.

Quesnel, H., Mejia-Guadarrama, C. A., Pasquier, A., Dourmad, J.-Y., \& Prunier, A. (2005). Dietary protein restriction during lactation in primiparous sows with different live weights at farrowing: II. Consequences on reproductive performance and interactions with metabolic status. Reproduction nutrition development, 45(1), 57-68. 
Quesnel, H., Etienne, M., \& Père, M.-C. (2007). Influence of litter size on metabolic status and reproductive axis in primiparous sows. Journal of animal science, 85(1), 118-128.

Quiniou, N., \& Noblet, J. (1999). Influence of high ambient temperatures on performance of multiparous lactating sows. Journal of animal science 77(8), 2124-2134.

Sadr Dadres, G., Whitaker, K. M., Haapala, J. L., Foster, L., Smith, K. D., Teague, A. M., Jacobs Jr., D. R., Kharbanda, E. O., McGovern, P. M., \& Schoenfuss, T. C. (2019). Relationship of Maternal Weight Status Before, During, and After Pregnancy with Breast Milk Hormone Concentrations. Obesity, 27(4), 621-628.

Salcedo, J., Frese, S., Mills, D., \& Barile, D. (2016). Characterization of porcine milk oligosaccharides during early lactation and their relation to the fecal microbiome. Journal of dairy science, 99(10), 7733-7743.

Sánchez, J., Oliver, P., Miralles, O., Ceresi, E., Picó, C., \& Palou, A. (2005). Leptin orally supplied to neonate rats is directly uptaken by the immature stomach and may regulate short-term feeding. Endocrinology, 146(6), 2575-2582.

Savino, F., Sardo, A., Rossi, L., Benetti, S., Savino, A., \& Silvestro, L. (2016). Mother and infant body mass index, breast milk leptin and their serum leptin values. Nutrients, 8(6), 383.

Schenkel, A. C., Bernardi, M. L., Bortolozzo, F. P., \& Wentz, I. (2010). Body reserve mobilization during lactation in first parity sows and its effect on second litter size. Livestock science, 132(1-3), 165-172.

Van den Brand, H., Dieleman, S., Soede, N., \& Kemp, B. (2000). Energy balance of lactating primiparous sows as affected by feeding level and dietary energy source. American society of animal science, 78(6), 1520-1528

Wattanakul, W., Rooke, J., Stewart, A., English, P., \& Edwards, S. (2007). Effect of milk deprivation during the lactation period on performance and digestive enzyme activities of the piglets following weaning. Animal, 1(3), 381-387.

Wolter, B., \& Ellis, M. (2001). The effects of weaning weight and rate of growth immediately after weaning on subsequent pig growth performance and carcass characteristics. Canadian journal of animal science, 81(3), 363-369.

Yang, Y., Heo, S., Jin, Z., Yun, J., Choi, J., Yoon, S., Park, M. S., Yang, B. K., \& Chae, B. (2009). Effects of lysine intake during late gestation and lactation on blood metabolites, hormones, milk composition and reproductive performance in primiparous and multiparous sows. Animal reproduction science, 112(3), 199-214.

Zhong, H., Wang, P., Song, Y., Zhang, X., Che, L., Feng, B., Lin, Y., Xy, S., Li, J., \& Wu, D. (2018). Mammary cell proliferation and catabolism of adipose tissues in nutrition-restricted lactating sows were associated with extracellular high glutamate levels. Journal of animal science and biotechnology, 9(1), 78. 


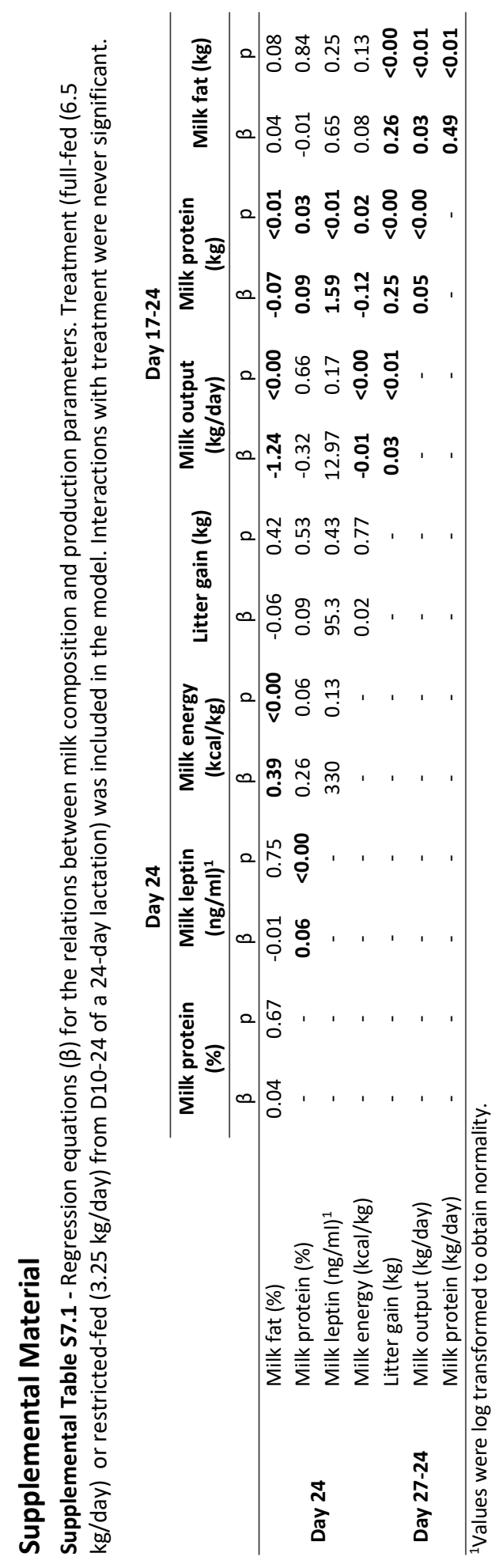




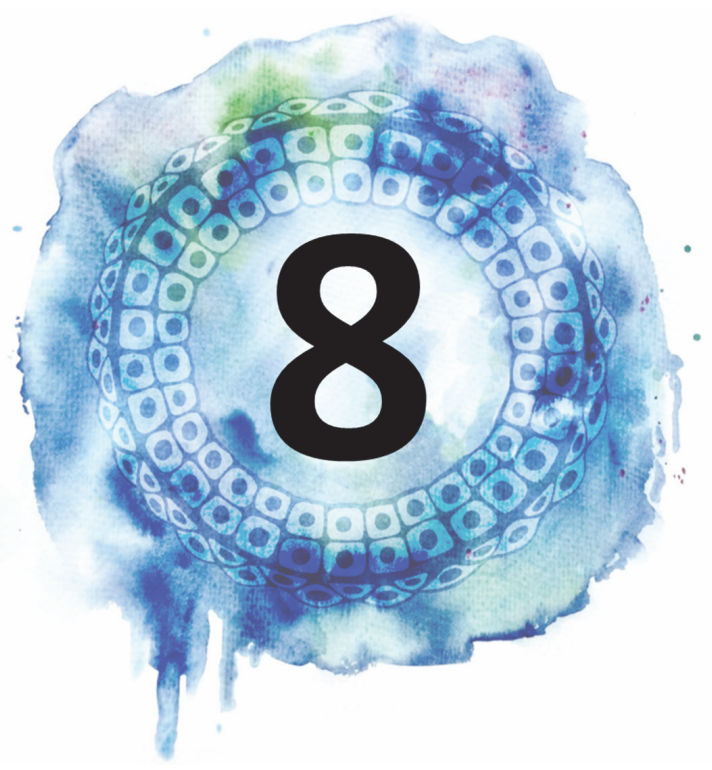

General Discussion 



\section{Introduction}

Modern sow lines have been selected for increased litter size, however, this increase in litter size is associated with an increase in piglet mortality (reviewed by Rutherford et al., 2013). Two factors which may explain this higher piglet mortality are lower piglet birth weights and higher within-litter variation in piglet birth weight (Quesnel et al., 2008; Quiniou et al, 2002; Wientjes et al., 2012a). Piglets with a lower birth weight have an increased risk of dying as they have less energy reserves and thermoregulatory capacity at birth and have a disadvantage in competing with larger littermates at the udder (Tuchscherer et al., 2000). The latter is especially true in litters with a higher within-litter variation in piglet birth weight. The main hypothesis of this thesis is that these factors, the lower piglet birth weights and the higher within-litter variation, are (at least partly) explained by suboptimal ovarian conditions in sows before conception. In addition, selection for litter size has increased the metabolic demands of sows during lactation due to the necessary higher milk production. During lactation antral follicles develop that contain the oocytes that will give rise to the next litter. Antral follicular development is highly influenced by the metabolic state of the sow (Quesnel et al., 1998; Zak et al., 1997), and the higher metabolic demands in modern sows may therefore negatively affect follicular developmental competence. Despite of this knowledge, it is at present not clear which metabolic processes play the most significant roles in influencing follicular developmental competence in modern sows.

Elucidating physiological characteristics which determine follicular developmental competence and understanding how follicular development is influenced by the metabolic state of the sow, could increase our understanding of the origin of reproductive problems in pigs and other mammals. Therefore, the aims of this thesis were:

1) To establish physiological and molecular characteristics of follicular developmental competence in sows by unravelling physiological processes that correlate with antral follicle size and oocyte health and 2) to better understand physiological relations between the metabolic state of sows and follicular developmental competence.

In this thesis, results from two pig studies are described. The first study describes follicular development and oocyte quality of multiparous sows at the onset of the follicular phase of the cycle (weaning), where antral follicles are stimulated to grow out to ovulatory sizes. The second study describes follicular development and oocyte quality in primiparous sows at the mid-follicular phase ( $48 \mathrm{~h}$ after weaning). The experimental design of these two experiments is summarized in Table 8.1. 
Table 8.1 - Experimental design of the two pig experiments that are described in this thesis.

\section{Experiment $1 \quad$ Experiment 2}

\begin{tabular}{lll}
\hline Parity & Multiparous (3-5) & Primiparous (1) \\
Lactation & 26 days & 24 days \\
Lactation feed allowance & Ad libitum (N=29) & Full-fed: $6.5 \mathrm{~kg} /$ day from D10-24 (N=18) \\
& & Restricted-fed: $3.25 \mathrm{~kg} /$ day from D10-24 (N=18) \\
Ovaries studied & Onset of follicular phase & Mid-follicular phase \\
\hline
\end{tabular}

Firstly, in order to establish physiological characteristics of follicular developmental competence in sows (Aim 1), granulosa cell gene and protein expression and follicular fluid composition was assessed in relation to two follicular competence markers, average follicle size and cumulus-oocyte complex (COC) morphology. Physiological characteristics of follicular competence that have been identified in this thesis (Chapter 3, 4 and 5) are summarized in Fig. 8.1 and will be further discussed in paragraph 8.1. In addition, potential implications of these findings will be discussed.

Secondly, this thesis describes the physiological relations between the metabolic state of the

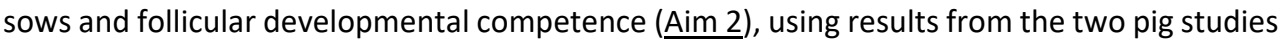
that were performed (Table 8.1). The first study investigated relations between metabolic parameters (e.g. body weight, backfat depth, plasma insulin-like growth factor 1 (IGF1) content) and follicular development in multiparous sows at the start of the follicular phase (weaning; Chapter 2) and the second study investigated effects of weight loss due to lactational feed restriction on metabolic parameters during lactation and follicular development in primiparous sows at the mid-follicular phase (48h after weaning) (Chapter 6 and 7). An overview of the physiological relations between the sows' metabolic state and follicular developmental competence is shown in Fig. 8.1. In paragraph 8.2, effects of weight loss during lactation on reproductive performance and follicular development in modern sows will be further explored and discussed by means of additional data analyses.

Thirdly, weight loss during lactation is due to energy mobilization from adipose tissue and skeletal muscle to sustain milk production. As both are simultaneously mobilized, it is difficult to establish which of the two is responsible for impairing follicular developmental competence. This is important, as modern sows have a high genetic merit for leanness, and body reserves in terms of fat and protein content have changed. These changes in body composition may have consequences for sow metabolism during lactation and consequently, reproductive performance. In this thesis, relations between backfat loss and muscle depth loss during lactation, their associated metabolic intermediates in the blood and follicular developmental competence were assessed and results are summarized in Fig. 8.1. In paragraph 8.3, differential effects of energy mobilization from various sources on follicular developmental competence will be further discussed. Potential implications of the findings of this thesis and recommendations for future research are discussed in paragraph 8.4. Finally, general conclusions are given in paragraph 8.5. 


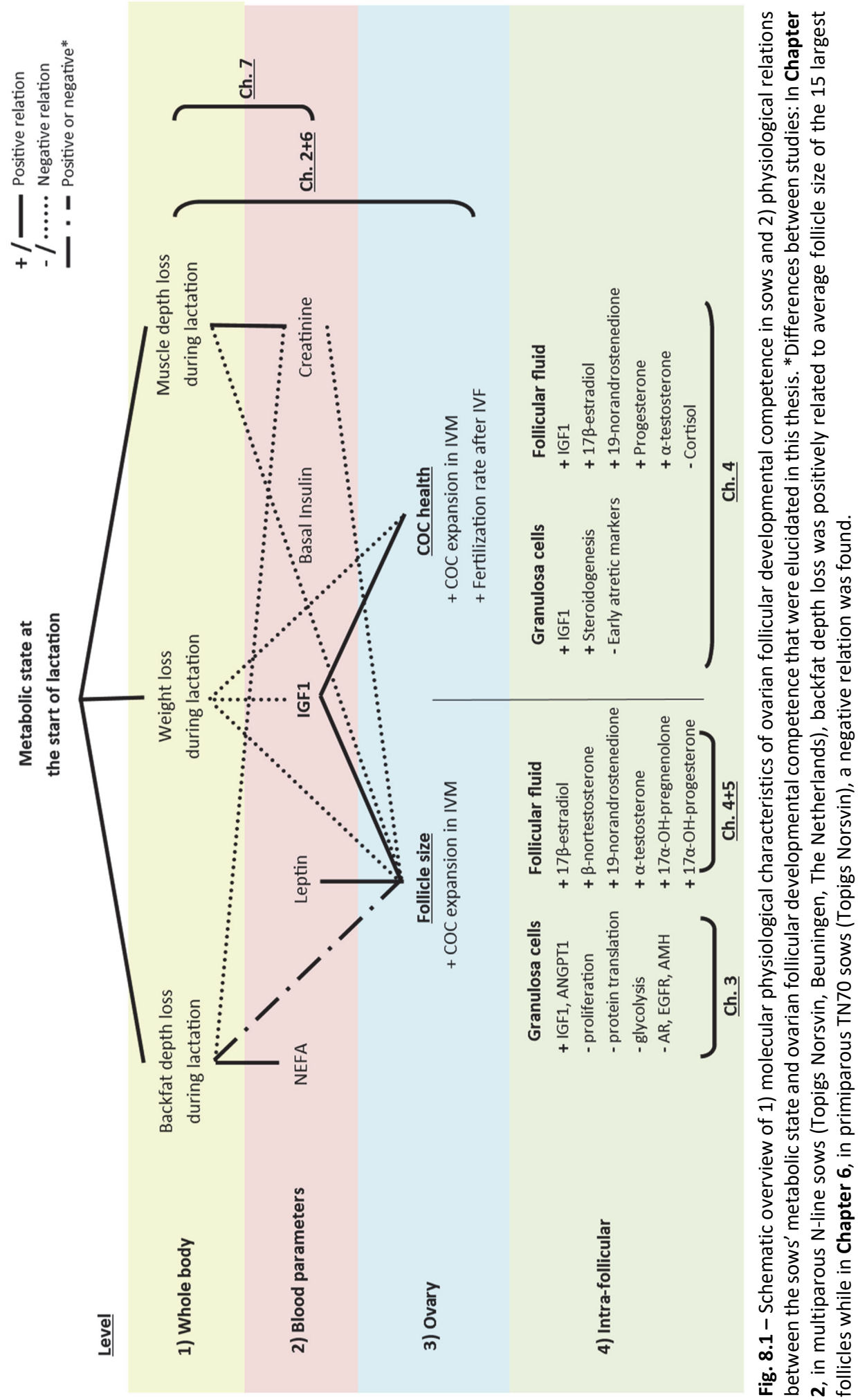




\subsection{Physiological characteristics of follicular developmental competence}

\subsubsection{Characteristics associated with follicle size}

Recruitment of follicles from the antral follicle pool at the onset of the follicular phase results in the growth and development of small antral follicles into pre-ovulatory follicles, which will finally ovulate (reviewed by Oktem and Urman, 2010). Average follicle size of a female at a specific stage of the follicular phase of the cycle, could therefore reflect the developmental stage of the follicle pool, which in turn, determines follicular developmental competence. In this thesis, follicular developmental competence is defined as the competence of a follicle to yield a mature oocyte which can successfully develop into healthy offspring after fertilization. In Chapter 2, 4, 5 and 6, follicular characteristics of sows with a different average follicle size are compared to identify characteristics of follicular developmental competence. For all of these analyses, only the largest follicles in the pool are assessed, as there are many antral follicles on an ovary, but not all of these antral follicles are expected to ovulate. Focusing on these largest follicles increases the chance that the subset of follicles studied indeed yield the oocytes that will ovulate, be fertilized and develop into offspring. Importantly, in this thesis, comparisons are always made between sows that were in the same stage of the estrous cycle.

A

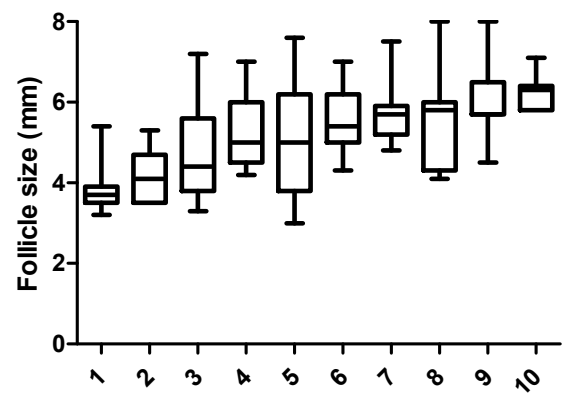

Multiparous sows at the onset of follicular phase
B

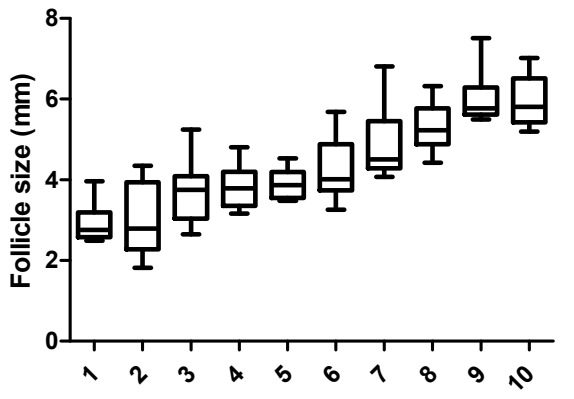

Primiparous sows at the mid-follicular phase

Figure 8.2 - Follicle size of the 15 largest follicles of the right ovary of a subset of $\mathbf{A} 10$ (1-10) multiparous sows at the onset of the follicular phase (first experiment) and B 10 full-fed primiparous sows at the mid-follicular phase (second experiment). Follicle size was measured using different methods in experiment 1 versus 2 (see Chapter $\mathbf{2}$ and $\mathbf{6}$ for details) and can therefore not be directly compared.

The average follicle size of the 15 largest follicles was $5.0 \pm 0.1$ (3.8-6.3) $\mathrm{mm}$ for the multiparous sows at the onset of the follicular phase (Chapter 2 ) while the average follicle size was $4.2 \pm 0.2$ (2.7-6.1) $\mathrm{mm}$ in the full-fed primiparous sows at the mid-follicular phase (Chapter 6). This shows that there is a high degree of variation in average follicle size between sows, which is already present from the onset of the follicular phase onwards. Next to differences between sows in average follicle size of the pool, there is also a considerable degree of heterogeneity in follicle size within the antral follicle pool of a sow. In this thesis, the range in follicle size of the 15 largest follicles was $3.3 \pm 0.2(1.3-5.4) \mathrm{mm}$ for the multiparous sows at the onset of the 
follicular phase (Chapter 2) and $2.0 \pm 0.2$ (1.1-2.6) $\mathrm{mm}$ in the full-fed primiparous sows at the mid-follicular phase (Chapter 6). Heterogeneity in follicle size has previously been identified in pre-ovulatory follicle pools of sows (Hunter and Wiesak, 1990). In this thesis it is shown that a high degree of heterogeneity in follicle size is already present from the onset of the follicular phase onwards. The average follicle size and the range in follicle size of the 15 largest follicles of a subset of sows $(\mathrm{N}=10)$ of both experiments are shown in Fig. 8.2.

In the multiparous sows at the mid-follicular phase, a larger average follicle size of the pool was not related to the percentage morphological healthy COCs, but was related to higher COC expansion rates after $22 \mathrm{~h}$ of in vitro maturation (IVM). This COC expansion is critical for maturation of the oocyte (reviewed by Russel and Robker, 2007). A larger average follicle size was also related to higher follicular fluid IGF1 levels (Chapter 6). In addition, a larger average follicle size was related to increased ovarian steroidogenesis. To be more specific, a higher average follicle size was related to higher follicular fluid $17 \beta$-estradiol levels in both primiparous sows at the mid-follicular phase as well as multiparous sows at the onset of the follicular phase (Chapter 4 and 5). At the mid-follicular phase, average follicle size was also related to higher $17 \alpha-\mathrm{OH}$-pregnenolone and $17 \alpha-\mathrm{OH}$-progesterone levels. All of these follicular characteristics that have been associated with average follicle size of the pool, are indicative of follicular maturation. This suggests that average follicle size determines the developmental stage of the follicle pool in sows which are at a specific stage of the estrous cycle.

Heterogeneity in follicle size within the follicle pool was further investigated by comparing gene and protein expression of smaller and larger follicles within the follicle pool of the 10 largest healthy follicles (Chapter 3). For the transcriptome analysis, follicles were classified as either healthy or unhealthy based on a cleaved-Caspase 3 staining (a marker for apoptotic cells). This allows for the study of healthy follicles only, which increases the predictive value for oocyte developmental competence and consequently, for reproductive outcome. Smaller follicles in the pool were more proliferative, possibly due to a higher expression of androgen receptor (AR) and epidermal growth factor receptor (EGFR). Expression of these receptors enables mitogenic actions of oocyte secreted factors and androgens produced by the theca cells to induce granulosa cell proliferation (Hickey et al., 2005; Sasseville et al., 2010). In contrast to smaller follicles, larger follicles were more differentiated as they showed a higher expression of IGF1 and angiopoetin 1 (ANGPT1). IGF1 is considered a follicular maturation marker (May and Schomberg, 1984) and ANGPT1 is an angiogenic factor that is necessary for blood vessel maturation and its expression increases in granulosa cells in late stages of follicular development in preparation of ovulation and luteinization (Robinson et al., 2009; Hayashi et al., 2003; Hazzard et al., 1999). In addition, higher follicular ANGPT1 and IGF1 expression have both been associated with improved human in vitro fertilization (IVF) outcomes (Faraj et al., 2017; Vural et al., 2016). Together this indicates that larger follicles in 
the pool are more mature and have a higher developmental competence as compared to the smaller follicles in the pool. Additional data analysis was performed for this discussion, to investigate relations between individual follicle size and granulosa cell gene expression of selected genes. Granulosa cell IGF1 expression was related to a larger follicle size, while ANGPT1 expression tended to be related to a larger follicle size. In addition, granulosa cell EGFR was related to a smaller follicle size, and AR and AMH expression tended to be related to a smaller follicle size (Fig. 8.3). The results of this analysis further highlight these factors as promising markers for follicular developmental competence, already at the onset of the follicular phase onwards.
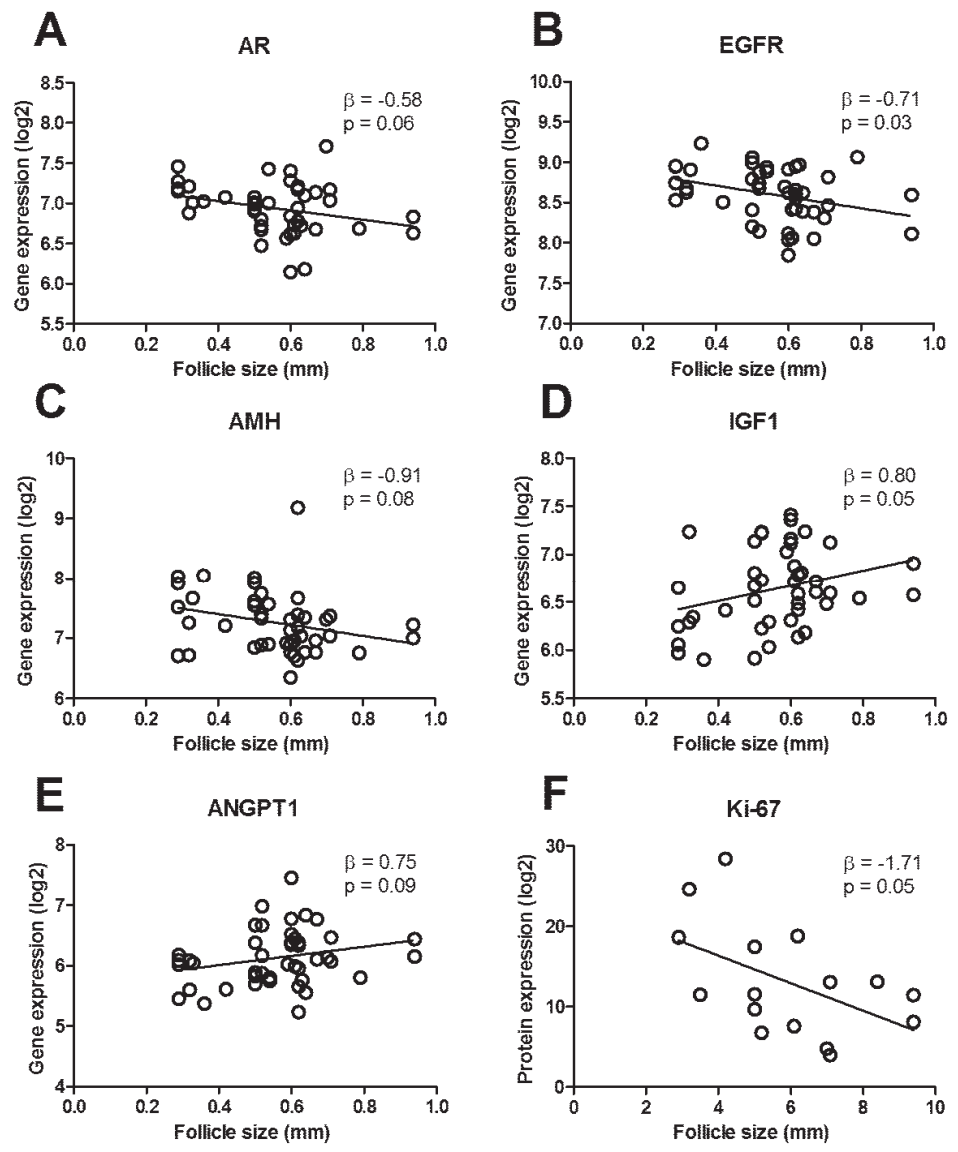

Figure 8.3 - Relations between follicle size $(\mathrm{mm}$ ) (of follicles of the 10 largest healthy follicles of each sow) and gene expression ( $\log 2)$ of androgen receptor (AR) (A), epidermal growth factor receptor (EGFR) (B), anti-Müllerian hormone (AMH) (C), insulin-like growth factor 1 (IGF1) (D), angiopoetin 1 (ANGPT1) (E), as determined via whole-genome microarray analysis, and protein expression of the proliferation marker Ki-67 (F), as determined by immunohistochemistry. See Chapter $\mathbf{3}$ for experimental details. Relations were estimated using proc mixed in SAS 9.4. 


\subsubsection{Characteristics associated with COC health}

Successful oocyte maturation, fertilization and development into an embryo, depends on the quality of the oocyte and its surrounding cumulus cells, the cumulus oocyte complex (COC). Morphological classifications of COCs that have been tested as predictors for oocyte developmental competence, include the number of cumulus cell layers (Yuan et al., 2005), the darkness of the ooplasm (Nagano et al., 2006) and the number of oocyte anomalies (Mikkelsen et al., 2001). These studies, however, were not always successful in predicting COC competence as shown by e.g. nuclear maturation of the oocytes after IVM or blastocyst formation rates after IVF, or pregnancy rates (reviewed in Wang and Sun, 2006). Alvarez et al. (2009) combined the abovementioned morphological parameters, as COCs with an intact cumulus layer and a normal shaped oocyte (healthy) or COCs with a degraded cumulus or degenerated oocyte (unhealthy). They found that these morphologically healthy COCs had the highest cytoplasmic and nuclear maturation rates after IVM and IVF. Therefore, in this thesis, follicular characteristics of sows with a high vs. low percentage of $\mathrm{COC}$ health were compared to identify characteristics for follicular developmental competence. A representative image of healthy and unhealthy COCs, according to the classification used in this thesis, is shown in Fig. 8.4A.

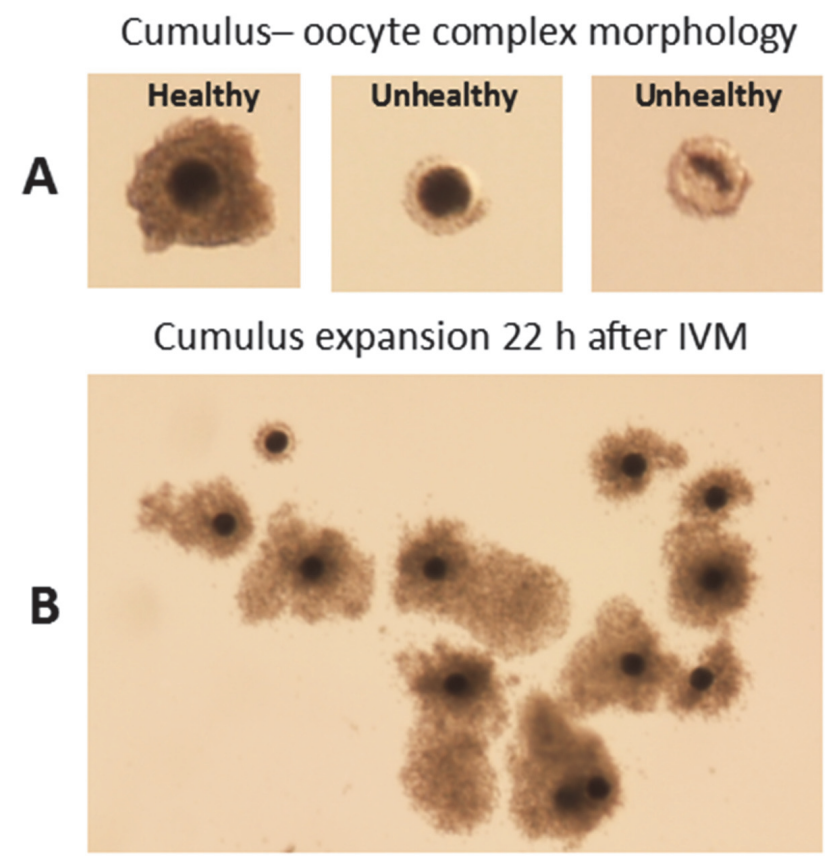

Figure 8.4 - Representative image of (A) morphology of cumulus-oocyte complexes (healthy and unhealthy) at $0 \mathrm{~h}$ and (B) cumulus expansion $22 \mathrm{~h}$ after in vitro maturation (as a measure of developmental competence of COCs) of one full-fed primiparous sow, of which the ovary was obtained 48 h after weaning (Chapter 6) 
In primiparous sows at the mid-follicular phase, a higher percentage of morphologically healthy COCs of the largest 15 follicles was related to more COC expansion $22 \mathrm{~h}$ after IVM (Fig. 8.4B) and to fertilization rate as measured 24h after IVF (Chapter 6). In addition, pooled follicular fluid of follicles with a lower percentage healthy COCs contained less $17 \beta$-estradiol and more cortisol (Chapter 4), a combination which is indicative of lower follicular competence (Grupen and Armstrong, 2010; Guthrie and Cooper, 1996; Westhof et al., 1991; Yuan et al., 2016). This supports the use of the current morphological classification into healthy or unhealthy COCs to predict follicular developmental competence of the pool.

To further establish physiological characteristics of follicular developmental competence in sows, granulosa cells of sows with a high vs. low percentage healthy COCs were isolated for transcriptome analysis (Chapter 4). For each of the analysed sows, these granulosa cells were isolated from the 3 largest and 3 smallest follicles of the pool of 10 largest healthy follicles. Several genes were identified which had a differential expression in sows with a high vs. low percentage healthy COCs. Sows with a high percentage healthy COCs showed a higher expression of genes involved in steroidogenesis, a lower expression of genes involved in early follicular atresia and a higher expression of IGF1 (Chapter 4). IGF1 could be important in regulating follicular competence, as IGF1 is widely recognized as an anti-apoptotic factor (Guthrie et al., 1998) which is needed to stimulate follicular growth and steroidogenesis (Xia et al., 1994). When granulosa cell transcriptomes of only the 3 largest follicles was compared for sows with a high vs. low percentage COCs, 106 unique genes were differentially expressed $(F D R \leq 0.2 ; F C \geq 1.2)$. For the 3 smallest follicles, a total of 61 unique genes was differentially expressed between sows with a high vs. low percentage healthy COCs, which all overlapped with the differentially expressed genes of the large follicles. This indicates that both the larger and smaller follicles in the pool contribute to differences in granulosa cell transcriptomes between sows with a high vs. low percentage healthy COCs.

In Chapter 4, we made comparisons between sows based on the percentage of healthy COCs of the 15 largest follicles. This only allows for comparisons of follicular characteristics as an average of the follicle pool. However, it would be interesting to study follicular steroid profiles in relation to follicle size, $\mathrm{COC}$ morphology and granulosa cell processes on an individual follicle level. In mono-ovulatory mammals (e.g. human, bovine) with a large dominant follicle, individual follicles can be assessed for e.g. follicular fluid steroid and protein content (Summers et al., 2014; Walters et al., 2019) which can subsequently be associated with IVF outcome (Walters et al., 2019). When future follicular fluid analysis techniques become even more sensitive, this individual follicle analysis may also be applied for poly-ovulatory animals such as sows. This will provide more information about variation in follicular steroid production and oocyte developmental competence within the follicle pool of a sow. 


\subsubsection{Implications}

Results of this thesis show that a high degree of heterogeneity in average follicular development and oocyte competence exists, already at the early and mid-follicular phase in follicles that are expected to become ovulatory follicles. This heterogeneity is present between sows which are in the same stage of the oestrus cycle but is also present within the follicle pool of a sow. This is evident from the large differences in both gene and protein expression of granulosa cells of different-sized follicles within the same follicle pool. This heterogeneity in follicular granulosa cells may have consequences for heterogeneity in oocyte quality, as granulosa cells provide the oocyte with the necessary growth factors, nutrients and metabolic intermediates allowing the oocytes to obtain their full developmental competence (Jiang et al., 2010; Sugiara et al., 2005). Furthermore, heterogeneity in follicular development may influence the size and quality of the corpora lutea which develop from the follicles after ovulation and are essential to sustain early pregnancy. As fertilization rates in sows are close to $100 \%$ (Spötter and Distl, 2006), almost all oocytes of the ovulatory follicles will be fertilized and develop into an embryo. Therefore, it seems reasonable to assume that heterogeneity in follicular development of the follicles that ovulate will influence (variation in) subsequent early embryonic development. Early embryonic development is crucial as it determines early survival but also placenta size and thereby the size of the implantation site in the uterus and nutrient supply to the embryo in later stages of pregnancy (Chen and Dziuk, 1993; Ford et al., 2002; Geisert et al., 1982). A high number of embryos of variable quality, together with the limited uterine capacity, may result in high pre-natal mortality which may negatively influence piglet birth weights, which elevates piglet mortality. Of course, embryonic development in sows is also influenced by other factors than oocyte quality, such as the quality of the sperm, the condition of the fallopian tube and their interaction (reviewed by Brüssow et al., 2008; Simon et al., 2014; de Vos et al., 2003) next to the already mentioned sow uterine capacity (reviewed by Fowden et al., 2009). These factors are not investigated in this thesis and are therefore not further discussed. However, it may be expected that events that take place before the pre-implantation period play a very important role in determining (variation) in embryo quality and piglet birth weight. This is exemplified by the high degree of variation in embryo development that already exists in the pre-implantation period (Pope and First, 1985), even as early as the 1- to 4- cell stage (Xie et al., 1990).

Increased knowledge of factors that differ between smaller and larger follicles in the presumptive ovulatory follicle pool also has potential implications for human IVF protocols. Human IVF treatment includes administration of supra-physiological doses of folliclestimulating hormone (FSH) to induce growth of multiple ovarian follicles, after which a luteinizing hormone (LH) agonist, human chorionic gonadotropin (hCG), is administered to induce oocyte maturation. Subsequently, the mature follicles are retrieved by follicle aspiration which may be followed by IVF or intracytplasmic sperm injection (ICSI). By applying this type of controlled ovarian hyper-stimulation, not only the largest, but also the smaller, 
less mature follicles in the antral follicle pool are recruited and selected to develop into preovulatory follicles, after which these follicles are aspirated for oocyte retrieval. Including only the largest, most mature, follicles for IVF, may increase pregnancy rates. Perhaps, similar mechanisms also play a role in sows, which are selected for a large litter size. As a correlated response to applied selection strategies to increase litter size, ovulation rate has considerably increased (Da Silva et al., 2017). These increased ovulation rates are probably the result of increased follicular recruitment and selection. Indeed, sows with a high ovulation rate have higher FSH levels during mid and late luteal and peri-ovulatory stages (Knox et al., 2003) and a higher pre-ovulatory FSH surge and post-ovulatory FSH rise (42 hours after the primary FSH surge; Kelly et al., 1988). In addition, a positive correlation was found between ovulation rate and mean and peak FSH levels in the period from weaning to oestrus in first parity sows (Shaw and Foxcroft, 1985). FSH is essential for follicular recruitment and can prevent follicular atresia (Hirshfield and Midgley, 1978; Chun et al., 1994), and may therefore rescue follicles that normally would have degenerated. Therefore, increased follicular recruitment in sows with a high ovulation rate could lead to additional selection of smaller, less mature follicles into the pre-ovulatory follicle pool, which may have negative consequences for reproductive outcome and may partially explain current reproductive problems associated with high prolificacy. To fully understand the underlying mechanism of these described reproductive problems in modern sows, further investigation of relations between follicular characteristics and subsequent reproductive outcome is needed.

\subsection{Summarizing physiological characteristics of follicular developmental competence}

The results of our studies show a high degree of variation in follicle size and COC health between sows in the same stage of the oestrus cycle, but also high variability within the follicle pool of follicles which are expected to ovulate. This variation may result in variable oocyte quality and early embryonic development. Furthermore, in this thesis it was confirmed that both average follicle size of sows in the same stage of the oestrus cycle as well as percentage healthy COCs of the 15 largest follicles were positively related to follicular developmental competence, as was shown by increased COC expansion after 22h of IVM. In addition, the percentage healthy COCs was also related to higher fertilization rates after IVF. Subsequently, several follicular characteristics could be identified which are related to average follicle size and COC health (Aim 1 of this thesis). Of special interest is IGF1, as IGF1 gene expression in granulosa cells was higher in larger follicles and was higher in sows with a higher percentage of healthy COCs. My research thus showed the essential role of granulosa cell IGF production in follicle size and developmental competence. 


\subsection{Influence of weight loss during lactation on reproductive performance, litter characteristics and follicular developmental competence in modern sows}

8.2.1 Influence of weight loss on reproductive performance and litter characteristics Pig production profitability highly depends on the number of piglets produced per sow per year. To increase the number of produced piglets, modern sow lines have been genetically selected to increase sow litter size. As mentioned previously, this results in high metabolic demands of sows during lactation, as sows need to produce a large amount of milk to feed the large number of piglets. These high metabolic demands during lactation influence reproductive performance in the next litter. Previous studies found that weight loss during lactation can negatively affect reproductive outcome and next litter characteristics in sows when it exceeds 10-12\% of initial body weight at farrowing (Schenkel et al., 2010; Thaker and Bilkei, 2005). High weight loss is associated with longer weaning-to-oestrus intervals (WOI), reduced successive farrowing rates, lower embryo weights and survival at various stages of pregnancy resulting in smaller litter sizes and increased within-litter variation in piglet birth weight (Hoving et al., 2012; Patterson et al., 2011; Schenkel et al., 2010; Thaker and Bilkei, 2005; Vinsky et al., 2006; Wientjes et al., 2013).

Next to selection for increased litter size, sows have also been selected for a shorter weaningto-oestrus interval (WOI), which can further increase the number of piglets produced per year. This shortening of the WOI over the last few decades can be seen in Table 8.5, which describes several studies performed over the last decades, which applied feed restriction during lactation and studied its effects on WOI. Average WOI is longer in older studies as compared to more recent studies (Table 8.5). Follicular development and therefore the duration of WOI is controlled by the LH release pattern before and after weaning (Shaw and Foxcroft, 1985). Lactational weight loss probably influences this LH pulsatility, as feed restriction during lactation reduces the frequency of LH pulsatility (Quesnel et al., 1998) and reduces mean and basal LH levels around weaning (Kauffold et al., 2008), subsequently prolonging WOI. Early studies show that feed restriction and associated lactational weight loss can prolong WOI, however, this effect is much less pronounced or absent in later studies suggesting that weight loss may not prolong WOI in modern sows (see Table 8.5 for an overview). This indicates that the follicle pools of these modern sows have less time to recover from negative influences of weight loss during lactation. In turn, this may negatively affect developmental competence of the follicle pool that will finally ovulate and give rise to the next litter of piglets. This hypothesis is supported by Kemp et al. (2018), who describes that feed restriction in modern sows does not prolong WOI anymore, while it has a larger negative effect on embryo survival at day $28 / 35$ of pregnancy. 
Table 8.5 - Effect of feed intake level (high or low) on relative weight loss (\%) and weaning-to-oestrus interval (WOI). Adapted from Kemp et al., (2018).

\begin{tabular}{cccccclll}
\multicolumn{2}{l}{ Weight loss (\%) } & \multicolumn{2}{c}{ WOI } & \multicolumn{2}{c}{ Feed restriction } & Lactation & Parity & Reference \\
\hline High & Low & High & Low & Level & Duration & & & \\
\hline 5.3 & 22.0 & $6.6^{\text {a }}$ & $19.9^{\mathrm{b}}$ & $50 \%$ & Day 1-32 & 32 days & 1 & King and Williams, 1984 \\
7.8 & 19.1 & $4.3^{\text {a }}$ & $5.8^{\text {b }}$ & $50 \%$ & Day 1-35 & 35 days & 2 & Kirkwood et al., 1987 \\
7.2 & 13.9 & $6.9^{\text {a }}$ & $8.9^{\text {b }}$ & $50 \%$ & Day 1-28 & 28 days & 2 & Kirkwood et al., 1990 \\
7.4 & 18.3 & $5.9^{\text {a }}$ & $7.3^{\text {b }}$ & $50 \%$ & Day 1-28 & 28 days & 2 & Baidoo et al., 1992 \\
5.8 & 13.4 & $3.7^{\text {a }}$ & $5.6^{\text {b }}$ & $50 \%$ & Day 1-21 & 28 days & 1 & Zak et al., 1997 \\
5.8 & 10.9 & $3.7^{\text {a }}$ & $5.1^{\text {b }}$ & $50 \%$ & Day 22-28 & & & \\
9.1 & 22.1 & $4.2^{\text {a }}$ & $6.3^{\text {b }}$ & $50 \%$ & Day 1-28 & 28 days & 1 & Zak et al., 1998 \\
$8.7^{2}$ & $13.9^{2}$ & 5.1 & 5.7 & $75 \%$ & Day 1-21 & 21 days & 1 & van den Brand et al., 2000 \\
2.7 & 3.1 & 5.3 & 5.4 & $50 \%$ & Day 14-21 & 21 days & 1 & Vinsky et al., 2006 \\
3.8 & 10.5 & 5.0 & 5.3 & $67 \%$ & Day 14-21 & 21 days & 1 & Patterson et al., 2011 \\
\hline
\end{tabular}

${ }^{1}$ Relative weight loss expressed as a percentage of initial body weight at farrowing. ${ }^{2}$ Estimated using initial body weight at day 3 after farrowing.

To further investigate effects of lactational weight loss on reproductive outcome and litter characteristics in modern sows, a data set was obtained which included data on 431 TN70 sows (Topigs Norsvin, Beuningen, The Netherlands). The sows of this dataset were housed on two different farms in the Netherlands and were fed a gradually increasing amount of a lactation diet (9.75 MJ NE/kg, $8.4 \mathrm{SID}$ lysine) starting at $2 \mathrm{~kg} /$ day around farrowing and increasing to $6.66 \mathrm{~kg} /$ day from day 15 of lactation onwards (personal communication, Chris Opschoor, Topigs Norsvin). This dataset includes data on the body weight and backfat depth of sows during their first lactation and subsequent reproductive performance and litter characteristics of the second parity. Analysis of this data set show that around $30 \%$ of the sows on these farms had a relative lactational weight loss of more than $12 \%$ of their initial body weight at farrowing. These sows which lost more than $12 \%$ relative weight loss, had a slightly longer WOI (+0.2 days), a smaller litter size (-2.8 piglet) and reduced farrowing rate $(-8 \%, \mathrm{P}=$ 0.11 ) as compared to sows which lost less than $12 \%$ body weight (Table 8.6$)$. This corroborates earlier findings that lactational weight loss which exceeds $10-12 \%$ of initial body weight has negative consequences for reproductive performance in the next cycle (Hoving et al., 2010 and 2012; Schenkel et al., 2010; Thaker and Bilkei, 2005). The WOI was only increased from 4.5 to 4.7 days in this data set, which is similar to the small effect found in recent studies as shown in Table 8.5. It should be noted that different from the studies described in Table 8.5, these sows were not subjected to feed restriction. Therefore, differences in weight loss are most likely due to other causes than an involuntary lower feed intake such as the longer lactation period and higher number of piglets weaned (Table 8.6).

Next to effects of lactational weight loss on WOI and litter size, a higher weight loss due to a lower feed intake also affects litter weight gain during lactation (De Bettio et al., 2016; Zhong et al., 2018). In Chapter 7, it was shown that feed restriction negatively affects litter weight 
gain, presumably due to the fact that milk fat and milk protein production were reduced in the restricted-fed sows. Litter weight gain and subsequent weaning weight of the piglets highly influences post-weaning piglet growth (Wolter and Ellis, 2001). The influence of weight loss during lactation on milk production is therefore another factor that should be considered in regard to pig production profitability.

Together, results show that also in modern primiparous sows lactational weight loss negatively influences litter characteristics of the next litter also in modern primiparous sows. These negative effects of lactational weight loss on reproductive outcome may at least be partially explained by the negative influence of lactational weight loss on the development of the follicle pool and oocytes which will give rise to the next litter. The influence of lactational weight loss on follicular developmental competence in sows will be discussed in the following section 8.2.2. 


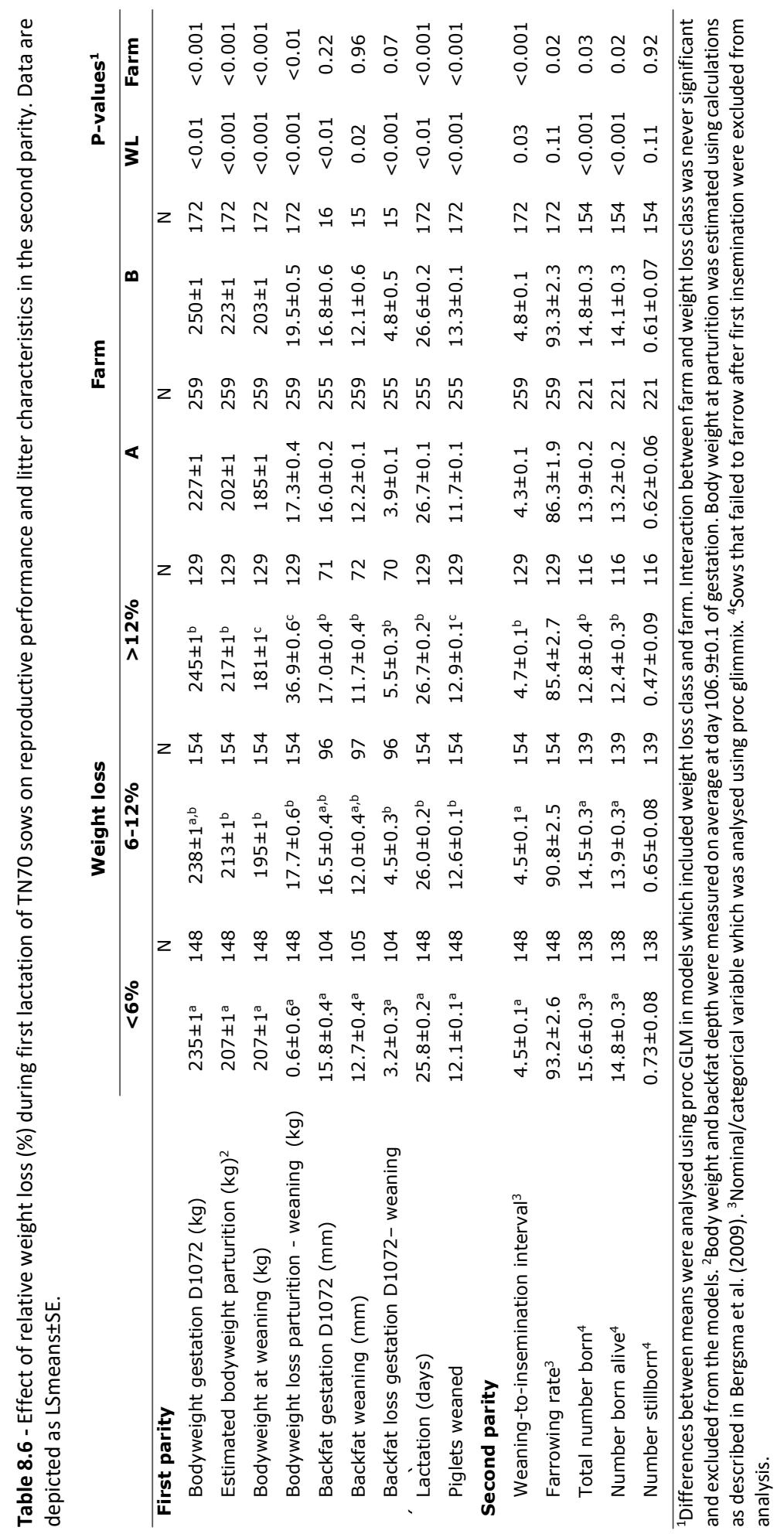




\subsubsection{Influence of weight loss on follicular developmental competence}

In Chapter 6, effects of lactational weight loss on post-weaning follicular and oocyte developmental competence were investigated in modern hybrid sows. Feed restriction was applied during the last two weeks of a 24-day lactation. This resulted in a relative weight loss of $19.6 \%$ for restricted-fed vs. $11.5 \%$ for full-fed sows during lactation. When assessing the pool of 15 largest antral follicles $48 \mathrm{~h}$ after weaning, feed restriction reduced average follicle size by $26 \%$ ( 3.1 vs. $4.2 \mathrm{~mm}$ ). Although feed restriction did not affect COC morphology, it did lower cumulus expansion rates after $22 \mathrm{~h}$ of IVM by $26 \%$ (175 vs. $237 \%$ ) and increase polyspermy by $89 \%$ ( 44 vs. $23 \%$ of the fertilized oocytes), the latter being a marker for immature oocytes (Van der Ven et al., 1985). In addition, follicular fluid of restricted sows had lower IGF1 (-56\%) and steroid levels (e.g. $\beta$-estradiol, progestins and androgens), which indicates that follicles of restricted sows are less competent to produce steroids and growth factors needed for oocytes to obtain full developmental competence.

There are only two other studies that address effects of weight loss due to feed restriction during lactation on follicular developmental competence in sows. These studies were performed over 20 years ago (Quesnel et al., 1998; Zak et al., 1997). In the study of Quesnel et al. (1998), primiparous sows received $50 \%$ feed restriction during a 28 -day lactation, which resulted in a weight loss of $21.2 \%$ of initial body weight at parturition for restricted-fed as compared to $8.2 \%$ for full-fed sows. Feed restriction resulted in a $15 \%$ reduction in average follicle size of the 10 largest follicles at weaning ( 2.8 vs. $3.3 \mathrm{~mm}$ for restricted-fed vs. full-fed) and a $23 \%$ reduction ( 3.7 vs. $4.8 \mathrm{~mm}$ in restricted-fed vs. full-fed) $48 \mathrm{~h}$ after weaning. It seems that especially feed restriction in the last week of lactation affected follicular developmental competence, as shown by the study of Zak et al. (1997), where primiparous sows either received feed restriction from day 1-21 or day 22-28 of a 28-day lactation period. Feed restriction in the last week of lactation resulted in more small follicles and relatively fewer large follicles $(-25 \%)$, a lower percentage of oocytes that reached metaphase II after $46 \mathrm{~h}$ of IVM (-69\%) and a lower capacity of the follicular fluid to mature slaughterhouse oocytes to metaphase II in vitro (-19\%) as compared to feed restriction from day 1-21. These above studies show that relative weight loss due to feed restriction is similar in older and modern sows. In addition, weight loss during lactation still highly influences follicular development and oocyte quality in modern sows.

However, reducing feed allowance to induce weight loss as done in these studies, not only reduces available energy, but also available (essential) amino acids, minerals and vitamins. For the restricted-fed primiparous sows in Chapter 6, it was calculated that based on their feed intake, these sows did not meet essential amino acid requirements of lysine, threonine and methionine (according to National Research Council, 2012). In addition, daily requirements of vitamin D3 and levels of several minerals (selenium, zinc, iron) were not met (data not shown). Inadequate levels of some of these diet components have been shown to negatively influence 
reproductive performance (e.g. reviewed by Smith and Akinbamijo, 2000; Xue et al., 2012). Next to this, feed restriction may cause increased stress levels (Amdi et al., 2013), negatively affecting reproductive performance parameters (reviewed by Einarsson et al., 2008). It is therefore not completely clear which of these factors, next to reduced feed energy, may have contributed to the negative effects of weight loss on follicular developmental competence. The influence of energy mobilization of different substrates during lactation (e.g. protein or fat) on follicular developmental competence will be described in paragraph 8.3.

\subsubsection{Influence of weight loss on transcriptome of granulosa cells of healthy follicles} In Chapter 3, granulosa cell transcriptome analysis was performed to assess differences in large and small follicles within the follicle pool of 10 largest healthy follicles at the onset of the follicular phase. Granulosa cells were investigated, as these are responsible for the production of growth factors and steroids needed for follicular growth and survival (reviewed by Matsuda et al., 2012) and therefore play an essential role in follicular development. The dataset obtained in Chapter $\mathbf{3}$ was used to perform additional analysis for this discussion, to further investigate effects of lactational weight loss on follicular developmental competence. The dataset included 8 sows from experiment 1, which were split in two groups: 4 multiparous sows with a high lactational weight loss $(12.0 \pm 2.2 \%)$ during a 26 -day lactation, and 4 sows with a low lactational weight loss $(4.7 \pm 1.5 \%)$, see Table 8.7 . Ovaries were collected within $2 \mathrm{~h}$ after weaning, and granulosa cells of the 3 largest and 3 smallest follicles of the pool of 10 largest healthy follicles of the right ovary were isolated using laser-capture microdissection (see Chapter 3 for experimental details). Body weight, follicle size and $\mathrm{COC}$ health of these two groups of sows is described in Table 8.7. Percentage healthy COCs of the 15 largest follicles of the left ovary and average follicle size of the 3 smallest and 3 largest selected healthy follicles of the right ovary was similar for both groups (Table 8.7). Granulosa cell transcriptomes of the sows with a high vs. low weight loss during lactation were compared.

Table 8.7 - Effect of relative lactational weight loss (low $=4.7 \pm 1.5 \%$ and high $=12.0 \pm 2.2 \%$ ) during a 26 day lactation. Data are presented as average (range).

\begin{tabular}{lcc} 
& Low weight loss $(\mathbf{N}=\mathbf{4})$ & High weight loss (N=4) \\
\hline Body weight gestation D108 & $263(233-284)$ & $291(266-305)$ \\
Body weight parturition (kg) & $232(204-261)$ & $257(234-279)$ \\
Body weight weaning (kg) & $221(190-250)$ & $226(211-245)$ \\
Weight loss (kg) & $11(8-14)$ & $32(19-62)$ \\
Weight loss (\%) & $4.7(3.1-7.0)$ & $12.0(7.2-22.1)$ \\
Average follicle size (mm) & $5.4(4.3-6.3)$ & $5.2(4.2-5.7)$ \\
Selected 3 largest follicles (mm) & $6.6(6.0-7.4)$ & $6.6(5.0-7.9)$ \\
Selected 3 smallest follicles (mm) & $4.8(3.0-6.2)$ & $4.2(3.4-5.1)$ \\
Healthy COCs (\%) & $77(40-100)$ & $69(50-100)$ \\
\hline
\end{tabular}

${ }^{1}$ Body weight was measured on average at day 108.3 \pm 0.3 . ${ }^{2}$ Start weight and end weight were estimated based on body weight 1 week before parturition and body weight at weaning according to Bergsma et al. (2009)

(Chapter 2). 
A total of 47 unique genes were differentially expressed (FDR $<0.1$ and FC $>1.2$ ) in granulosa cells of sows with high vs. low relative lactational weight loss. Pathway analysis of FDR $<0.2$ and $\mathrm{FC}<1.2$ genes revealed that 9 out of the 10 most significant pathways are involved in protein translation and the regulation of translation, e.g. ribosome biogenesis and mRNA degradation (Table 8.8).

Table 8.8 - Top 10 pathways of FDR $\leq 0.20$ genes in granulosa cells of multiparous sows (parity $3-5$ ) with a high weight loss $(12.0 \pm 2.2 \%, \mathrm{~N}=4)$ vs. low weight loss $(4.7 \pm 1.5 \%, \mathrm{~N}=4)$ during a 26 -day lactation.

\begin{tabular}{|c|c|c|c|c|}
\hline & Pathway ${ }^{1}$ & Number ${ }^{2}$ & Total $^{3}$ & FDR \\
\hline 1 & $\begin{array}{l}\text { Nonsense Mediated Decay (NMD) independent of the Exon } \\
\text { Junction Complex (EJC) }\end{array}$ & 20 & 101 & $6.8 \mathrm{E}-15$ \\
\hline 2 & Formation of a pool of free $40 \mathrm{~S}$ subunits & 19 & 106 & $6.8 \mathrm{E}-15$ \\
\hline 3 & Nonsense-mediated decay (NMD) & 21 & 124 & $6.8 \mathrm{E}-15$ \\
\hline 4 & $\begin{array}{l}\text { Nonsense mediated decay enhanced by the exon-junction } \\
\text { complex }\end{array}$ & 21 & 124 & $6.8 \mathrm{E}-15$ \\
\hline 5 & Viral mRNA translation & 19 & 114 & $6.8 \mathrm{E}-15$ \\
\hline 6 & SRP-dependent cotranslation protein targeting to membrane & 19 & 119 & $6.8 \mathrm{E}-15$ \\
\hline 7 & GTP hydrolysis and joining of the 605 ribosomal subunit & 19 & 120 & $6.8 \mathrm{E}-15$ \\
\hline 8 & Peptide chain elongation & 19 & 97 & $6.8 \mathrm{E}-15$ \\
\hline 9 & $\begin{array}{l}\text { L13a-mediated translational silencing of Ceruloplasmin } \\
\text { expression }\end{array}$ & 20 & 120 & $6.8 \mathrm{E}-15$ \\
\hline 10 & Eukaryotic translation termination & 19 & 106 & $6.8 \mathrm{E}-15$ \\
\hline
\end{tabular}

${ }^{1}$ Pathway analysis was performed using Reactome, ${ }^{2}$ Number of significantly different genes in the pathway, ${ }^{3}$ Total number of genes in the pathway.

Individual transcript analysis of the genes in these pathways revealed that granulosa cells of sows with high relative lactational weight loss have a higher expression of genes involved in protein translation (top 10 transcripts with a higher and lower expression in sows with a high weight loss during lactation are shown in Table 8.9 and Table 8.10, respectively). 


\section{General Discussion}

Table 8.9 - Top 10 FDR $\leq 0.1$ genes in granulosa cells with a higher expression in multiparous sows (parity $3-5)$ with high weight loss $(W L)(12.0 \pm 2.2 \%, N=4)$ vs. low weight loss $(4.7 \pm 1.5 \%, N=4)$ during a 26 day lactation. Top genes were selected based on highest fold-change (FC).

\begin{tabular}{|c|c|c|c|c|c|c|}
\hline \multirow[b]{2}{*}{ Gene symbol } & \multirow[b]{2}{*}{ Gene description } & \multirow[b]{2}{*}{ Accession $\mathrm{nr}$} & \multicolumn{2}{|c|}{ Log2 expression } & \multirow[b]{2}{*}{ FC } & \multirow[b]{2}{*}{ FDR } \\
\hline & & & $\begin{array}{l}\text { High- } \\
\text { WL }\end{array}$ & $\begin{array}{l}\text { Low- } \\
\text { WL }\end{array}$ & & \\
\hline BTBD9 & BTB domain containing 9 & XM_021098099 & 7.44 & 5.90 & 2.91 & $<0.001$ \\
\hline JARID2 & $\begin{array}{l}\text { Jumonji and AT-rich interaction domain } \\
\text { containing } 2\end{array}$ & XM_021100205 & 6.32 & 5.64 & 1.60 & $<0.001$ \\
\hline RPS23 & Ribosomal protein $\mathrm{S} 23$ & NM_213764 & 7.16 & 6.57 & 1.51 & 0.10 \\
\hline RPL34 & Ribosomal protein L34 & XM_005666943 & 7.50 & 6.91 & 1.51 & 0.03 \\
\hline RREB1 & Ras responsive element binding protein & XM_005665552 & 7.41 & 6.83 & 1.49 & $<0.001$ \\
\hline RAB11FIP2 & RAB11 family interacting protein 2 & XM_001927901 & 6.55 & 6.62 & 1.39 & 0.10 \\
\hline RPL5 & Ribosomal protein L5 & NM_001114284 & 7.60 & 7.14 & 1.38 & 0.02 \\
\hline ZNF217 & Zinc finger protein 217 & XM_005673023 & 6.70 & 6.31 & 1.31 & 0.05 \\
\hline RPSA & Ribosomal protein SA & NM_001037146 & 6.05 & 5.66 & 1.30 & 0.08 \\
\hline AKAP9 & A-kinase anchoring protein 9 & NM_001253824 & 7.06 & 6.67 & 1.30 & 0.02 \\
\hline
\end{tabular}

Table 8.10 - Top 10 FDR $\leq 0.1$ genes in granulosa cells with a lower expression in multiparous sows (parity $3-5)$ with high weight loss $(W L)(12.0 \pm 2.2 \%, N=4)$ vs. low weight loss $(4.7 \pm 1.5 \%, N=4)$ during a 26-day lactation. Top genes were selected based on highest fold-change (FC).

\begin{tabular}{|c|c|c|c|c|c|c|}
\hline \multirow[b]{2}{*}{ Gene symbol } & \multirow[b]{2}{*}{ Gene description } & \multirow[b]{2}{*}{ Accession $\mathrm{nr}$} & \multicolumn{2}{|c|}{ Log2 expression } & \multirow[b]{2}{*}{ FC } & \multirow[b]{2}{*}{ FDR } \\
\hline & & & $\begin{array}{l}\text { High- } \\
\text { WL }\end{array}$ & $\begin{array}{l}\text { Low- } \\
\text { WL }\end{array}$ & & \\
\hline SLA-3 & MHC class I antigen 3 & NM_001097427 & 5.54 & 6.68 & -2.20 & 0.02 \\
\hline LSG1 & $\begin{array}{l}\text { Large } 60 \text { s subunit nuclear export } \\
\text { GTPase } 1\end{array}$ & XM_021070074 & 6.90 & 8.00 & -2.14 & $<0.01$ \\
\hline ENSSSCT00000005783 & Unknown & - & 6.64 & 7.47 & -1.78 & 0.07 \\
\hline ACAD8 & $\begin{array}{l}\text { Acyl-CoA dehydrogenase family } \\
\text { member } 8\end{array}$ & NM_001243574 & 9.30 & 10.09 & -1.72 & 0.04 \\
\hline CCDC17 & Coiled-coil domain containing 17 & XM_021096729 & 5.94 & 6.66 & -1.66 & $<0.01$ \\
\hline ENSSSCT00000035758 & Unknown & - & 8.20 & 8.90 & -1.63 & 0.02 \\
\hline GLB1 & Galactosidase beta 1 & XM_021071553 & 8.38 & 9.06 & -1.60 & 0.05 \\
\hline KDM1A & Lysine demethylase $1 \mathrm{~A}$ & NM_001112687 & 5.78 & 6.46 & -1.60 & $<0.01$ \\
\hline ZFP36L1 & ZFP36 ring finger protein like 1 & XM_013989124 & 8.47 & 9.13 & -1.58 & 0.10 \\
\hline DECR1 & 2,4-dienoyl-CoA reductase 1 & NM_001190232 & 7.40 & 8.05 & -1.58 & 0.08 \\
\hline
\end{tabular}


For most of these genes, their function in female reproduction is not well investigated. Some of the most interesting genes will be highlighted. Granulosa cells of sows with high weight loss have a higher expression of A-Kinase Anchoring Protein (AKAP9), Zinc finger protein 217 (ZNF217) (see Table 8.3 for both) and Regulator Of Cohesion Maintenance, Homolog A (PDS5A, FDR $=0.03$ and $F C=1.29$ ). AKAP9, a A-kinase anchor protein that binds to the regulatory subunit of protein kinase $A$ to localize it to specific cellular compartments, is involved in centrosome function and microtubule organization (reviewed by College and Scott, 1999) and is essential for cell cycle progression (Keryer et al., 2003; Hu et al., 2016). In addition, AKAP9 plays an essential role in spermatogenesis and somatic cell-germ cell organization (Schimenti et al., 2013). PDS5A is essential for chromosome segregation during mitosis (Losada et al., 2005; reviewed by Peters, 2012; Mannini et al., 2010), while ZNF217 is a transcriptional regulator (reviewed by Cohen et al., 2015), and may be involved in the repression of cell differentiation (Krig et al., 2007). Finally, a lower expression of lysine demethylase $1 \mathrm{~A}$ (KDM1A) was found in granulosa cells of sows with a high versus low relative weight loss during lactation. KDM1A can demethylate histone $\mathrm{H} 3$ at two different sites and regulates gene transcription, and acts as a coactivator of androgen receptor dependent transcription (Metzger et al., 2016). Most of these described genes (e.g. PDS5A, AKAPO9 and ZNF217) are involved in regulating cell proliferation. Together, this may suggest that granulosa cells of sows with a high vs. low weight loss during lactation are more proliferative. To assess this, protein expression of Ki-67, a proliferation marker (Gerdes et al., 1984), was compared for granulosa cells of sows with high vs. low weight loss during lactation (see Chapter $\mathbf{3}$ for experimental details). Ki-67 protein expression tended to be higher $(P=0.09)$ for sows with a high vs. low weight loss during lactation (Fig. 8.5).

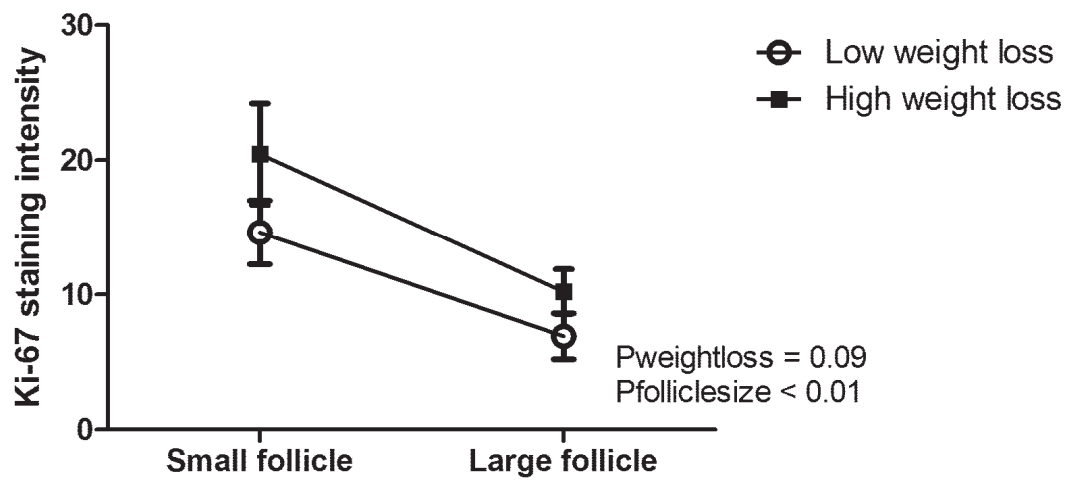

Fig. 8.5 - Ki-67 protein expression in granulosa cells of the smallest and largest follicle of the 10 largest healthy follicles at weaning in sows with high weight loss $(12.0 \pm 2.2 \%, \mathrm{~N}=4)$ and low weight loss $(4.7 \pm 1.5 \%, \mathrm{~N}=4)$ during a 26 -day lactation. To determine protein expression, the mean staining intensity (sum of gray values in all pixels divided by the number of pixels selected) was measured in all visually intact parts of the granulosa cell layer (see Chapter $\mathbf{3}$ for experimental details). Data were analysed using a repeated measures ANOVA in Graphpad Prism (version 5.04, GraphPad Software, La Jolla, CA, USA). The interaction between follicle size and weight loss was not significant. 
To summarize the results of the transcriptome analysis, sows with high relative weight loss during lactation have a higher expression of genes involved in protein translation and some genes involved in proliferation as compared to sows with a low relative weight loss. In addition, at the protein level, granulosa cell proliferation tended to be higher in sows with a higher weight loss during lactation. These results corroborate findings in Chapter 3, where larger and smaller follicles in the follicle pool of the 10 largest healthy follicles were compared for sows with variable weight loss. Smaller follicles were more proliferative, as shown by a higher expression of genes involved in cell cycle progression and protein translation, while larger follicles were more differentiated as shown by a higher expression of follicular maturation markers. Different from Chapter 3, in the current analysis follicle size was similar in high vs. low weight loss sows, and observed effects are therefore due to lactational weight loss. Higher granulosa cell proliferation and less differentiation in sows with a higher relative weight loss during lactation may indicate that lactational weight loss causes a delay in follicular development. In addition, these results indicate that besides effects of weight loss on follicle size, weight loss also sustains granulosa cell proliferation which may delay granulosa cell differentiation, independent of follicle size.

\subsubsection{Summarizing influence of weight loss during lactation on reproductive outcome parameters and follicular developmental competence}

Lactational weight loss negatively influences litter characteristics of the next litter in modern sows. Lactational weight loss affects follicular growth and developmental competence, which can be seen by e.g. a lower average follicle size, hampered oocyte maturation and a changed follicular fluid composition (IGF1 and steroids). In addition, transcriptome analysis shows many differences in granulosa cell gene expression in sows with high vs. low relative weight loss, irrespective of follicle size. In modern sows, the effect of weight loss on WOI is much smaller as compared to earlier studies. As lactational weight loss clearly still affects litter characteristics, this suggests that these negative effects of lactational weight loss on the reproductive performance in the next cycle may be related with a developmental delay in follicular development. Together, this suggests that negative effects of lactation weight loss on follicular development, already evident at the start of the follicular phase, extends to the time of ovulation, and can explain impairments in reproductive outcome parameters. 


\subsection{Influence of energy mobilization of different substrates during lactation on follicular developmental competence}

\subsubsection{Body weight, backfat depth and muscle depth}

As described previously (paragraph 8.2), sows usually experience weight loss during lactation, which negatively influences reproductive performance and the next litter if it exceeds around 10-12\% of initial body weight (Schenkel et al., 2010; Thaker and Bilkei, 2005 and paragraph $\underline{8.2}$ in this discussion, Table 8.6). This lactational weight loss is due to mobilization of adipose tissue and skeletal muscle. Adipose tissue is designed to store energy as triglycerides in their lipid droplets in periods of energy excess. Energy is released form adipose tissue as free fatty acids in time of energy need (reviewed by Luo and Liu, 2016). Skeletal muscle is the largest tissue in the body. During a negative energy balance, skeletal muscle loss can be sustained to some degree. Muscle proteins can break down to amino acids that can be used for energy or milk protein (reviewed by Carbone et al., 2012). During lactation in sows, energy is mobilized from adipose tissue, but also skeletal muscle protein is mobilized as an energy and protein source (e.g. Clowes et al., 2005; Kim and Easter, 2001; Schenkel et al., 2010). It is estimated that sows lose around 5 -fold more kilograms of fat during lactation compared to protein (corrected for the associated water loss) (Bergsma et al., 2009). Elucidating the influence of energy mobilization of different substrates during lactation on follicular developmental competence in modern sows is important, as it could lead to new insights of how follicular developmental competence is affected by energy metabolism. In addition, modern sows have a high genetic merit for leanness which may have consequences for energy mobilization during lactation and its effects on follicular developmental competence.

As both fat and protein stores may be simultaneously used for energy mobilization it is difficult to establish which of the two is responsible for the negative effects of weight loss during lactation on follicular developmental competence. So far, two studies in sows have assessed effects of selective protein mobilization during lactation on follicular developmental competence (Clowes et al., 2003; Yang et al. 2000). In both studies, primiparous sows were fed different amounts of protein during lactation using isocaloric lactation diets. Lower protein levels resulted in a higher estimated protein loss (based on body weight and backfat depth), while backfat depth (as a proxy for adipose tissue stores) remained similar. Selective high protein losses reduced follicle size and follicular fluid $17 \beta$-estradiol levels at weaning (Clowes et al., 2003) and at pro-oestrus (Yang et al., 2000). In addition, follicular fluid obtained at prooestrus from sows fed a low protein diet was less competent to mature oocytes in vitro (Yang et al., 2000). From these studies, it is clear that selective protein mobilization negatively affects follicular development competence. Studies investigating effects of selective energy mobilization from adipose tissue on follicular developmental competence in sows are lacking. In this thesis, loin muscle depth loss during lactation was negatively related to follicle size in the primiparous sows at the mid-follicular phase (Chapter 6) and was unfortunately not 
measured in the multiparous sows at the onset of the follicular phase. Backfat loss was negatively related to follicle size in the primiparous sows (Chapter 6), while it was positively related to follicle size in the multiparous sows (Chapter 2 ). In the latter group of sows, more backfat loss was also related to lower serum creatinine levels (Chapter 2), a marker for whole body protein breakdown during a negative energy balance (Yang et al., 2009). This suggests (as discussed in Chapter 2), that increased energy mobilisation from adipose tissues during lactation could spare protein reserves and thereby improve follicular development. In the primiparous sows, no relation between backfat loss during lactation and muscle depth loss or serum creatinine levels were found (Chapter 7), indicating that increased backfat loss did not have a protein sparing effect in the primiparous sows. This difference might be explained by the amount of available adipose tissue for energy mobilization during lactation, as it has been suggested that sows maintain a certain minimum level of adiposity (Bergsma et al., 2009; Parmley et al., 1996; Whittemore and Morgan, 1990). The essential role of adipose tissue for survival and reproduction is for instance shown by studies in transgenic A-ZIP/F-1 mice which lack white adipose tissue. These mice have a reduced fecundity, a shorter lifespan and are diabetic; the latter can be reversed by surgical implantation of adipose tissue (Moitra et al., 1998; Gavrilova et al., 2000). For sows, the minimum level of adiposity that is maintained is suggested to be at a backfat thickness of around $10 \mathrm{~mm}$ (Whittemore and Morgan, 1990). Indeed, backfat depth at parutrition differed between the mulitparous and primiparous sows, as the multiparous $\mathrm{N}$-line sows had an average backfat depth of $17.1 \pm 0.4 \mathrm{~mm}$ (Chapter 2), while the primiparous TN70 sows had an average backfat depth of $13.7 \pm 0.4 \mathrm{~mm}$ (Chapter 6). This may indicate that the primiparous sows do not have sufficient fat reserves for energy mobilization to spare protein reserves, as their backfat depth is already close to the minimum level of adiposity that is maintained during lactation. In both studies, backfat at parturition was positively related to backfat loss during lactation. Thus, the amount of backfat at parturition seems to determine if backfat loss during lactation will be sufficient to spare protein reserves during lactation. More backfat at parturition and (consequently) less lean mass mobilization during lactation may therefore positively influence follicular developmental competence in highly prolific sows.

\subsubsection{Metabolic parameters in the blood}

Availability and mobilization of energy stores is communicated on a whole-body level. In this thesis, the amount of backfat depth at parturition and at weaning was related to leptin levels at the corresponding time-points (Chapter 6). More weight loss during lactation was negatively related to IGF1 levels (Chapters 2, 6, 7), while backfat depth loss during lactation was related to higher NEFA levels at weaning (Chapter 2 ), and loin muscle depth loss was positively related to creatinine levels at weaning (Chapters 6 and 7). Some of these metabolic factors in the blood were related to follicular characteristics. Studying these relations could 
further increase our understanding of the influence of energy mobilization from different tissues on follicular developmental competence.

IGF1 was positively related to percentage healthy COCs of the 15 largest follicles, both at the onset of the follicular phase and at the mid-follicular phase (Chapter $\mathbf{2}$ and $\mathbf{6}$ ). Higher leptin and IGF1 levels (Chapter $\mathbf{6}$ ) and lower creatinine levels in the blood at weaning were positively related to average follicle size (Chapter $\mathbf{2}$ and 6). Leptin and IGF1 may influence follicular development directly at the ovarian level or via influencing gonadotropin release. The hypothalamus and pituitary express IGF1 and leptin receptors; IGF1 and leptin may therefore play a role in the regulation of the secretion of the gonadotropins FSH and LH (reviewed by Wolfe et al., 2014; Odle et al., 2018). In addition, both peripheral leptin and IGF1 levels influence steroidogenesis and follicular development directly at the ovarian level (e.g. Agarwal et al., 1999; Ruiz-Cortés et al., 2000 and 2003; Xia et al., 1994) by binding to their receptors on granulosa cells and theca cells (Ruiz-Cortés et al., 2000; Spicer and Echternkamp, 1995). In sows, peripheral leptin levels decrease during lactation (Prunier et al., 2001; Chapter 6) and as shown for the first time in this thesis, leptin levels at weaning were positively related to average follicle size in sows in the first half of the follicular phase. Relations between plasma leptin levels and follicular fluid or serum steroid levels could not be detected in the studies described in this thesis, so it is not clear if leptin influences steroidogenesis in early stages of the follicular phase in sows. Direct effects of creatinine on gonadotropin release or on the ovary are unknown. It is therefore possible that the relation between creatinine and average follicle size is indirect and follicle size is in fact regulated by other factors associated with higher lean mass mobilization during lactation. Together, described relations between metabolic parameters in blood and follicular development confirm our previously established relations between backfat loss and muscle depth loss and follicular characteristics. A higher availability of adipose tissue for energy mobilization during lactation and reduced lean mass mobilization may positively influence follicular growth around weaning and may thereby improve post-weaning reproductive parameters.

Next to the previously mentioned metabolic parameters, other parameters may also play a role. Next to leptin, white adipocytes also signal via the production of other adipokines which are released in the blood, such as adiponectin, resistin, chemerin and retinol binding protein 4 (RBP4) (reviewed by Mathew et al., 2018). The most abundant plasma adipokine is adiponectin, and its expression decreases with increasing adiposity. Adiponectin plays a key role in controlling energy metabolism, but also regulates follicular development (reviewed by Barbe et al., 2019). Adiponectin receptors (AdipoR1 and AdipoR2) are expressed in the hypothalamus and pituitary, where its activation can play a role in the regulation of FSH and LH secretion. In addition, AdipoR1 and AdipoR2 are expressed by the oocyte, granulosa cells and theca cells, where its activation at least in the granulosa and theca cells regulates steroidogenesis in for instance humans and bovine (Chabrolle et al., 2009; Lagaly et al., 2008). 
Furthermore, addition of adiponectin to oocyte maturation medium increased meiotic maturation of oocytes from large follicles (>6 mm) in pigs (Chappaz et al., 2008). It would therefore be interesting to further study the role of adipokines such as adiponectin as metabolic signalling factors in regulating follicular developmental competence. Similar to adipose tissue, skeletal muscle secretes myokines (e.g. IL-6, IL-15, brain-derived neurotrophic factor) which regulate energy metabolism on a whole-body level (reviewed by Pedersen, 2011). One of these myokines (which is also a hepatokine as it is also produced by the liver), is fibroblast growth factor 21 (FGF21), a metabolic regulator of glucose and lipid metabolism (reviewed by: Fisher and Maratos-Flier, 2016 and Tezze et al., 2019). FGF21 signals nutrient restriction, as FGF21 levels are higher in mice and rats which were fed an iso-caloric lowprotein diet (Laeger et al., 2014), and are higher in dairy cows during lactation (Schoenberg et al., 2011), which experience a NEB. Studies using mice show, via exposure of mice to FGF21, that FGF21 may supress ovulation via acting on the SCN in the hypothalamus (Owen et al., 2013). The role of many of these signalling factors, e.g. adipokines and myokines, in influencing follicular developmental competence of sows remain to be elucidated. Studying these adipokines and myokines, as an addition to the metabolic factors that have already been measured in this thesis, may help to increase our understanding of the influence of energy mobilization from different tissues on follicular developmental competence.

\subsubsection{Summarizing influence of energy mobilization of different substrates during lactation on follicular developmental competence}

Multiparous sows with more backfat loss, and therefore higher energy mobilization from adipose tissues, have less lean mass mobilization during lactation as compared to sows with less backfat loss. In addition, more backfat depth loss was associated with a larger average follicle size. This effect was not seen in primiparous sows, which might be due to the fact that the primiparous sows had less body fat reserves at the start of lactation as compared to multiparous sows. The amount of backfat of sows at parturition may determine if energy mobilization from backfat during lactation can provide sufficient energy for milk production to spare protein reserves during lactation. This has consequences for follicular developmental competence, as especially lean mass mobilization in sows with low adipose tissue reserves seems detrimental for follicular development. 


\subsection{Implications of this research and recommendations for future research}

\subsubsection{Implications for current sow management strategies}

As a response to the demand of consumers for leaner pork, modern sows have been selected for a higher capacity for lean mass gain, and a lower capacity for fat mass gain. Next to this, modern sows have also been selected for a large litter size to increase pig production profitability. As a consequence, sows need to produce a high amount of milk to feed the higher number of piglets weaned per litter (Kemp et al., 2018). This high milk production leads to high metabolic demands of sows during lactation. Feed intake during lactation is not sufficient to meet these high metabolic demands, which is especially true for modern sows and more so for primiparous sows which have a lower feed intake capacity (Kanis, 1990; Bergsma et al., 2009). Therefore, to meet the high metabolic demands during lactation, sows need to mobilise their own body reserves. Together, these described breeding strategies have resulted in lean sows with a low fatness, which mobilise a large amount of body reserves during lactation. From this thesis it became clear that a certain level of adiposity is needed for energy mobilization during lactation to reduce mobilization of lean mass, otherwise follicular developmental competence will be compromised (Chapter 2, 6). This compromised follicular developmental competence is expected to negatively affect reproductive performance in the next cycle. On the other hand, more mobilization of tissue for energy during lactation was related to a higher milk production (Chapter $\mathbf{7}$ ). More mobilization of energy and protein from lean mass was specifically related to more milk protein production. This implies that especially moiblization of substrates from lean mass may be beneficial to support piglet weight gain, as otherwise the amount of milk protein production may be insufficient for maximum piglet gain (Campbell and Dunkin, 1983). Both aspects, milk production to support litter weight gain of the current litter and follicular development of the presumptive ovulatory follicle pool which is expected to affect litter characteristics of the next litter, should be optimized for efficient pig production.

One of the strategies to improve efficiency may be to increase body fat reserves of sows before the start of lactation. This may be achieved by using older gilts with more body tissue reserves at insemination or by increasing the level or composition of gestation feeds. However, heavier and fatter sows at parturition also have a reduced voluntary feed intake during lactation (reviewed by Eissen et al., 2000; Quesnel et al., 2005) which may result in increased body reserve mobilization during lactation and thereby negatively affect follicular developmental competence. This effect can also be seen in Table 8.6 (paragraph 8.2.1), which shows that primiparous sows with the highest lactational weight loss ( $>12 \%$ of initial body weight) and a second litter dip in reproductive performance, also had the highest body weight and backfat depth at parturition. Using heavier and fatter sows at gestation may therefore not always be the best strategy to support follicular development during and after lactation, as its 
success may be dependent on factors such as age of the sows and sow feed intake capacity. More research regarding this aspect is needed.

Other possible strategies may be directed towards increasing the availability of energy and nutrients in the lactation diet, to support both follicular development during lactation and milk production. Some relatively recent studies have been directed towards feeding higher protein levels during lactation (Huang et al., 2013; Pedersen et al., 2019; Strathe et al., 2017). In the study of Strathe et al. (2017), gradual increases of dietary protein levels during lactation were applied, which decreased lactational weight loss and increased backfat loss. In addition, the higher protein levels tended to be related to an increased litter size in the next litter (Strathe et al., 2017), which suggests that feeding higher protein levels during lactation improves follicular developmental competence of the ovulatory follicle pool. Next to this, feeding higher levels of protein during lactation increases both milk fat content (Strathe et al., 2017) and milk protein content (Clowes et al., 2003; Strathe et al., 2017). Feeding a higher protein level during lactation may therefore positively influence both milk (protein) production to support litter gain of the current litter and follicular developmental competence to improve litter characteristics of the next litter. However, it remains to be investigated how feeding high protein levels influences follicular developmental competence and how it affects piglet birth weight in the next litter. Improving piglet birth weight and reducing within-litter variation in piglet birth weights would ultimately contribute to reduced piglet mortality and improved piglet welfare. Other studies have used increased energy density in lactation diets using fats or oil. This additional energy was, however, mainly used for the production of milk with a higher fat content, resulting in a higher litter gain (Rosero et al., 2015; reviewed by Rosero et al., 2016), and did not benefit sow body condition. Similarly, feeding sows a fat-rich versus starch-rich iso-caloric lactation diet, resulted in more milk fat production and fatter piglets, but also resulted in a more negative energy balance when sows were fed at a high feeding level (van den Brand et al., 2000). This suggests that adding fat to the lactation diet mainly results in increased milk fat production, which is beneficial for litter gain, but does not increase energy availability for follicular development during lactation. In conclusion, it remains a challenge to design optimal feeding strategies for young lactating sows with a relatively short lactation length, as strategies that benefit the current litter (sustained milk production) seem to be a disadvantage for the next generation (due to suboptimal follicle development).

\subsubsection{Recommendations for future research}

Firstly, in this thesis, several physiological and molecular characteristics are associated with follicular developmental competence of the follicles that are expected to ovulate. This was done by comparing larger and smaller follicles within the follicle pool, by comparing follicles of sows with a high or low average follicle size and by comparing follicles of sows with a high or low percentage of $\mathrm{COC}$ health. Follicular developmental competence was mainly 
characterized by the degree of granulosa cell differentiation vs. proliferation, steroidogenesis and granulosa cell IGF1 production. The use of other models with distinct differences in follicular developmental competence, such as maternal aging or the comparison of adult sows to pre-pubertal gilts, may further confirm or identify new follicular characteristics associated with follicular developmental competence. In this thesis only mural granulosa cells and follicular fluid were analysed. For future studies, it would be interesting to study molecular characteristics of the oocyte and other follicular somatic cells (e.g. cumulus cells and theca cells) in relation to markers of follicular competence. This is important as these different follicular cell types produce paracrine growth factors and steroids (somatic cells only) to communicate with each other and to regulate follicular developmental competence. In addition, studies on a single follicle level, would contribute knowledge of the complex interplay between these different follicular cell types in relation to follicular developmental competence. To further confirm the relevance of identified follicular characteristics for the developmental competence of the ovulatory follicle pool, it would be interesting to also study these characteristics in later stages of the follicular phase. In this thesis, it was argued that follicular developmental competence in sows, already from the onset of the follicular phase onwards, may determine oocyte quality, embryo development and finally piglet birth weight and survival. Directing future research towards investigation of molecular mechanisms that underlie follicular developmental competence, as was done in this thesis, may further improve our understanding of reproductive problems in sows and other mammals.

Secondly, this thesis resulted in new insights of how the metabolic state of the sow during lactation influences follicular developmental competence. Weight loss affected follicular growth and developmental competence, by reducing follicle size, hampered oocyte maturation and delayed zygote development after IVF. Observed changes in follicular fluid composition (e.g. steroids and IGF1) may partially explain these observed differences in follicular developmental competence. However, further molecular substantiation of potential regulated pathways (e.g. IGF1, EGF or AR signalling) in these follicles would greatly contribute to our understanding of metabolic influences on follicular developmental competence. Results of this thesis suggest that both peripheral and follicular produced IGF1 may play a key role in determining effects of body reserve mobilization on follicular developmental competence. Future studies may be directed at better understanding the role of IGF1 in follicular developmental competence under different physiological conditions. This may for instance be done via continuous IGF1 administration via a subcutaneous pump in sows which experience either high or low weight loss during lactation. As a next step, new feeding strategies may be developed, such as insulin or IGF1 stimulating diets (e.g. Wientjes et al., 2012b) to improve follicular development in sows. Next to studying the influence of changes in the lactational metabolic state on antral follicular development, it would be interesting to study other time-periods as well. For instance gestation, when pre-antral follicular development of the follicles that will become the pre-ovulatory follicle pool is already 
initiated. Nutrition may especially affect oocyte quality at this stage of development, as oocytes only grow during pre-antral follicular development and are transcriptionally silent during antral follicular development. This hypothesis is corroborated by Britt (1992), which describes that dairy cows which lose more body reserves during the first 5 weeks after calving, so during the time pre-antral follicular development of the ovulatory follicle pool takes place, had lower fertility. Studying the influence of metabolism on follicular development in other metabolic states than lactation, when sows usually experience a negative energy balance, will also increase translatability of the findings in sows to other mammals.

Thirdly, results from this thesis show that especially lactational mobilization of energy from lean mass in sows which have low adipose tissue reserves, negatively impacts follicular developmental competence. In this thesis, feed restriction was applied, where sows simultaneously lost both body protein and fat. It would be beneficial to study the effect of selective protein and fat loss during lactation on follicular developmental competence. For selective protein loss, this may be achieved through varying protein levels in the diet. A possible problem of such a study may be that selective fat mass loss may be difficult to achieve in modern sows with low adiposity. As an alternative, assessment of relations between additional metabolic signalling factors of adipose tissue (adipokines) or skeletal muscle (myokines) and follicular characteristics could lead to new insights in how follicular development is regulated by metabolism of different substrates (e.g. fat or protein). Existing studies using other animal models, such as transgenic mice, have already established the role of many of these signalling factors in metabolism. These studies may be used to direct research of metabolic influences on follicular developmental competence towards the most interesting metabolic signalling factors for further analysis. If relations between metabolism and follicular developmental competence are better understood, future studies could be directed towards studying potential beneficial effects of changes in metabolic state at the start of lactation or redirecting energy mobilization during lactation. This may optimize follicular developmental competence after weaning and can potentially prevent reproductive problems in sows and other mammals.

\subsection{General conclusions}

This thesis investigated molecular physiological characteristics of follicular developmental competence and how these characteristics are influenced by the metabolic state of sows. This was done in order to test our hypothesis that impairments in development of antral follicles may partially explain reproductive problems in highly prolific sows.

Overall, the observations as outlined in this thesis, yield the following general conclusions: 


\section{Characteristics of follicular developmental competence}

1: There is a high variability in both average follicle size and COC health between sows in the same early stage of the oestrus cycle after lactation, but there is also high variability within the follicle pool of follicles which are expected to ovulate.

2: Larger follicles in the presumptive ovulatory follicle pool are characterized by higher granulosa cell differentiation and less proliferation, suggesting a developmental delay of the smaller follicles. This variability in the follicle pool and granulosa cells, may have consequences for (variation in) oocyte quality, and eventual embryo and foetal survival and development.

3: Follicular fluid steroid levels reflect average follicle size and average COC health of the follicle pool, while steroid levels in serum are generally low and poorly reflect the developmental stage in the first half of the follicular phase of the oestrus cycle.

4: Sows can be used as an animal model to study follicular development as it provides several advantages over other animal models; 1 ) sows are poly-ovulatory which allows for the study of differences in follicular characteristics within the follicle pool 2) sows have a well-defined start of the follicular phase at weaning.

Influence of the metabolic state of the sows

5: In modern prolific primiparous sows, a high lactational weight loss during the first lactation negatively influences reproductive performance and litter characteristics of the second litter. The threshold in weight loss for reduced reproductive performance is similar to what has been found for older, less prolific sow lines. Next to this, lactational weight loss due to feed restriction highly influences follicular developmental competence already at the first half of the follicular phase. This provides further evidence for our hypothesis that impairments in follicular developmental competence may partially explain a lower reproductive performance in the next cycle.

6: Both peripheral and follicular produced IGF1 may play a key role in determining effects of body reserve mobilization on follicular developmental competence.

7: The degree and type of body reserve mobilization i.e. fat or protein, during lactation is highly determined by the metabolic state of the sows at parturition. In addition, it seems that especially energy mobilization from lean mass in sows with low adipose tissue reserves, negatively impacts follicular developmental competence. Altering either the metabolic state of the sow at parturition or directing energy mobilization during lactation may improve reproductive performance in sows.

8: The degree and type of body reserve mobilization during lactation affects milk production and milk composition, which influences piglet growth and performance. 


\section{References}

Agarwal, S. K., Vogel, K., Weitsman, S. R., \& Magoffin, D. A. (1999). Leptin antagonizes the insulin-like growth factor-I augmentation of steroidogenesis in granulosa and theca cells of the human ovary. The journal of clinical endocrinology \& metabolism, 84(3), 1072-1076.

Alvarez, G. M., Dalvit, G. C., Achi, M. V., Miguez, M. S., \& Cetica, P. D. (2009). Immature oocyte quality and maturational competence of porcine cumulus-oocyte complexes subpopulations. Biocell, 33(3), 167-177.

Amdi, C., Giblin, L., Hennessy, A. A., Ryan, T., Stanton, C., Stickland, N. C., \& Lawlor, P. G. (2013). Feed allowance and maternal backfat levels during gestation influence maternal cortisol levels, milk fat composition and offspring growth. Journal of nutritional science 2, e1.

Baidoo, S., Aherne, F., Kirkwood, R., \& Foxcroft, G. (1992). Effect of feed intake during lactation and after weaning on sow reproductive performance. Canadian journal of animal science, 72(4), 911-917.

Barbe, A., Bongrani, A., Mellouk, N., Estienne, A., Kurowska, P., Grandhaye, J., Elfassy, Y., Levy, R., Rak, A., \& Froment, P. (2019). Mechanisms of adiponectin action in fertility: An overview from gametogenesis to gestation in humans and animal models in normal and pathological conditions. International journal of molecular sciences 20(7), 1526.

Bergsma, R., Kanis, E., Verstegen, M., van der Peet-Schwering, C., \& Knol, E. (2009). Lactation efficiency as a result of body composition dynamics and feed intake in sows. Livestock science, 125(2), 208-222.

Britt, J. (1992). Impacts of early postpartum metabolism on follicular development and fertility. The Bovine Practitioner Proceedings 24, 39-43.

Brüssow, K. P., Ratky, J., \& Rodriguez-Martinez, H. (2008). Fertilization and early embryonic development in the porcine fallopian tube. Reproduction in domestic animals. Supplement 43(2), 245-251.

Campbell, R., \& Dunkin, A. (1983). The influence of protein nutrition in early life on growth and development of the pig: 1 . Effects on growth performance and body composition. British journal of nutrition, 50(3), 605-617.

Chabrolle, C., Tosca, L., Ramé, C., Lecomte, P., Royère, D., \& Dupont, J. (2009). Adiponectin increases insulin-like growth factor I-induced progesterone and estradiol secretion in human granulosa cells. Fertility and sterility 92(6), 1988-1996.

Chappaz, E., Albornoz, M. S., Campos, D., Che, L., Palin, M.-F., Murphy, B. D., \& Bordignon, V. (2008). Adiponectin enhances in vitro development of swine embryos. Domestic animal endocrinology 35(2), 198-207.

Chen, Z., \& Dziuk, P. (1993). Influence of initial length of uterus per embryo and gestation stage on prenatal survival, development, and sex ratio in the pig. Journal of animal science, 71(7), 18951901.

Chun, S.-Y., Billig, H., Tilly, J., Furuta, I., Tsafriri, A., \& Hsueh, A. (1994). Gonadotropin suppression of apoptosis in cultured preovulatory follicles: mediatory role of endogenous insulin-like growth factor I. Endocrinology, 135(5), 1845-1853.

Clowes, E., Aherne, F., Foxcroft, G., \& Baracos, V. (2003). Selective protein loss in lactating sows is associated with reduced litter growth and ovarian function. Journal of animal science, 81(3), 753-764.

Clowes, E. J., Aherne, F. X., \& Baracos, V. E. (2005). Skeletal muscle protein mobilization during the progression of lactation. American journal of physiology-endocrinology and metabolism 288, E564-E572.

Cohen, P. A., Donini, C. F., Nguyen, N. T., Lincet, H., \& Vendrell, J. A. (2015). The dark side of ZNF217, a key regulator of tumorigenesis with powerful biomarker value. Oncotarget, 6(39), 41566.

Colledge, M., \& Scott, J. D. (1999). AKAPs: from structure to function. Trends in cell biology, 9(6), 216221. 
Da Silva, C. L. A., Broekhuijse, M., Laurenssen, B., Mulder, H., Knol, E. F., Kemp, B., \& Soede, N. M. (2017). Relationship between ovulation rate and embryonic characteristics in gilts at $35 \mathrm{~d}$ of pregnancy. Journal of animal science, 95(7), 3160-3172.

De Bettio, S., Maiorka, A., Barrilli, L., Bergsma, R., \& Silva, B. (2016). Impact of feed restriction on the performance of highly prolific lactating sows and its effect on the subsequent lactation. Animal 10(3), 396-402.

De Vos, A., Van De Velde, H., Joris, H., Verheyen, G., Devroey, P., \& Van Steirteghem, A. (2003). Influence of individual sperm morphology on fertilization, embryo morphology, and pregnancy outcome of intracytoplasmic sperm injection. Fertility and sterility, 79(1), 42-48.

Einarsson, S., Brandt, Y., Lundeheim, N., \& Madej, A. (2008). Stress and its influence on reproduction in pigs: a review. Acta veterinaria scandinavica $50,48$.

Eissen, J., Kanis, E., \& Kemp, B. (2000). Sow factors affecting voluntary feed intake during lactation. Livestock production science 64(2-3), 147-165.

Faraj, N., Alhalabi, M., \& Al-Quobaili, F. (2017). Predictive value of follicular fluid insulin like growth factor-1 in IVF outcome of normo-ovulatory women. Middle east fertility society journal, 22(2), 101-104.

Fisher, F. M., \& Maratos-Flier, E. (2016). Understanding the Physiology of FGF21. Annual review of physiology 78, 223-241.

Ford, S., Vonnahme, K., \& Wilson, M. (2002). Uterine capacity in the pig reflects a combination of uterine environment and conceptus genotype effects. Journal of animal science. Supplement, 80, 66-73.

Fowden, A., Sferruzzi-Perri, A., Coan, P., Constancia, M., \& Burton, G. (2009). Placental efficiency and adaptation: endocrine regulation. The journal of physiology, 587(14), 3459-3472.

Gavrilova, O., Marcus-Samuels, B., Graham, D., Kim, J. K., Shulman, G. I., Castle, A. L., Vinson, C., Eckhaus, M., \& Reitman, M. L. (2000). Surgical implantation of adipose tissue reverses diabetes in lipoatrophic mice. The journal of clinical investigation 105(3), 271-278.

Geisert, R. D., Brookbank, J. W., Michael Roberts, R., \& Bazer, F. W. (1982). Establishment of pregnancy in the pig: II. Cellular remodeling of the porcine blastocyst during elongation on day 12 of pregnancy. Biology of reproduction, 27(4), 941-955.

Gerdes, J., Lemke, H., Baisch, H., Wacker, H.-H., Schwab, U., \& Stein, H. (1984). Cell cycle analysis of a cell proliferation-associated human nuclear antigen defined by the monoclonal antibody Ki67. The journal of immunology, 133(4), 1710-1715.

Grupen, C. G., \& Armstrong, D. T. (2010). Relationship between cumulus cell apoptosis, progesterone production and porcine oocyte developmental competence: temporal effects of follicular fluid during IVM. Reproduction, fertility and development, 22(7), 1100-1109.

Guthrie, H., \& Cooper, B. (1996). Follicular atresia, follicular fluid hormones, and circulating hormones during the midluteal phase of the estrous cycle in pigs. Biology of reproduction, 55(3), 543-547.

Guthrie, H., Garrett, W., \& Cooper, B. (1998). Follicle-stimulating hormone and insulin-like growth factor-I attenuate apoptosis in cultured porcine granulosa cells. Biology of reproduction, 58(2), 390-396.

Hayashi, K.-G., Acosta, T. J., Tetsuka, M., Berisha, B., Matsui, M., Schams, D., Ohtani, M., \& Miyamoto, A. (2003). Involvement of angiopoietin-tie system in bovine follicular development and atresia: messenger RNA expression in theca interna and effect on steroid secretion. Biology of reproduction 69(6), 2078-2084.

Hazzard, T. M., Molskness, T. A., Chaffin, C. L., \& Stouffer, R. L. (1999). Vascular endothelial growth factor (VEGF) and angiopoietin regulation by gonadotrophin and steroids in macaque granulosa cells during the peri-ovulatory interval. MHR: Basic science of reproductive medicine 5I(12), 1115-1121.

Hickey, T. E., Marrocco, D. L., Amato, F., Ritter, L. J., Norman, R. J., Gilchrist, R. B., \& Armstrong, D. T. (2005). Androgens augment the mitogenic effects of oocyte-secreted factors and growth differentiation factor 9 on porcine granulosa cells. Biology of reproduction, 73(4), 825-832. 
Hirshfield, A. N. \& Midgley, A. R. (1978). The role of FSH in the selection of large ovarian follicles in the rat. Biology of reproduction, 19(3), 606-611.

Hoving, L., Soede, N., Graat, E., Feitsma, H., \& Kemp, B. (2010). Effect of live weight development and reproduction in first parity on reproductive performance of second parity sows. Animal reproduction science 122, 82-89.

Hoving, L., Soede, N., Feitsma, H., \& Kemp, B. (2012). Lactation weight loss in primiparous sows: consequences for embryo survival and progesterone and relations with metabolic profiles. Reproduction in domestic animals, 47(6), 1009-1016.

Hu, Z.-Y., Liu, Y.-P., Xie, L.-Y., Wang, X.-Y., Yang, F., Chen, S.-Y., \& Li, Z.-G. (2016). AKAP-9 promotes colorectal cancer development by regulating $\mathrm{Cdc} 42$ interacting protein 4 . Biochimica et biophysica acta (BBA)-molecular basis of disease, 1862(6), 1172-1181.

Huang, F., Liu, H., Sun, H., \& Peng, J. (2013). Effects of lysine and protein intake over two consecutive lactations on lactation and subsequent reproductive performance in multiparous sows. Livestock science, 157(2-3), 482-489.

Hunter, M., \& Wiesak, T. (1990). Evidence for and implications of follicular heterogeneity in pigs. Journal of reproduction and fertility. Supplement, 40, 163-177.

Jiang, J.-Y., Xiong, H., Cao, M., Xia, X., Sirard, M.-A., \& Tsang, B. K. (2010). Mural granulosa cell gene expression associated with oocyte developmental competence. Journal of ovarian research, $3(1), 6$.

Kauffold, J., Gottschalk, J., Schneider, F., Beynon, N., \& Wähner, M. (2008). Effects of feeding level during lactation on FSH and $\mathrm{LH}$ secretion patterns, and follicular development in primiparous sows. Reproduction in domestic animals, 43(2), 234-238.

Kanis, E. (1990). Effect of food intake capacity on production traits in growing pigs with restricted feeding. Animal science 50, 333-341.

Kelly, C., Socha, T., \& Zimmerman, D. R. (1988). Characterization of gonadotropic and ovarian steroid hormones during the periovulatory period in high ovulating select and control line gilts. Journal of animal science, 66(6), 1462-1474.

Kemp, B., Da Silva, C, \& Soede, N. M. (2018). Recent advances in pig reproduction: Focus on impact of genetic selection for female fertility. Reproduction in domestic animals, 53, 28-36.

Keryer, G., Witczak, O., Delouvée, A., Kemmner, W. A., Rouillard, D., Taskén, K., \& Bornens, M. (2003). Dissociating the centrosomal matrix protein AKAP450 from centrioles impairs centriole duplication and cell cycle progression. Molecular biology of the cell, 14(6), 2436-2446.

Kim, S., \& Easter, R. (2001). Nutrient mobilization from body tissues as influenced by litter size in lactating sows. Journal of animal science 79, 2179-2186.

King, R. H., \& Williams, I. H. (1984). The effect of nutrition on the reproductive performance of firstlitter sows 1 . Feeding level during lactation, and between weaning and mating. Animal science, 38(02), 241-247.

Kirkwood, R., Baidoo, S., Aherne, F., \& Sather, A. (1987). The influence of feeding level during lactation on the occurrence and endocrinology of the postweaning estrus in sows. Canadian journal of animal science, 67(2), 405-415.

Kirkwood, R., Baidoo, S., \& Aherne, F. (1990). The influence of feeding level during lactation and gestation on the endocrine status and reproductive performance of second parity sows. Canadian journal of animal science, 70(4), 1119-1126.

Knox, R., Vatzias, G., Naber, C., \& Zimmerman, D. (2003). Plasma gonadotropins and ovarian hormones during the estrous cycle in high compared to low ovulation rate gilts. Journal of animal science, 81(1), 249-260.

Krig, S. R., Jin, V. X., Bieda, M. C., O'Geen, H., Yaswen, P., Green, R., \& Farnham, P. J. (2007). Identification of genes directly regulated by the oncogene ZNF217 using chromatin immunoprecipitation (ChIP)-chip assays. Journal of biological chemistry, 282(13), 9703-9712.

Laeger, T., Henagan, T. M., Albarado, D. C., Redman, L. M., Bray, G. A., Noland, R. C., Münzberg, H., Hutson, S. M., Gettys, T. W., \& Schwartz, M. W. (2014). FGF21 is an endocrine signal of protein restriction. The journal of clinical investigation 124(9), 3913-3922. 
Lagaly, D. V., Aad, P. Y., Grado-Ahuir, J. A., Hulsey, L. B., \& Spicer, L. J. (2008). Role of adiponectin in regulating ovarian theca and granulosa cell function. Molecular and cellular endocrinology 284(1-2), 38-45.

Losada, A., Yokochi, T., \& Hirano, T. (2005). Functional contribution of Pds5 to cohesin-mediated cohesion in human cells and Xenopus egg extracts. Journal of cell science, 118(10), 2133-2141.

Luo, L., \& Liu, M. (2016). Adipose tissue in control of metabolism. Journal of endocrinology 231(3), R77R99.

Mannini, L., Menga, S., \& Musio, A. (2010). The expanding universe of cohesin functions: a new genome stability caretaker involved in human disease and cancer. Human mutation 31(6), 623-630.

Matsuda, F., Inoue, N., Manabe, N., \& Ohkura, S. (2012). Follicular growth and atresia in mammalian ovaries: regulation by survival and death of granulosa cells. Journal of reproduction and development, 58(1), 44-50.

Mathew, H., Castracane, V. D., \& Mantzoros, C. (2018). Adipose tissue and reproductive health. Metabolism 86, 18-32.

May, J. V., \& Schomberg, D.W. (1984). Developmental coordination of luteinizing hormone/human chorionic gonadotropin (hCG) receptors and acute hCG responsiveness in cultured and freshly harvested porcine granulosa cells. Endocrinology 114(1), 153-163.

Metzger, E., Willmann, D., Mcmillan, J., Forne, I., Metzger, P., Gerhardt, S., Petroll, K., Von Maessenhausen, A., Urban, S., \& Schott, A.-K. (2016). Assembly of methylated KDM1A and CHD1 drives androgen receptor-dependent transcription and translocation. Nature structural \& molecular biology 23(2), 132-139.

Mikkelsen, A. L., \& Lindenberg, S. (2001). Morphology of in-vitro matured oocytes: impact on fertility potential and embryo quality. Human reproduction, 16(8), 1714-1718.

Moitra, J., Mason, M. M., Olive, M., Krylov, D., Gavrilova, O., Marcus-Samuels, B., Feigenbaum, L., Lee, E., Aoyama, T., \& Eckhaus, M. (1998). Life without white fat: a transgenic mouse. Genes \& development 12, 3168-3181.

Nagano, M., Katagiri, S., \& Takahashi, Y. (2006). Relationship between bovine oocyte morphology and in vitro developmental potential. Zygote, 14(1), 53-61.

Odle, A. K., Akhter, N., Syed, M. M., Allensworth-James, M. L., Beneš, H., Melgar Castillo, A. I., MacNicol, M. C., MacNicol, A. M., \& Childs, G. V. (2018). Leptin regulation of Gonadotrope Gonadotropinreleasing Hormone receptors as a Metabolic Checkpoint and Gateway to reproductive Competence. Frontiers in endocrinology, 8, 367.

Oktem, O., \& Urman, B. (2010). Understanding follicle growth in vivo. Human reproduction, 25(12), 2944-2954.

Owen, B. M., Bookout, A. L., Ding, X., Lin, V. Y., Atkin, S. D., Gautron, L., Kliewer, S. A., \& Mangelsdorf, D. J. (2013). FGF21 contributes to neuroendocrine control of female reproduction. Nature medicine (19)(9), 1153-1156.

Parmley, K. L., Machado, C. R., \& McNamara, J. P. (1996). Rates of lipid metabolism in adipose tissue of pigs adapt to lactational state and dietary energy restriction. The journal of nutrition, 126(6), 1644-1656.

Patterson, J., Smit, M., Novak, S., Wellen, A., \& Foxcroft, G. (2011). Restricted feed intake in lactating primiparous sows. I. Effects on sow metabolic state and subsequent reproductive performance. Reproduction, fertility and development, 23(7), 889-898.

Pedersen, B. K. (2011). Muscles and their myokines. Journal of experimental Biology 214(2), 337-346.

Pedersen, T. F., Bruun, T. S., Trottier, N. L., \& Theil, P. K. (2019). Nitrogen utilization of lactating sows fed increasing dietary protein. Journal of animal science, 97(8), 3472-3486.

Peters, J. M. (2012). The many functions of cohesin-different rings to rule them all? The EMBO journal, 31(9), 2061-2063.

Pope, W., \& First, N. (1985). Factors affecting the survival of pig embryos. Theriogenology 23(1), 91105. 
Prunier, A., Guadarrama, C. A. M., Mourot, J., \& Quesnel, H. (2001). Influence of feed intake during pregnancy and lactation on fat body reserve mobilisation, plasma leptin and reproductive function of primiparous lactating sows. Reproduction nutrition development, 41(4), 333-347.

Quesnel, H., Pasquier, A., Mounier, A.-M., Louveau, I., \& Prunier, A. (1998). Influence of feed restriction in primiparous lactating sows on body condition and metabolic parameters. Reproduction nutrition development, 38(3), 261-274.

Quesnel, H., Mejia-Guadarrama, C.A., Pasquier, A., Dourmad, J.-Y., \& Prunier, A. (2005). Dietary protein restriction during lactation in primiparous sows with different live weights at farrowing: II. Consequences on reproductive performance and interactions with metabolic status. Reproduction nutrition development 45(1), 57-68.

Quesnel, H., Brossard, L., Valancogne, A., \& Quiniou, N. (2008). Influence of some sow characteristics on within-litter variation of piglet birth weight. Animal, 2(12), 1842-1849.

Quiniou, N., Dagorn, J., \& Gaudré, D. (2002). Variation of piglets' birth weight and consequences on subsequent performance. Livestock production science, 78(1), 63-70.

Robinson, R. S., Woad, K. J., Hammond, A. J., Laird, M., Hunter, M. G., \& Mann, G. E. (2009). Angiogenesis and vascular function in the ovary. Reproduction 138, 869-881.

Rosero, D., Odle, J., Mendoza, S., Boyd, R., Fellner, V., \& Van Heugten, E. (2015). Impact of dietary lipids on sow milk composition and balance of essential fatty acids during lactation in prolific sows. Journal of animal science, 93(6), 2935-2947.

Rosero, D. S., Boyd, R. D., Odle, J., \& van Heugten, E. (2016). Optimizing dietary lipid use to improve essential fatty acid status and reproductive performance of the modern lactating sow: a review. Journal of animal science and biotechnology, 7(1), 34.

Ruiz-Cortés, Z. T., Men, T., Palin, M. F., Downey, B. R., Lacroix, D. A., \& Murphy, B. D. (2000). Porcine leptin receptor: molecular structure and expression in the ovary. Molecular reproduction and development: incorporating gamete research, 56(4), 465-474.

Ruiz-Cortés, Z. T., Martel-Kennes, Y., Gévry, N. Y., Downey, B. R., Palin, M.-F., \& Murphy, B. D. (2003). Biphasic effects of leptin in porcine granulosa cells. Biology of reproduction, 68(3), 789-796.

Russell, D. L., \& Robker, R. L. (2007). Molecular mechanisms of ovulation: co-ordination through the cumulus complex. Human reproduction update 13(3), 289-312.

Rutherford, K., Baxter, E., D'eath, R., Turner, S., Arnott, G., Roehe, R., Ask, B., Sandoe, P. Moustsen, V. A., \& Thorup, F. (2013). The welfare implications of large litter size in the domestic pig I: biological factors. Animal welfare, 22(2), 199-218.

Sasseville, M., Ritter, L. J., Nguyen, T. M., Liu, F., Mottershead, D. G., Russell, D. L., \& Gilchrist, R. B. (2010). Growth differentiation factor 9 signaling requires ERK1/2 activity in mouse granulosa and cumulus cells. Journal of cell science, 123(18), 3166-3176.

Schenkel, A., Bernardi, M., Bortolozzo, F., \& Wentz, I. (2010). Body reserve mobilization during lactation in first parity sows and its effect on second litter size. Livestock science, 132(1-3), 165172.

Schimenti, K. J., Feuer, S. K., Griffin, L. B., Graham, N. R., Bovet, C. A., Hartford, S., Pendola, J., Lessard, C., Schimenti, J. C. \& Ward, J. O. (2013). AKAP9 is essential for spermatogenesis and sertoli cell maturation in mice. Genetics, 194(2), 447-457.

Schoenberg, K. M., Giesy, S. L., Harvatine, K. J., Waldron, M. R., Cheng, C., Kharitonenkov, A., \& Boisclair, Y. R. (2011). Plasma FGF21 is elevated by the intense lipid mobilization of lactation. Endocrinology 152(12), 4652-4661.

Shaw, H., \& Foxcroft, G. (1985). Relationships between LH, FSH and prolactin secretion and reproductive activity in the weaned sow. Journal of reproduction and fertility, 75(1), 17-28.

Simon, L., Murphy, K., Shamsi, M., Liu, L., Emery, B., Aston, K., Hotaling, J. \& Carrell, D. (2014). Paternal influence of sperm DNA integrity on early embryonic development. Human reproduction, 29(11), 2402-2412.

Smith, O., \& Akinbamijo, O. (2000). Micronutrients and reproduction in farm animals. Animal reproduction science 60-61, 549-560. 
Spicer, L. J., \& Echternkamp, S. E. (1995). The ovarian insulin and insulin-like growth factor system with an emphasis on domestic animals. Domestic animal endocrinology, 12(3), 223-245.

Spötter, A., \& Distl, O. (2006). Genetic approaches to the improvement of fertility traits in the pig. The veterinary journal, 172(2), 234-247.

Strathe, A. V., Bruun, T. S., Geertsen, N., Zerrahn, J.-E., \& Hansen, C. F. (2017). Increased dietary protein levels during lactation improved sow and litter performance. Animal feed science and technology 232, 169-181.

Sugiura, K., Pendola, F. L., \& Eppig, J. J. (2005). Oocyte control of metabolic cooperativity between oocytes and companion granulosa cells: energy metabolism. Developmental biology, 279(1), 20-30.

Summers, A. F., Pohlmeier, W. E., Sargent, K. M., Cole, B. D., Vinton, R. J., Kurz, S. G., Mcfee, R. M., Cushman, R. A., Cupp, A. S., \& Wood, J. R. (2014). Altered theca and cumulus oocyte complex gene expression, follicular arrest and reduced fertility in cows with dominant follicle follicular fluid androgen excess. PloS one 9, e110683.

Tezze, C., Romanello, V., \& Sandri, M. (2019). FGF21 as Modulator of Metabolism in Health and Disease. Frontiers in physiology 10, 419.

Thaker, M., \& Bilkei, G. (2005). Lactation weight loss influences subsequent reproductive performance of sows. Animal reproduction science, 88(3), 309-318.

Tuchscherer, M., Puppe, B., Tuchscherer, A., \& Tiemann, U. (2000). Early identification of neonates at risk: Traits of newborn piglets with respect to survival. Theriogenology 54(3), 371-388.

Van den Brand, H., Dieleman, S., Soede, N., \& Kemp, B. (2000). Dietary energy source at two feeding levels during lactation of primiparous sows: I. Effects on glucose, insulin, and luteinizing hormone and on follicle development, weaning-to-estrus interval, and ovulation rate. Journal of animal science, 78(2), 396-404.

Van der Ven, H. H., Al-Hasani, S., Diedrich, K., Hamerich, U., Lehmann, F., \& Krebs, D. (1985). Polyspermy in in vitro fertilization of human oocytes: frequency and possible causes. Annals of the new york academy of sciences, 442(1), 88-95.

Vinsky, M., Novak, S., Dixon, W., Dyck, M., \& Foxcroft, G. (2006). Nutritional restriction in lactating primiparous sows selectively affects female embryo survival and overall litter development. Reproduction, fertility and development, 18(3), 347-355.

Vural, F., Vural, B., Doğer, E., Çakıroğlu, Y., \& Çekmen, M. (2016). Perifollicular blood flow and its relationship with endometrial vascularity, follicular fluid EG-VEGF, IGF-1, and inhibin-a levels and IVF outcomes. Journal of assisted reproduction and genetics, 33(10), 1355-1362.

Walters, K.A., Eid, S., Edwards, M.C., Thuis-Watson, R., Desai, R., Bowman, M., Marren, A.J., \& Handelsman, D.J. (2019). Steroid profiles by liquid chromatography-mass spectrometry of matched serum and single dominant ovarian follicular fluid from women undergoing IVF. Reproductive biomedicine online 38(1), 30-37.

Wang, Q., \& Sun, Q.-Y. (2006). Evaluation of oocyte quality: morphological, cellular and molecular predictors. Reproduction, fertility and development, 19(1), 1-12.

Westhof, G., Westhof, K. F., Braendle, W. L., \& diZerega, G. S. (1991). Differential steroid secretion and gonadotropin response by individual tertiary porcine follicles in vitro. Possible physiologic role of atretic follicles. Biology of reproduction, 44(3), 461-468.

Whittemore, C., \& Morgan, C. (1990). Model components for the determination of energy and protein requirements for breeding sows: a review. Livestock production science, 26(1), 1-37.

Wientjes, J., Soede, N.M., Van der Peet-Schwering, C., Van Den Brand, H., \& Kemp, B. (2012a). Piglet uniformity and mortality in large organic litters: Effects of parity and pre-mating diet composition. Livestock science, 144(3), 218-229.

Wientjes, J., Soede, N., Aarsse, F., Laurenssen, B., Koopmanschap, R., Van Den Brand, H., \& Kemp, B. (2012b). Effects of dietary carbohydrate sources on plasma glucose, insulin and IGF-I levels in multiparous sows. Journal of animal physiology and animal nutrition 96(3), 494-505. 
Wientjes, J., Soede, N. M., Knol, E., Van den Brand, H., \& Kemp, B. (2013). Piglet birth weight and litter uniformity: Effects of weaning-to-pregnancy interval and body condition changes in sows of different parities and crossbred lines. Journal of animal science, 91(5), 2099-2107.

Wolfe, A., Divall, S., \& Wu, S. (2014). The regulation of reproductive neuroendocrine function by insulin and insulin-like growth factor-1 (IGF-1). Frontiers in neuroendocrinology, 35(4), 558-572.

Wolter, B., \& Ellis, M. (2001). The effects of weaning weight and rate of growth immediately after weaning on subsequent pig growth performance and carcass characteristics. Canadian journal of animal science, 81(3), 363-369.

Xia, P., Tekpetey, F. R., \& Armstrong, D. T. (1994). Effect of IGF-I on pig oocyte maturation, fertilization, and early embryonic development in vitro, and on granulosa and cumulus cell biosynthetic activity. Molecular reproduction and development, 38(4), 373-379.

Xie, S., Broermann, D., Nephew, K., Bishop, M., \& Pope, W. (1990). Relationship between oocyte maturation and fertilization on zygotic diversity in swine. Journal of animal science 68(7), 20272033.

Xue, L., Piao, X., Li, D., Li, P., Zhang, R., Kim, S. W., \& Dong, B. (2012). The effect of the ratio of standardized ileal digestible lysine to metabolizable energy on growth performance, blood metabolites and hormones of lactating sows. Journal of animal science and biotechnology, 3(1), 11.

Yang, H., Foxcroft, G., Pettigrew, J., Johnston, L., Shurson, G., Costa, A., \& Zak, L. (2000). Impact of dietary lysine intake during lactation on follicular development and oocyte maturation after weaning in primiparous sows. Journal of animal science, 78(4), 993-1000.

Yang, Y. X., Heo, S., Jin, Z., Yun, J. H., Choi, J. Y., Yoon, S. Y., Park, B. K., Yang, B. J. \& Chae, B. J. (2009). Effects of lysine intake during late gestation and lactation on blood metabolites, hormones, milk composition and reproductive performance in primiparous and multiparous sows. Animal reproduction science, 112(3), 199-214.

Yuan, B., Liang, S., Jin, Y.-X., Kwon, J.-W., Zhang, J.-B., \& Kim, N.-H. (2016). Progesterone influences cytoplasmic maturation in porcine oocytes developing in vitro. PeerJ, 4, e2454.

Yuan, Y., Van Soom, A., Leroy, J., Dewulf, J., Van Zeveren, A., de Kruif, A., \& Peelman, L. (2005). Apoptosis in cumulus cells, but not in oocytes, may influence bovine embryonic developmental competence. Theriogenology, 63(8), 2147-2163.

Zak, L., Xu, X., Hardin, R., \& Foxcroft, G. (1997). Impact of different patterns of feed intake during lactation in the primiparous sow on follicular development and oocyte maturation. Journal of reproduction and fertility, 110(1), 99-106.

Zak, L., Williams, I., Foxcroft, G., Pluske, J., Cegielski, A., Clowes, E., \& Aherne, F. (1998). Feeding lactating primiparous sows to establish three divergent metabolic states: I. Associated endocrine changes and postweaning reproductive performance. Journal of animal science, 76(4), 1145-1153.

Zhong, H., Wang, P., Song, Y., Zhang, X., Che, L., Feng, B., Lin, Y., Xu, S., Li, J., \& Wu, D. (2018). Mammary cell proliferation and catabolism of adipose tissues in nutrition-restricted lactating sows were associated with extracellular high glutamate levels. Journal of animal science and biotechnology 9, e78. 
Summary 

Modern sow lines have been genetically selected for increased litter size. This increase in litter size, however, is also associated with higher piglet mortality. This piglet mortality can be partly explained by lower piglet birth weights and higher within-litter variation in birth weight. The main hypothesis of this thesis is that these factors, the lower piglet birth weights and the higher within-litter variation, are (at least partly) explained by impaired follicular development of sows during and after lactation, as this is the period in which antral follicles develop that contain the oocytes that will give rise to the next litter. Increased litter size also results in increased metabolic demands of modern sows during lactation, due to the necessary higher milk production. As the metabolic state highly influences follicular development, the increased metabolic demands may therefore impair follicular development and contribute to lower piglet birth weights and higher within-litter variation in piglet birth weight. It is not clear, however, which metabolic processes play the most significant roles in influencing follicular developmental competence in modern sows.

Increased understanding of the origin of lower piglet birth weights in modern sows may be achieved by identification of molecular physiological characteristics which determine follicular developmental competence and how these are influenced by metabolism. Therefore, the aims of this thesis were: 1) To establish physiological and molecular characteristics of follicular developmental competence in sows and 2) To better understand physiological relations between the metabolic state of sows and follicular developmental competence.

This thesis describes results from two pig studies. In the first study, follicular development and oocyte quality were assessed in multiparous sows at the onset of the follicular phase (at weaning), while in the second study, follicular development and oocyte quality were assessed in primiparous sows at the mid-follicular phase (48 after weaning). In both studies, the metabolic state of the sows was assessed to investigate physiological relations between metabolism during lactation and follicular developmental competence. In the first study, multiparous sows were all full-fed during lactation, while in the second study, half of the primiparous sows were restricted-fed during the last two weeks of lactation.

In Chapter $\mathbf{2}$ of this thesis, I aimed to identify possible follicular causes of piglet birth weight variation by studying follicular development of multiparous sows at weaning in sows that differ in their estimated breeding value (EBV) for piglet birth weight variation (Study 1). In addition, relations between the metabolic state of these sows during lactation and follicular development were assessed. No significant relations between EBV for birth weight variation and any of the measured follicular or metabolic parameters were found. We did, however, find relations between metabolic losses and subsequent follicle development and -quality. For example, average follicle size of the 15 largest follicles (the presumptive ovulatory follicle pool) was negatively related to serum creatinine levels, a marker for protein breakdown, and this follicle size was surprisingly, positively related to a higher backfat depth loss during lactation. As sows with a high backfat loss also had higher backfat levels at the start of lactation, it was hypothesised that modern sows with more backfat at the start of lactation 
are able to mobilise more energy from backfat during lactation to support follicular development thereby sparing protein reserves. To conclude, although unfortunately no relations between EBV and follicular development have been found, our study does show that energy mobilization from different sources during lactation, adipose tissues or muscle reflecting fat or lean mass, respectively, could have divergent effects on follicular development at weaning.

Identification of molecular physiological characteristics which determine follicular developmental competence, may provide new insights in the origin of lower piglet birth weights in sows. Therefore, follicular granulosa cell gene and protein expression and follicular fluid composition were assessed in relation to two follicular competence markers, average follicle size (Chapter 3) and cumulus-oocyte complex (COC) morphology (Chapter 4 ) in the sows of Study 1.

In Chapter 3, I investigated granulosa cell processes in large and small follicles of the pool of 15 largest follicles (the presumptive ovulatory follicle pool). Granulosa cells of smaller antral follicles showed increased cell proliferation, which was accompanied by a metabolic shift towards aerobic glycolysis, similar to other highly proliferating cells. High granulosa cell proliferation rates in smaller follicles may be regulated via increased expression of receptors for locally produced mitogens, such as androgen receptor (AR) and epidermal growth factor receptor (EGFR). While granulosa cells of smaller follicles in the pool were more proliferative, granulosa cells of larger follicles expressed more maturation markers, such as insulin-like growth factor 1 (IGF1) and angiopoietin 1. Thus, in this chapter, granulosa cell processes and key genes were identified which may determine antral follicular developmental competence at the onset of the follicular phase.

In Chapter 4, I studied follicular developmental competence, by studying relations between follicular fluid steroid profiles and oocyte health of the presumptive ovulatory follicle pool. Sows with a high compared to a low percentage healthy COCs ( $<70 \%$ vs. $\geq 70 \%$ healthy), had higher $17 \beta$-estradiol, 19-norandrostenedione, progesterone and $\alpha$-testosterone levels, while cortisol levels were lower. In addition, a larger average follicle size of the 15 largest follicles was related to higher $17 \beta$-estradiol levels. Transcriptome and selective protein analysis of granulosa cells of healthy follicles of sows with a high percentage healthy COCs showed increased expression of genes involved in steroidogenesis and follicular maturation, and a differential expression of genes regulating granulosa cell apoptosis. Also the metabolic state of the sows affected the level of $\mathrm{COC}$ health, as sows with a high percentage healthy COCs lost less weight during lactation and had higher serum IGF1 levels at weaning. The level of COC health at the onset of the follicular phase was thus highly related to follicular steroidogenesis.

Both follicle size and percentage healthy COCs, two follicular competence markers, were found to be related to follicular fluid steroid levels. If relations between follicular and serum steroid levels could be established, serum steroid profiles may be used to monitor follicular 
development. This was investigated in Chapter 5, using samples of Study 1 and 2. I identified serum steroids that reflect follicular development in the early stages of the follicular phase and established whether follicular fluid steroid levels correspond to those in serum. Serum steroid levels were never related to average follicle size of the pool. Moreover, no difference in serum steroid levels was observed when levels at the onset of the follicular phase were compared to levels in the mid-follicular phase. Serum steroid levels therefore poorly reflect the developmental stage of the follicle pool in the first half of the follicular phase of the estrous cycle and can therefore unfortunately not be used to monitor follicular development.

Another way to identify possible follicular causes of piglet birth weight and within-litter variation in birth weight, is by reducing lactational feed intake in sows. A low lactational feed intake increases mobilization of energy from body tissues which has been found to negatively influence piglet birth weight and variation in piglet birth weight of the next litter. Studying effects of lactational feed restriction on follicular and oocyte developmental competence, may therefore elucidate follicular characteristics that finally influence piglet birth weight and variation in piglet birth weight. Chapter 6 describes effects of feed restriction during the last two weeks of a 24-day lactation on follicular developmental competence at the mid-follicular phase, in primiparous sows (Study 2). Feed restriction impaired follicular developmental competence, as shown by a smaller average size of the 15 largest follicles and reduced cumulus-oocyte complex expansion after $22 \mathrm{~h}$ in vitro maturation. Feed restriction also impaired oocyte quality, which was shown by a delayed zygote development $24 \mathrm{~h}$ after in vitro fertilization and a higher incidence of polyspermy. This reduced follicular and oocyte developmental competence may be influenced by the lower IGF1 and steroid levels detected in the follicular fluid of restricted-fed sows. Together, these results implicate that lactational feed restriction impairs follicular steroid and growth factor production which reduces oocyte developmental competence. These impairments may at least partly explain lower piglet birth weights.

Lactational feed intake does not only affect sow performance, but also affects piglet performance by changing sow milk production, as described in Chapter 7 (Study 2). A higher feed intake during lactation resulted in a higher milk fat percentage at weaning and a higher total milk fat and protein production in the last week of lactation. Both a better body condition (higher body weight, loin muscle depth and backfat depth) at parturition and more body tissue mobilization (backfat depth loss and loin muscle depth loss) were related to a higher milk fat and/or protein production in the last week of lactation. Together, findings from Chapter $\mathbf{6}$ and 7 suggest that a higher lactational feed intake benefits both sow performance, by supporting follicular development during lactation, as well as piglet performance, by increasing sow milk production. However, more tissue mobilization during lactation impairs follicular developmental competence while it may be beneficial for milk production which supports litter weight gain. It therefore remains a challenge to design optimal feeding strategies that benefit both the current litter (sustained milk production) as well as the next litter (supporting follicular development). 
In conclusion, this thesis shows that follicular developmental competence of the presumptive ovulatory follicle pool is associated with more granulosa cell differentiation and less proliferation, more steroidogenesis and a higher IGF1 production. In Chapter 8, it is argued that these identified processes may also be important for subsequent embryo and foetal survival and development, and finally, piglet birth weight and survival. The metabolic state of modern sows during lactation highly influences follicular developmental competence, already from the first half of the follicular phase onward. In addition, in primiparous modern sows, lactational weight loss influenced litter characteristics of the next litter. This provides further evidence for the hypothesis of this thesis that impairments in follicular developmental competence as established during lactation may at least partially explain the reduced reproductive performance in the next cycle and lower piglet birth weights. The degree and type of body tissue mobilization i.e. adipose tissue or lean mass, highly influences follicular development during lactation as well as milk production and composition, which are both important factors for sow performance. It seems that especially mobilization of energy from lean mass in sows with low adipose tissue reserves, negatively impacts follicular developmental competence, while mobilization of protein from lean mass may be beneficial for milk protein production to support piglet growth. It therefore remains a challenge to optimize current sow management strategies benefit sow- and piglet performance. 


\section{Acknowledgements}



At the end of my thesis I would like to use the opportunity to thank everyone who made this thesis possible.

First of all, I would like to thank my (co)promotors: Jaap, Bas, Nicoline and Katja. Jaap, I greatly value and appreciate all your novel and creative ideas which always gave me new energy. I also really appreciate that you always took the time to provide honest and direct feedback which helped me grow as a scientist. Bas, thank you for your continuous enthusiasm and optimism. You always had valuable suggestions for additional calculations and analyses. Nicoline and Katja, together you formed a great supervision team. Not only because your scientific expertise complements each other so well, but also because you have supervised me both in your own unique way. Nicoline, I really appreciate that you always took the time to provide elaborate feedback. Your attention for detail and your critical questions have helped to keep me sharp throughout my PhD. Katja, thank you for always being available to answer my questions or give me advice. I really appreciate that you have given me so much trust and freedom to develop myself into an independent and confident scientist.

Next, I would like to acknowledge several parties with whom I collaborated and who made this research possible. First of all, I would like to thank Marco Blokland and Frederike van Tricht of Wageningen Food Safety Research for their willingness to participate in this research. Also many thanks to Shohreh Kesthkar of the Nutrition, Metabolism and Genomics Group, for her time and patience in helping me with the laser capture microdissection. I would also like to thank Bernard Roelen, Leni van Tol and Eric Schoevers, of the faculty of Veterinary Medicine of Utrecht University. I really am grateful that you were willing to teach me in vitro embryo production and spend a lot of personal time (including weekends) to help me execute the measurements. I also want to acknowledge the financial and in-kind contribution of Topigs Norsvin. In particular, I would like to thank Louisa Zak and Egbert Knol, for bringing a different perspective into my research project and for helping me to reflect on my data in a different way.

Furthermore, I would like to thank my colleagues at ADP and HAP. I specifically would like to thank the technicians who provided a lot of technical and social support during my PhD project. Without your help I would have never been able to finish my research in time. Melissa and Inge, thanks for your positivity and always being available to help. Annelies, my go-to person with any kind of question or problem, together we faced quite some challenges trying to make existing protocols work in pigs (but we did it!). Hans and Marcel, thank you for helping me optimize all the histology protocols and Rudie, many thanks for all the analyses and your enthusiasm to take up the challenge to make the measurements work in follicular fluid and milk. Henk and Anja, thank your for all of your help and organising the histology lab.

My gratitude also goes to Evert, for helping me with the microarray data analysis, Klaas for his help with some of the statistical analyses, and Annet, Annika, Corine, Irene, Nanette and Lora 
of the secretariat for their practical support. Bjorge, Anouschka, Carol, Manon and also Arjan and Rinie of CARUS, thank you for all your help with the execution of the animal experiment(s) and making the long working days an enjoyable and unforgettable experience.

I would like to thank all students and guest researchers who were (in)directly working on my PhD project. In particular, I would like to thank Maud, Quirijn, Vera, Laura and Yu who were directly involved in my project.

Of course, I also really want to thank my HAPpy PhD colleagues: Lianne, Taolin, Bart, Anna, Joëlle, José, Jeske, Miranda, Wenbiao, Li, Marjanne, Maria, Lonneke, lyabo and Jing. I feel very lucky to have been able to share this experience with you guys. Thank you for all for your support and the necessary distractions in the form of dinners, coffee breaks, PhD parties, filming the movies and the coffee or lunch walks. Despite all of your hard work, you were always available to help. I would consider myself very lucky if I would find such great colleagues again.

Special thanks to my paranymphs Lianne and Taolin. I really appreciate that you are willing to take the time to help me with my defence. Lianne, thanks for all of your help and advice during the last 4 years, and of course for your motivating Sinterklaas poems. Taolin, I still hope to see you in San Francisco after your PhD :). I am very happy to have you as my paranymphs.

We come to the end of this thesis which marks the end of a four-year journey. To finish, I want to take this opportunity to thank my parents, family, friends and partner. I would really like to thank you for continous support. 
About the author 



\section{Curriculum Vitae}

Natasja Geertruda Johanna Costermans was born on the $8^{\text {th }}$ of November 1990. She started her BSc in Animal Sciences at Wageningen University in 2010 and received her cum laude Bachelor degree in 2013. In the same year, she started her MSc program Animal Sciences, also at Wageningen University, with the specialisation Animal Health and Behaviour. During this MSc, she did a minor thesis at Linköping University in Sweden, where she studied effects of hypoxia during incubation on intestinal development in chicken. She did her major thesis at Adaptation Physiology, Wageningen University, where she studied carbohydrate recognition of natural antibodies. During her MSc, Natasja participated in the research master cluster, where she wrote a PhD proposal and participated in the NWO-WIAS graduate programme competition in 2015. She was the winner of this competition, which resulted in funding for her PhD project. She obtained her cum laude MSc degree in June 2015. In September 2015, Natasja started with her PhD research entitled: "Physiological and molecular aspects of ovarian follicular developmental competence in sows". This PhD project was a collaboration between Human and Animal Physiology and Adaptation Physiology, at Wageningen University. During her PhD, she participated in the educational program of the Graduate School WIAS. She was also involved in teaching activities of both chair groups and supervised BSc and MSc students. Currently, she is working as a postdoc at the University of California in San Francisco (UCSF) where she studies potential causes of age-related decline in oocyte quality. 


\section{List of publications}

\section{Peer-reviewed publications}

F. R.C. K. Almeida*, N. G. J. Costermans*, N. M. Soede, A. Bunschoten, J. Keijer, B. Kemp, K. J. Teerds. (2018). Presence of anti-Müllerian hormone (AMH) during follicular development in the porcine ovary. PLOS ONE, 13(7):e0197894. DOI:10.1371/journal.pone.0197894. *Contributed equally to this work.

N. G. J. Costermans, K. J. Teerds, J. Keijer, E. F. Knol, R. E. Koopmanschap, B. Kemp, N. M. Soede. (2019). Follicular development of sows at weaning in relation to estimated breeding value for within-litter variation in piglet birth weight. Animal 13(3), 554-563. DOI:10.1017/S1751731118001684.

N. G. J. Costermans, J. Keijer, B. Kemp, E. M. van Schothorst, S. Keshtkar, A. Bunschoten, N. M. Soede and K. J. Teerds. (2019). In ovaries with high or low variation in follicle size, granulosa cells of antral follicles exhibit distinct size-related processes. Molecular Human Reproduction, 25(10), 614-625. DOI:10.1093/molehr/gaz042.

N. G. J. Costermans, K. J. Teerds, A. Middelkoop, B. A. Roelen, E. J. Schoevers, H. T. A. van Tol, B. F. A. Laurenssen, R. E. Koopmanschap, Y. Zhao, M. Blokland, F. van Tricht, L. J. Zak, J. Keijer, B. Kemp and N. $M$. Soede (2019). Consequences of negative energy balance on follicular development and oocyte quality in primiparous sows. Biology of Reproduction, DOI:10.1093/biolre/ioz175.

A. Middelkoop, N. G. J. Costermans, B. Kemp, J. E. Bolhuis. (2019). Feed intake of the sow and playful creep feeding of piglets influence piglet behaviour and performance before and after weaning. Scientific Reports, DOI:10.1038/s41598-019-52530-w.

N. G. J. Costermans, N. M. Soede, F. van Tricht, M. Blokland, B. Kemp, J. Keijer and K. J. Teerds. Follicular fluid steroid profile in sows: relationship to follicle size and oocyte quality. Biology of Reproduction, DOI:10.1093/biolre/ioz217.

N. G. J. Costermans, N. M. Soede, M. Blokland, F. van Tricht, J. Keijer, B. Kemp and K. J. Teerds. Steroid profile of porcine follicular fluid and blood serum: relation with follicular development. Physioloigcal Reports, DOI:10.14814/PHY2.14320.

\section{Expected publications}

N. G. J. Costermans, N. M. Soede, A. Middelkoop, B. F. A. Laurenssen, R. E. Koopmanschap, L. J. Zak, E. F. Knol, J. Keijer, K. J. Teerds and B. Kemp. Influence of the metabolic state during lactation on milk production in modern sows. Manuscript submitted.

V. Coleman, L. Meng, N. G. J. Costermans, Y. Zhao, M. Ost, A. Voigt, A. Bunschoten, J. Keijer, K. J. Teerds and $\mathrm{S}$. Klaus. Skeletal muscle pseudo-starvation driven energy expenditure negatively affects ovarian follicular development in mice. Manuscript submitted. 


\section{Education and Training}

The Basic Package - 1.8 ECTS

WIAS Introduction Day

Course on philosophy of science and/or ethics

Disciplinary Competences - 13.1 ECTS

Writing of a literature review

Design of Experiments

Design of breeding programs with Genomic Selection

Multiscale Imaging for the Study of Animal Reproduction (INRA, Tours, France)

SSR conference 2018 pre-meeting ovarian workshop (New Orleans, LA, USA)

HAP journal clubs

Labvisit at the Center for Reproductive Sciences, UCSF (San Francisco, CA, USA)

\section{Professional Competences - 6.3 ECTS}

Effective behaviour in your professional surroundings

Interpersonal communication for PhD students

Posters and pitching

Teaching and supervising thesis students

The essentials for writing and presenting a scientific paper

Reviewing a scientific paper

Career perspectives

\section{Presentation Skills - 4 ECTS}

WIAS Science Day 2016 'poster presentation' (Wageningen, The Netherlands)

ICPR 2017 'poster presentation' (Missouri, MA, USA)

SSR conference 2018 'poster presentation' (New Orleans, LA, USA)

WIAS science day 2019 'oral presentation' (Wageningen, The Netherlands)

SSR conference 2019 'poster presentation' (San Jose, CA, USA)

\section{Teaching competences - 6 ECTS}

Supervision practical Human and Animal Biology

Supervision practical Nutritional aspects of food

Supervision practicals Behavioural endocrinology

Supervision practicals Reproduction and Fertility

Supervision practical Integrated Human Physiology

Supervising $7 \mathrm{MSc}$ and $3 \mathrm{BSc}$ thesis students 


\section{Colophon}

The Netherlands Organisation for Scientific Research (NWO) and Wageningen Institute for Animal Sciences (WIAS) are acknowledged for providing the NWO-WIAS graduate programme 2015 grant for financial contribution to the author of this thesis. This work is supported by Topigs Norsvin Research Centre B. V., The Netherlands. Financial support from Human and Animal Physiology, Adaptation Physiology Group and Topigs Norsvin for printing this thesis is gratefully acknowledged.

Layout by: Natasja Costermans

Cover design by: Hanneke Wetzer

Printed by: ProefschrifMaken | DigiForce 

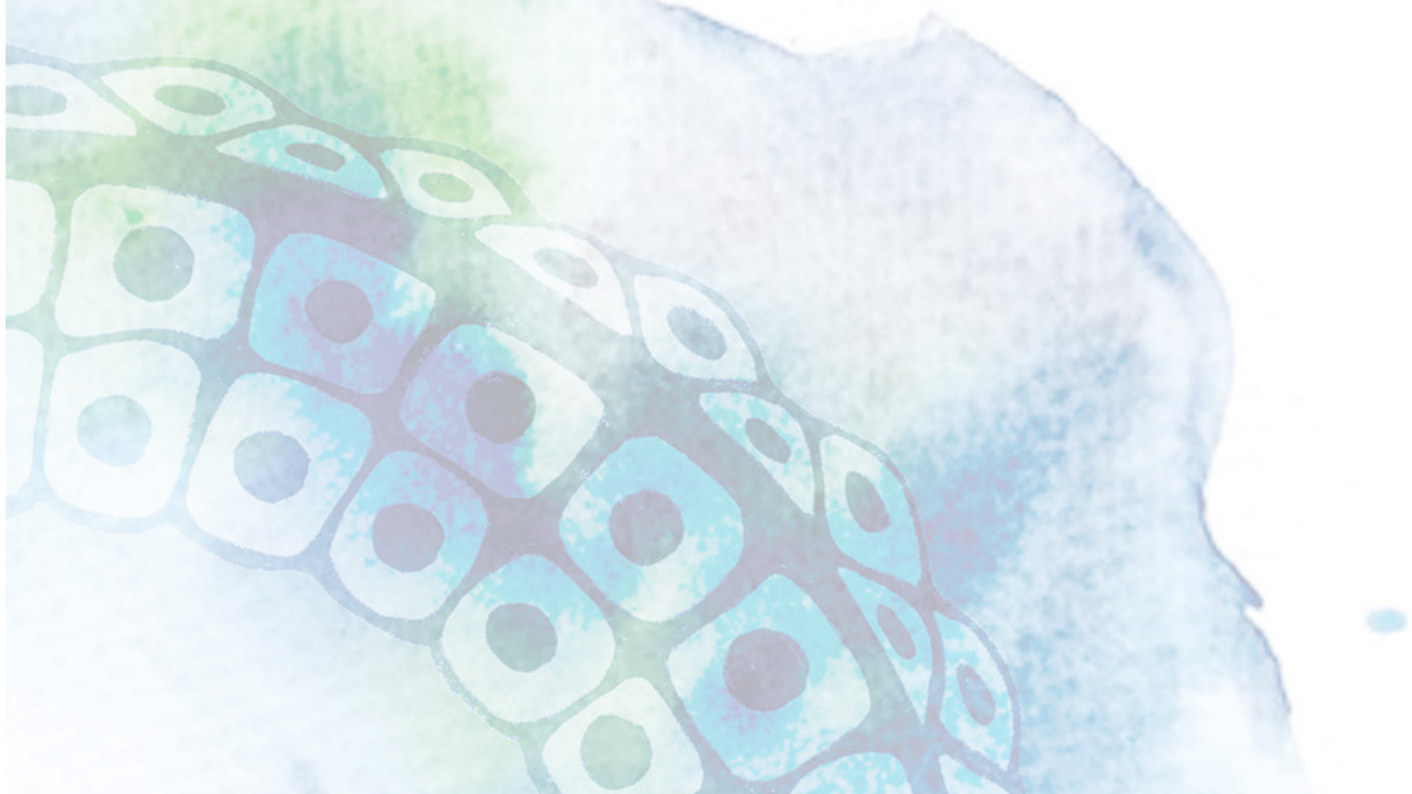